\title{
Beeinflussung des Geräuschs von Induktionsmaschinen durch innovative Ständer- und Läufergeometrien
}

\author{
Von der Fakultät für Elektrotechnik und Informatik \\ der Gottfried Wilhelm Leibniz Universität Hannover \\ zur Erlangung des akademischen Grades \\ Doktor-Ingenieur \\ (abgekürzt: Dr.-Ing.) \\ genehmigte Dissertation \\ von
}

Dipl.-Ing. Eike Matthias Garbe

geboren am 12.11.1982 in Hannover

2015 
1. Referent: Prof. Dr.-Ing. Bernd Ponick

2. Referent: Prof. Dr.-Ing. Ekkehard Bolte

Tag der Promotion: 15.06.2015 


\section{Vorwort}

Die vorliegende Arbeit entstand wärend meiner dreijährigen Tätigkeit als wissenschaftlicher Mitarbeiter am Institut für Antriebssysteme und Leistungselekronik (IAL) der Leibniz Universität Hannover sowie parallel zu meiner anschließenden Industrietätigkeit.

Ich danke Herrn Prof. Dr.-Ing. Bernd Ponick, der mich zu dieser Arbeit ermuntert hat und immer gerne zu fachlichen Diskussionen bereit war. Insbesondere danke ich ihm dafür, mir zwei je dreieinhalbmonatige Aufenthalte als Gastwissenschaftler an Norges teknisknaturvitenskapelige Unversitet (NTNU, Trondheim, Norwegen) und an der Kungliga Tekniska högskolan (KTH, Stockholm, Schweden) zu ermöglichen. Mein Dank gilt dabei auch den Mitarbeitern am Institut für „elkraftteknikk“ an der NTNU sowie der Abteilung für ,elektrisk energiomvandling“" an der KTH für die jeweils entgegengebrachte Gastfreundschaft.

Des Weiteren danke ich Herrn Prof. Dr.-Ing. Ekkehard Bolte für die Übernahme des Koreferates sowie Herrn Prof. Dr.-Ing. Axel Mertens für die Übernahme des Vorsitzes der Prüfungskommision.

Meinen Kollegen am IAL danke ich für die stets angenehme Zusammenarbeit.

Ein besonderer Dank gilt meiner Familie. Hier vor allem meinen Eltern, die mich während meiner gesamten Ausbildung immer unterstützt haben. Insbesondere gilt der Dank jedoch meiner Frau und meinen Söhnen, die mich stets zum Erreichen meiner Ziele motiviert haben.

Ålesund im Juni 2015

Eike Garbe 
Für Torbjørn 


\section{Inhaltsverzeichnis}

Verzeichnis der wichtigsten Formelzeichen und Symbole IX

Kurzfassung $\quad$ XI

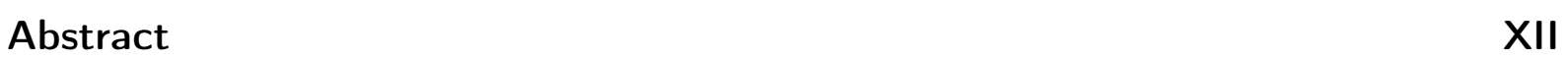

$\begin{array}{lll}1 & \text { Einleitung } & 1\end{array}$

2 Grundlagen 4

2.1 Koordinatensvstem . . . . . . . . . . . . . . . . . . . . . . . . . . . .

2.2 Phvsiologische Grundlagen des Hörens . . . . . . . . . . . . . . . . . . 4

2.3 Schrägung . . . . . . . . . . . . . . . . . . . 5

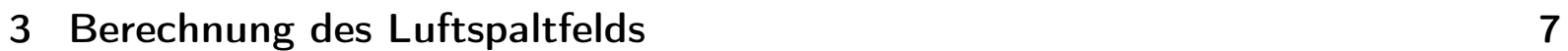

3.1 Strombelagswellen der Ständerwicklung . . . . . . . . . . . . . . . . . . . . 7

3.2 Wicklungsfaktor der Ständerwicklung . . . . . . . . . . . . . . . . . . . 11

3.3 Felderregerwellen der Ständerwicklung . . . . . . . . . . . . . . . . . 11

3.4 Luftspaltleitwertwellen . . . . . . . . . . . . . . . . . . . . . . . . . . . 12

3.4 .1 Schrägung . . . . . . . . . . . . . . . . . . . 16

3.4 .2 Magnetische Spannung über den Eisenwegen . . . . . . . . . . . . . 17

3.5 Ständerseitig erregte Induktionswellen f . . . . . . . . . . . . . . . 18

3.6 Läuferrückwirkung . . . . . . . . . . . . . . . . . . . . . . . . . . . . . . . 19

3.6 .1 Induzierte Spannung . . . . . . . . . . . . . . . . . 20

3.6 .2 Nutstreuinduktivitäten . . . . . . . . . . . . . . . . . . . . . . . 24

3.6 .3 Einfluss der Stromverdrängung . . . . . . . . . . . . . . . . . . 25

3.6.4 Diskrete Betrachtung des Läuferkäfigs. . . . . . . . . . . . . . 30

3.6.5 Kontinuierliche Betrachtung des Läuferkäfigs . . . . . . . . . . . . . 33

3.6.6 Wicklungsfaktor der Läuferwicklung . . . . . . . . . . . . . . . . . . 39

3.6.7 Schrägung . . . . . . . . . . . . . . . . . . . . . . . . . . . 41

3.6 .8 Rückwirkung auf den Ständerstrom . . . . . . . . . . . . . . . . . . 41

4 Geräuschanregung und Drehmomentbildung 42

4.1 Drehmoment . . . . . . . . . . . . . . . . . . . . . . . 42

4.1 .1 Schrägung . . . . . . . . . . . . . . . . . . . 46

4.1.2 Drehmoment des kontinuierlichen Modells . . . . . . . . . . . . 46

4.2 Radialkraftwellen . . . . . . . . . . . . . . . . . . . . 47

4.3 Tangentialkräfte . . . . . . . . . . . . . . . . . . . . . . . . . . 48

4.4 Jochzugwellen . . . . . . . . . . . . . . . . . . . . . . . . . . . 54

4.5 Schwingung des Jochs und Schallabstrahlung . . . . . . . . . . . . . . 56

4.6 Schlussfolgerung . . . . . . . . . . . . . . . . . . . . . . . . . . . . . . . . . . . 59 
5 Untersuchung des Berechnungsverfahrens 60

5.1 Vereinfachte Berechnung der resultierenden Luftspaltleitwertwellen . . . . . 60

5.2 Vergleich von diskreter und kontinuierlicher Betrachtung des Läufers . . . . 61

5.3 Methoden zur Drehmomentberechnung . . . . . . . . . . . . . . . 65

5.4 Flussaufteilung zur Berechnung der Tangentialkräfte an den Zähnen . . . . 66

5.5 Berechnung der Tangentialkräfte an den Zähnen . . . . . . . . . . . . . 67

6 Variation der Ständergeometrie 69

6.1 Gleichmäßige Ständergeometrie . . . . . . . . . . . . . . . . . . 69

6.2 Variation der Zahnbreiten $\ldots \ldots \ldots \ldots \ldots \ldots$. . . . . . . . . . . . . .

6.3 Variation der Luftspaltweite $\ldots \ldots \ldots \ldots \ldots \ldots \ldots$

6.4 Phasenwinkelkorrektur ... . . . . . . . . . . . . . . . 77

6.4.1 Einfluss auf die Variation der Luftspaltweite . . . . . . . . . . 78

6.4 .2 Nachteile der Phasenwinkelkorrektur . . . . . . . . . . . . 80

\begin{tabular}{lll}
\hline & Entwurf und Untersuchung von Beispielmaschinen & 81
\end{tabular}

7.1 Entwurfsgang . . . . . . . . . . . . . . . . . . . . . 81

7.2 Gleichmäßige Geometrien . . . . . . . . . . . . . . . . . . 82

7.3 Variation der Läufergeometrie . . . . . . . . . . . . . . . . . . 83

7.3 .1 Wahl der Läufernutbreiten . . . . . . . . . . . . . . . . . 83

7.3 .2 Einbeziehung von Nichtlinearitäten . . . . . . . . . . . . . . 85

7.3 .3 Maschine .B. . . . . . . . . . . . . . . . . . . . 86

7.3 .4 Maschine .C. . . . . . . . . . . . . . . . . . . . 88

7.4 Variation der Ständergeometrie . . . . . . . . . . . . . . . . . . . 101

7.4 .1 Maschine .A. . . . . . . . . . . . . . . . . . . . . . 101

7.4 .2 Maschine .C. . . . . . . . . . . . . . . . . . . . . 104

7.4.3 Reluktanzmaschine auf Basis von Maschine ..C. . . . . . . . . 111

8 Zusammenfassung 115 


\section{Symbole}

$\begin{array}{ll}x & \text { Allgemeine Variable } \\ x & \text { Augenblickswert einer } \\ & \text { zeitveränderlichen Größe } \\ \mathrm{x} & \text { Konstante } \\ \hat{x} & \text { Amplitudenwert } \\ \underline{x} & \text { Komplexe Variable } \\ \Re\{\underline{x}\} & \text { Realteil einer komplexen } \\ & \text { Variable } \\ \Im\{\underline{x}\} & \text { Imaginärteil einer komplexen } \\ & \text { Variable } \\ \underline{\vec{x}} & \text { Komplexer Raumzeiger } \\ X & \text { Allgemeine Variable }\end{array}$

\section{Formelzeichen}

$\begin{array}{llll}A & \text { Fläche } & k_{\mathrm{l}} & \text { Stromverdrängungskoeffizient } \\ A, a & \text { Strombelag } & & \text { der Stabstreuinduktivität } \\ a & \text { Anzahl paralleler } & L & \text { Induktivität } \\ & \text { Wicklungszweige } & l_{\mathrm{i}} & \text { ideelle Blechpaketlänge } \\ a & \text { Wichtungsfaktor } & M & \text { Drehmoment } \\ B & \text { Induktion } & M & \text { Gegeninduktivität } \\ b & \text { Wichtungsfaktor } & m & \text { Anzahl der Stränge } \\ b_{2} & \text { Periodenanzahl der } & N_{1} & \text { Anzahl der Ständernuten } \\ & \text { Läufernutmodulation } & N_{2} & \text { Anzahl der Läufernuten } \\ b_{\mathrm{N}} & \text { Nutbreite im Bogenmaß } & \mathbb{N} & \text { Menge der nicht-negativen } \\ b_{\mathrm{N}}^{*} & \text { Nutbreite im Längenmaß } & & \text { ganzen Zahlen } \\ b_{\mathrm{No}} & \text { Breite des Nutschlitzes } & \mathbb{N} * & \text { Menge der positiven ganzen } \\ b_{\mathrm{z}} & \text { Zahnbreite im Bogenmaß } & & \text { Zahlen (ohne Null) } \\ D & \text { Durchmesser } & n_{\nu} & \text { Anzahl betrachteter Feldwellen } \\ I, i & \text { Strom } & n_{\mathrm{q}} & \text { Nenner der Lochzahl } q \text { (gekürzt) } \\ \mathrm{j} & \text { imaginäre Einheit } & p & \text { Polpaarzahl } \\ \mathrm{e} & \text { Einheitsvektor } & q & \text { Lochzahl } \\ E & \text { Elektrische Feldstärke } & R & \text { Widerstand } \\ F & \text { Kraft } & r & \text { Radius } \\ F_{\mathrm{n}} & \text { Normalkraft } & S & \text { Elektrische Durchflutung } \\ F_{\mathrm{t}} & \text { Tangentialkraft } & s & \text { Schlupf } \\ f & \text { Frequenz } & t & \text { Zeit } \\ h_{\mathrm{No}} & \text { Höhe des Nutschlitzes } & W, u & \text { Spannung } \\ h_{\mathrm{z}} & \text { Zahnhöhe } & V & \text { Felderregung } \\ J_{n} & \text { Besselfunktion } & V & \text { Magnetische Spannung } \\ k_{\mathrm{r}} & \text { Stromverdrängungskoeffizient } & v & \text { Geschwindigkeit } \\ & \text { des Stabwiderstands } & W & \text { Energie } \\ & & w & \text { Spulenweite } \\ & & & \end{array}$

$X \quad$ Effektivwert einer harmonischen Größe

$\bar{x} \quad$ Mittelwert

$\mathbf{x} \quad$ Vektor

X Matrix

$\tilde{x} \quad$ Unterscheidungsmarke

$\breve{x} \quad$ Unterscheidungsmarke

$x^{\prime} \quad$ Bezogene Größe

$x^{\prime} \quad$ Unterscheidungsmarke

$x^{\prime \prime} \quad$ Unterscheidungsmarke

$x^{*} \quad$ Längenmaß (sonst Bogenmaß)

$\angle \quad$ Winkel

$=: \quad$ Rechtsseitige Definition $w \quad$ Spulenweite 


$\begin{array}{ll}w & \text { Windungszahl } \\ w_{N} & \text { Anzahl in Reihe geschalteter } \\ & \text { Leiter in einer Nut } \\ x & \text { Winkelkoordinate im Bogenmaß } \\ x^{*} & \text { Winkelkoordinate im } \\ & \text { Längenmaß } \\ x_{1} & \text { ständerfeste Winkelkoordinate } \\ x_{2} & \text { läuferfeste Winkelkoordinate } \\ x_{(n)} & \text { Position der Nut }(n) \\ \Delta x_{\mathrm{N}} & \text { Nutabstand } \\ x_{\mathrm{z}(n)} & \text { Position des Zahns ( } n) \\ \Delta x_{\mathrm{z}} & \text { Zahnabstand } \\ z & \text { Koordinate in Richtung der } \\ & \text { Längsachse } \\ \mathbb{Z} & \text { Menge der ganzen Zahlen } \\ \mathbb{Z}^{*} & \text { Menge der ganzen Zahlen ohne } \\ & \text { Null } \\ z_{\mathrm{q}} & \text { Zähler der Lochzahl } q \text { (gekürzt) } \\ \alpha & \text { Abplattungsfaktor } \\ \beta & \text { Faktor der Induktionsabsenkung } \\ & \text { über einer Nut } \\ \gamma_{\mathrm{schr}} & \text { Schrägungswinkel } \\ \delta & \text { Luftspaltweite } \\ \Phi & \text { magnetischer Fluss } \\ \vartheta & \text { Modulationsstärke } \\ \vartheta(r) & \text { Verteilungsfunktion der } \\ & \text { Induktion }\end{array}$

$\begin{array}{ll}\kappa & \text { elektrische Leitfähigkeit } \\ \kappa & \text { Polpaarzahl einer Drehwelle } \\ \Lambda & \text { magnetischer Leitwert } \\ \lambda & \text { magnetische Leitwertdichte } \\ \lambda & \text { Wellenlänge } \\ \mu & \text { Permeabilität } \\ \mu & \text { Polpaarzahl einer Drehwelle } \\ \mu_{0} & \text { Permeabilität im Vakuum } \\ \mu_{\mathrm{r}} & \text { relative Permeabilität } \\ \nu & \text { Polpaarzahl einer Drehwelle } \\ \xi & \text { Wicklungsfaktor } \\ \xi_{\mathrm{K}} & \text { Kopplungsfaktor mit einer } \\ & \text { Käfigmasche } \\ \xi_{\mathrm{N}} & \text { Nutschlitz- bzw. Breitenfaktor } \\ \xi_{\text {schr }} & \text { Schrägungsfaktor } \\ \xi_{\mathrm{u}} & \text { Einkopplungsfaktor } \\ \rho & \text { Drehwinkel des Läufers } \\ \rho_{0} & \text { Drehwinkel des Läufers zum } \\ & \text { Zeitpunkt } t=0 \\ \sigma & \text { Schrägungskoeffizient einer } \\ & \text { Drehwelle } \\ \tau_{\mathrm{p}} & \text { Polteilung } \\ \Psi & \text { magnetischer Verkettungsfluss } \\ \omega & \text { Kreisfrequenz } \\ \Omega & \text { Winkelgeschwindigkeit des } \\ & \text { Läufers }\end{array}$

\section{Indizes}

$\begin{array}{llll}1 & \text { Ständer } & \text { mech } & \text { mechanisch } \\ 2 & \text { Läufer } & \mathrm{N} & \text { Nut } \\ \mathrm{A} & \text { Strombelag } & \mathrm{n} & \text { Normalkomponente } \\ \mathrm{a} & \text { Ständerstrang a } & p & \text { Polpaarzahl } \\ \mathrm{B} & \text { Induktion } & \text { sat } & \text { sättigungsbedingt } \\ \mathrm{b} & \text { Ständerstrang b } & \text { schr } & \text { Schrägung } \\ \mathrm{Cu} & \text { Kupfer } & \mathrm{sr} & \text { Schrägung } \\ \mathrm{c} & \text { Ständerstrang c } & \mathrm{t} & \text { Tangentialkomponente } \\ \mathrm{Fe} & \text { Eisen } & \mathrm{x} & \text { Richtung } x \text {-Koordinate } \\ \mathrm{i} & \text { induziert } & \mathrm{y} & \text { Richtung } y \text {-Koordinate } \\ \mathrm{j} & \text { Joch } & \mathrm{z} & \text { Richtung } z \text {-Koordinate } \\ \mathrm{s} & \text { Stab } & \mathrm{z} & \text { Zahn } \\ \mathrm{r} & \text { Richtung Radialkoordinate } & \delta & \text { Luftspalt } \\ \mathrm{r} & \text { Ring } & \mu & \text { räumliche Ordnungszahl } \\ \mathrm{h} & \text { Hauptfeldanteil } & \nu & \text { räumliche Ordnungszahl } \\ \sigma & \text { Streufeldanteil } & & \\ \text { mag } & \text { magnetisch } & & \end{array}$




\section{Kurzfassung}

In elektrischen Maschinen werden aufgrund der über dem Umfang periodisch wiederkehrenden Geometrien in der Regel wenige signifikante Einzeltöne erregt. Da derartige Geräuschspektren für den Menschen lästig erscheinen, werden zum Beispiel bei Ventilatoren die Schaufeln ungleichmäßig über dem Umfang angeordnet. Damit wird ein angenehmeres breiteres Geräuschspektrum mit reduzierter Lautstärke der dominierenden Einzeltöne erreicht.

Die vorliegende Arbeit beschäftigt sich damit, das Verfahren einer unregelmäßigen Anordnung der Ventilatorschaufeln auf zum Beispiel die Nutpositionen und -formen von Käfigläufern in Induktionsmaschinen zu übertragen. Hierzu wird zunächst ein Formelwerk zur Berechnung der geräuschanregenden magnetischen Kräfte und Drehmomente aufgebaut, welches insbesondere für unregelmäßig angeordnete oder geformte Nuten gilt.

Durch analytische Überlegungen sowie numerische Optimierungen, welche wiederum auf der analytischen Formulierung der wesentlich zum Geräusch einer Maschine beitragenden Effekte basieren, lassen sich optimierte unregelmäßige Geometrien finden. Anhand beispielhaft ausgewählter optimierter Geometrien werden insbesondere einige Möglichkeiten zur Beeinflussung des Geräuschs von Induktionsmaschinen mit Käfigläufern untersucht. Die Beispiele sind dabei real vermessen beziehungsweise analytisch oder numerisch berechnet. Dabei zeigt die Gegenüberstellung der errechneten Werte mit den Messungen die Eignung der Berechnungsmethoden.

Zusätzlich zur Variation der Nutpositionen und -formen im Läufer wird eine auf den Zahnhöhen des Ständers basierende Methode zur Variation der Luftspaltweite über dem Umfang der Maschine hergeleitet und ebenfalls anhand einiger ausgewählter Beispiele untersucht.

Im Ergebnis zeigt sich, dass mit Hilfe der Variation der Läufernutpositionen und -formen für einige Maschinen ein deutlich angenehmeres Geräusch erzielbar ist. Für die meisten Geometrien ist hingegen kein merklich angenehmeres Geräusch erreichbar. Keine Verbesserung bezüglich des Geräuschs lässt sich durch die Variation der Luftspaltweite beobachten. Diese Variation ist dennoch sinnvoll, um bei Maschinen mit niedriger läuferseitiger Felderregung, also insbesondere bei Synchronreluktanzmaschinen, viele Induktionsoberwellen abzuschwächen.

\section{Schlagworte}

Geräusch, Induktionsmaschine, Luftspaltweite, Modulation, ungleichmäßiger Nutabstand, Nutposition 


\section{Abstract}

Due to periodically repeating geometric elements in electric machines, usually only a few significant single tones will be emitted. As such noise spectra feel uncomfortable for human beings, for example fans are built with uneven blade spacings. This results in a more comfortable wider noise spectra with quieter dominant single tones.

This thesis deals with the idea to transfer the method of uneven blade spacings for example to the slot positions or geometries of cage rotors in induction machines. Therefore, a set of formulas to calculate the noise-exciting magnetic forces and torques of machines with uneven slot spacings and slot geometries will be developed.

By analytic considerations as well as numeric optimizations, which are based on the mathematical description of the main noise-causing effects in electric machines, optimized uneven geometries can be found. Based on selected examples with optimized geometries, several methods to influence the sound of cage induction machines will be investigated. Thereby, the examples will be measured respectively calculated analytically or numerically. The comparison of measurements and calculations shows applicability of the calculation methods.

In addition to the variation of the slot positions and geometries, a method to vary the air gap width by use of different stator tooth heights will be developed. It will also be analyzed with the help of selected examples.

The results show, that for some machines a much more comfortable sound can be achieved by varying the slot positions and geometries. But, for most geometries no appreciable more comfortable sound can be reached. No improvement of the sound can be observed when using the variation of the air gap width. Nevertheless, this method can be used to reduce harmonics of the magnetic flux density of machines with low field excitation of the rotor, that means especially synchronous reluctance machines.

\section{Key words}

Air gap width, induction machine, modulation, noise, slot position, uneven slot pitch 


\section{Einleitung}

Unter anderem aufgrund der Vielzahl elektrischer Maschinen, welche heute in vielen industriellen Arbeitsumgebungen zu finden sind, gehört die von diesen Maschinen emitierte Schallleistung zu den wesentlichen Kriterien bei ihrer Dimensionierung. Hierbei steht nicht nur die reine Schallleistung im Fokus, welche im Übrigen gegenüber anderen Verlustleistungen in elektrischen Maschinen vernachlässigbar ist, sondern auch die Ausprägung des Geräuschspektrums, das bei gleicher Schallleistung einen Einfluss auf die subjektiv von Menschen empfundene Lautheit hat.

Elektrische Maschinen weisen in der Regel Geometrieelemente auf, die sich über dem Umfang periodisch wiederholen. Dies sind bei Induktionsmaschinen zum Beispiel die Ständerund Läufernuten, aber auch die Wicklungszonen. Ausgehend von diesen regelmäßigen Strukturen ergeben sich im Betrieb im Luftspalt magnetische Kraftwellen mit diskreten räumlichen Ordnungszahlen und Frequenzen. Diese diskreten Kraftwellen regen strukturmechanische Schwingungen an und führen somit zu oft nur wenigen signifikanten Einzeltönen im dadurch abgestrahlten Schall.

Ein derartiges schwach besetztes Geräuschspektrum mit wenigen dominanten Einzeltönen ist, wie zum Beispiel in [9] beschrieben, für den Menschen besonders unangenehm. Daher wäre es wünschenswert, das Geräuschspektrum elektrischer Maschinen bei gleichbleibender - oder auch verminderter - Schallleistung aufzuweiten, um einem Rauschen, was für Menschen deutlich angenehmer ist, nahe zu kommen.

Ähnliche Problematiken aufgrund regelmäßiger geometrischer Strukturen sind zum Beispiel von Ventilatoren bekannt. Bei diesen erzeugt jede einzelne Schaufel beim Passieren eines Beobachtungspunkts ein periodisches Geräusch, das als Fourierreihe dargestellt werden kann. Wird dieses Geräusch als unabhängig von den Luftverwirbelungen der anderen Ventilatorschaufeln betrachtet, so entsteht das resultierende Geräusch des Ventilators aus der Überlagerung der Einzelgeräusche. Diese unterscheiden sich bei gleichen Schaufelformen ausschließlich durch ihre Phasenlagen. Die naheliegendste, nämlich gleichmäßige Anordnung der Ventilatorschaufeln führt dabei zu einer Ausprägung diskreter Einzeltöne, deren Frequenzen einem Vielfachen des Produkts aus Drehzahl und Schaufelzahl entsprechen. Das resultierende Geräusch wäre somit aufgrund des nur schwach besetzten Geräuschspektrums gemäß Abschnitt 2.2 für das menschliche Empfinden relativ lästig.

Um die Anregung nur weniger Einzeltöne zu vermeiden, werden die Schaufeln von Ventilatoren häufig mit unregelmäßigen Abständen über dem Umfang angeordnet. Zur Wahl der genauen Schaufelpositionen sind in mehreren Veröffentlichungen, wie zum Beispiel $[1,4,6,8]$, einige Beispiele gezeigt. Die angewandten Modulationsverfahren sind hierbei entweder eine sinusförmige Anordnung, welche mit der Theorie der Phasenmodulation näherungsweise berechnet werden kann, oder eine zufällige Anordnung. Im letzteren Fall wird das Geräuschspektrum einiger Schaufelanordnungen berechnet oder experimentell 


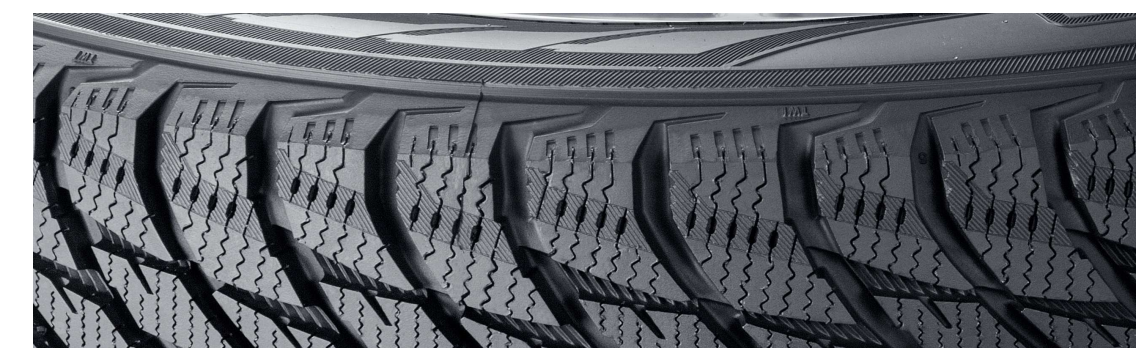

Bild 1.1: Beispiel des Profils eines Winterreifens - Die verschiedenen Pitchlängen lassen sich durch Abzählen der Quernuten innerhalb der Pitches erkennen. (Quelle: Nokian Tyres)

bestimmt, um daraus die Beste auszuwählen. Es handelt sich folglich, zumindest im Fall der Berechnung des Geräuschs, um eine numerische Optimierung.

$\mathrm{Zu}$ beachten ist bei der Anordnung der Ventilatorschaufeln, dass keine Unwuchten entstehen. Diese können zum Beispiel auf einfache Weise dadurch vermieden werden, dass die Schaufeln in mehreren gleichen Gruppen angeordnet werden, welche gleichmäßig über dem Umfang verteilt sind.

Ähnlich wie im Fall der Ventilatoren zeigen Veröffentlichungen wie [16] über die Entwicklung von Fahrzeugreifen, dass auch dort numerische Methoden zur Optimierung der Geometrie Anwendung finden. Wie in Bild 1.1] zu erkennen ist, werden bei Fahrzeugreifen die Pitches, wie die Bereiche zwischen je zwei benachbarten großen Quernuten genannt werden, in verschiedenen, aber in der Regel diskret abgestuften Längen über dem Umfang des Reifens angeordnet.

In dieser Arbeit soll nun das Vorgehen von zum Beispiel Fahrzeugreifen und Ventilatoren auf elektrische Maschinen übertragen und analysiert werden. Es wird untersucht, in wie weit das Geräusch insbesondere von Induktionsmaschinen mit Käfigläufer durch ungleichmäßige Anordnung der Läufer- aber auch Ständernuten derart beeinflusst werden kann, dass sich ein subjektiv angenehmeres Geräusch bei möglichst schwingungsfreiem Drehmoment ergibt.

Hierzu wird zunächst ein Formelwerk zur analytischen Beschreibung der geräuschbildenden Effekte aufgebaut. Damit lassen sich einige Zusammenhänge direkt theoretisch beleuchten. Andere Effekte werden anhand einiger ausgewählter Beispiele in Kapitel 7 untersucht beziehungsweise aufgezeigt.

In der Literatur sind bezüglich der Varation der Maschinengeometrie zur Reduktion der Lautheit und zur Glättung des Drehmoments nur wenige Untersuchungen, wie zum Beispiel die von Morimoto und Morita [17] oder Chitroju [5] zu finden. Diese beziehen sich dabei ausschließlich auf eine Variation der Rotorgeometrie. Beide Arbeiten verfolgen dabei den Ansatz der Bewertung von einfachen manuell vorgegebenen Geometrien. Dies sind in beiden Fällen sogenannte „dual slot Rotoren“, bei denen, wie in Bild 1.2 ersichtlich, zwei diskrete Nutabstände, nämlich die von Rotoren mit zwei verschiedenen Nutzahlen, kombiniert sind. Zudem wird von Chitroju eine sinusförmige Anordnung der Nuten untersucht, wie dies in Bild 5.2 auf Seite 63 zu sehen ist. Dabei richtet er in beiden Fällen den Fokus nicht nur auf das Geräusch, sondern auch auf die Möglichkeit, auf eine Schrägung des Rotors zu verzichten ohne den Anlauf der Maschine zu gefährden. 


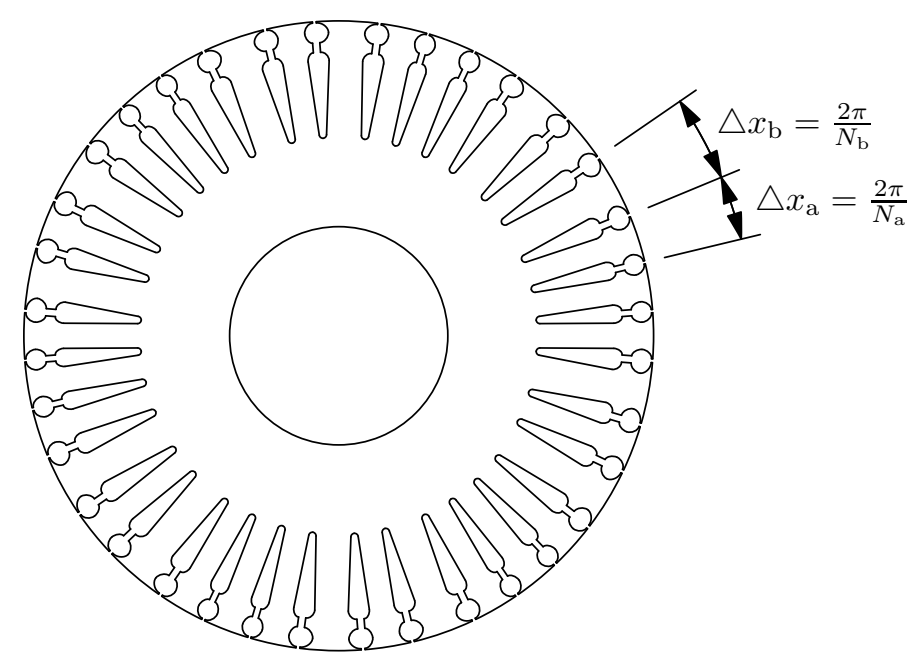

Bild 1.2: Aus den Abständen von $N_{\mathrm{a}}=40$ und $N_{\mathrm{b}}=30$ gleichverteilten Nuten kombinierter „dual slot Rotor" mit $N_{2}=36$ Nuten [17]

Ein weiterer Ansatz, der mit einer unregelmäßigen Nutanordnung im Läufer einhergeht, ist im Patent von Braun und Vollmer [2] dargestellt. Ihr Ansatz zielt bei Verwendung mehrerer elektrisch entkoppelter Läuferwicklungen auf die gezielte Reduktion verschiedener Wicklungsfaktoren eben dieser Wicklungen.

Um das Betriebsverhalten aber insbesondere auch die Schallemission von Induktionsmaschinen zu beeinflussen, ist es grundsätzlich denkbar, jeden Parameter der Nutgeometrie über dem Umfang des Läufers zu variieren. Die nachfolgende Untersuchung beschränkt sich dabei im Läufer auf eine Variation der Nutpositionen und der Nutbreiten. Diese Variationen sind dabei zum Beispiel in Form eines Sinus, Sägezahns oder ähnlichem denkbar. Gleichermaßen bieten sich andere gewissen Regeln folgende Aufbauten, wie die oben genannten „dual slot Rotoren“ an. Um alle diese Möglichkeiten mit einzubeziehen, werden die nachfolgenden Untersuchungen weitgehend allgemein gehalten und beziehen zudem zufällige numerisch zu optimierende Variationen mit ein. 


\section{Grundlagen}

Zum Verständnis der Ausführungen in den nachfolgenden Kapiteln werden an dieser Stelle einige notwendige Grundlagen aufgezeigt.

\subsection{Koordinatensystem}

Das in dieser Arbeit entwickelte Verfahren basiert auf einer Veränderung der Blechschnittgeometrie von Induktionsmaschinen. Um diese zu beschreiben, bedarf es einiger geometrischer Größen und Koordinaten, wovon ein Teil in Bild 2.1 veranschaulicht ist.

Bild 2.1 zeigt die ständerfeste Winkelkoordinate $x_{1}$ und die läuferfeste Winkelkoordinate $x_{2}$. Der Luftspaltdurchmesser $D=2 r$ ergibt sich als Mittelwert aus dem Außendurchmesser $D_{\mathrm{a} 2}=2 r_{\mathrm{a} 2}$ des Läufers und dem Durchmesser $D_{\mathrm{i} 1}=2 r_{\mathrm{i} 1}$ der Ständerbohrung. Die Differenz der beiden Radien entspricht der Luftspaltweite $\delta=r_{\mathrm{i} 1}-r_{\mathrm{a} 2}$.

In der Regel wird ein Zylinderkoordinatensystem mit den Koordinaten $r, x$ und $z$ verwendet, wobei der Ursprung der $z$-Koordinate in der axialen Mitte der Maschine angenommen wird.

Es ist zu beachten, dass positive Induktionen im Luftspalt grundsätzlich in Richtung des Läufers, also entgegen der Radialkoordinate $r$ gerichtet sind.

\subsection{Physiologische Grundlagen des Hörens}

Um beurteilen zu können, wie das akustische Spektrum einer elektrischen Maschine beeinflusst werden sollte, bedarf es eines Grundwissens über die Physiologie des Hörens. Einen

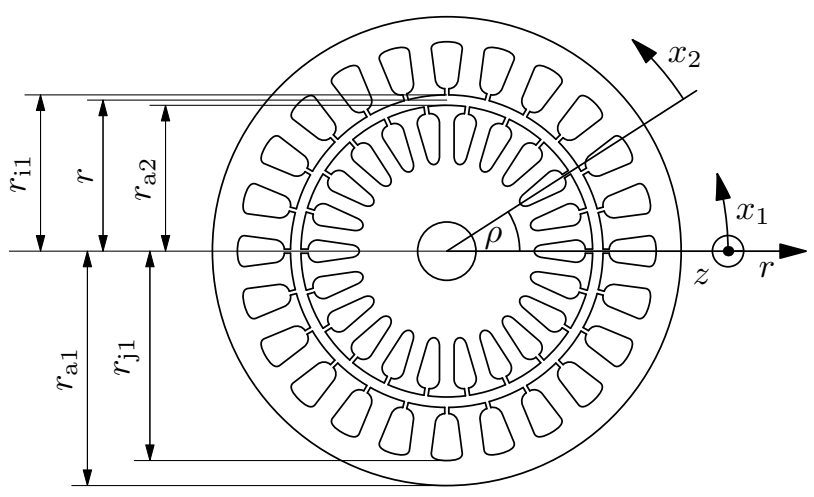

Bild 2.1: Koordinaten 
guten Überblick hierzu liefert [9], auf dessen Inhalt die nachfolgende kurze Zusammenstellung im Wesentlichen basiert.

Menschen können über das Ohr akustische Signale ab einer Frequenz von etwa $16 \mathrm{~Hz}$ wahrnehmen. Die obere Frequenzgrenze des Hörbereichs, welche mit zunehmendem Lebensalter abnimmt, wird in der Literatur mit Werten von $16 \mathrm{kHz}$ bis $20 \mathrm{kHz}$ angegeben.

Innerhalb dieses Bereichs hängt die Wahrnehmung der Lautstärke eines Einzeltons neben dem Schalldruckpegel maßgeblich von der Frequenz des Tons ab. Die maximale Empfindlichkeit weist das Gehör dabei im Bereich von etwa $4 \mathrm{kHz}$ auf. Um diese Frequenzabhängigkeit bei der Angabe eines Schalldruckpegels oder einer Schallleistung zu berücksichtigen, werden häufig bewertete Größen angegeben. Die bekannteste und auch bei den Berechnungen in Kapitel 7 angewandte ist dabei die A-Bewertung nach DIN EN 61672-1.

Neben der Lautstärke der Einzeltöne ist die Wirkung ihrer Kombination von maßgeblicher Bedeutung. Zwei Töne mit dicht nebeneinanderliegenden Frequenzen erscheinen zum Beispiel als Schwankung der Lautstärke. Dieser Effekt vermittelt bei elektrischen Maschinen leicht den Eindruck einer Drehmomentpendelung. Bei einem Referenzton mit $f_{\mathrm{a}}=1000 \mathrm{~Hz}$ tritt er zum Beispiel bei einem zweiten Ton mit der Frequenz $f_{\mathrm{b}}=1000 \mathrm{~Hz} \pm 4 \mathrm{~Hz}$ besonders stark in Erscheinung. Gehen die Frequenzen weiter auseinander, so wird aus der Empfindung einer Schwankung ab etwa $f_{\mathrm{b}}=1000 \mathrm{~Hz} \pm 15 \mathrm{~Hz}$ die Empfindung einer Rauhigkeit. Ab etwa $f_{\mathrm{b}}=1000 \mathrm{~Hz} \pm 75 \mathrm{~Hz}$ sind die Einzeltöne dann direkt als solche wahrnehmbar.

Da das Geräuschspektrum von elektrischen Maschinen auch bei Beschränkung auf die elektromagnetisch angeregten Frequenzen im Allgemeinen mehrere diskrete Einzeltöne enthält, muss für die Bewertung der Lästigkeit des Geräuschs einer Maschine ein objektives Maß für das subjektive Empfinden des gesamten Spektrums herangezogen werden. Gegenüber der Lautheit hängt die Lästigkeit unter anderem von der persönlichen Einstellung der einzelnen Personen zum jeweiligen Geräusch ab. Aufgrund der damit verbundenen Schwierigkeit, die Lästigkeit objektiv zu bewerten, existiert dazu eine Vielzahl von Verfahren. Generell lässt sich dabei feststellen, dass hohe Töne als lästiger wahrgenommen werden als tiefe Töne mit derselben A-bewerteten Schallleistung. Zudem wird ein Spektrum mit lauten Einzeltönen hoher Frequenz gegenüber einem breiteren Spektrum gleicher Gesamtleistung als deutlich lästiger empfunden.

Aus den hier vorgestellten Zusammenhängen lässt sich erkennen, dass ein Ziel der Optimierung der Schallabstrahlung von elektrischen Maschinen eine Aufweitung des Spektrums bei gleicher A-bewerteter Schallleistung sein kann, wobei das Auftreten weniger diskreter nahe beeinanderliegender Einzeltöne vermieden werden sollte.

\subsection{Schrägung}

Um insbesondere nutungsbedingte Erscheinungen in elektrischen Maschinen abzuschwächen, werden diese häufig geschrägt ausgeführt.

Schrägung bedeutet, dass Ständer und Läufer in axialer Richtung, also in Richtung der $z$-Achse gemäß Bild [2.1] um den in Bild 2.2 dargestellen Winkel $\gamma_{\text {schr }}$ gegeneinander verwunden sind. Der daraus resultierende Effekt auf die elektrischen und magnetischen Vorgänge in der Maschine ist dabei nahezu ausschließlich vom Schrägungswinkel $\gamma_{\text {schr }}$ 


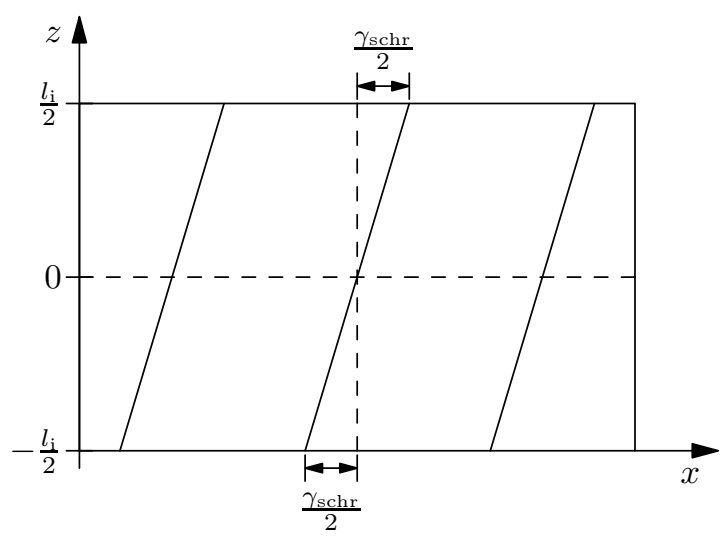

Bild 2.2: Schrägung der Läufernuten bei ungeschrägtem Ständer

abhängig. Dies gilt unabhängig davon, ob nur der Ständer, nur der Läufer oder beide geschrägt ausgeführt werden 1 . Es ist daher ohne wesentliche Beschränkung der Allgemeingültigkeit möglich, grundsätzlich von einem geraden Ständer und einem verwundenen, also geschrägten Läufer auszugehen. Insbesondere bei Käfigläufern wird diese Form aufgrund des geringeren Fertigungsaufwands in der Regel bevorzugt.

Neben der in der Regel erwünschten Auswirkung, nämlich der eingangs erwähnten Abschwächung nutungsbedingter Effekte in elektrischen Maschinen, ergeben sich durch die Schrägung einige parasitäre Erscheinungen. Dies beinhaltet zum Beispiel die in Abschnitt 3.6.1.3 ersichtliche Reduktion der magnetischen Kopplung zwischen Ständer und Läufer, sodass sich bei gleichem Strom, also gleichen Stromwärmeverlusten, ein geringeres Drehmoment ausbildet. Weiterhin entstehen durch die Schrägung, wie in Abschnitt 4.1.1 gezeigt, über der Länge der Maschine phasenverschobene Drehmomente, sodass Torsionsschwingungen angeregt werden. Zudem treten, wie von Jordan und Weis in [13] gezeigt, Eisenquerströme auf, die zusätzliche Verluste zur Folge haben.

\footnotetext{
${ }^{1}$ Die Leiterlänge $\tilde{l}\left(\gamma_{\mathrm{schr}}\right)=\sqrt{l^{2}+\left(r \gamma_{\mathrm{schr}}\right)^{2}}$ und somit auch der Widerstand und die Streuinduktivität steigen mit zunehmender Verwindung an. Dies kann jedoch aufgrund der in der Regel kleinen Werte für $\gamma_{\text {schr }}$ in den meisten Fällen vernachlässigt werden.
} 


\section{Berechnung des Luftspaltfelds}

Methoden zur Berechnung des Luftspaltfelds von Induktionsmaschinen sind vielfach in der Literatur zu finden, zum Beispiel in $[19,28]$. Diese Methoden setzen jedoch in der Regel weitgehende Symmetrie voraus, das heißt gleichverteilte Nuten in Ständer und Läufer.

In diesem Kapitel sollen die Zusammenhänge, die maßgeblich an der Entstehung des Luftspaltfelds beteiligt sind, allgemein, also auch für beliebig angeordnete Nuten beliebiger Breite, auf der Basis von Drehwellen betrachtet werden.

Alle angegebenen Zusammenhänge werden für den idealisierten Fall eines sinusförmigen Ständerstroms einer diskreten Frequenz betrachtet. Das bedeutet, dass zur Berechnung der Maschineneigenschaften für den insbesondere bei Umrichterbetrieb auftretenden Fall eines nicht sinusförmigen Stroms, eine Überlagerung mehrerer sinusförmiger Ströme berechnet werden muss und kann.

Weiterhin sei darauf verwiesen, dass das nachfolgend dargestellte Formelwerk, wie für die Drehwellentheorie üblich, zunächst einen unendlich hohen magnetischen Leitwert des Eisens, also $\mu_{\mathrm{Fe}} \rightarrow \infty$, voraussetzt.

\subsection{Strombelagswellen der Ständerwicklung}

Ein gängiges Verfahren zur Berechnung von elektrischen Maschinen ist die Transformation der in den Ständernuten fließenden Ströme auf einen Strombelag an der Ständerbohrung. Bei Anwendung der Drehfeldtheorie wird dieser Strombelag anschließend mittels einer Fourierreihenentwicklung in eine Summe sinusförmiger Strombelagswellen umgerechnet.

In den Leitern in Nut $(n)$ fließst der zeitabhängige um den Winkel $\varphi_{(n)}$ phasenverschobene Strom

$$
i_{(n)}(t)=\hat{i}_{(n)} \cos \left(\omega_{1} t+\varphi_{(n)}\right),
$$

welcher sich bei gesehnter Ständerwicklung aus einer Überlagerung der Ströme der in der Nut befindlichen Stränge zusammensetzt. Mit der Leiterzahl in der Nut $w_{\mathrm{N}(n)}$ lässt sich dieser Strom in den räumlich über der Nutbreite $b_{\mathrm{N}(n)}$ ausgedehnten Strombelag

$$
a_{(n)}^{*}(t)=\frac{w_{\mathrm{N}(n)} i_{(n)}(t)}{b_{\mathrm{N}(n)}^{*}} \quad \text { im Längenmaß beziehungsweise } \quad a_{(n)}(t)=\frac{w_{\mathrm{N}(n)} i_{(n)}(t)}{b_{\mathrm{N}(n)}}
$$

im Bogenmaßs umrechnen. Dabei gilt

$$
b_{\mathrm{N}(n)}^{*}=r_{\mathrm{i} 1} \cdot b_{\mathrm{N}(n)} .
$$

Eine Fourierreihenentwicklung der räumlichen Ausdehnung dieses Strombelags liefert unter der vereinfachenden Annahme, dass sich die Nut $(n)$ gerade an der Position $x_{1(n)}=0$ 
befindet, die zeitabhängigen Fourierkoeffizienten

$$
\begin{aligned}
2 \cdot \hat{A}_{\mu(n)}(t) & =\frac{1}{\pi} \int_{-\frac{b_{\mathrm{N}(n)}}{2}}^{\frac{b_{\mathrm{N}(n)}}{2}} \frac{w_{\mathrm{N}(n)}}{b_{\mathrm{N}(n)}} \cdot \hat{i}_{(n)} \cos \left(\omega_{1} t+\varphi_{(n)}\right) \cdot \cos \left(\mu x_{1}\right) \mathrm{d} x_{1} \\
& =\frac{w_{\mathrm{N}(n)}}{\pi} \cdot \hat{i}_{(n)} \cos \left(\omega_{1} t+\varphi_{(n)}\right) \cdot \underbrace{\frac{\sin \left(\frac{\left.\mu b_{\mathrm{N}(n)}\right)}{2}\right)}{\frac{\mu b_{\mathrm{N}(n)}}{2}}}_{=\xi_{\mathrm{N} \mu(n)}} \text { mit } \mu \in \mathbb{N} .
\end{aligned}
$$

Dabei ist $\xi_{\mathrm{N} \mu}$ der von Jordan und Lax in [12] eingeführte Nutschlitz- beziehungsweise Breitenfaktor.

Der Strombelag der einzelnen Nut $(n)$ wird somit durch die Fourierreihe

$$
A_{1(n)}\left(x_{1}, t\right)=\sum_{\mu} 2 \cdot \hat{A}_{\mu(n)}(t) \cdot \cos \left(\mu x_{1}\right)
$$

mit zeitabhängigen Fourierkoeffizienten dargestellt. Da die Koeffizienten $\hat{A}_{\mu(n)}(t)$ harmonische Größen sind, lässt sich der Strombelag in zwei gegenläufige Strombelagswellen je räumlicher Ordnungszahl $|\mu|$ überführen. Diese ergeben sich bei nun beliebiger Winkelposition $x_{1(n)}$ der Nut zu

$$
\begin{array}{r}
A_{1 \mu(n)}\left(x_{1}, t\right)=\frac{w_{\mathrm{N}(n)}}{2 \pi} \cdot \xi_{\mathrm{N} \mu(n)} \cdot \hat{i}_{(n)} \cdot \cos \left(\mu x_{1}-\omega_{1} t-\varphi_{(n)}-\mu x_{1(n)}\right) \\
\text { für } \mu=\{ \pm 1, \pm 2, \pm 3, \ldots\}
\end{array}
$$

sowie

$$
A_{1 \mu(n)}\left(x_{1}, t\right)=\frac{w_{\mathrm{N}(n)}}{\pi} \cdot \hat{i}_{(n)} \cdot \cos \left(\omega_{1} t+\varphi_{(n)}\right) \quad \text { für } \quad \mu=0 .
$$

Zur Weiterrechnung bietet die Darstellung der Strombelagswellen durch komplexe Raumzeiger eine elegante Möglichkeit. Hiermit wird die Überlagerung sämtlicher Strombelagswellen aller Nuten zu

$$
\begin{aligned}
& \underline{A}_{1}\left(x_{1}, t\right)=\sum_{\mu \in \mathbb{Z}^{*}} \underbrace{\left[\sum_{n=1}^{N_{1}} \frac{w_{\mathrm{N}(n)}}{2 \pi} \cdot \xi_{\mathrm{N} \mu(n)} \hat{i}_{(n)} e^{-\mathrm{j}\left(-\varphi_{(n)}-\mu x_{1(n)}\right)}\right]}_{=: \hat{A}_{1 \mu} \cdot e^{\mathrm{j} \varphi \mu}} \cdot e^{-\mathrm{j}\left(\mu x_{1}-\omega_{1} t\right)} \\
& +\underbrace{\left[\sum_{n=1}^{N_{1}} \frac{w_{\mathrm{N}(n)}}{\pi} \cdot \hat{i}_{(n)} e^{\mathrm{j} \varphi_{(n)}}\right]}_{=: \hat{A}_{10} \cdot e^{\mathrm{j} \varphi_{0}}} \cdot e^{\mathrm{j} \omega_{1} t}
\end{aligned}
$$

Die Information über die Ausrichtung der Leiter in den Nuten, also ob ein positiver Strom in oder entgegen der Richtung der $z$-Achse fließt, ist dabei in der Phasenverschiebung $\varphi_{(n)}$ der Nutströme enthalten. Der zweite in (3.8) dargestellte Summand, also der Gleichanteil 
des Strombelags, ist der Vollständigkeit halber mit angegeben. Da die Wicklungen elektrischer Maschinen in der Regel derart aufgebaut sind, dass die richtungsbehaftete Summe der Ströme in allen Nuten zu jedem Zeitpunkt Null ist, gilt nahezu immer $\hat{A}_{10}=0$, sodass sich (3.8) entsprechend vereinfacht.

Für den Spezialfall eines symmetrischen Stromsystems, welches sich für $m_{1}=3$ Stränge aus

und

$$
\begin{aligned}
& i_{\mathrm{a}}(t)=\hat{i} \cdot \cos \left(\omega_{1} t+\varphi\right) \\
& i_{\mathrm{b}}(t)=\hat{i} \cdot \cos \left(\omega_{1} t+\varphi-\frac{2 \pi}{3}\right) \\
& i_{\mathrm{c}}(t)=\hat{i} \cdot \cos \left(\omega_{1} t+\varphi-\frac{4 \pi}{3}\right)
\end{aligned}
$$

zusammensetzt, ist es naheliegend, die Spulen der einzelnen Stränge ebenfalls symmetrisch, also beim Beispiel mit $m_{1}=3$ um elektrisch $2 \pi / 3$ verschoben, anzuordnen. Dabei gilt der Zusammenhang zwischen dem mechanischen und dem elektrischen Koordinatensystem $\varphi_{\mathrm{el}}=p \cdot \varphi_{\text {mech }}$. Es entstehen damit Symmetrien, die sich zur Berechnung der Strombelagsamplituden nutzen lassen.

Mit der allgemeinen Annahme einer symmetrischen Bruchlochwicklung entstehen bei $q_{1}=$ $z_{\mathrm{q}} / n_{\mathrm{q}}$ Nuten je Pol und Strang mit

$$
z_{\mathrm{q}}=\frac{N_{1}}{\operatorname{ggT}\left(N_{1}, 2 p m_{1}\right)} \quad \text { und } \quad n_{\mathrm{q}}=\frac{2 p m_{1}}{\operatorname{ggT}\left(N_{1}, 2 p m_{1}\right)}
$$

genau

$$
t=\frac{p}{n_{\mathrm{q}}}
$$

Urwicklungen. Das heißt, dass sich ein- und derselbe Wicklungsaufbau $t$-mal am Umfang wiederholt. Jede dieser $t$ Urwicklungen besteht wiederum aus $m_{1}$ gleich aufgebauten Strängen. Mit diesen Zusatzinformationen ergibt sich für die Strombelagswellen gemäß (3.8) unter der Verwendung des Parameters $\zeta=1$

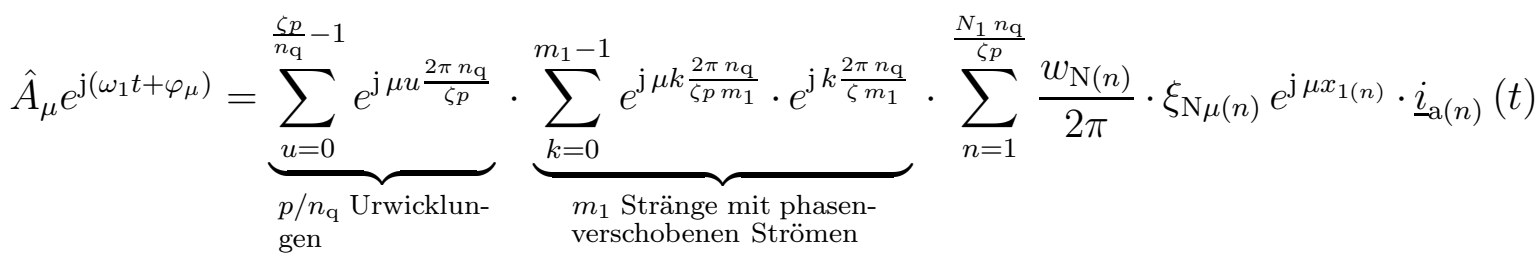

$$
\begin{aligned}
& =\frac{1}{2 \pi} \cdot \underbrace{\sum_{k=0}^{\frac{\zeta p m_{1}}{n_{\mathrm{q}}}-1} e^{\mathrm{j} k \frac{2 \pi n_{\mathrm{q}}}{\zeta m_{1}}\left(\frac{\mu}{p}-1\right)}}_{=: \theta_{\mu}} \cdot \underbrace{\sum_{n=1}^{\frac{N_{1} n_{\mathrm{q}}}{\zeta p}} w_{\mathrm{aN}(n)} e^{\mathrm{j} \mu x_{1(n)}} e^{\mathrm{j} \varphi_{(n)}}}_{=\underline{\xi}_{\mu} \cdot \frac{N_{1} n_{\mathrm{q}}}{\sum_{n=1}^{\zeta p}} w_{\mathrm{aN}(n)}} \cdot \xi_{\mathrm{N} \mu(n)} \hat{i}_{\mathrm{a}(n)} e^{\mathrm{j} \omega_{1} t} .
\end{aligned}
$$

Dabei müssen aufgrund der Ausnutzung der Symmetrie nur der Strom eines Strangs, hier $i_{\mathrm{a}}$ im Strang ,a“, sowie die Leiter desselben Stranges, also die Leiterzahl $w_{\mathrm{aN}}$, betrachtet werden. 


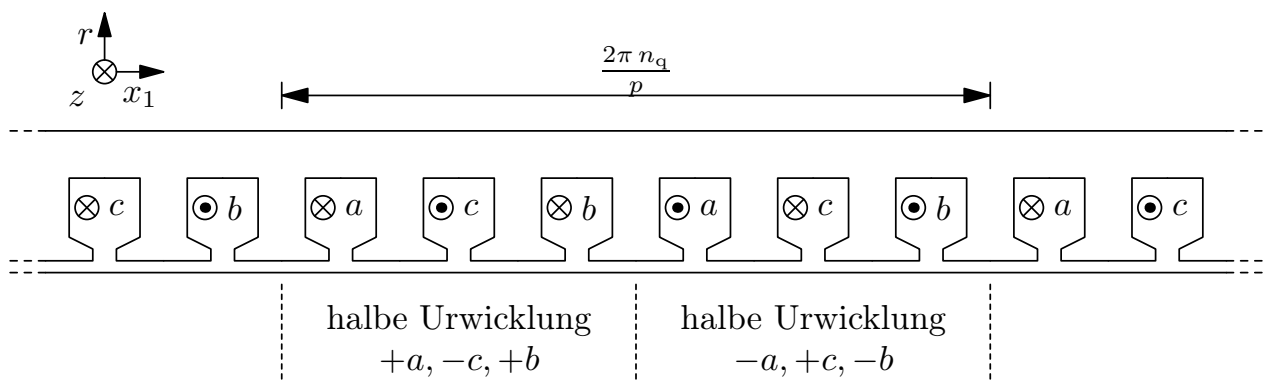

Bild 3.1: Mögliche Antiperiodizität innerhalb einer Urwicklung am Beispiel von $q=1$

Die zu $\theta_{\mu}$ führende Summe in (3.12) ist für $\zeta=1$ nur dann ungleich Null, nämlich $p m_{1} / n_{\mathrm{q}}$, wenn

$$
\frac{n_{\mathrm{q}}}{m_{1}}\left(\frac{\mu}{p}-1\right)=g \quad \Leftrightarrow \quad \mu=p\left(1+\frac{g m_{1}}{n_{\mathrm{q}}}\right) \text { mit } g=\{0, \pm 1, \pm 2, \pm 3, \ldots\}
$$

gilt. Damit ist bekannt, welche räumlichen Ordnungszahlen in der Fourierreihe des Strombelags einer elektrischen Maschine mit entsprechenden Symmetrien auftreten können.

Eine Einschränkung der räumlichen Ordnungszahlen gemäß (3.13) ergibt sich bei einer Antiperiodizität innerhalb einer Urwicklung, wie sie zum Beispiel bei Ganzlochwicklungen auftritt und in Bild 3.1 dargestellt ist. Sie lässt sich in (3.12) durch Wahl des Parameters $\zeta=2$ berücksichtigen. Die räumlichen Ordnungszahlen der möglichen Strombelagswellen werden für diesen Fall auf

$$
\mu=p\left(1+\frac{2 g m_{1}}{n_{\mathrm{q}}}\right) \text { mit } g=\{0, \pm 1, \pm 2, \pm 3, \ldots\}
$$

beschränkt.

Unter der Einschränkung, dass die Zahl der Leiter je Nut $w_{\mathrm{N}(n)}$ für alle $n$ gleich ist, vereinfacht sich (3.12) $\mathrm{zu}$

$$
\hat{A}_{\mu} e^{\mathrm{j} \varphi_{\mu}}(t)= \begin{cases}\frac{m_{1}}{\pi} \cdot \underline{\xi}_{\mu} \bar{\xi}_{\mathrm{N} \mu(n)} w_{1} \sqrt{2} I_{1} \cdot e^{\mathrm{j} \omega_{1} t} & \text { für } \mu \text { gemäß (3.13) bzw. (3.14) } \\ 0 & \text { sonst }\end{cases}
$$

mit

$$
\varphi_{\mu}=\arg \left(\underline{\xi}_{\mu}\right)
$$

Mit den Herleitungen aus diesem Abschnitt lassen sich die Ströme in den Nuten einer elektrischen Maschine letztendlich in Form von Strombelagswellen im Luftspalt als

$$
A_{1}\left(x_{1}, t\right)=-\sum_{\mu} \hat{A}_{\mu} \sin \left(\mu x_{1}-\omega_{1} t+\varphi_{\mu}\right)
$$

darstellen. Dabei ist die Kreisfrequenz aller Strombelagswellen gerade gleich der Kreisfrequenz des Ständerstroms, nämlich

$$
\omega_{\mathrm{A} 1}=\omega_{1} .
$$




\subsection{Wicklungsfaktor der Ständerwicklung}

In (3.12) ist der Wicklungsfaktor der Ständerwicklung

$$
\underline{\xi}_{\mu}=\frac{\sum_{n=1}^{\frac{N_{1} n_{\mathrm{q}}}{p}} w_{\mathrm{aN}(n)} e^{\mathrm{j} \mu x_{1(n)} e^{\mathrm{j} \varphi(n)}}}{\frac{N_{1} n_{\mathrm{q}}}{p} w_{n=1}^{p} w_{\mathrm{aN}(n)}} \quad \text { bzW. } \quad \xi_{\mu}=\frac{\sum_{n=1}^{\frac{N_{1} n_{\mathrm{q}}}{p}} w_{\mathrm{aN}(n)} \cos \left(\mu x_{1(n)}+\varphi_{(n)}-\bar{x}_{1 \mu(n)}\right)}{\frac{\sum_{n=1}^{p}}{w_{\mathrm{aN}(n)}}}
$$

mit $\quad \bar{x}_{1 \mu(n)}=\arg \left(\sum_{n=1}^{\frac{N_{1} n_{\mathrm{q}}}{p}} w_{\mathrm{aN}(n)} e^{\mathrm{j}\left(\mu x_{1(n)}+\varphi_{(n)}\right)}\right)$

bereits gekennzeichnet. Sein Betrag stellt für eine bestimmte räumliche Ordnungszahl $\mu$ das Verhältnis zwischen den Strombelagsamplituden der vorliegenden Wicklung zu den einer den ganzen Pol umfassenden, infinitesimal schmalen konzentrierten Wicklung bei gleicher Windungszahl und gleichem Strom dar. Das Argument des komplexen Wicklungsfaktors spiegelt die räumliche Lage der jeweiligen erregten Strombelagswelle wider.

Aufgrund der Einschränkung von (3.12) auf symmetrische Wicklungen gilt auch (3.19) nur für symmetrische Wicklungen mit beliebiger Nutanordnung und Leiterzahl je Nut.

Bei gleichmäßig angeordneten Ständernuten, deren Winkelpositionen gerade

$$
x_{1(n)}=n \cdot \frac{2 \pi}{N_{1}}+x_{1(0)}
$$

sind, entstehen sogenannte nutharmonische Strombelagswellen.

Aufgrund der $2 \pi$-Periodizität der Kosinusfunktion, beziehungsweise der komplexen Zeigerdarstellung, ergeben sich in (3.19) für alle

$$
\mu=p+g N_{1} \text { mit } g \in \mathbb{Z}
$$

die gleichen Summanden und somit auch die gleichen Wicklungsfaktoren $\xi_{\mu}$.

Die räumliche Ordnungszahl der Hauptwelle, deren Wicklungsfaktor $\xi_{p}$ in der Regel möglichst groß sein sollte, ist in (3.22) enthalten. Daraus folgt, dass auch die Wicklungsfaktoren der nutharmonischen Strombelagswellen ihrem Betrage nach relativ groß sind. Es wird sich in Abschnitt 3.5 zeigen, dass die Induktionswellen nicht nur proportional zum Wicklungsfaktor, sondern auch proportional zu $1 / \mu$ sind, sodass die nutharmonischen Induktionswellen durch Wahl einer möglichst großen Ständernutzahl $N_{1}$ in ihrer Amplitude gering gehalten werden können.

\subsection{Felderregerwellen der Ständerwicklung}

Die Felderregerwellen der Ständerwicklung $V_{1 \mu}\left(x_{1}, t\right)$ ergeben sich durch Integration aus dem Strombelag $A_{1}\left(x_{1}, t\right)$ im Bogenmaß, beziehungsweise seiner Fourierreihendarstellung, 
also den Strombelagswellen gemäß (3.17), über dem Umfang $x_{1}$. Es ergibt sich damit

$$
V_{1}\left(x_{1}, t\right)=\int A_{1}\left(x_{1}, t\right) \mathrm{d} x_{1}=\sum_{\mu} \frac{\hat{A}_{\mu}}{\mu} \cos \left(\mu x_{1}-\omega_{1} t+\varphi_{1}\right)+c(t)
$$

wobei sich die Integrationskonstante $c(t)$ aus der Berechnung der Induktionswellen in Abschnitt 3.5 bestimmt. Die Kreisfrequenzen der Felderregerwellen entsprechen folglich denen des Strombelags, sodass

$$
\omega_{\mathrm{V} 1}=\omega_{\mathrm{A} 1}
$$

gilt.

\subsection{Luftspaltleitwertwellen}

Durch die Nutung von Ständer und Läufer ergeben sich in radialer Richtung über den Luftspalt magnetische Leitwerte, welche von der Winkelkoordinate $x_{1}$ und dem Drehwinkel des Läufers abhängig sind. Unter Anwendung der Drehwellentheorie lässt sich diese Leitwertverteilung als Leitwertwellen darstellen.

Bei regelmäßig angeordneten Nuten besitzen die Luftspaltleitwertwellen überwiegend Ordnungszahlen, welche sich als Kombinationen aus den Nutzahlen des Ständers $N_{1}$ und des Läufers $N_{2}$ ergeben. Dabei sind die Frequenzen im Wesentlichen proportional zur Läufernutzahl $N_{2}$ und zur mechanischen Drehzahl $n$ beziehungsweise Winkelgeschwindigkeit $\Omega$ des Läufers. Es ist zu erwarten, dass der Übergang zu einer unregelmäßigen Anordnung der Läufer- oder auch der Ständernuten durch eine Aufweitung des Spektrums der Leitwertwellen einen signifikanten Einfluss auf das Luftspaltfeld hat.

Zur Berechnung dieses Einflusses bedarf es einer Funktion, die die Luftspaltleitwertwellen, also insbesondere die Leitwerteinbrüche im Bereich der Nutschlitze, möglichst gut beschreibt. In der Literatur sind dazu vielfältige Ansätze zu finden, wobei die von Kolbe [14] nach numerischer Feldberechnung empirisch ermittelte Ersatzfunktion in mehreren Arbeiten [23,34] als am genauesten herausgestellt wurde und dem Vergleich mit eigenen Finite-Elemente-Berechnungen standhielt. Daher soll diese Ersatzfunktion im Folgenden verwendet werden.

Die von Kolbe in [14] ermittelte Ersatzfunktion für den Luftspaltleitwert stellt den Zusammenhang einer homogenen Felderregung, wie diese in Bild $4.2 b$ dargestellt ist, zur Radialkomponente der Luftspaltinduktion im Bereich der Nutschlitze eines Hauptelements dar. Vereinfachend wird dabei angenommen, dass nur ein Hauptelement genutet und das andere ideal glatt ist.

Da die Ersatzfunktion zur Beschreibung der Luftspaltleitwertverteilung auf einer homogenen Felderregung basiert, ist diese insbesondere bei Dominanz von Felderregerwellen niedriger räumlicher Ordnungszahlen mit Periodenweiten deutlich oberhalb der Nutschlitzbreite gültig. Bei diesen ist die Felderregung im Bereich eines Nutschlitzes annähernd homogen. Mit zunehmender Inhomogenität der Felderregung im Bereich der Nuten ist hingegen eine abnehmende Genauigkeit zu erwarten.

Kolbe gibt als Ersatzfunktion der Leitwertverteilung einer einseitig genuteten Maschine 


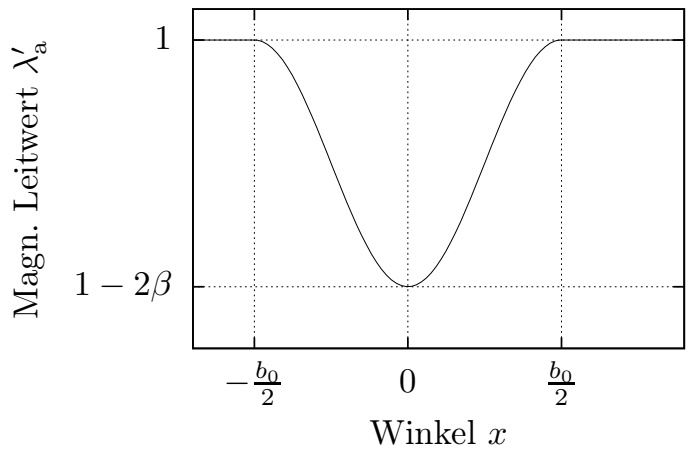

(a) Halbgeschlossene Nuten

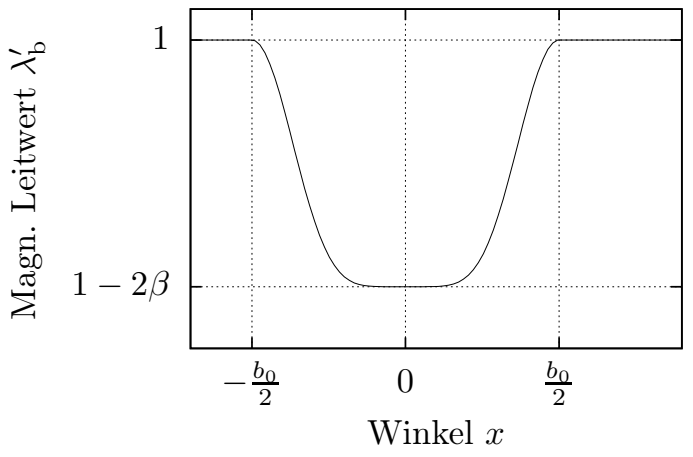

(b) Offene Nuten

Bild 3.2: Verlauf des bezogenen Luftspaltleitwerts im Bereich der Nuten mit $\lambda^{\prime}=\lambda \frac{\delta}{\mu_{0}}$

für halbgeschlossene Nuten

$$
\lambda_{\mathrm{a}}(\alpha)=\frac{\mu_{0}}{\delta} \cdot \begin{cases}1-\beta-\beta \cos \left(\frac{2 \pi}{b_{0}} \alpha\right) & \text { für }-\frac{b_{0}}{2} \leq \alpha \leq \frac{b_{0}}{2} \\ 1 & \text { sonst }\end{cases}
$$

und für offene Nuten

$$
\lambda_{\mathrm{b}}(\alpha)=\frac{\mu_{0}}{\delta} \cdot \begin{cases}1-2 \beta-2 \beta \sin ^{6}\left(\frac{\pi}{b_{0}} \alpha\right) & \text { für }-\frac{b_{0}}{2} \leq \alpha \leq \frac{b_{0}}{2} \\ 1 & \text { sonst }\end{cases}
$$

an. Dabei ist

$$
b_{0}=b_{\mathrm{N}}\left[1+\left(0,8+10^{-4}\left(\frac{b_{\mathrm{N}}^{*}}{\delta}-6\right)^{4}\right) \cdot \exp \left(-\frac{1}{8,5}\left(\frac{b_{\mathrm{N}}^{*}}{\delta}-0,9\right)\right)\right]
$$

die Breite, über der sich eine Leitwertabsenkung ergibt, und

$$
\beta=\frac{1}{2}-\frac{1}{\sqrt{4+\left(\frac{b_{\mathrm{N}}^{*}}{\delta}\right)^{2}}}
$$

die bereits im Jahr 1900 von Carter [3] verwendete Amplitude der Leitwertvariation.

Sowohl die in Bild 3.2a dargestellte Verteilung $\lambda_{\mathrm{a}}(\alpha)$ als auch die in Bild 3.2b dargestellte Verteilung $\lambda_{\mathrm{b}}(\alpha)$ gelten nur für einige Einschränkungen der Geometrie der Nut. Eine allgemeingültige Darstellung erlaubt die Überlagerung zu

$$
\lambda(\alpha)=a \lambda_{\mathrm{a}}(\alpha)+b \lambda_{\mathrm{b}}(\alpha),
$$

wobei die Gewichtsfunktion $a\left(b_{\mathrm{N}}^{*}, \delta\right)$ empirisch zu

$$
a\left(b_{\mathrm{N}}^{*}, \delta\right)= \begin{cases}\exp \left(-\frac{1}{6}\left(\frac{b_{\mathrm{N}}^{*}}{\delta}-1\right)\right) & \text { für } \frac{b_{\mathrm{N}}^{*}}{\delta} \geq 10,6 \\ \sin ^{4}\left(\frac{\pi}{2} \frac{\left.19-\frac{b_{\mathrm{N}}^{*}}{\delta}\right)}{18}\right) & \text { für } \frac{b_{\mathrm{N}}^{*}}{\delta}<10,6\end{cases}
$$


bestimmt wurde. Außerdem gilt

$$
b\left(b_{\mathrm{N}}^{*}, \delta\right)=1-a\left(b_{\mathrm{N}}^{*}, \delta\right) .
$$

Die Leitwertverteilung einer einseitig genuteten Maschine mit beliebig vielen Nuten lässt sich aus (3.25) und (3.26) abschnittweise zusammensetzen und anschließend als Fourierreihe entwickeln.

Alternativ bietet sich die Darstellung des Leitwerteinbruchs jeder einzelnen der $N$ Nuten am Umfang der Maschine durch die für $\nu \in \mathbb{N}^{*}$ gültigen Fourierkoeffizienten

$$
\hat{\lambda}_{\nu(n)}=\frac{-\mu_{0}}{\delta} \frac{\beta}{\pi} \sin \left(\frac{b_{0(n)} \nu}{2}\right) \cdot\left[\begin{array}{l}
a \cdot \frac{8 \pi^{2}}{4 \pi^{2} \nu-b_{0(n)}^{2} \nu^{3}}+\ldots \\
\ldots+b \cdot \frac{8 b_{0(n)}^{6} \nu^{6}-424 \pi^{2} b_{0(n)}^{4} \nu^{4}+5312 \pi^{4} b_{0(n)}^{2} \nu^{2}-12096 \pi^{6}}{b_{0(n)}^{6} \nu^{7}-56 \pi^{2} b_{0(n)}^{4} \nu^{5}+784 \pi^{4} b_{0(n)}^{2} \nu^{3}-2304 \pi^{6} \nu}
\end{array}\right]
$$

sowie die Verminderung des Gleichanteils

$$
\Delta \lambda_{0(n)}=\frac{-\mu_{0}}{\delta} \frac{\beta}{\pi} \cdot \frac{b_{0(n)}}{2} \cdot\left[a+\frac{21 b}{8}\right]
$$

an. Aus dieser Darstellung lassen sich die Fourierkoeffizienten der resultierenden Leitwertverteilung

$$
\hat{\lambda}_{\nu} \cdot e^{-\mathrm{j} \varphi_{\lambda \nu}}=\sum_{n=1}^{N} \hat{\lambda}_{\nu(n)} \cdot e^{\mathrm{j}\left(\nu x_{(n)}\right)}
$$

sowie der resultierende Gleichanteil

$$
\lambda_{0}=\frac{\mu_{0}}{\delta}+\sum_{n=1}^{N} \Delta \lambda_{0(n)}
$$

bestimmen.

Mit der bisherigen Beschränkung auf einseitig genutete Maschinen ist es möglich, zunächst die Luftspaltleitwertwellen $\lambda_{1}\left(x_{1}\right)$ bei ausschließlicher Ständer- und anschließend $\lambda_{2}\left(x_{2}\right)$ bei ausschließlicher Läufernutung zu berechnen. Die resultierende Leitwertverteilung $\lambda\left(x_{1}, \rho\right)$ einer beidseitig genuteten Maschine ergibt sich nach Reihenschaltung von

$$
\lambda_{1}\left(x_{1}\right) \quad \text { und } \quad \lambda_{2}\left(x_{1}, \rho\right)=\lambda_{2}\left(x_{2}\right) \text { mit } x_{2}=x_{1}-\rho,
$$

wobei $\rho$ der in Bild 2.1 eingezeichnete Drehwinkel des Läufers ist.

Da die Leitwerte nach Kolbe auf die volle Luftspaltweite bezogen sind, wäre eine direkte Reihenschaltung fehlerhaft. Um dies zu umgehen, sind die Leitwertverteilungen zunächst durch Reihenschaltung mit dem negativen Leitwert der halben Luftspaltweite

$$
-\lambda_{\delta 2}=-\frac{2 \mu_{0}}{\delta}
$$

auf

$$
\tilde{\lambda}_{1}\left(x_{1}\right)=\frac{\lambda_{1}\left(x_{1}\right) \cdot \frac{-2 \mu_{0}}{\delta}}{\lambda_{1}\left(x_{1}\right)-\frac{2 \mu_{0}}{\delta}} \quad \text { und } \quad \tilde{\lambda}_{2}\left(x_{1}, \rho\right)=\frac{\lambda_{2}\left(x_{1}, \rho\right) \cdot \frac{-2 \mu_{0}}{\delta}}{\lambda_{2}\left(x_{1}, \rho\right)-\frac{2 \mu_{0}}{\delta}}
$$


zu vergrößern.

Die Reihenschaltung der Leitwertverteilungen ergibt sich damit zu

$$
\lambda\left(x_{1}, \rho\right)=\frac{\tilde{\lambda}_{1}\left(x_{1}\right) \cdot \tilde{\lambda}_{2}\left(x_{1}, \rho\right)}{\tilde{\lambda}_{1}\left(x_{1}\right)+\tilde{\lambda}_{2}\left(x_{1}, \rho\right)} .
$$

Bei Fourier-Reihendarstellung der Leitwertverteilungen enthält (3.39) trigonometrische Reihen im Zähler und im Nenner, womit sich gemäß Edrei und Szegö [7] auf einfache Weise kein wiederum als trigonometrische Reihe dargestelltes Ergebnis bilden lässt.

Um dies zu umgehen, bietet sich bei numerischen Untersuchungen, wie auch von Ponick [23] durchgeführt, alternativ eine Reihenschaltung der Luftspaltleitwerte an diskreten Stützpunkten über $x_{1}$ mit ebenfalls diskreten Läuferwinkeln $\rho$ und anschließender zweidimensionaler schneller Fouriertransformation an, um eine orts- und zeitabhängige Fourierreihendarstellung der Luftspaltleitwertverteilung zu erhalten.

Es ergibt sich damit eine Leitwertverteilung der Form

$$
\lambda\left(x_{1}, \rho\right)=\sum_{\nu=-\infty}^{\infty} \sum_{\mu=0}^{\infty} \underbrace{\hat{\lambda}_{\nu \mu} \cos \left(\nu x_{1}-\mu \rho+\varphi_{\lambda \nu \mu}\right)}_{=: \lambda_{\nu \mu}\left(x_{1}, \rho\right)},
$$

welche sich mit den Beziehungen $\rho=\Omega t+\rho_{0}$ und $\omega_{\mu}=\mu \Omega$ leicht in

$$
\lambda\left(x_{1}, t\right)=\sum_{\nu=-\infty}^{\infty} \sum_{\mu=0}^{\infty} \hat{\lambda}_{\nu \mu} \cos \left(\nu x_{1}-\omega_{\mu} t+\varphi_{\lambda \nu \mu}\right)
$$

überführen lässt.

Näherungsweise lässt sich die Leitwertverteilung jedoch auch direkt aus (3.38) und (3.39) bestimmen, indem die unendlichen Reihen im Nenner nach dem ersten, also dem konstanten Glied $\lambda_{10}$ beziehungsweise $\lambda_{20}$, abgebrochen werden. Es ergibt sich so die Näherung

$$
\begin{gathered}
\lambda\left(x_{1}, \rho\right) \approx \\
\lambda^{\prime \prime}\left(x_{1}, \rho\right)=\underbrace{\frac{\frac{-2 \mu_{0}}{\delta \lambda_{10}-2 \mu_{0}} \cdot \frac{1}{\delta \lambda_{20}-2 \mu_{0}}}{\delta \lambda_{10}}+\frac{\lambda_{20}}{\delta \lambda_{10}-2 \mu_{0}}+\infty}_{=: \frac{\lambda_{0}^{\prime \prime}}{\lambda_{10} \cdot \lambda_{20}}} \sum_{\nu=0}^{\infty} \sum_{\mu=0}^{\infty} \hat{\lambda}_{1 \nu} \hat{\lambda}_{2 \mu} \underbrace{\cos \left(\nu x_{1}+\varphi_{\lambda \nu}\right)}_{\text {Ständer }} \cdot \underbrace{\cos \left(\mu x_{1}-\mu \rho+\varphi_{\lambda \mu}\right)}_{\text {Läufer }},
\end{gathered}
$$

deren Genauigkeit in Abschnitt 5.1 untersucht wird. Dabei wird sich zeigen, dass diese Näherung zur vergleichenden Bewertung verschiedener Spektren je nach Nutgeometrie weitgehend aussagekräftig ist.

Es ergeben sich aus (3.42) resultierend die Luftspaltleitwertwellen

$$
\lambda\left(x_{1}, \rho\right)=\sum_{\kappa=-\infty}^{\infty} \sum_{\mu=0}^{\infty} \hat{\lambda}_{\kappa \mu} \cos \left(\kappa x_{1}-\omega_{\mu} t+\varphi_{\kappa}\right)
$$

mit den räumlichen Ordnungszahlen

$$
\kappa=\mu \pm \nu \text { also } \kappa \in \mathbb{Z}
$$


und bei Rotation des Läufers mit $\rho=\Omega t$ den Kreisfrequenzen

$$
\omega_{\mu}=\mu \Omega
$$

welche gerade denen gemäß (3.41) entsprechen.

Luftspaltleitwertwellen, welche in Richtung der Drehung des Läufers umlaufen, ergeben sich folglich für $\mu>\nu$ und Wellen, welche dem Läuferdrehsinn entgegen laufen, für $\mu<\nu$. Allgemein, also bei beliebiger Anordnung der Ständer- und Läufernuten, kann das Frequenzspektrum des Luftspaltleitwerts dabei sämtliche Vielfache der Winkelgeschwindigkeit des Läufers enthalten.

Eine Einschränkung der räumlichen und zeitlichen Ordnungszahlen ergibt sich für die unmodulierte Anordnung der Ständernuten gemäß (3.21) sowie analog der Läufernuten. Hierfür wird mit (3.34) ersichtlich, dass ausschließlich die räumlichen Ordnungszahlen

$$
\nu=k_{\nu} N_{1} \quad \text { und } \quad \mu=k_{\mu} N_{2} \quad \text { mit } \quad k_{\nu}, k_{\mu} \in \mathbb{N}
$$

und damit

$$
\kappa=k_{\mu} N_{2} \pm k_{\nu} N_{1}
$$

sowie die Kreisfrequenzen

$$
\omega_{\mu}=k_{\mu} N_{2} \cdot \Omega \quad \text { mit } \quad k_{\mu} \in \mathbb{N}
$$

auftreten.

\subsubsection{Schrägung}

Bei Schrägung der Maschine, die hier ausschließlich als läuferseitige Schrägung betrachtet werden soll, liegen die läuferseitigen Einflüsse auf die Leitwertverteilung über der Länge der Maschine tangential verschoben. Damit bekommt die Leitwertverteilung eine Abhängigkeit von der axialen Koordinate $z$.

Es ergibt sich

$$
\begin{aligned}
\lambda\left(x_{1}, \rho, z\right) & =\lambda\left(x_{1}, \rho+\frac{\gamma_{\mathrm{schr}}}{l_{\mathrm{i}}} \cdot z\right) \\
& =\lambda_{0}+\sum_{\nu=1}^{\infty} \sum_{\mu=1}^{\infty} \hat{\lambda}_{\nu \mu} \cos (\nu x_{1}-\mu \rho-\underbrace{\mu \gamma_{\mathrm{schr}}}_{=: \sigma} \frac{z}{l_{\mathrm{i}}}+\varphi_{\lambda \nu \mu})
\end{aligned}
$$

mit der zusätzlich eingeführten Variable $\sigma$, welche proportional zum Schrägungswinkel $\gamma_{\text {schr ist. }}$

Bei Anwendung der Näherung aus (3.42) ergibt sich die Leitwertverteilung damit zu

$$
\lambda\left(x_{1}, \rho, z\right) \approx \lambda^{\prime \prime}\left(x_{1}, \rho, z\right)=\frac{\lambda_{0}^{\prime \prime}}{\lambda_{10} \cdot \lambda_{20}} \cdot \lambda_{1}\left(x_{1}\right) \cdot \lambda_{2}\left(x_{1}, \rho+\frac{\gamma_{\mathrm{schr}}}{l_{\mathrm{i}}} \cdot z\right)
$$

Bei Einbeziehung einer Schrägung des Läufers liegt es nahe, nicht nur die Läufernutpositionen, sondern auch die Schrägung von Nut zu Nut zu variieren. Dieser Ansatz führt zu 


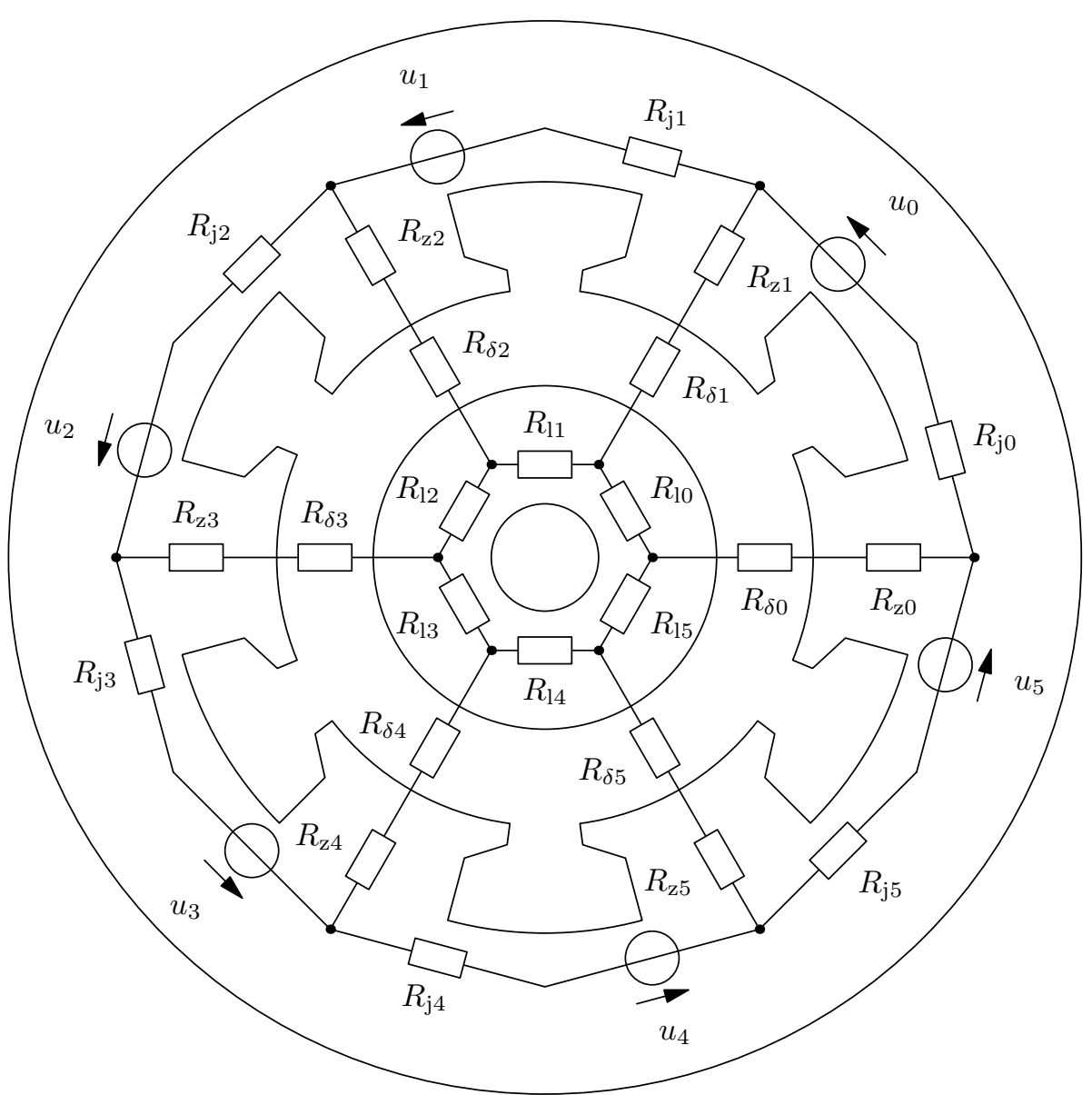

Bild 3.3: Vereinfachtes Ersatzschaltbild des Magnetkreises einer elektrischen Maschine

verschiedenen Modulationen der Nutpositionen in den einzelnen Läuferblechen und somit zu ungleichen Blechgeometrien über der Maschinenlänge. Obwohl dieser Ansatz akademisch sehr interessant ist, soll er aufgrund des erhöhten Fertigungsaufwands nicht weiter betrachtet werden.

\subsubsection{Magnetische Spannung über den Eisenwegen}

Neben dem magnetischen Leitwert, beziehungsweise der magnetischen Spannung über dem Luftspalt, ergibt sich eine magnetische Spannung $V_{\mathrm{Fe}}$ über den Eisenwegen, also, wie in Bild 3.3 vereinfacht dargestellt, in den Ständer- und Läuferzähnen in radialer Richtung sowie im Ständer- und Läuferjoch in Umfangsrichtung.

Ist die magnetische Spannung $V_{\mathrm{Fe}, \mathrm{Zahn}}$ längs der Zähne dominierend in der gesamten magnetischen Spannung im Eisen $V_{\mathrm{Fe} \text {,gesamt }}$, so kann der Einfluss von $V_{\mathrm{Fe}, \text { gesamt }}$ auf das Luftspaltfeld mit guter Näherung, wie üblich, durch einen aufgeweiteten Luftspalt berücksichtigt werden [30]. Eventuelle Sättigungseinflüsse lassen sich dabei vereinfacht, wie von Nürnberg gezeigt [21], durch den Abplattungsfaktor $\alpha$ in den Luftspalt mit einbeziehen.

Um die magnetischen Spannungsabfälle in den Jochen von Ständer- und Läufer korrekt abbilden zu können, reicht diese einfache Aufweitung des Luftspalts hingegen nicht aus. 
Damit unter anderem die verschiedenen räumlichen Ordnungszahlen der Felderreger- und später Induktionswellen mit den unterschiedlich weiten Wegen in den Jochen berücksichtigt werden könnten, müssten ordnungszahlabhängige Jochleitwertwellen eingeführt werden, welche den magnetischen Widerstand über dem Joch darstellen und zum Beispiel für die Hauptwelle zu zusätzlichen Oberwellen führen.

Mit derartigen Jochleitwertwellen könnten insbesondere alle Wellen mit einer räumlichen Ordnungszahl ungleich derer der Hauptwelle, für die die einfache Luftspaltaufweitung auf $\delta^{\prime \prime}$ in der Regel angepasst ist, präziser abgebildet werden. Dennoch soll zur Vereinfachung im Folgenden von dieser Möglichkeit abgesehen werden. Es wird folglich für die Näherung von einer unendlich hohen magnetischen Permeabilität des Eisens $\mu_{\mathrm{Fe}} \rightarrow \infty$ ausgegangen, sodass in der Realität eventuell geringfügige Korrekturen der Ergebnisse notwendig werden.

Besonders deutlich wird der Einfluss der magnetischen Spannungsabfälle in den Eisenwegen bei signifikanten Sättigungseffekten in den Zähnen oder Jochen. Bei Dominanz der Zahnsättigung der Hauptwelle sind dies zum Beispiel zusätzliche Leitwertwellen, welche von einer Leitwertwelle mit der doppelten räumlichen Ordnungszahl der Hauptwelle und der doppelten Netzfrequenz gemäß (6.1) dominiert werden. Derartige Leitwertwellen müssen je nach Dimensionierung der Maschinen berücksichtigt werden. Obwohl durch die Sättigungsleitwertwellen zusätzliche Frequenzen, also auch zusätzliche Einzeltöne auftreten können, sollen diese zur Vereinfachung in der nachfolgenden Argumentation im Rahmen der Entwicklung des Formelwerks zunächst nicht berücksichtigt werden, lassen sich jedoch später leicht ergänzen, sodass sich keine Beschränkung der Allgemeingültigkeit ergibt.

Zusätzliche auf die Eisenwege zurückzuführende Leitwertwellen ergeben sich bei Inhomogenitäten des für den Fluss zur Verfügung stehenden Eisenquerschnitts. Diese entstehen zum Beispiel durch unterschiedlich breite Zähne bei gleich breiten Nuten über dem Umfang der Maschine. Hierdurch entstehen Leitwertwellen, welche entweder ständer- oder läuferfest wirken.

\subsection{Ständerseitig erregte Induktionswellen}

Die ständerseitig hervorgerufenen Induktionswellen ergeben sich aus einer Multiplikation der Felderregerwellen (3.23) mit den Leitwertwellen (3.41) $\mathrm{zu}$

$$
\begin{aligned}
B_{1}\left(x_{1}, t\right) & =V_{1}\left(x_{1}, t\right) \cdot \lambda\left(x_{1}, t\right) \\
& =\left[\sum_{\mu} \frac{\hat{A}_{\mu}}{\mu} \cos \left(\mu x_{1}-\omega_{1} t+\varphi_{1}\right)+c(t)\right] \cdot \sum_{\kappa} \sum_{\iota} \hat{\lambda}_{\kappa \iota} \cdot \cos \left(\kappa x_{1}-\omega_{\iota} t+\varphi_{\lambda \kappa \iota}\right) \\
& =\sum_{\nu} \sum_{\vartheta} \hat{B}_{\nu \vartheta} \cos \left(\nu x_{1}-\omega_{\vartheta} t+\varphi_{\nu \vartheta}\right)
\end{aligned}
$$

und werden, wie in der Drehwellentheorie üblich, im Luftspalt als rein radial angenommen.

Sind die magnetischen Leitwerte über die Stirnflächen der Maschine hinreichend klein, so kann der Fluss über diese Wege vernachlässigt werden. Damit lässt sich die in (3.23) 
eingeführte Integrationskonstante $c(t)$ bestimmen, sodass

$$
\int_{0}^{2 \pi} B\left(x_{1}, t\right) \mathrm{d} x_{1}=0 \quad \text { und damit für alle } \vartheta \quad \hat{B}_{0 \vartheta}=0
$$

gilt. Die auftretenden Kreisfrequenzen der Luftspaltinduktionswellen ergeben sich mit

$$
\omega_{\iota}=\iota \Omega
$$

gemäß (3.45) unter der Bedingung ausschließlich positiver Werte zu

$$
\omega_{\vartheta}=\omega_{1}+\iota \Omega \quad \vee \quad \omega_{\vartheta}=\left\{\begin{array}{ll}
\omega_{1}-\iota \Omega & \text { für } \omega_{1} \geq \iota \Omega \\
\iota \Omega-\omega_{1} & \text { sonst }
\end{array} \quad \text { mit } \quad \iota \in \mathbb{N} .\right.
$$

Somit können alle Kombinationen aus der positiven oder negativen Kreisfrequenz des Ständerstromes $\omega_{1}$ mit allen positiven oder negativen Vielfachen der Winkelgeschwindigkeit des Läufers $\Omega$ auftreten.

Bei Maschinen mit geschrägtem Läufer ergibt sich aufgrund der über der Länge der Maschine nicht konstanten Luftspaltleitwerte eine Abhängigkeit der ständerseitig erregten Induktion von der $z$-Koordinate. Aus (3.51) wird damit

$$
\begin{aligned}
B_{1}\left(x_{1}, t, z\right) & =V_{1}\left(x_{1}, t\right) \cdot \lambda\left(x_{1}, t, z\right) \\
& =V_{1}\left(x_{1}, t\right) \cdot \lambda\left(x_{1}, t+\frac{\gamma_{\mathrm{schr}}}{\Omega \cdot l_{\mathrm{i}}} \cdot z\right) \\
& =\sum_{\nu} \sum_{\vartheta} \hat{B}_{1 \nu \vartheta} \cdot \cos \left(\nu x_{1}-\omega_{\vartheta} t-\sigma \cdot \frac{z}{l_{\mathrm{i}}}+\varphi_{\nu \vartheta}\right) .
\end{aligned}
$$

\subsection{Läuferrückwirkung}

Jede ständerseitig erregte Induktionswelle $B_{1 \nu}\left(x_{1}, t\right)$, die mit einer relativ zum Läufer unterschiedlichen Winkelgeschwindigkeit umläuft, induziert in den Maschen des Läuferkäfigs eine Spannung. Daraus resultieren Ströme und somit läuferseitige Felderregungen. Nach Modulation durch die Luftspaltleitwertwellen ergeben sich hieraus läuferseitig erregte Induktionswellen $B_{2 \mu}\left(x_{1}, t\right)$, welche erneut zu induzierten Spannungen in den Maschen des Läuferkäfigs und somit zu Felderregungen führen.

Zur Berechnung der Läuferrückwirkung lässt sich der Käfig mit Hilfe des in Bild 3.4 dargestellten Ersatzschaltbilds beschreiben. Dabei sind $L_{\mathrm{s} \sigma}$ und $R_{\mathrm{s}}$ beziehungsweise $L_{\mathrm{r} \sigma}$ und $R_{\mathrm{r}}$ die Streuinduktivitäten und die Widerstände der einzelnen Stäbe beziehungsweise Ringsegmente zwischen zwei benachbarten Stäben. $L_{\delta}$ repräsentiert die Anteile der Selbstinduktivitäten der einzelnen Käfigmaschen, welche auf einem Fluss über den Luftspalt basieren, und $u_{\mathrm{M}}$ die über die Gegeninduktivitäten zu den jeweils anderen Maschen induzierten Spannungen. Durch $u_{\mathrm{i} 1}$ werden die von den ständerseitig erregten Feldwellen induzierten Spannungen dargestellt. 


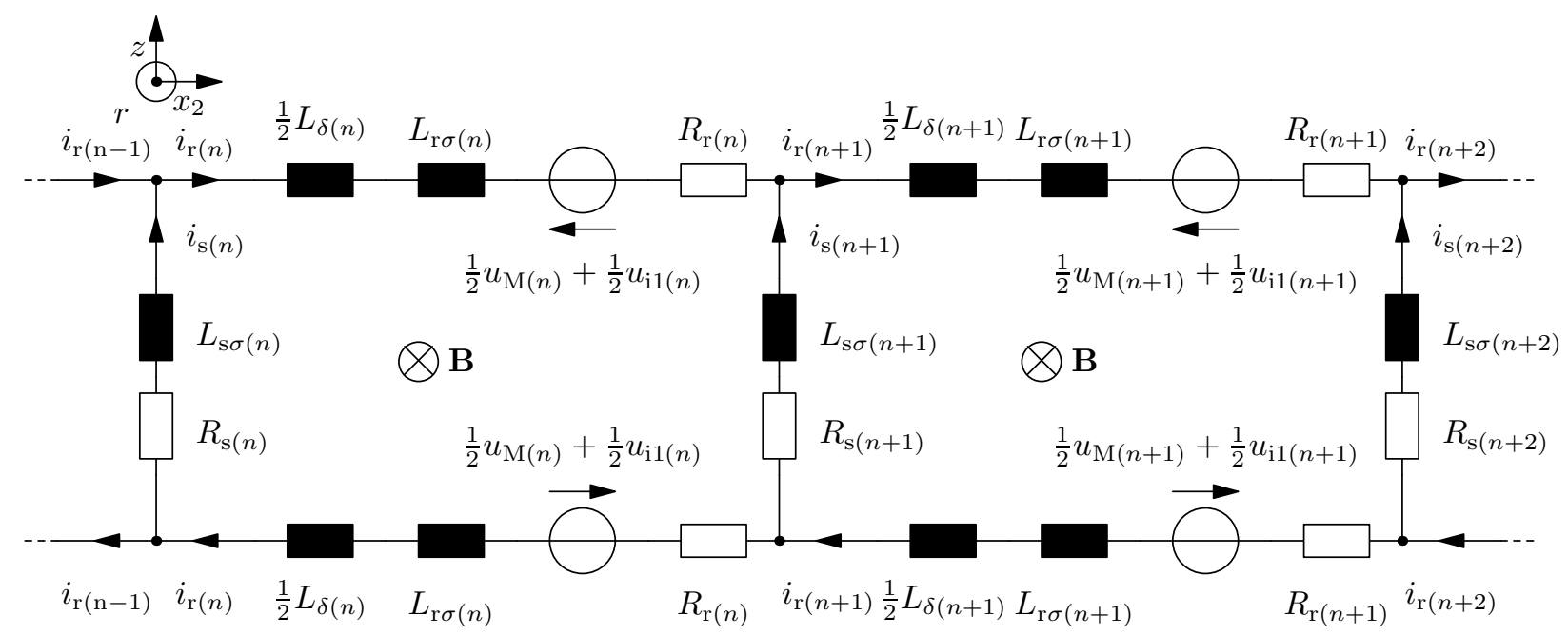

Bild 3.4: Ersatzschaltbild des Käfigs im Läufer

In diesem Abschnitt werden zwei verschiedene Modelle des Läufers aufgestellt. Zum Einen ist dies ein möglichst reales diskretes Modell, in dem jeder einzelner Läuferstab wiederzufinden ist, und zum Anderen ein Modell, in dem der Käfig in ein Kontinuum übergeht. Letzteres soll der Veranschaulichung einzelner Effekte aufgrund zum Beispiel einzelner Feldwellen dienen und ist modellbedingt ungenauer als das diskrete Modell.

\subsubsection{Induzierte Spannung}

Ausgangspunkt der Läuferrückwirkung ist die vom Ständerfeld im Käfig induzierte Spannung $u_{\mathrm{i} 1}$.

Herrührend von der Ständerinduktionswelle

$$
B_{\nu}\left(x_{1}, t\right)=\hat{B}_{\nu} \cos \left(\nu x_{1}-\omega_{1 \mu} t+\varphi_{\nu}\right)=B_{\nu}\left(x_{2}, t\right)=\hat{B}_{\nu} \cos \left(\nu x_{2}-\omega_{2 \nu} t+\varphi_{\nu}\right)
$$

ergibt sich mit der ideellen Länge der Maschine $l_{\mathrm{i}}$ die Spannung

$$
\begin{aligned}
u_{\mathrm{i}(n) \nu}(t) & =-\frac{\mathrm{d} \Phi_{(n)}(t)}{\mathrm{d} t}=-\frac{\mathrm{d}}{\mathrm{d} t} \int_{-\frac{l_{\mathrm{i}}}{2}}^{\frac{l_{\mathrm{i}}}{2}} \int_{x_{2(n)}}^{x_{2(n+1)}} B_{\nu}\left(x_{2}, t\right) r \mathrm{~d} x_{2} \mathrm{~d} z \\
& =\underbrace{\hat{B}_{\nu} r l_{\mathrm{i}} \omega_{2 \nu}}_{=: \hat{u}_{\mathrm{i} \nu}} \underbrace{\frac{2}{\nu} \sin \left(\nu \frac{x_{2(n+1)}-x_{2(n)}}{2}\right)}_{=: \xi_{\mathrm{u}(n) \nu}} \cos \left(\nu x_{2 \mathrm{z}(n)}-\omega_{2 \nu} t+\varphi_{\nu}+\frac{\pi}{2}\right),
\end{aligned}
$$

wobei

$$
x_{2 \mathrm{z}(n)}=\frac{x_{2(n)}+x_{2(n+1)}}{2}
$$

die Zahnposition und

$$
\omega_{2 \nu}=\omega_{1 \mu}-\nu \Omega=\left(1-\frac{(1-s) \nu}{p}\right) \omega_{1 \mu}
$$


die vom Schlupf $s$ abhängige Kreisfrequenz im läuferfesten Koordinatensystem sind.

Es ergibt sich dabei aus (3.59) und den Kreisfrequenzen der Ständerinduktionswellen $\omega_{1 \mu}$ beziehungsweise $\omega_{\vartheta}$ entsprechend (3.54), dass die induzierten Spannungen im Läufer, genau wie auch die Ständerinduktionswellen, Kreisfrequenzen aller Kombinationen aus der positiven oder negativen Kreisfrequenz des Ständerstromes $\omega_{1}$ mit allen positiven oder negativen Vielfachen der Winkelgeschwindigkeit des Läufers $\Omega$ besitzen können.

\subsubsection{Einkopplungsfaktor}

In (3.57) ist der Einkopplungsfaktor $\xi_{\mathrm{u} \nu}$ markiert. Da zur dortigen Berechnung der induzierten Spannung angenommen wurde, dass sämtlicher Fluss von einer bis zur nächsten Nutmitte durch den dazwischenliegenden Zahn tritt, erscheint es legitim, im Folgenden mit der vereinfachten Zahnbreite für infinitesimal schmale Nuten

$$
b_{\mathrm{z}(n)}=x_{2(n+1)}-x_{2(n)}
$$

zu rechnen. Damit wird der Einkopplungsfaktor zu

$$
\xi_{\mathrm{u} \nu}=\frac{2}{\nu} \sin \left(\frac{\nu b_{\mathrm{z}}}{2}\right)=b_{\mathrm{z}} \cdot \frac{\sin \left(\frac{\nu b_{\mathrm{z}}}{2}\right)}{\frac{\nu b_{\mathrm{z}}}{2}}=b_{\mathrm{z}} \cdot \xi_{\mathrm{K} \nu}
$$

beziehungsweise im Fall einer gleichmäßigen Nutverteilung zu

$$
\xi_{\mathrm{u} \nu}=\frac{2}{\nu} \sin \left(\frac{\nu \pi}{N_{2}}\right)=\frac{2 \pi}{N_{2}} \cdot \xi_{\mathrm{K} \nu}
$$

Der Einkopplungsfaktor repräsentiert den Einfluss der Zahnbreiten auf die induzierten Spannungen $u_{\mathrm{i}(n)}$. Er weicht dabei um den Faktor $b_{\mathrm{z}}$ vom Kopplungsfaktor $\xi_{\mathrm{K} \nu}$ ab, welcher üblicherweise in der Literatur Anwendung findet.

Hilfreich sind der Einkopplungs- und der Kopplungsfaktor, um zum Beispiel im weiter unten entwickelten kontinuierlichen Modell des Läufers trotz des Übergangs zu unendlich vielen Läufernuten $N_{2} \rightarrow \infty$ mit infinitesimal kleinen Zahnbreiten $b_{\mathrm{z}} \rightarrow 0$ den Einfluss der realen Zahnbreiten zu berücksichtigen.

\subsubsection{Beeinflussung der induzierten Spannung durch eine Modulation der Läufernutpositionen}

Nachfolgend soll beispielhaft der Einfluss einer sinusförmigen Nutanordnung auf die induzierten Spannungen im Läuferkäfig aufgezeigt werden. Bei dieser Annordnung lässt sich, wie in [8] zu sehen, die aus der Nachrichtentechnik bekannte Theorie der Phasenmodulation anwenden. Ein Beispiel einer sinusförmigen Anordnung ist in Bild 5.2 zu sehen. Es sei angemerkt, dass das nachfolgend gezeigte Vorgehen auch auf andere Größen als die induzierte Spannung übertragen werden kann.

Der Einfluss einer Modulation der Läufernutpositionen auf die induzierte Spannung $u_{\mathrm{i} 1}$ wird im Wesentlichen durch die Zahnbreite $b_{z}$ definiert. Folglich lässt sich dieser durch den Kopplungsfaktor gemäß Abschnitt 3.6.1.1 beschreiben. 
Für das Beispiel einer sinusförmigen Nutanordnung mit $\tilde{b}_{2}$ Perioden am Umfang ergeben sich die Nutpositionen

$$
x_{2(n)}=\frac{2 \pi}{N_{2}} n+\vartheta \sin \left(\tilde{b}_{2} \frac{2 \pi}{N_{2}} n\right) .
$$

Die Abstände je zweier benachbarter Nuten sind folglich

$$
x_{2(n+1)}-x_{2(n)}=b_{\mathrm{z}(n)}=\frac{2 \pi}{N_{2}}+\underbrace{\vartheta}_{=: \Delta \varphi} \underbrace{\sin \left(\tilde{b}_{2} \frac{\pi}{N_{2}}\right)} \cdot \cos \left(\tilde{b}_{2} \frac{\pi}{N_{2}}(2 n+1)\right)
$$

sodass sich der Einkopplungsfaktor mit dem Übergang zur kontinuierlichen Betrachtung $\mathrm{zu}$

$$
\begin{aligned}
\xi_{\mathrm{u} \nu}\left(x_{2}\right) & =\frac{2}{\nu} \sin (\underbrace{\frac{\nu \pi}{N_{2}}+\nu \Delta \varphi \cdot \cos \left(\tilde{b}_{2} x_{2}\right)}_{=\frac{\nu}{2} \cdot b_{\mathrm{z}}\left(x_{2}\right)}) \\
& =\frac{1}{\nu}\left[e^{\mathrm{j}\left(\frac{\nu \pi}{N_{2}}-\frac{\pi}{2}\right)} \cdot e^{\mathrm{j} \nu \Delta \varphi \cdot \cos \left(\tilde{b}_{2} x_{2}\right)}+e^{-\mathrm{j}\left(\frac{\nu \pi}{N_{2}}-\frac{\pi}{2}\right)} \cdot e^{-\mathrm{j} \nu \Delta \varphi \cdot \cos \left(\tilde{b}_{2} x_{2}\right)}\right]
\end{aligned}
$$

ergibt.

Dabei ist $\tilde{b}_{2}$ bei kontinuierlicher Betrachtung gerade gleich der Anzahl $b_{2}$ gleicher Blöcke beziehungsweise Urwicklungen im Läufer. Für den diskret betrachteten Fall muss hingegen berücksichtigt werden, dass zum Beispiel eine sinusförmige Nutanordnung zu unterschiedlich vielen Nuten je Periode führen kann, sodass

$$
b_{2}=\operatorname{ggT}\left(\tilde{b}_{2}, N_{2}\right)
$$

gilt.

Ähnlich dem Vorgehen in [8] kann (3.65) zum Beispiel mit der in [27] gezeigten Beziehung

$$
e^{\mathrm{j} \beta \sin (x)}=\sum_{n=-\infty}^{\infty} J_{n}(\beta) \cdot e^{\mathrm{j} n x}
$$

und der in Bild 3.5 dargestellten Bessel-Funktion

$$
J_{n}(x)=\sum_{k=0}^{\infty} \frac{(-1)^{k}}{k ! \cdot \Gamma(n+r+1)}\left(\frac{x}{2}\right)^{2 k+n} \quad \text { mit } \quad \Gamma(x)=\int_{0}^{\infty} t^{x-1} \mathrm{e}^{-t} \mathrm{~d} t
$$




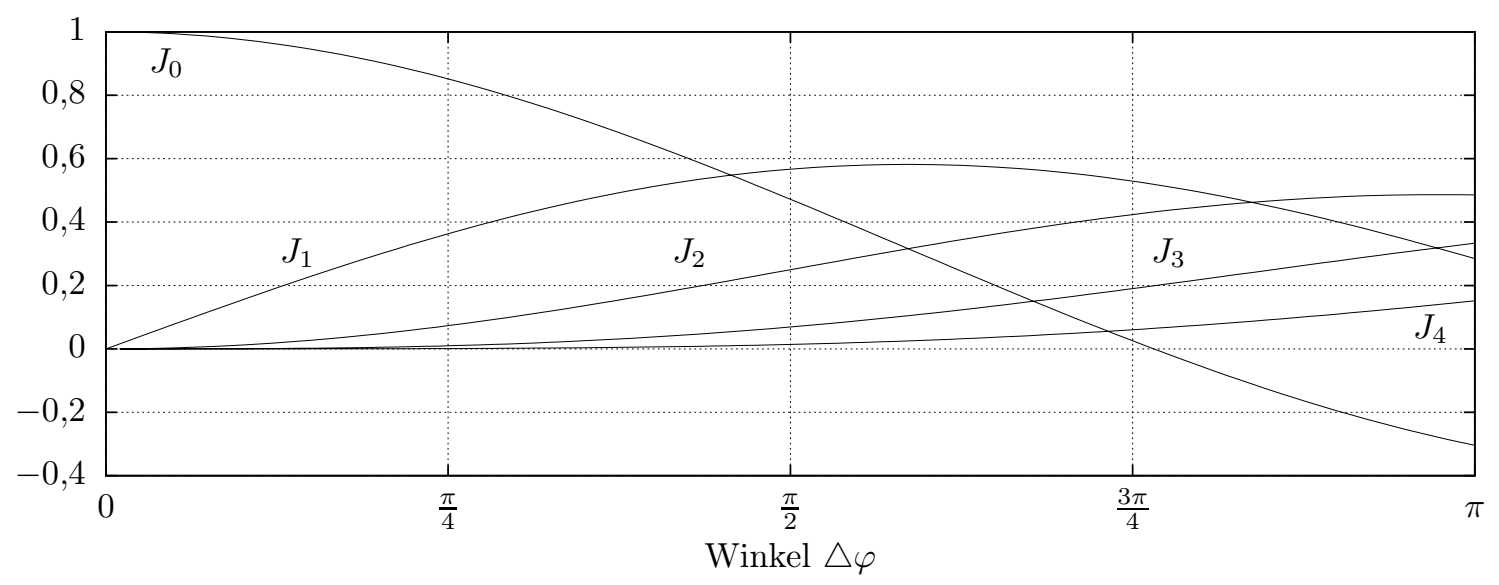

Bild 3.5: Bessel-Funktion erster Gattung bei $\nu=1$

in

$$
\begin{aligned}
\xi_{\mathrm{u} \nu}\left(x_{2}\right)= & J_{0}(\nu \Delta \varphi) \cdot \frac{2}{\nu} \sin \left(\frac{\nu \pi}{N_{2}}\right) \\
& +\sum_{n=1}^{\infty} J_{n}(\nu \Delta \varphi) \cdot\left[\cos \left(\frac{\nu \pi}{N_{2}}+n \tilde{b}_{2} x_{2}\right)-(-1)^{n} \cos \left(\frac{\nu \pi}{N_{2}}-n \tilde{b}_{2} x_{2}\right)\right] \\
= & J_{0}(\nu \Delta \varphi) \cdot \frac{2}{\nu} \sin \left(\frac{\nu \pi}{N_{2}}\right) \\
& +\frac{4}{\nu} \sum_{n=1,3, \ldots}^{\infty} J_{n}(\nu \Delta \varphi) \cdot \cos \left(\frac{\nu \pi}{N_{2}}\right) \cos \left(n \tilde{b}_{2} x_{2}\right) \\
& -\frac{4}{\nu} \sum_{n=2,4, \ldots}^{\infty} J_{n}(\nu \Delta \varphi) \cdot \sin \left(\frac{\nu \pi}{N_{2}}\right) \sin \left(n \tilde{b}_{2} x_{2}\right)
\end{aligned}
$$

umgeformt werden.

Damit liegt eine kontinuierliche Beschreibung des Einkopplungsfaktors über dem Winkel $x_{2}$ für eine sinusförmige Anordnung der Läufernuten vor. Hiermit kann die Spannungsdichte

$$
u_{\mathrm{i} \nu}^{\prime}\left(x_{2}, t\right)=\hat{u}_{\mathrm{i} \nu} \nu \frac{\xi_{\mathrm{u} \nu}\left(x_{2}\right)}{b_{\mathrm{z}}\left(x_{2}\right)} \cos \left(\nu x_{2}-\omega_{2 \nu} t+\varphi_{\nu}+\frac{\pi}{2}\right)
$$

berechnet werden, welche sich im kontinuierlichen Modell des Läuferkäfigs beim Übergang von einzelnen Läufermaschen in eine Art Maschendichte aus der induzierten Spannung $u_{\mathrm{i} \nu}$ entsprechend (3.57) ergibt. Aufgrund der Multiplikation von $\xi_{\mathrm{u} \nu}$ in (3.70) mit dem Kosinusterm enthält $u_{\mathrm{i} \nu}^{\prime}\left(x_{2}, t\right)$ dabei gerade Anteile mit den räumlichen Ordnungszahlen

$$
\nu_{\mathrm{u}}=\nu \pm n \tilde{b}_{2}, n \in \mathbb{Z}
$$




\subsubsection{Schrägung}

Die in der Masche $(n)$ induzierte Spannung wird bei geschrägter Maschine zu

$$
\begin{aligned}
u_{\mathrm{i}(n) \nu}(t) & =-\frac{\mathrm{d}}{\mathrm{d} t} \int_{-\frac{l_{\mathrm{i}}}{2}}^{\frac{l_{\mathrm{i}}}{2}} \int_{x_{2(n)}}^{x_{2(n+1)}} \hat{B}_{\nu} \cos \left(\nu\left(x_{2}+\frac{\gamma_{\mathrm{schr}}}{l_{\mathrm{i}}} z\right)-\omega_{2 \nu} t-\frac{\sigma}{l_{\mathrm{i}}} z+\varphi_{\nu}\right) r \mathrm{~d} x_{2} \mathrm{~d} z \\
& =\hat{u}_{\mathrm{i} \nu} \xi_{\mathrm{u}(n) \nu} \xi_{\mathrm{schr} \nu} \cos \left(\nu x_{2 \mathrm{z}(n)}-\omega_{2 \nu} t+\varphi_{\nu}-\frac{\pi}{2}\right) .
\end{aligned}
$$

Der in (3.73) enthaltene Schrägungsfaktor

$$
\xi_{\mathrm{schr} \nu}=\frac{\sin \left(\frac{\nu \gamma_{\mathrm{schr}}-\sigma}{2}\right)}{\frac{\nu \gamma_{\mathrm{schr}}-\sigma}{2}}
$$

ergibt sich dabei aus der in (3.72) enthaltenen Integration über $z$ und entspricht für $\sigma=0$ der überlicherweise in der Literatur verwendeten Variante [18].

Der Vollständigkeit halber sei an dieser Stelle angemerkt, dass schräglaufende Induktionswellen mit $\sigma \neq 0$ gegenüber geradelaufenden Induktionswellen mit $\sigma=0$ in der Ständerwicklung um den Ständerschrägungsfaktor

$$
\xi_{\mathrm{schr} 1 \nu}=\frac{\sin \left(\frac{\sigma}{2}\right)}{\frac{\sigma}{2}}
$$

niedrigere Spannungen induzieren. Der Unterschied zwischen (3.74) und (3.751) begründet sich dabei durch die Vereinfachung im vorliegenden Modell, dass die Ständernuten grundsätzlich als gerade betrachtet werden.

\subsubsection{Nutstreuinduktivitäten}

Die am Anfang von Abschnitt [3.6 eingeführte Stabstreuinduktivität $L_{\mathrm{s} \sigma}$ besteht im Wesentlichen aus der Selbstinduktivität infolge der Nutstreuung. Die Berechnung dieser Selbstinduktivität soll vereinfacht am Beispiel der in Bild 3.6] dargestellten Anordnung, bestehend aus einem Leiter in einer Nut, für den stromverdrängungsfreien Fall ähnlich dem Vorgehen in [19] beschrieben werden.

Da bei Variation der Läufernutpositionen eine gleichzeitige Variation der Läufernutgrößen beziehungsweise -geometrien naheliegend ist, soll die an dieser Stelle gezeigte Berechnung insbesondere den dadurch hervorgerufenen Einfluss auf die Nutstreuung aufzeigen.

Im Leitergebiet der Fläche $A$ fließst der felderregende Strom $i$, welcher bei vernachlässigbarer Stromverdrängung als im Leitergebiet gleichmäßig verteilte Stromdichte $\mathbf{S}$ angenommen werden kann.

Für die Nutbreite $b_{\mathrm{N}}^{*}(r)$ und die Breite des Leitergebiets $b_{\mathrm{L}}^{*}(r)$ ergibt sich damit bei ausschließlich parallel zur Winkelkoordinate $x_{2}$ gerichtetem magnetischen Fluss unter Anwendung des Durchflutungssatzes

$$
\int \mathbf{S} \mathrm{d} \mathbf{A}=\oint \mathbf{H} \mathrm{d} \mathbf{s}
$$




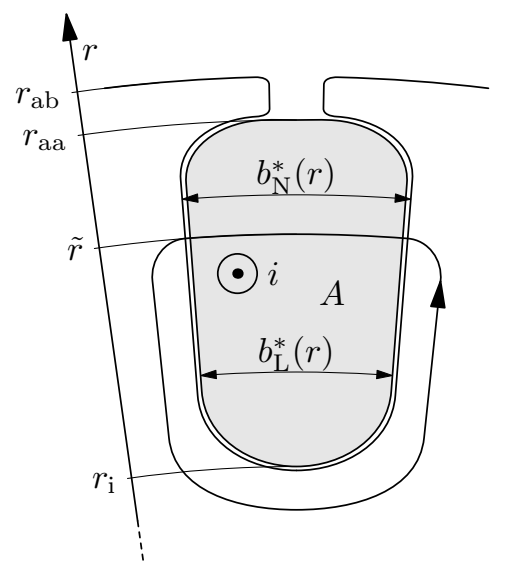

Bild 3.6: Ermittlung der Nutstreuinduktivität

die Stabstreuinduktivität

$$
L_{\mathrm{s} \sigma}=\frac{1}{i} \cdot \underbrace{l_{\mathrm{i}} \cdot(\int_{r_{\mathrm{i}}}^{r_{\mathrm{aa}}} \underbrace{\frac{\mu_{0}}{b_{\mathrm{N}}^{*}(\tilde{r})} \cdot \frac{i}{A} \cdot \int_{r_{\mathrm{i}}}^{\tilde{r}} b_{\mathrm{L}}^{*}(r) \mathrm{d} r}_{\text {Induktion } B(\tilde{r}) \text { im Leiterbereich }} r \tilde{r}+\int_{r_{\mathrm{aa}}}^{r_{\mathrm{ab}}} \frac{\mu_{0} \cdot i}{b_{\mathrm{N}}^{*}(r)} \mathrm{d} r)}_{=\Phi(i)} .
$$

Diese ist bei gleichbleibender Nutform, aber veränderter Nutbreite, also bei Skalierung um den Faktor $\vartheta \mathrm{zu} b_{\mathrm{N}}^{* \prime}(r)=\vartheta b_{\mathrm{N}}^{*}(r)$, proportional zu $1 / \bar{b}_{\mathrm{N}}^{*}$ beziehungsweise $1 / \vartheta$.

Aufgrund der eingeschränkten Gültigkeit von (3.77) ausschließlich für parallel zur Winkelkoordinate,$x_{2}$ " verlaufende magnetische Felder in der Nut, sind die damit für beliebige Nutformen berechneten Nutstreuinduktivitäten im Allgemeinen mit einem Modellbedingten Fehler behaftet. Folglich gilt auch die Proportionalität zu $1 / \vartheta$ nur eingeschränkt, lässt sich nurmerisch jedoch für die meißten Nutgeometrien näherungsweise bestätigen. Für die in Kapitel 7 gezeigten Beispiele ergibt sich aus der Einschränkung kein Fehler, da dort ausschließlich die nachfolgend beschriebene stromverdrängungsbehaftete Nutstreuinduktivität auf Basis numerischer Berechnungen verwendet ist.

\subsubsection{Einfluss der Stromverdrängung}

Im Läuferkäfig vieler Induktionsmaschinen hat die Stromverdrängung abhängig von der Nutgeometrie sowie der Ständerstromfrequenz $f_{1}$ und der Drehzahl $n$ einen nicht vernachlässigbaren Einfluss. Mit zunehmender Stabhöhe und zunehmender Frequenz drängt sich der Strom in erster Linie aufgrund einer durch Nutquerfelder selbst induzierten Spannung weiter an den Luftspalt, sodass der wirksame ohmsche Widerstand der Leiter steigt. Die Nutstreuinduktivität nimmt hingegen ab.

Soll die Geometrie der Läufernuten und somit auch der Stäbe gezielt variiert werden, so ist es notwendig, den Einfluss auf die Effekte der Stromverdrängung zu kennen oder zumindest abschätzen zu können.

Bei Hochstabläufern mit rechteckigem Querschnitt gemäß Bild 3.7a in Eisen sehr hoher Permeabilität $\left(\mu_{\mathrm{r}, \mathrm{Fe}} \rightarrow \infty\right)$ hat die Stromdichte $\mathbf{S}$ mit guter Näherung ausschließlich einen 


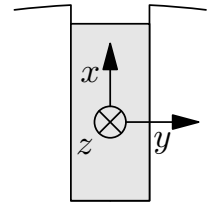

(a) Hochstab

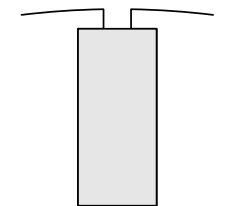

(b) Rechtecknut

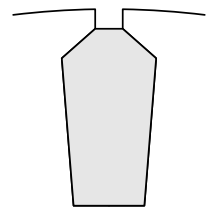

(c) Trapezform

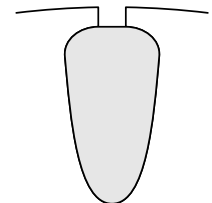

(d) Tropfenform

Bild 3.7: Verschiedene einfache Nutformen

Anteil in z-Richtung des in Bild $3.7 \mathrm{a}$ eingezeichneten kartesischen Koordinatensystems. Da die Feldlinien des vom Strom in der Nut erregten Magnetfelds senkrecht aus den Nutflanken austreten beziehungsweise in diese eintreten, hat die Induktion B bei Vernachlässigung von Randeffekten ausschließlich eine Komponente in $\mathbf{y}$-Richtung. Es gilt also $\mathbf{B}=B_{\mathrm{y}} \cdot \mathbf{e}_{\mathrm{y}}$. Folglich ist unter Vorgriff auf die nachfolgenden Überlegungen die Annahme konstanter Ströme über $y$ und $z$ zulässig. Damit lässt sich aus (3.77) der Zusammenhang

$$
B_{\mathrm{y}}(x)=\frac{b_{\mathrm{L}}}{b_{\mathrm{N}}} \cdot \mu_{0} \cdot \int_{r_{\mathrm{i}}}^{x} S_{\mathrm{z}}(x) \mathrm{d} \tilde{x}
$$

finden. Des Weiteren folgt aus dem Induktionsgesetz [15]

$$
\operatorname{rot} \mathbf{E}=-\frac{\mathrm{d} \mathbf{B}}{\mathrm{d} t}
$$

mit der induzierten elektrischen Feldstärke E die Beziehung

$$
\frac{\partial E_{\mathrm{z}}}{\partial x}=\frac{\mathrm{d} B_{\mathrm{y}}}{\mathrm{d} t}
$$

Weil dieses induzierte elektrische Feld $E_{\mathrm{z}}$ dem Stromfluss in der Nut in Abhängigkeit von der Koordinate $x$ unterschiedlich entgegen wirkt, tritt eine Stromverdrängung und damit eine Erhöhung des elektrischen Widerstands um den Faktor $k_{\mathrm{r}}$ in Erscheinung. Zudem wird die Stabstreuinduktivität um den Faktor $k_{\mathrm{l} \sigma}<1$ kleiner.

Da (3.78) und (3.80) nur vom Verhältnis der Leiterbreite $b_{\mathrm{L}}$ zur Nutbreite $b_{\mathrm{N}}$, nicht aber von der Nutbreite selbst abhängig sind, folgt, dass die stromverdrängungsbedingte Widerstands- und Streuinduktivitätserhöhung $k_{\mathrm{r}}$ und $k_{\mathrm{l} \sigma}$ bei Vernachlässigung von Randeffekten an der Nutöffnung ausschließlich von der Nuthöhe $h_{\mathrm{N}}$ und nicht von der Nutbreite $b_{\mathrm{N}}$ abhängig ist. Die Nutbreite kann also beliebig variiert werden, wobei sich sowohl der in Bild 3.4 dargestellte Stabwiderstand $R_{\mathrm{s}}$ als auch die Stabstreuinduktivität $L_{\sigma \mathrm{s}}$ umgekehrt proportional zur Nutbreite $b_{\mathrm{N}}$ ändern, solange sich $b_{\mathrm{N}}$ proportional zur Leiterbreite $b_{\mathrm{L}}$ verhält.

Die gezeigte analytische Beschreibung der Stromverdrängung lässt sich so einfach nur für Hochstabläufer gemäß Bild 3.7a finden. Die Effekte komplizierterer Geometrien sind vielmehr numerisch durch eine Finite-Elemente-Berechnung oder nicht so fein diskretisiert durch ein Teilleiterverfahren zu bestimmen.

Zur einfacheren Durchdringung der Abhängigkeit der Stromverdrängung von der Nutgeometrie soll im Folgenden die in Bild 3.8 dargestellte, relativ grob diskretisierte Anordnung 


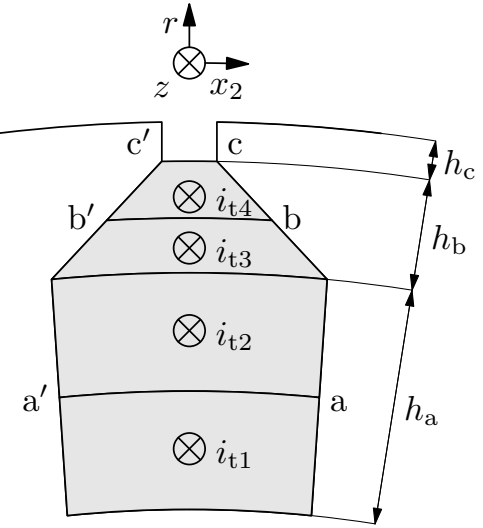

(a) Ersatzanordnung eines massiven Leiters in einer Nut

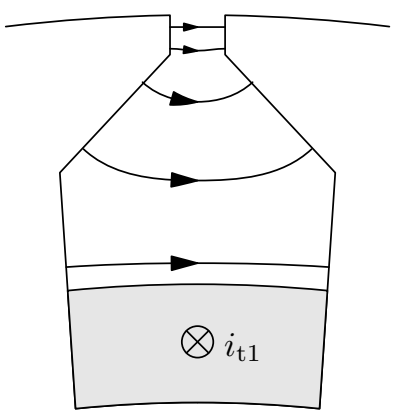

(b) Feld von Teilleiter ,1““

Bild 3.8: Modell zur Abschätzung der Stromverdrängung

betrachtet werden. Der Leiter in der Nut ist dabei durch vier Teilleiter ersetzt, die entweder realitätsnäher als Leitergebiete oder abstrakter als Linienleiter in $\mathbf{z}$-Richtung an den mit der elektrischen Durchflutung gekennzeichneten Stellen betrachtet werden können.

In der in Bild 3.8a dargestellten Nut trägt jeder Teilleiter einen Anteil zum magnetischen Feld in derselben Nut bei. Beispielhaft ist das Feld des Teilleiters „1" in Form von Feldlinien in der Nut in Bild 3.8b skizziert.

Modellbildend wird angenommen, dass die Teilleiter an den Stirnflächen der Maschine kurzgeschlossen sind. Die jeweils übereinanderliegenden Teilleiter bilden in diesem Fall Leiterschleifen, welche beginnend am Nutgrund mit den Indizes $s=1,2,3, \ldots$ bezeichnet werden sollen.

Unter Vernachlässigung der Randeffekte an den Stirnseiten der Maschine werden die Leiterschleifen von den Flüssen $\boldsymbol{\Phi}_{s}(t)$ mit Komponenten in $\mathbf{x}$ - und $\mathbf{r}$-Richtung durchsetzt. Diese Flüsse führen in den Leiterschleifen zu induzierten Spannungen, welche Ströme treiben, was als Stromverdrängung in Erscheinung tritt. Eine Vereinfachung ergibt sich bei der Darstellung der Teilleiter durch Linienleiter in der Mitte einer symmetrischen Nut, womit die Flüsse $\boldsymbol{\Phi}_{s}(t)$ ausschließlich eine $\mathbf{x}$-Komponente besitzen. Es ergibt sich damit das Ersatzschaltbild eines Leiters gemäßs Bild 3.9.

Mit den Kopplungsfaktoren $k_{t s}$, die den Einfluss des Stroms $\underline{i}_{t t}$ im Teilleiter $t$ auf den Fluss $\Phi_{s}$ in Leiterschleife $s$ angeben, folgt

$$
\Phi_{s}(t)=\sum_{t=1}^{4} k_{t s} \cdot i_{\mathrm{t} t}(t) \quad \text { mit } \quad i_{\mathrm{t} t}(t)=\Re\left\{\underline{i}_{\mathrm{t} t}(t)\right\}=\Re\left\{\hat{i}_{\mathrm{t} t} \cdot e^{\mathrm{j} \omega t}\right\} .
$$

Dabei gilt für die in Bild 3.8 dargestellte Anordnung

$$
k_{11}, k_{12}, k_{13}, k_{22}, k_{23}, k_{33}>0 \quad \text { und } \quad k_{21}, k_{31}, k_{32}, k_{41}, k_{42}, k_{43}<0
$$

wobei die zweitgenannte Gruppe, also die Kopplungsfaktoren, welche Flussanteile repräsentieren, die gegenüber dem jeweiligen erregenden Teilleiter näher am Nutgrund verlaufen, bei Annahme von $\mu_{\mathrm{Fe}} \rightarrow \infty$ weitgehend vernachlässigbar ist. 


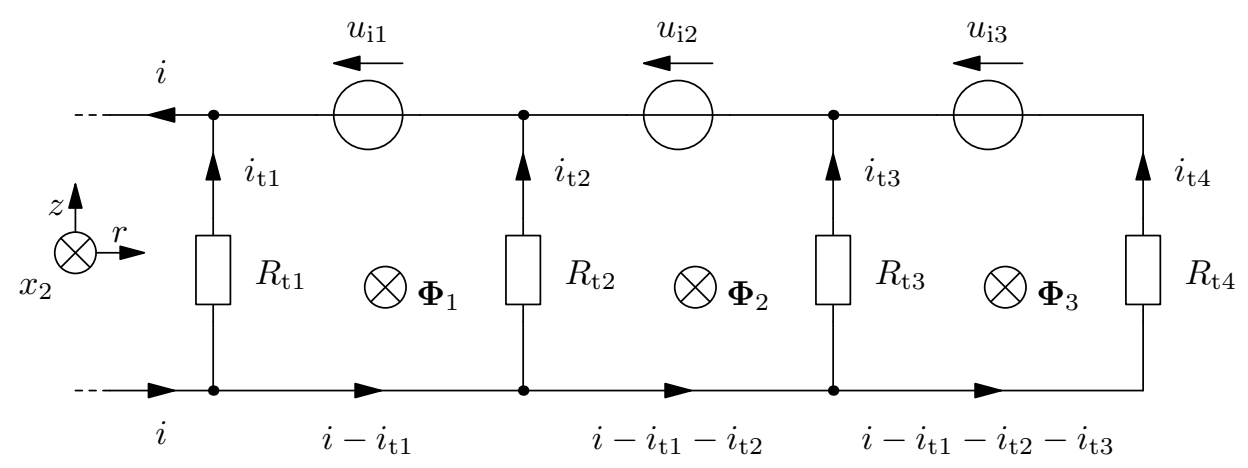

Bild 3.9: Ersatzschaltbild eines Leiters

Die Systemgleichung des in Bild 3.9 dargestellten Ersatzschaltbilds ergibt sich zu

$$
\underbrace{\left(\begin{array}{ccc}
\frac{R_{\mathrm{t} 1}}{\mathrm{j} \omega} \underbrace{\left(k_{11}-k_{41}\right)}_{>0} & \frac{-R_{\mathrm{t} 2}}{\mathrm{j} \omega}+\left(k_{21}-k_{41}\right) & \left(k_{31}-k_{41}\right) \\
\underbrace{\left(k_{12}-k_{42}\right)}_{>0} & \frac{R_{\mathrm{t} 2}}{\mathrm{j} \omega}+\underbrace{\left(k_{22}-k_{42}\right)}_{>0} & \frac{-R_{\mathrm{t} 3}}{\mathrm{j} \omega}+\left(k_{32}-k_{42}\right) \\
\frac{R_{\mathrm{t} 4}}{\mathrm{j} \omega} \underbrace{\left(k_{13}-k_{43}\right)}_{\mathbf{A}} & \frac{R_{\mathrm{t} 4}}{\mathrm{j} \omega}+\underbrace{\left(k_{23}-k_{43}\right)}_{>0} & \frac{R_{\mathrm{t} 3}+R_{\mathrm{t} 4}}{\mathrm{j} \omega}+\underbrace{\left(k_{33}-k_{43}\right)}_{>0}
\end{array}\right)}_{>0} \cdot \underline{\mathbf{i}}_{\mathrm{t}}=\mathbf{b}
$$

mit

$$
\underline{\mathbf{i}}_{\mathrm{t}}=\left(\begin{array}{c}
\underline{i}_{\mathrm{t} 1} \\
\underline{i}_{\mathrm{t} 2} \\
\underline{i}_{\mathrm{t} 3}
\end{array}\right) \quad \text { und } \quad \mathbf{b}=\left(\begin{array}{c}
-k_{41} \cdot i(t) \\
-k_{42} \cdot i(t) \\
-k_{43} \cdot i(t)+\frac{R_{\mathrm{t} 4}}{\mathrm{j} \omega} \cdot i(t)
\end{array}\right)
$$

Mit (3.83) und (3.84) lässt sich nun der Einfluss der einzelnen Kopplungen verstehen.

Unter der bereits genannten Vernachlässigung von $k_{21}, k_{31}, k_{32}, k_{41}, k_{42}$ und $k_{43}$, wird $\mathbf{A}$ zu einer unteren Dreiecksmatrix mit ausschließlich positiven Elementen. Folglich ergeben sich die Stromdichten, also die Ströme der Teilleiter $i_{\mathrm{t} 1}$ bis $i_{\mathrm{t} 4}$ bezogen auf die Teilleiterflächen, bei $\omega>0$ vom Betrag abnehmend von der Nutöffnung zum Nutgrund. Da bei einem Hochstabläufer gemäß Bild 3.7 a die Fläche der Nut und somit auch des leitfähigen Gebiets proportional zur Nutbreite sind, ist der Widerstand genau wie die Kopplungsfaktoren umgekehrt proportional zu dieser Breite. A und $\mathbf{b}$ sind damit im gleichen Maße von der Nutbreite abhängig, sodass das Gleichungssystem (3.83) unabhängig von der Nutbreite $b_{\mathrm{N}}$ ist. Die Stromverdrängungskoeffizienten $k_{\mathrm{r}}$ und $k_{\mathrm{l} \sigma}$ eines Hochstabläufers sind also, wie bereits weiter oben erwähnt, unabhängig von der Nutbreite.

Komplizierter werden die Zusammenhänge für die anderen in Bild [3.7 dargestellten Nutformen. Mit zunehmender Breite der Nut verändern sich bei Rechtecknuten entsprechend Bild 3.7b die Feldanteile durch die parallelen Flanken, welche entsprechend Bild 3.8 als ,a" und „c" bezeichnet werden, genau wie beim Hochstabläufer. Folglich hat dieser Feldanteil keine Auswirkung in Form einer Änderung der Stromverdrängung. Anders verhält es sich 
mit dem Anteil durch die waagerechten Flanken „,““. Diese Flanken rücken, genau wie die anderen Flanken, mit zunehmender Nutbreite weiter auseinander. Daher tritt auch hier eine Abnahme der Induktion ein. Da die Flanken selbst jedoch breiter werden, verhält sich der Fluss nicht streng umgekehrt proportional zur Nutbreite, sodass die Flanken „,b“ im Verhältnis zu den Anderen bei zunehmender Nutbreite durch mehr Fluss durchsetzt werden. Folglich werden sämtliche Kopplungsfaktoren $k_{t s}$ mit zunehmender Nutbreite relativ zu den Widerständen größer. Damit ergibt sich bei Nutverbreiterung eine Vergrößerung der Widerstandskoeffizienten $k_{\mathrm{r}}$. Da gleichzeitig der Einfluss der bei Stromverdrängung im Wesentlichen elektrisch durchfluteten Leiterbereiche auf die Nutstreuinduktivitäten steigt, werden die Streuinduktivitätskoeffizienten $k_{1 \sigma}$, wie in Bild 3.10 ersichtlich, nicht kleiner, sondern größer, sodass sich diese an den Wert „1“ annähern. Der Einfluss der Frequenz des Läuferstroms auf die Nutstreuinduktivitäten sinkt folglich mit zunehmender Nutbreite während der Einfluss auf die Stabwiderstände steigt.

Eine weitere Zunahme der Komplexität ergibt sich mit einer aus Trapezen zusammengesetzten Nut gemäß Bild [3.7c Wird die Nut in ihrer Breite skaliert und die Höhe $h_{\mathrm{b}}$ konstant gehalten, so ändert sich sowohl die Länge, als auch der Winkel der Flanke „,““. Der Einfluss der Winkeländerung von eher senkrechter Lage auf eher waagerechte Lage bei Verbreiterung der Nut führt zu tiefer in der Nut verlaufenden Flusskomponenten und die Verlängerung zu relativ gesehen größeren Komponenten, sodass die Stromverdrängung verstärkt wird.

Ähnlich der Trapezform gemäß Bild $3.7 \mathrm{c}$ verhalten sich auch die in Bild $3.7 \mathrm{~d}$ gezeigten tropfenförmigen Nuten. Aufgrund der Ähnlichkeit lässt sich dies bereits aus der Betrachtung der Nutformen vermuten.

In Abschnitt 7.3.1 wird gezeigt, dass es sinnvoll ist, wenn die Stromverdrängungskoeffizienten $k_{\mathrm{r}}$ und $k_{\mathrm{l} \sigma}$ unabhängig von der Nutbreite sind. Bei üblichen Nutformen - ausgenommen Hochstäben - ist dies jedoch nicht der Fall. Es ist daher wünschenswert, die Änderung der Koeffizienten durch weitere Maßnahmen auszugleichen. Naheliegend ist dabei zum Beispiel die Änderung der Höhe $h_{\mathrm{b}}$ gemäß Bild 3.8. Um die Nutstreuung nicht zu stark zu beeinflussen, muss $h_{\mathrm{a}}$ dabei nahezu konstant gehalten werden. Eine Verkleinerung von $h_{\mathrm{b}}$ verändert den Winkel der Flanke „b“ derart, dass ein stärkerer Einfluss auf die Stromverdrängung zu erwarten wäre. Da die Flanke jedoch auch kürzer wird und die Breite der Nut erst weiter in der Nähe der Nutöffnung schmaler wird, werden die Kopplungskoeffizienten und somit auch die Stromverdrängung kleiner. Es wäre also möglich, die Änderung der Stromverdrängungskoeffizienten aufgrund einer größeren Nutbreite durch eine Verkleinerung von $h_{\mathrm{b}}$ zu kompensieren. Dieser Ansatz disqualifiziert sich jedoch dadurch, dass der für den Fluss im Zahn zur Verfügung stehende Platz im Zahnkopf kleiner wird, obwohl der Fluss größer wird.

Mit der hier aufgeführten qualitativen Betrachtung der Veränderung der Stromverdrängungskoeffizienten $k_{\mathrm{r}}$ und $k_{\mathrm{l} \sigma}$ in Abhängigkeit von verschiedenen Geometrieänderungen der Nuten wird ersichtlich, dass die Forderung nach breitenunabhängigen Koeffizienten bei den gängigen Nutformen - ausgenommen den Hochstäben - nicht erfüllbar ist.

Da sich die geschlossene analytische Berechnung der Stromverdrängungseffekte, wie gezeigt, schwierig gestaltet, scheidet dieses Vorgehen für die praktische Berechnung der meisten Geometrien aus. In vielen analytischen Programmen wird daher ein Teilleiterverfahren angewandt, bei dem jeder Leiter, ähnlich wie bei der in diesem Abschnitt aufgeführten 


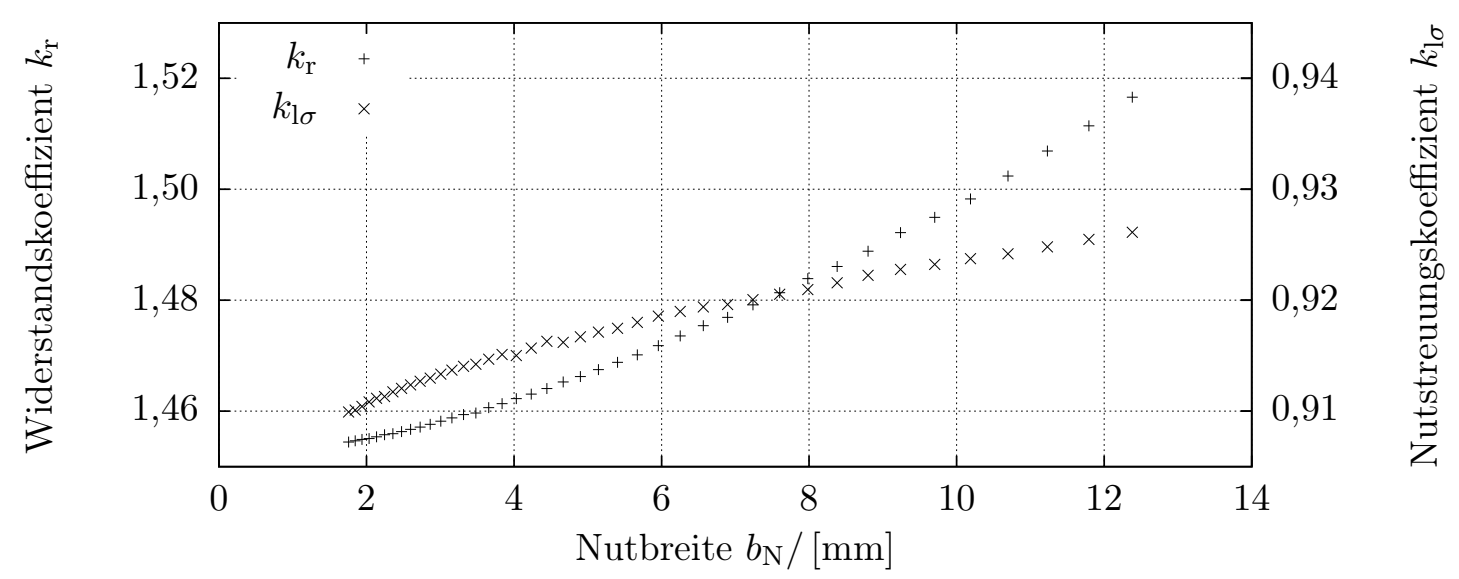

Bild 3.10: Einfluss der Nutbreite auf die Stromverdrängungskoeffizienten der in Bild 7.3a dargestellten Beispielmaschine „B“ bei der Frequenz des Läuferstroms $f_{2}=50 \mathrm{~Hz}$

Herleitung, in mehrere Teilleiter zerlegt wird, die als an den Stirnseiten kurzgeschlossen angesehen werden. Da dieses Verfahren einer grob diskretisierten Feldberechnung per Finite-Elemente-Methode (FEM) ähnlich ist, wurde bei den Berechnungen in Kapitel[7 direkt auf per FEM numerisch berechnete Kennlinienfelder für die einzelnen Nutgeometrien zurückgegriffen.

Um einen quantitativen Eindruck des Einflusses der Nutbreite auf die Stromverdrängungskoeffizienten $k_{\mathrm{r}}$ und $k_{\mathrm{l} \sigma}$ zu geben, sind diese beispielhaft für die in Bild [7.3 auf Seite 87 dargestellte Maschine „, $\mathrm{B}$ “ in Bild [3.10] aufgezeigt.

\subsubsection{Diskrete Betrachtung des Läuferkäfigs}

Zur diskreten Betrachtung der Vorgänge im Käfig wird dieser entsprechend dem Ersatzschaltbild aus Bild 3.4 modelliert.

Aus den vorhergehenden Abschnitten dieses Kapitels können einige der verwendeten Ersatzschaltbildelemente als bekannt angenommen werden. Dies sind insbesondere die induzierten Spannungen aufgrund der Ständerinduktionswellen $u_{\mathrm{i} 1(n)}$ gemäß Abschnitt 3.6.1 und die Stabstreuinduktivitäten $L_{\mathrm{s} \sigma(n)}$ gemäß der Abschnitte 3.6.2 und 3.6.3, Bei bekannter Geometrie und Leitfähigkeit des Stabmaterials sowie der Berücksichtigung der in 3.6.3 beispielhaft gezeigten Stromverdrängung gilt dies ebenfalls für die Stabwiderstände $R_{\mathrm{s}(n)}$.

Von der Herleitung der Berechnung der Widerstände und Streuinduktivitäten der Ringsegmente $R_{\mathrm{r}(n)}$ sowie $L_{\mathrm{r} \sigma(n)}$ soll an dieser Stelle abgesehen werden. Das in Kapitel 7 erwähnte und im Rahmen dieser Arbeit entwickelte Rechenprogramm verwendet hierfür vom Benutzer vorgegebene Werte, welche bei Variation der Nutpositionen linear interpoliert werden.

Die somit verbleibenden unbekannten Ersatzschaltbildelemente sind die Luftspaltfeldselbstinduktivitäten der Käfigmaschen $L_{\delta(n)}$ sowie die Gegeninduktivitäten $M_{n k}$ zwischen den einzelnen Maschen, welche in Bild 3.4 vereinfachend durch die daraus resultierenden induzierten Spannungen $u_{\mathrm{M}(n)}$ repräsentiert werden.

Beide im letzten Absatz genannten Induktivitäten sind abhängig vom läuferseitig erregten 
Luftspaltfeld. Dieses lässt sich, wie für das ständerseitig erregte Luftspaltfeld bereits in Abschnitt 3.5 gezeigt, aus der Felderregung $V_{2}\left(x_{2}, t\right)$ und den Luftspaltleitwerten $\lambda\left(x_{2}, \rho\right)$ berechnen. Dabei ist die Felderregung gerade, wie in Abschnitt 3.3 gezeigt, abhängig vom Integral des Strombelags über den Umfang des Läufers, beziehungsweise bei Annahme der Stäbe als Linienleiter von den Ringströmen $i_{\mathrm{r}(n)}(t)$, die aufgrund der Beziehung

$$
i_{\mathrm{r}(n+1)}=i_{\mathrm{r}(n)}+i_{\mathrm{s}(n)}
$$

einer Integration des Strombelags über den Umfang des Läufers entsprechen. Es wird damit ersichtlich, dass die Wahl des Vektors der Ringströme $\mathbf{i}_{\mathrm{r}}$ als Zustandsgröße des Läufernetzwerks vorteilhaft ist.

Abhängig von den Ringströmen wird die von jedem Zahn $(n)$ ausgehende Felderregung damit zu

$$
V_{2(n)}(t)=i_{\mathrm{r}(n)}(t)+c(t)
$$

Die Integrationskonstante $c(t)$ bestimmt sich dabei, wie bereits in Abschnitt 3.5 gezeigt, aus der Bedingung

$$
\int_{-l_{\mathrm{i}} / 2}^{l_{\mathrm{i}} / 2} \int_{0}^{2 \pi} B(x, t, z) r \mathrm{~d} x \mathrm{~d} z=\Phi(t) \stackrel{!}{=} 0
$$

welche sich mit den Zahnflüssen $\Phi_{(n)}$ in

$$
\sum_{n} \underbrace{\int_{-l_{\mathrm{i}} / 2}^{l_{\mathrm{i}} / 2} \int_{x_{(\mathrm{n})}}^{x_{(\mathrm{n}+1)}}\left[i_{\mathrm{r}(n)}(t)+c(t)\right] \cdot \lambda\left(x_{2}, \rho, z\right) \mathrm{d} x_{2} \mathrm{~d} z \stackrel{!}{=} 0}_{=\frac{1}{r} \cdot \Phi_{(n)}\left(\rho, i_{\mathrm{r}(n)}(t)\right)}
$$

überführen lässt. Daraus ergibt sich die Integrationskonstante

$$
c(t)=\frac{-\sum_{n} \int_{-l_{\mathrm{i}} / 2}^{l_{\mathrm{i}} / 2} \int_{x_{(\mathrm{n})}}^{x_{(\mathrm{n}+1)}} i_{\mathrm{r}(n)}(t) \cdot \lambda\left(x_{2}, \rho, z\right) \mathrm{d} x_{2} \mathrm{~d} z}{2 \pi \cdot \int_{-l_{\mathrm{i}} / 2}^{l_{\mathrm{i}} / 2} \lambda_{0 \mu}(\rho, z) \mathrm{d} z}
$$

mit $\rho=\Omega \cdot t+\rho_{0}$, wobei $\lambda_{0 \mu}(\rho, z)$ entsprechend (3.40) der über $x_{2}$ invariante Anteil des Luftspaltleitwerts $\lambda_{\nu \mu}\left(x_{2}, \rho, z\right)$ ist.

Aus den somit bekannten Zahnflüssen lassen sich die Selbst- und Gegeninduktivitäten der einzelnen Käfigmaschen, welche im Allgemeinen vom Drehwinkel $\rho$ abhängen, also bei $\Omega \neq 0$ zeitvariant sind, mit

bzw.

$$
\begin{array}{r}
L_{\delta(n)}(\rho)=\frac{\Phi_{(n)}\left(\rho, i_{\mathrm{r}(n)}\right)}{i_{\mathrm{r}(n)}} \\
M_{n k}(\rho)=M_{k n}(\rho)=\frac{\Phi_{(k)}\left(\rho, i_{\mathrm{r}(n)}\right)}{i_{\mathrm{r}(n)}}
\end{array}
$$


$\mathrm{Zu}$

$$
L_{\delta(n)}(\rho)=\sum_{\nu, \mu, \tilde{\nu}, \tilde{\mu}, \breve{\mu}}\left[r l_{\mathrm{i}} \cdot\left(1-\frac{\xi_{\mathrm{schr} \mu} \int_{x_{2(n)}}^{x_{2(n+1)}} \lambda_{\nu \mu}\left(x_{2}, \rho\right) \mathrm{d} x_{2}}{2 \pi \cdot \xi_{\mathrm{schr} \breve{\mu}} \cdot \lambda_{0 \breve{\mu}}(\rho)}\right) \cdot \xi_{\mathrm{schr} \tilde{\mu}} \int_{x_{2(n)}}^{x_{2(n+1)}} \lambda_{\tilde{\nu} \tilde{\mu}}\left(x_{2}, \rho\right) \mathrm{d} x_{2}\right]
$$

sowie

$$
M_{n k}(\rho)=-\sum_{\nu, \mu, \tilde{\nu}, \tilde{\mu}, \breve{\mu}}\left[r l_{\mathrm{i}} \xi_{\mathrm{schr} \mu} \xi_{\mathrm{schr} \tilde{\mu}} \cdot \frac{\int_{x_{2(n)}}^{x_{2(n+1)}} \lambda_{\nu \mu}\left(x_{2}, \rho\right) \mathrm{d} x_{2}}{2 \pi \cdot \xi_{\mathrm{schr} \breve{\mu}} \cdot \lambda_{0 \breve{\mu}}(\rho)} \cdot \int_{x_{2(k)}}^{x_{2(k+1)}} \lambda_{\tilde{\nu} \tilde{\mu}}\left(x_{2}, \rho\right) \mathrm{d} x_{2}\right]
$$

bestimmen.

Da in (3.92) und (3.93), wie bereits bei der Reihenschaltung der Luftspaltleitwertwellen von Ständer und Läufer in (3.39), eine Fourierreihe im Nenner steht, lässt sich auch hier auf einfache Weise keine wiederum als Fourierreihe über $\rho$ dargestellte Form der Induktivitäten finden. Zur Lösung dieses Problems bietet sich eine Fouriertransformation über für verschiedene Läuferdrehwinkel $\rho$ berechnete Induktivitäten an. Alternativ können die Fourierreihen im Nenner von (3.92) und (3.93), ähnlich wie in Abschnitt 3.4 für die Reihenschaltung der Luftspaltleitwertverteilungen gezeigt, durch das konstante Glied, also

$$
\lambda_{0 \breve{\mu}}(\rho) \approx \lambda_{0}
$$

angenähert werden. Dabei beschränken sich die Fourierreihe im Nenner von (3.92) und (3.93) gegenüber der bei Reihenschaltung der Luftspaltleitwerte auf die nicht von der Winkelkoordinate $x_{2}$ abhängigen Leitwertwellen. Folglich lässt sich hier ein kleinerer Fehler als bei der in Abschnitt 3.4 gezeigten und in Abschnitt 5.1 untersuchten Reihenschaltung erwarten. Damit erscheint die Anwendung von (3.94) für viele Fälle legitim.

Es ergeben sich letztendlich Induktivitäten der Form

bzw.

$$
\begin{array}{r}
L_{\delta(n)}(\rho)=\sum_{\kappa} L_{\delta(n) \kappa} \cos \left(\kappa \rho+\varphi_{\kappa}\right) \\
M_{n k}(\rho)=M_{k n}(\rho)=\sum_{\kappa} M_{n k \kappa} \cos \left(\kappa \rho+\varphi_{\kappa}\right),
\end{array}
$$

wobei für $M_{n k}(\rho)$ und $M_{k n}(\rho)$ die zum Beispiel in [35] hergeleitete Gleichheit angenommen wurde.

Um die in $L_{\delta(n)}(\rho)$ und $M_{n k}(\rho)$ enthaltenen Ordnungszahlen und somit auch die Frequenzen zu identifizieren, sei die Näherung gemäß (3.94) angenommen. Aus der Aufsummierung über alle $\nu, \mu, \tilde{\nu}$ und $\tilde{\mu}$ in (3.92) und (3.93) ergeben sich damit alle Ordnungszahlen $\kappa$ mit

$$
\kappa=(\mu-\nu) \pm(\tilde{\mu}-\tilde{\nu})
$$


wobei sich die Differenzen $(\mu-\nu)$ beziehungsweise $(\tilde{\mu}-\tilde{\nu})$ aus der Transformation der gemäß (3.40) dargestellten Luftspaltleitwertwellen in das läuferfeste Koordinatensystem ergeben. Die Kreisfrequenzen der Induktivitäten im läuferfesten Koordinatensystem werden damit $\mathrm{zu}$

$$
\omega_{2 \kappa}=\kappa \Omega
$$

Damit sind alle Elemente des Ersatzschaltbilds aus Bild 3.4 bekannt, und folglich kann das elektrische Netzwerk des Läuferkäfigs berechnet werden.

Für jede einzelne Kreisfrequenz $\omega_{2 \nu}$ der in den Maschen induzierten Spannungen ergibt sich bei Vernachlässigung der Zeitvarianz der Induktivitäten, also unter Verwendung der zeitlichen Mittelwerte $\bar{L}_{\delta(n)}$ und $\bar{M}_{n k}$, das aus den $N_{2}$ Gleichungen

$$
\begin{aligned}
\underline{u}_{\mathrm{i} 1 \nu(n)}= & \left(R_{\mathrm{s}(n)}+R_{\mathrm{s}(n+1)}+2 R_{\mathrm{r}(n)}\right) \underline{i}_{\mathrm{r} \nu(n)} \\
& -\left(R_{\mathrm{s}(n)}+\mathrm{j} \omega_{2 \nu} L_{\mathrm{s} \sigma(n)}\right) \underline{i}_{\mathrm{r} \nu(n-1)}-\left(R_{\mathrm{s}(n+1)}+\mathrm{j} \omega_{2 \nu} L_{\mathrm{s} \sigma(n+1)}\right) \underline{i}_{\mathrm{r} \nu(n+1)} \\
& +\mathrm{j} \omega_{2 \nu}\left(L_{\mathrm{s} \sigma(n)}+L_{\mathrm{s} \sigma(n+1)}+2 L_{\mathrm{r} \sigma \nu(n)}+\bar{L}_{\delta(n)}\right) \underline{i}_{\mathrm{r} \nu(n)}+\mathrm{j} \omega_{2 \nu} \sum_{k=1 ; k \neq n}^{N_{2}} \bar{M}_{n k} \underline{i}_{\mathrm{r} \nu(k)}
\end{aligned}
$$

mit $n=1,2, \ldots N_{2}$ bestehende Gleichungssystem.

Gemäß (3.59) ergeben sich für die direkt vom Ständerstrom herrührenden induzierten Spannungen im Läufer ausschließlich Kreisfrequenzen, welche aus einer Kombination der positiven oder negativen Ständerkreisfrequenz $\omega_{1}$ und einem Vielfachen der Winkelgeschwindigkeit des Läufers $\Omega$ bestehen. Auch bei Berücksichtigung der Zeitvarianz der Induktivitäten mit den Kreisfrequenzen gemäß (3.98) ergeben sich damit im Läufer folglich ausschließlich die Frequenzen

$$
\omega_{2 \nu}= \pm \omega_{1}+k \cdot \Omega \text { mit } k \in Z
$$

Es ist damit möglich, das durch (3.99) definierte Gleichungssystem mit $N_{2}$ Gleichungen und $N_{2}$ Unbekannten in ein Gleichungssystem mit $n_{\kappa} \cdot N_{2}$ Gleichungen und eben so vielen Unbekannten bei Berücksichtigung von $n_{\kappa}$ diskreten Kreisfrequenzen gemäß (3.100) zu überführen.

Da ein derartiges Gleichungssystem zu einem beachtlichen Rechenaufwand führt, bietet sich als alternatives Lösungsverfahren eine Iteration aus frequenzentkoppelten Berechnungen der $\kappa$ Gleichungssysteme an. Die Kopplung der Gleichungssysteme wird berücksichtigt, indem, wie auch in $[23,26]$ durchgeführt, nach jedem Iterationsschritt die in den Gleichungssystemen anderer Kreisfrequenz induzierten Spannungen berechnet werden.

\subsubsection{Kontinuierliche Betrachtung des Läuferkäfigs}

Um eine gegenüber dem im vorhergehenden Abschnitt aufgezeigten Berechnungsverfahren kompaktere Darstellung der Vorgänge im Läufer zu erlauben, soll eine kontinuierliche Beschreibung mit unendlich vielen Käfigstäben entwickelt werden. Somit wird es möglich, 
direkt mit den als Fourierreihen dargestellten Variablen, das heißt zum Beispiel Induktionen, Widerständen oder Strömen, zu rechnen. Da es sich bei der kontinuierlichen Darstellung um eine abstrakte Näherung des realen Läufers handelt, sind gegenüber der diskreten Betrachtung weniger exakte, aber einfacher durchdringbare Formeln zu erwarten.

Es sei angemerkt, dass es nicht das Ziel einer derartigen kontinuierlichen Betrachtung sein kann, eine einfachere Berechnungsmöglichkeit für das komplette Luftspaltspektrum von Induktionsmaschinen darzustellen. Dies lässt sich dadurch begründen, dass aus dem Gleichungssystem mit $N_{2}$ unbekannten Strömen je Frequenz, wie es in Abschnitt 3.6.4 zu finden ist, ein deutlich aufwändigeres Gleichungssystem mit theoretisch unendlich vielen Fourierkoeffizienten je Frequenz wird.

\subsubsection{Betrachtung ohne Berücksichtigung der realen Läuferzahnbreiten}

Die Idee der kontinuierlichen Betrachtung des Läufers ist die Darstellung des Käfigs durch unendlich viele dicht gepackte Stäbe, die in eine Art Flächenleiter übergehen, welcher einen Stromfluss ausschließlich entlang der $z$-Achse, beziehungsweise bei Schrägung mit entsprechendem Winkel, zulässt. Aus den diskreten Ringströmen $i_{\mathrm{r}(n)}(t)$ wird somit eine kontinuierliche Stromverteilung im Kurzschlussring $i_{\mathrm{r}}\left(x_{2}, t\right)$ und aus den diskreten Stabströmen $i_{\mathrm{s}(n)}(t)$ eine Stabstromdichte $i_{\mathrm{s}}^{\prime}\left(x_{2}, t\right)$ über dem Winkel $x_{2}$ mit

$$
i_{\mathrm{s}}^{\prime}\left(x_{2}, t\right)=\frac{\mathrm{d}}{\mathrm{d} x_{2}} i_{\mathrm{r}}\left(x_{2}, t\right) .
$$

Daraus lässt sich analog zu den Ausführungen in Abschnitt 3.3 über den Strombelag die Felderregung des Läufers zu

$$
V_{2}\left(x_{2}, t\right)=\int \underbrace{A_{2}\left(x_{2}, t\right)}_{=i_{\mathrm{s}}^{\prime}\left(x_{2}, t\right)} \mathrm{d} x_{2}=\int \frac{\mathrm{d}}{\mathrm{d} x_{2}} i_{\mathrm{r}}\left(x_{2}, t\right) \mathrm{d} x_{2}=i_{\mathrm{r}}\left(x_{2}, t\right)+c(t)
$$

bestimmen. Die vom Läufer erregte Luftspaltinduktion ergibt sich damit zu

$$
B_{2}\left(x_{2}, t\right)=\lambda\left(x_{2}, t\right) i_{\mathrm{r}}\left(x_{2}, t\right)+\lambda\left(x_{2}, t\right) c(t) .
$$

$\mathrm{Zu}$ beachten ist, dass (3.102) und (3.103) für einen Läufer mit unendlich vielen infinitesimal schmalen Nuten entwickelt sind. Eine Anpassung an endliche Zahnbreiten wird weiter unten - zusammen mit (3.112) - eingeführt.

Ist die Maschine hinreichend symmetrisch aufgebaut, beziehungsweise ist der magnetische Leitwert über die Stirnflächen der Maschine hinreichend klein, so ergibt sich der Mittelwert der Luftspaltinduktion zu Null, sodass die Integrationskonstante $c(t)$ bestimmt werden kann. Es gilt

$$
\begin{gathered}
\int_{0}^{2 \pi} B_{2}\left(x_{2}, t\right) \mathrm{d} x_{2}=0 \\
\Leftrightarrow \int_{0}^{2 \pi} \lambda\left(x_{2}, \rho\right) i_{\mathrm{r}}\left(x_{2}, t\right) \mathrm{d} x_{2}=-\int_{0}^{2 \pi} \lambda\left(x_{2}, \rho\right) c(t) \mathrm{d} x_{2}=-c(t) \int_{0}^{2 \pi} \lambda\left(x_{2}, \rho\right) \mathrm{d} x_{2}
\end{gathered}
$$




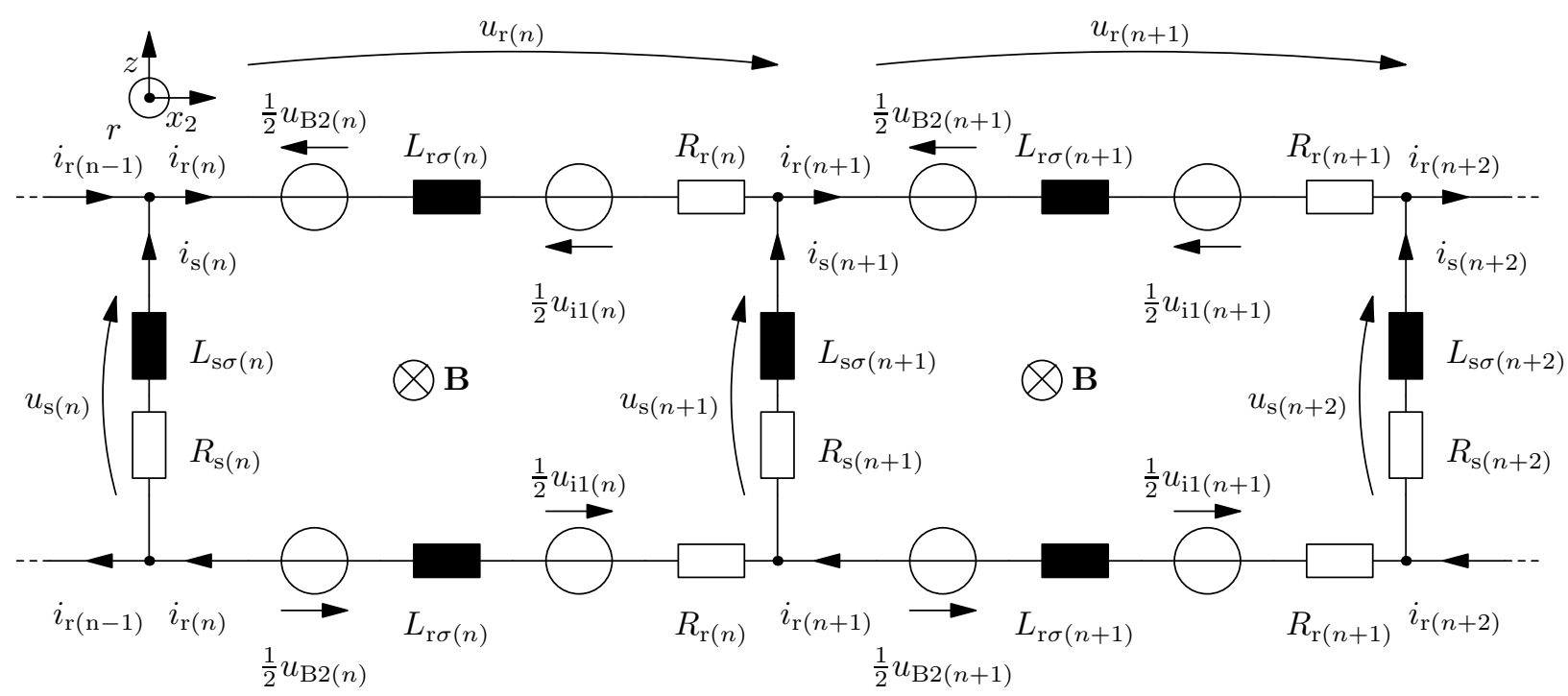

Bild 3.11: Ersatzschaltbild des Käfigs im Läufer

$$
\Rightarrow B_{2}\left(x_{2}, t\right)=\lambda\left(x_{2}, \rho\right) \cdot\left[i_{\mathrm{r}}\left(x_{2}, t\right)-\frac{\int_{0}^{2 \pi} \lambda\left(x_{2}, \rho\right) i_{\mathrm{r}}\left(x_{2}, t\right) \mathrm{d} x_{2}}{2 \pi \cdot \lambda_{0 \mu}(\rho)}\right]
$$

mit $\lambda_{0 \mu}(\rho)$ als dem von der Winkelkoordinate $x_{2}$ unabhängigen Teil des Luftspaltleitwerts sowie $\rho=\Omega t$.

Die Selbstinduktivität $L_{\delta}$ und die Gegeninduktionsspannung $u_{\mathrm{M}}$ aus dem Ersatzschaltbild der diskreten Betrachtung in Bild 3.4 lassen sich damit, wie in Bild 3.11] dargestellt, durch eine Spannung $u_{\mathrm{B} 2(n)}$, beziehungsweise bei Übergang zu unendlich vielen Stäben eine Spannungsdichte über $x_{2}$

$$
u_{\mathrm{B} 2}^{\prime}\left(x_{2}, t\right)=-r l_{\mathrm{i}} \cdot \frac{\mathrm{d} B_{2}\left(x_{2}, t\right)}{\mathrm{d} t}
$$

ersetzen. Dabei enthält $B_{2}\left(x_{2}, t\right)$ aufgrund der Abhängigkeit von $\lambda\left(x_{2}, \rho\right)$ bereits die mit (3.92) und (3.93) eingeführte Drehwinkelabhängigkeit der Induktivitäten. Eine Aufschlüsselung der Effekte nach Selbst- und Gegeninduktivitäten ist mit der hier aufgezeigten Darstellung nicht möglich, erscheint aber aufgrund des „Verschmelzens“ der realen Maschen auch nicht sinnvoll zu sein.

Entgegen der Auswertung von Maschengleichungen im diskreten Läufermodell, bietet sich im kontinuierlichen Modell die Berechnung über die Ringspannungsdichte $u_{\mathrm{r}}^{\prime}\left(x_{2}, t\right)$ an. Dabei gilt

$$
u_{\mathrm{r}}^{\prime}\left(x_{2}, t\right)=\mp r E_{\mathrm{r}}\left(x_{2}, t\right) \cdot \mathbf{e}_{\mathrm{x}_{2}},
$$

sodass es sich bei der Ringspannungsdichte um die auf den Radius normierte elektrische Feldstärke im Kurzschlussring handelt, welche in einem Kurzschlussring mit und im anderen Kurzschlussring entgegen der Winkelkoordinate $x_{2}$ gerichtet ist.

Die Spannungsdichte über den Ringwiderständen und -streuinduktivitäten, welche in Richtung der Koordinate $x_{2}$ betrachtet wird, ergibt sich aus dem Produkt des Ringstroms 
$i_{\mathrm{r}}\left(x_{2}, t\right)$ mit der Widerstands- beziehungsweise Streuinduktivitätsdichte des Kurzschlussrings, die sich bei gleichmäßig angeordneten Läufernuten zu

$$
R_{\mathrm{r}}^{\prime}\left(x_{2}\right)=\frac{N_{2} \bar{R}_{\mathrm{r}}}{2 \pi} \quad \text { und } \quad L_{\mathrm{r} \sigma}^{\prime}\left(x_{2}\right)=\frac{N_{2} \bar{L}_{\mathrm{r} \sigma}}{2 \pi}
$$

ergeben. Dabei sind $\bar{R}_{\mathrm{r}}$ und $\bar{L}_{\mathrm{r} \sigma}$ der durchschnittliche Widerstand beziehungsweise die durchschnittliche Streuinduktivität des Kurzschlussrings zwischen je zwei benachbarten Nuten.

Mit der Stabwiderstands- und Streuinduktivitätsverteilung, welche sich bei unmodulierten Läufernutpositionen und Breiten zu

$$
R_{\mathrm{s}}^{\prime}\left(x_{2}\right)=\frac{2 \pi \bar{R}_{\mathrm{s}(n)}}{N_{2}} \quad \text { bzw. } \quad L_{\mathrm{s} \sigma}^{\prime}\left(x_{2}\right)=\frac{2 \pi \bar{L}_{\mathrm{s} \sigma(n)}}{N_{2}}
$$

ergeben, und der Stabstromdichte $i_{\mathrm{s}}^{\prime}\left(x_{2}, t\right)$ gemäß (3.101) kann die Spannung über den unendlich vielen Läuferstäben berechnet werden. Es ergibt sich eine Stabspannungsverteilung $u_{\mathrm{s}}^{\prime}\left(x_{2}, t\right)$, deren Ableitung nach der Winkelkoordinate $x_{2}$ gerade der zweifachen Ringspannungsdichte $u_{\mathrm{r}}^{\prime}\left(x_{2}, t\right)$ entsprechen muss, damit sich Spannungsrichtigkeit in den unendlich vielen Käfigmaschen ergibt.

Zusammenfassend ergibt sich die Spannungsbeziehung

$$
\begin{aligned}
u_{\mathrm{r}}^{\prime}\left(x_{2}, t\right) & =R_{\mathrm{r}}^{\prime} \cdot i_{\mathrm{r}}\left(x_{2}, t\right)+L_{\mathrm{r} \sigma}^{\prime} \cdot \frac{\mathrm{d}}{\mathrm{d} t} i_{\mathrm{r}}\left(x_{2}, t\right)+\frac{r l_{\mathrm{i}}}{2} \cdot \frac{\mathrm{d}}{\mathrm{d} t}\left[B_{1}\left(x_{2}, t\right)+B_{2}\left(x_{2}, t\right)\right] \\
& =\frac{1}{2} \cdot \frac{\mathrm{d}}{\mathrm{d} x_{2}}[R_{\mathrm{s}}^{\prime} \cdot \underbrace{i_{\mathrm{s}}^{\prime}\left(x_{2}, t\right)}_{=\frac{\mathrm{d}}{\mathrm{d} x_{2}} i_{\mathrm{r}}\left(x_{2}, t\right)}+L_{\mathrm{s} \sigma}^{\prime} \cdot \frac{\mathrm{d}}{\mathrm{d} t} \underbrace{i_{\mathrm{s}}^{\prime}\left(x_{2}, t\right)}_{=\frac{\mathrm{d}}{\mathrm{d} x_{2}} i_{\mathrm{r}}\left(x_{2}, t\right)}]=\frac{1}{2} \cdot \frac{\mathrm{d}}{\mathrm{d} x_{2}} u_{\mathrm{s}}^{\prime}\left(x_{2}, t\right) .
\end{aligned}
$$

Hierbei ist zu beachten, dass die kontinuierliche Darstellung des Läufers lediglich eine Näherung eines realen Läufers mit $N_{2}$ Käfigstäben ist. Das heißt, dass der Ringstrom im realen Fall über jedem der $N_{2}$ Ringsegmente konstant und nicht wie bei der kontinuierlichen Betrachtung variabel ist. Damit wird ersichtlich, dass die kontinuierliche Betrachtung vornehmlich für niedrigere räumliche Ordnungszahlen mit Periodenweiten über mehrere Ringsegmente aussagekräftig ist.

\subsubsection{Berücksichtigung der realen Läuferzahnbreite}

In Abschnitt 3.6.5.1 wurde der Einfluss der realen Zahnbreite nicht berücksichtigt. Soll dies erfolgen, kann die partielle Differentialgleichung (13.111) in eine trigonometrische Summengleichung überführt werden, um die Anwendung des Kopplungsfaktors $\xi_{\mathrm{K}} \mathrm{zu}$ ermöglichen. Da dieser Faktor in seiner Definition (3.61) im Nenner die im Allgemeinen ortsabhängige Zahnbreite $b_{\mathrm{z}}\left(x_{2}\right)$ enthält, ist eine Darstellung, wie sie für den Einkopplungsfaktor $\xi_{\mathrm{u}}$ in (3.69) zu finden ist, für den Kopplungsfaktor nicht auf gleich einfachem Weg möglich. Wird jedoch die Spannungsgleichung der infinitesimal schmalen Käfigmaschen mit der Zahnbreite $b_{\mathrm{z}}\left(x_{2}\right)$ multipliziert, kann anstelle von $\xi_{\mathrm{K}}$ der Einkopplungsfaktor $\xi_{\mathrm{u}}$ gemäß (3.61) verwendet werden. 
Damit lässt sich der Ringstrom im Läufer, welcher sich aufgrund der Ständerinduktionswellen $B_{1}\left(x_{1}, t\right)=\sum_{\mu} \hat{B}_{\mu} \cdot \cos \left(\mu x_{1}-\omega_{1} t+\varphi_{\mu}\right)$ ergibt, mit dem Gleichungssystem berechnen, welches aus den Gleichungen

$$
\begin{array}{r}
b_{\mathrm{z}}\left(x_{2}\right) \cdot \sum_{n=-\infty}^{\infty}\left(2 R_{\mathrm{r}}^{\prime}\left(x_{2}\right)+n^{2} R_{\mathrm{s}}^{\prime}\left(x_{2}\right)+n \omega_{2 \mu} \frac{\mathrm{d} L_{\mathrm{s} \sigma}^{\prime}\left(x_{2}\right)}{\mathrm{d} x_{2}}\right) \cdot \hat{i}_{\mathrm{r} n} \cos \left(n x_{2}-\omega_{2 \mu} t+\varphi_{n}\right) \\
+b_{\mathrm{z}}\left(x_{2}\right) \cdot \sum_{n=-\infty}^{\infty}\left(n \frac{\mathrm{d} R_{\mathrm{s}}^{\prime}\left(x_{2}\right)}{\mathrm{d} x_{2}}+2 \omega_{2 \mu} L_{\mathrm{r} \sigma}^{\prime}\left(x_{2}\right)+n^{2} \omega_{2 \mu} L_{\mathrm{s} \sigma}^{\prime}\left(x_{2}\right)\right) \cdot \hat{i}_{\mathrm{r} n} \sin \left(n x_{2}-\omega_{2 \mu} t+\varphi_{n}\right) \\
+b_{\mathrm{z}}\left(x_{2}\right) \cdot \underbrace{r l_{\mathrm{i}} \cdot \frac{\mathrm{d} B_{2 \mu}\left(x_{2}, t\right)}{\mathrm{d} t}}_{=-u_{\mathrm{B} 2 \mu}^{\prime}\left(x_{2}, t\right)} \\
=-\hat{B}_{1 \mu} \cdot r l_{\mathrm{i}} \cdot \omega_{2 \mu} \cdot \underbrace{b_{\mathrm{z}}\left(x_{2}\right) \cdot \xi_{\mathrm{K} \mu}\left(x_{2}\right)}_{=\xi_{\mathrm{u} \mu}\left(x_{2}\right)} \cdot \xi_{\mathrm{schr} \mu}\left(x_{2}\right) \cdot \sin \left(\mu x_{2}-\omega_{2 \mu} t+\varphi_{\mu}\right)
\end{array}
$$

für verschiedene räumliche Ordnungszahlen und Frequenzen besteht. Hierbei wird der Ringstrom $i_{\mathrm{r}}\left(x_{2}, t\right)$ durch eine Fourierreihe verschiedener räumlicher Ordnungszahlen repräsentiert. Einschränkend sei für (3.112) erwähnt, dass diese Gleichungen jeweils ausschließlich für den Anteil der Läuferinduktion mit der Kreisfrequenz $\omega_{2 \mu}$, also $B_{2 \mu}\left(x_{2}, t\right)=$ $\hat{B}_{2 \mu} \cdot \cos \left(\omega_{2 \mu} t+\varphi_{\mathrm{B} \mu}\right)$, gelten.

Insbesondere gilt (3.112) für einen glatten Ständer, also einen über dem Winkel $x_{1}$ konstanten Luftspaltleitwert des Ständers $\lambda_{1}\left(x_{1}, t\right)$, womit sich

$$
u_{\mathrm{B} 2 \mu}^{\prime}\left(x_{2}, t\right)=-r l_{\mathrm{i}} \cdot \lambda_{0} \sum_{n=-\infty}^{\infty} \xi_{\mathrm{K} n}\left(x_{2}\right) \cdot \omega_{2 \mu} \hat{i}_{\mathrm{r} n} \sin \left(n x_{2}-\omega_{2 \mu} t+\varphi_{n}\right)
$$

ergibt.

Für konstante Werte von $\lambda, R_{\mathrm{s}}, L_{\mathrm{s} \sigma}, R_{\mathrm{r}}$ und $L_{\mathrm{r} \sigma}$ lässt sich die Lösung von (3.112) leicht analytisch bestimmen. Beim Auftreten von Harmonischen, insbesondere in $\lambda$, wird die analytische Lösung jedoch derart aufwändig, dass sich $\varphi_{n}$ und $i_{\mathrm{r} n}$ für endlich viele $n=$ $\left(1,2, \ldots, n_{\max }\right)$ besser numerisch bestimmen lassen. Deutlich einfacher gestaltet sich jedoch in den meisten Fällen, wie eingangs erwähnt, die Berechnung mit Hilfe des diskreten Modells aus Abschnitt 3.6.4.

Zur numerischen Lösung des Gleichungssystems lässt sich ausnutzen, dass die Beziehungen der Ströme und Spannungen im elektrischen Netzwerk des Läufers in (3.112) durch trigonometrische Summengleichungen dargestellt sind. Da sich die Sinusfunktionen mittels Verschiebung als Kosinusfunktionen darstellen lassen und diese wiederum für die verschiedenen Ordnungszahlen, also $\cos (n x+\varphi)$ mit $n=0, \pm 1, \pm 2, \ldots$, ein Orthogonalsystem bilden, lässt sich das mit (3.112) dargestellte Gleichungssystem eindeutig lösen.

Ähnlich wie bei einer diskreten Fourierreihenbildung wird die Systemgleichung (3.112) an den $2 k+1$ gleichverteilten Stützstellen $\tilde{x}_{0}$ bis $\tilde{x}_{2 k}$ mit $\tilde{x}_{n}=\frac{n \cdot 2 \pi}{2 k+1}$ diskretisiert, sodass sich ein Gleichungssystem zur Berechnung der unbekannten Ringströme der räumlichen Ord- 
nungszahlen $-k \leq n \leq k$ ergibt. Dieses Gleichungssystem wird mit der Impedanzmatrix

$$
\underline{\mathbf{Z}}=\left[\begin{array}{cccc}
Z_{-\mathrm{k}}^{\prime}\left(\tilde{x}_{0}\right) \cdot \mathrm{e}^{-\mathrm{j}(-k) \tilde{x}_{0}} & Z_{1-\mathrm{k}}^{\prime}\left(\tilde{x}_{0}\right) \cdot \mathrm{e}^{-\mathrm{j}(1-k) \tilde{x}_{0}} & \cdots & Z_{\mathrm{k}}^{\prime}\left(\tilde{x}_{0}\right) \cdot \mathrm{e}^{-\mathrm{j}(k) \tilde{x}_{0}} \\
Z_{-\mathrm{k}}^{\prime}\left(\tilde{x}_{1}\right) \cdot \mathrm{e}^{-\mathrm{j}(-k) \tilde{x}_{1}} & Z_{1-\mathrm{k}}^{\prime}\left(\tilde{x}_{1}\right) \cdot \mathrm{e}^{-\mathrm{j}(1-k) \tilde{x}_{1}} & \cdots & Z_{\mathrm{k}}^{\prime}\left(\tilde{x}_{1}\right) \cdot \mathrm{e}^{-\mathrm{j}(k) \tilde{x}_{1}} \\
\vdots & \vdots & \ddots & \vdots \\
Z_{-\mathrm{k}}^{\prime}\left(\tilde{x}_{2 k}\right) \cdot \mathrm{e}^{-\mathrm{j}(-k) \tilde{x}_{2 k}} & Z_{1-\mathrm{k}}^{\prime}\left(\tilde{x}_{2 k}\right) \cdot \mathrm{e}^{-\mathrm{j}(1-k) \tilde{x}_{2 k}} & \cdots & Z_{\mathrm{k}}^{\prime}\left(\tilde{x}_{2 k}\right) \cdot \mathrm{e}^{-\mathrm{j}(k) \tilde{x}_{2 k}}
\end{array}\right],
$$

welche

$$
R_{\mathrm{n}}^{\prime}\left(x_{2}\right)=2 R_{\mathrm{r}}^{\prime}\left(x_{2}\right)+n^{2} R_{\mathrm{s}}^{\prime}\left(x_{2}\right)+n \omega_{2 \mu} \frac{\mathrm{d} L_{\mathrm{s} \sigma}^{\prime}\left(x_{2}\right)}{\mathrm{d} x_{2}}
$$

und bei glattem Ständer

$$
X_{\mathrm{n}}^{\prime}\left(x_{2}\right)=n \frac{\mathrm{d} R_{\mathrm{s}}^{\prime}\left(x_{2}\right)}{\mathrm{d} x_{2}}+2 \omega_{2 \mu} L_{\mathrm{r} \sigma}^{\prime}\left(x_{2}\right)+n^{2} \omega_{2 \mu} L_{\mathrm{s} \sigma}^{\prime}\left(x_{2}\right)+r l_{\mathrm{i}} \omega_{2 \mu} \xi_{\mathrm{K} n} \cdot \lambda_{0}
$$

in der Form

$$
\underline{Z}_{\mathrm{n}}^{\prime}\left(x_{2}\right)=R_{\mathrm{n}}^{\prime}\left(x_{2}\right)+\mathrm{j} X_{\mathrm{n}}^{\prime}\left(x_{2}\right)
$$

enthält, zu

$$
\underline{\mathbf{Z}} \cdot\left[\begin{array}{c}
\underline{i}_{-k} \\
\underline{i}_{1-k} \\
\vdots \\
\underline{i}_{k}
\end{array}\right]=-\mathrm{j} r l_{\mathrm{i}} \omega_{2} \cdot \sum_{\mu} \hat{B}_{\mu} \cdot\left[\begin{array}{c}
\mathrm{si}\left(\frac{\mu \cdot b_{\mathrm{Z}}\left(\tilde{x}_{0}\right)}{2}\right) \cdot \mathrm{e}^{-\mathrm{j}\left(\mu \tilde{x}_{0}+\varphi_{1 \mu}\right)} \\
\mathrm{si}\left(\frac{\mu \cdot b_{\mathrm{Z}}\left(\tilde{x}_{1}\right)}{2}\right) \cdot \mathrm{e}^{-\mathrm{j}\left(\mu \tilde{x}_{1}+\varphi_{1 \mu}\right)} \\
\vdots \\
\operatorname{si}\left(\frac{\mu \cdot b_{\mathrm{Z}}\left(\tilde{x}_{2 k}\right)}{2}\right) \cdot \mathrm{e}^{-\mathrm{j}\left(\mu \tilde{x}_{2 k}+\varphi_{1 \mu}\right)}
\end{array}\right] \text {. }
$$

Bei glattem Ständer kann (3.118) jeweils für alle Induktionswellen der Ordnungszahlen $\mu$, welche zur selben Kreisfrequenz $\omega_{2}$ im Läufer führen, separat gelöst werden. Bei Vorhandensein signifikanter magnetischer Leitwertschwankungen aufgrund der Ständernutung muss hingegen die Frequenzkopplung aufgrund der Läuferrückwirkung berücksichtigt werden. Diese kann, genau wie bei der diskreten Berechnung beschrieben, iterativ berücksichtigt werden.

Der Ringstrom ergibt sich letztendlich zu

$$
i_{\mathrm{r}}\left(x_{2}, t\right)=\sum_{\omega_{2}} \sum_{n=-k}^{k}\left|\underline{\hat{i}}_{n}\right| \cdot \cos \left(n x_{1}-\omega_{2} t-\arg \left(\underline{i}_{n}\right)\right),
$$

sodass mit Hilfe von (3.102) die Felderregerwellen des kontinuierlich betrachteten Läufers berechnet werden können. 


\subsubsection{Wicklungsfaktor der Läuferwicklung}

Mit den in den letzten beiden Abschnitten gezeigten Verfahren lassen sich die Ringströme im Käfig des Läufers berechnen. Es soll nun geklärt werden, wie der diskret betrachtete, aber insbesondere auch der kontinuierlich betrachtete Käfig, der real aus diskreten Stäben besteht, aufgrund der in ihnen fließenden Ströme auf das Luftspaltfeld wirken.

Für die kontinuierliche Betrachtung wurde mit (3.103) und (3.106) bereits beschrieben, welche Luftspaltinduktion sich aufgrund der Ringströme ergibt. Die dort verwendeten Beziehungen haben den Vorteil, dass sie eine relativ einfache geschlossene analytische Weiterrechnung erlauben; sie vernachlässigen jedoch weitgehend den Einfluss der realen Nutpositionen. Hier soll nun mit diesem Vorteil der kontinuierlichen Darstellung gebrochen werden und der Strombelag, die Felderregung und letztendlich die Induktion durch eine Diskretisierung der kontinuierlich berechneten Ströme bestimmt werden.

Aus dem in (3.102) für das kontinuierliche Modell dargestellten Zusammenhang geht hervor, dass jede Welle des Ringstroms

$$
i_{\mathrm{r} \nu}\left(x_{2}, t\right)=\hat{i}_{\mathrm{r} \nu} \cos \left(\nu x_{2}-\omega_{\nu} t+\varphi_{\nu}\right)
$$

zu einer Felderregerwelle, also einer kontinuierlich veränderten Felderregung, sowie einer Welle der Stabstromdichte

$$
i_{\mathrm{s} \nu}^{\prime}\left(x_{2}, t\right)=\underbrace{\nu \hat{i}_{\mathrm{r} \nu}}_{=\hat{i}_{\mathrm{s} \nu}^{\prime}} \cos \left(\nu x_{2}-\omega_{\nu} t+\varphi_{\nu}+\frac{\pi}{2}\right)
$$

führt.

Beim realen Läufer mit der endlichen Stabzahl $N_{2}$ kann sich die Felderregung zwischen je zwei benachbarten Stäben nicht ändern. Bei Verwendung des kontinuierlichen Modells liegt es daher nahe, die Stabströme mittels Integration der Stabstromdichten zwischen je zwei benachbarten Zahnmitten zu berechnen. Es ergibt sich damit

$$
\begin{aligned}
i_{\mathrm{s} \nu(n)}(t) & =\int_{x_{2(n)}-\frac{b_{\mathrm{z}}}{2}}^{x_{2(n)}+\frac{b_{\mathrm{z}}}{2}} \hat{i}_{\mathrm{s} \nu}^{\prime} \cos \left(\nu x_{2}-\omega_{\nu} t+\varphi_{\nu}\right) \mathrm{d} x_{2} \\
& =\hat{i}_{\mathrm{s} \nu}^{\prime} \cdot \underbrace{\frac{\sin \left(\frac{\nu b_{\mathrm{z}}}{2}\right)}{\frac{\nu}{2}}}_{=\xi_{\mathrm{u} \nu}} \cos \left(\nu x_{2}-\omega_{\nu} t+\varphi_{\nu}\right),
\end{aligned}
$$

wobei, wie auch schon bei der induzierten Spannung, der Einkopplungsfaktor gemäß (3.61) in Erscheinung tritt.

Mit (3.123) liegen nun für die kontinuierliche wie auch für die diskrete Betrachtung des Läufers Werte für die einzelnen Stabströme vor. Hiermit können analog zum Vorgehen beim Ständer die läuferseitig erregten Anteile des Luftspaltfelds berechnet werden.

Die allgemein gehaltene Formel (3.8) ist direkt auf einen gleich- sowie auch ungleichmäßig genuteten Läufer übertragbar. Der Phasenverschiebungswinkel $\varphi_{(\mathrm{n})}$ in (3.8), welcher sich 
für einen diskretisierten Stab des kontinuierlichen Modells an der Position $x_{2(n)}$ aus (3.121) ergibt, ist bei einem Ringstrom der räumlichen Ordnungszahl $\nu$ gerade

$$
\varphi_{(\mathrm{n})}=-\nu x_{2(n)}-\varphi_{\nu}
$$

Damit wird (3.8) $\mathrm{zu}$

$$
\underline{A}_{2 \mu}\left(x_{2}, t\right)=\underbrace{\left[\sum_{n=1}^{N_{2}} \frac{1}{4 \pi} \cdot \xi_{\mathrm{N} \mu(n)} \hat{i}_{(n)} e^{\mathrm{j}\left(-\nu x_{2(n)}-\mu x_{2(n)}-\varphi_{\nu}\right)}\right]}_{=: \hat{A}_{2 \mu} \cdot e^{-\mathrm{j} \varphi \mu}} \cdot e^{\mathrm{j}\left(\mu x_{2}-\omega_{2} t\right)} .
$$

Für gleichmäßig verteilte Nuten mit elektrisch identischen Leitern wird die Summe in (3.125) $\mathrm{zu}$

$$
\hat{A}_{2 \mu} e^{-\mathrm{j} \varphi_{\mu}}=\frac{1}{4 \pi} \cdot \hat{i} \cdot \xi_{\mathrm{N} \mu} \cdot \sum_{n=1}^{N_{2}} e^{-\mathrm{j}(\nu+\mu) \frac{2 \pi n}{N_{2}}} \cdot e^{-\mathrm{j} \varphi_{\nu}}
$$

also nur für

$$
\nu+\mu=k N_{2} \quad \Leftrightarrow \quad \mu=k N_{2}-\nu \quad \text { mit } \quad k \in \mathbb{Z}
$$

ungleich Null, nämlich

$$
\hat{A}_{2 \mu}=\frac{\hat{i} \cdot \xi_{\mathrm{N} \mu} \cdot N_{2}}{4 \pi} \quad \text { und } \quad \varphi_{\mu}=\varphi_{\nu}
$$

Man erhält damit die Ordnungszahlen der sogenannten Läufernutharmonischen, welche bei Betrachtung im läuferfesten Koordinatensystem mit $x_{2}$ gelten. Werden die Strombelagswellen in ständerfesten Koordinaten dargestellt, so ergeben sich mit $x_{2}=x_{1}-\Omega t$ und der Kreisfrequenz $\omega_{\nu}$ des betrachteten Läuferstromanteils die Ordnungszahlen

$$
\mu=\left\{\begin{array}{ll}
k N_{2}-\nu & \text { für }\left(k N_{2}-\nu\right) \Omega \leq \omega_{\nu} \\
-\left(k N_{2}-\nu\right) & \text { für }\left(k N_{2}-\nu\right) \Omega>\omega_{\nu}
\end{array} .\right.
$$

Damit sind die Strombelagswellen bekannt, und die Felderregerwellen lassen sich analog $\mathrm{zu}(3.23)$ berechnen.

Da sich, wie in (3.100) zu sehen, die Kreisfrequenzen der Ringströme und somit auch die des Läuferstrombelags aus einer Linearkombination der positiven oder negativen Ständerkreisfrequenz $\omega_{1}$ sowie eines Vielfachen der Winkelgeschwindigkeit des Läufers $\Omega$ ergeben und sich bei Modulation der Felderregung mit den Leitwertwellen sowie aus der Transformation in das ständerfeste Koordinatensystem ausschließlich Variationen der Kreisfrequenz um Vielfache von $\Omega$ ergeben, besitzen auch alle läuferseitig erregten Induktionswellen eine Kreisfrequenz gemäß

$$
\omega_{\mathrm{B} 2 \nu}= \pm \omega_{1}+k \cdot \Omega \text { mit } k \in Z
$$




\subsubsection{Schrägung}

Der Einfluss der Schrägung auf die induzierte Spannung ist Abschnitt 3.6.1.3 zu entnehmen. Die weiteren im Ersatzschaltbild des Läuferkäfigs gemäß Bild 3.4 enthaltenen und von einer Schrägung abhängigen Größen sind $L_{\delta(n)}$ und $M_{n k}$ gemäß (3.92) und (3.93), wobei deren Schrägungsabhängigkeit bereits in ihren Definitionen durch den Schrägungsfaktor $\xi_{\text {schr }}$ berücksichtigt ist.

Mit den genannten schrägungsabhängigen Variablen erfolgt die Berechnung der Ströme im Läufer entsprechend einer ungeschrägten Geometrie. Es ist dabei zu beachten, dass der ortsabhängige Ringstrom $i_{\mathrm{r}}\left(x_{2}, t\right)$ jeweils für $z=0$ berechnet wird, bei Schrägung real jedoch um $\pm \frac{\gamma_{\text {schr }}}{2}$ verschoben auftritt.

Die Läuferfelderregung $v_{2}\left(x_{1}, t, i_{\mathrm{r}}\left(x_{2}, t\right)\right)$ ergibt sich damit zu

$$
v_{2}\left(x_{2}, t, z, i_{\mathrm{r}}\left(x_{2}, t\right)\right)=v_{2}\left(x_{2}-\frac{\gamma_{\mathrm{schr}}}{l_{\mathrm{i}}} z, t, i_{\mathrm{r}}\left(x_{2}, t\right)\right) .
$$

\subsubsection{Rückwirkung auf den Ständerstrom}

Die in den Abschnitten [3.1, 3.3 und 3.5 hergeleiteten Beziehungen der Ständerfelderregung sind von der Amplitude des Ständerstroms abhängig. Dieser hängt wiederum von der Ständerspannung, den Widerständen der Ständerwicklung sowie der in der Ständerwicklung induzierten Spannung ab. Die induzierte Spannung setzt sich dabei aus einer Selbstinduktionsspannung infolge von Nutstreuung und Ähnlichem, einer Selbstinduktionsspannung infolge der Haupt- und Oberwellen der Ständerwicklung sowie der von den Läuferinduktionswellen induzierten Spannung zusammen.

Für die praktische Umsetzung der Berechnung bedeutet dies, dass der Einfluss der Läuferrückwirkung auf die Ständerfelderregung bei kontinuierlicher Betrachtung geschlossen analytisch ermittelt werden kann. Alternativ lässt sich dieser iterativ berücksichtigen, wie es bei den Berechnungen in Kapitel 7 grundsätzlich durchgeführt wurde. Bei dieser für die diskrete Betrachtung vorteilhaften Methode wird nach der Berechnung der Läuferrückwirkung und der daraus resultierenden induzierten Spannung in der Ständerwicklung der Ständerstrom für die nächste Iterationsschleife angepasst. 


\section{Geräuschanregung und Drehmomentbildung}

Die Hauptursachen für magnetische Geräuschanregungen sind insbesondere Radialkraftwellen aber auch Pendeldrehmomente sowie lokale tangentiale Kraftanregungen an den Zähnen.

Der Fokus in diesem Kapitel soll auf der Berechnung der geräuschanregenden Kräfte, also den gerade genannten Radialkraftwellen und den tangentialen Kräften an den Ständerzähnen liegen, welche letztendlich zu Jochzugwellen führen. Die wesentlichen Zusammenhänge zur Berechnung der daraus resultierenden Schallabstrahlung werden anschließend in Abschnitt 4.5 kurz aufgezeigt. Des Weiteren wird die Berechnung von Pendeldrehmomenten vorgestellt, die damit verbundene Schallabstrahlung jedoch nicht weiter beleuchtet.

Vereinfachend werden bei der Berechnung der anregenden Kräfte leere Nuten, über die keine Kräfte zu den Nachbarzähnen übertragen werden können, angenommen. Eine genauere, aber auch wesentlich aufwändigere Modellierung des Ständers müsste die Wicklung als Dämpfer-Feder-System berücksichtigen, sodass eine tangential an einem Zahn angreifende Kraft über dessen Verbiegung auch zu Tangentialkräften an den Nachbarzähnen führt. Der Fehler, der durch diese auch von Weh und Wachta [32,33] durchgeführte Vereinfachung entsteht, zeigt sich insbesondere bei tangentialen Kraftanregungen höherer räumlicher Ordnungszahlen. Bei niedrigeren Ordnungszahlen wirken die Tangentialkräfte benachbarter Zähne hingegen annähernd simultan, sodass eine Verbindung beider Zähne nahezu wirkungslos bliebe. Die hier genannte Vereinfachung bezieht sich dabei ausschließlich auf die Berechnung der anregenden Kräfte. Bei der Berechnung der von den Jochzugwellen herrührenden Schwingungen wird die Wicklung hingegen in Form ihrer Masse und einer Dämpfung mit einbezogen.

Die Berechnung von Geräuschanregungen und die Bewertung ihrer Wirkung auf den Menschen erlaubt eine Beschränkung der Frequenzen auf den hörbaren Bereich, also etwa $16 \mathrm{~Hz}$ bis $20 \mathrm{kHz}$. Handelt es sich um eine drehzahl- und speisefrequenzvariable Maschine, so führt jedoch praktisch jede Anregung in bestimmten Betriebspunkten zu Geräuschemissionen in diesem Frequenzbereich, sodass sich keine Beschränkung des Berechnungsumfangs ergibt. Ausgenommen sind hiervon Maschinen, die unterhalb der hörbaren Frequenz arbeiten.

\subsection{Drehmoment}

Zur Berechnung des Drehmoments der Maschine wird die Energie des magnetischen Felds herangezogen. Diese bestimmt sich entsprechend der zum Beispiel in [15] angegebenen 
Beziehung über das Volumenintegral

$$
W_{\mathrm{mag}}=\frac{1}{2} \iiint \mathbf{B} \cdot \mathbf{H} \mathrm{d} V
$$

Die magnetisch wirksame Luftspaltweite $\delta^{*}\left(x_{1}, t\right)$, welche die Nutung von Ständer und Läufer berücksichtigt, ist im Allgemeinen über $x_{1}$ nicht konstant, weshalb sich bei konstanter Permeabilität des Luftspalts $\mu_{\delta}=\mu_{0}$ die in Abschnitt 3.4 eingeführte nichtkonstante magnetische Leitwertverteilung $\lambda\left(x_{1}, t\right)$ ergibt. Um eine einfachere Berechnung der magnetischen Feldenergie und damit auch des Drehmoments zu ermöglichen, kann die in der Realität ortsabhängige Luftspaltweite $\delta^{*}\left(x_{1}, t\right)$ bei konstanter magnetischen Permeabilität der Luft $\mu_{\delta}$, ähnlich wie in [24], auf die konstante Luftspaltweite $\delta=r_{\mathrm{i} 1}-r_{\mathrm{a} 2}$ bei der fiktiven zeit- und ortsabhängigen Permeabilität $\mu_{\delta}\left(x_{1}, t\right)$ umgerechnet werden. Es gilt dann

$$
\mu_{\delta}\left(x_{1}, t\right)=\delta \cdot \lambda\left(x_{1}, t\right)
$$

Vereinfachend wird die magnetische Induktion $\mathbf{B}$ und entsprechend auch die magnetische Feldstärke $\mathbf{H}$ im Luftspalt als rein radial angesehen. Diese Vereinfachung ist bei engen Luftspalten und geringer Sättigung der Ständer- und Läuferzähne aufgrund des Verhältnisses der magnetischen Permeabilitäten von Eisen und Luft $\left(\mu_{\mathrm{r}, \mathrm{Fe}} \mu_{0} \gg \mu_{0}\right)$ legitim. Die Feldlinien treten dabei annähernd senkrecht aus dem Eisen aus und somit nahezu rein radial über den Luftspalt:

$$
\mathbf{B}=B_{\mathrm{r}} \mathbf{e}_{\mathrm{r}} \quad \mathbf{H}=H_{\mathrm{r}} \mathbf{e}_{\mathrm{r}} .
$$

Mit den Abhängigkeiten der Induktion von der magnetischen Permeabilität und Feldstärke

$$
B\left(x_{1}, t\right)=\mu_{\delta}\left(x_{1}, t\right) \cdot H\left(x_{1}, t\right)
$$

beziehungsweise vom magnetischen Leitwert und der Felderregung

$$
B\left(x_{1}, t\right)=\lambda\left(x_{1}, t\right) \cdot V\left(x_{1}, t\right)
$$

lässt sich (4.1) somit in

$$
W_{\text {mag }}=\frac{D}{4 \delta} \int_{-\frac{l_{1}}{2}}^{\frac{l_{\mathrm{i}}}{2}} \int_{r_{\mathrm{a} 2}}^{r_{\mathrm{i} 1}} \int_{0}^{2 \pi} \lambda\left(x_{1}, t\right) \cdot V^{2}\left(x_{1}, t\right) \mathrm{d} x_{1} \mathrm{~d} r \mathrm{~d} z=\frac{D l_{\mathrm{i}}}{4} \int_{0}^{2 \pi} \lambda\left(x_{1}, t\right) \cdot V^{2}\left(x_{1}, t\right) \mathrm{d} x_{1}
$$

überführen. Das Drehmoment der Maschine lässt sich daraus durch eine virtuelle Verschiebung, also über eine Differenziation nach der zusätzlich eingeführten zeitunabhängigen 
Variable $\rho_{0}$, dem Drehwinkel des Läufers zum Zeitpunkt $t=0$, bestimmen. Es ergibt sich

$$
\begin{aligned}
M(t) & =\frac{D l_{\mathrm{i}}}{4} \int_{0}^{2 \pi} \frac{\mathrm{d}}{\mathrm{d} \rho_{0}}\left[\lambda\left(x_{1}, t, \rho_{0}\right) \cdot V^{2}\left(x_{1}, t, \rho_{0}\right)\right] \mathrm{d} x_{1} \\
& =\frac{D l_{\mathrm{i}}}{4} \int_{0}^{2 \pi} \frac{\mathrm{d} \lambda\left(x_{1}, t, \rho_{0}\right)}{\mathrm{d} \rho_{0}} \cdot V^{2}\left(x_{1}, t, \rho_{0}\right) \mathrm{d} x_{1} \\
& +\frac{D l_{\mathrm{i}}}{2} \int_{0}^{2 \pi} \underbrace{\frac{\mathrm{d} V\left(x_{1}, t, \rho_{0}\right)}{\mathrm{d} \rho_{0}}}_{=\frac{D}{2} A_{2}^{*}\left(x_{1}, t\right)} \cdot \underbrace{V\left(x_{1}, t, \rho_{0}\right) \cdot \lambda\left(x_{1}, t, \rho_{0}\right)}_{=B\left(x_{1}, t\right)} \mathrm{d} x_{1},
\end{aligned}
$$

wobei

$$
V\left(x_{1}, t, \rho_{0}\right)=V_{1}\left(x_{1}, t\right)+V_{2}\left(x_{1}-\rho_{0}, t\right)
$$

und

$$
\lambda\left(x_{1}, t, \rho_{0}\right)=\lambda\left(x_{1}, t+\frac{\rho_{0}}{\Omega}\right)
$$

gelten. Dabei stellt die in (4.9) enthaltene Division durch $\Omega$ auch bei $\Omega=0$ kein numerisches Problem dar, weil durch die zweidimensionale schnelle Fouriertransformation bei Bestimmung der Luftspaltleitwertwellen eigentlich $\lambda\left(x_{1}, \rho\right)$ mit $\rho=\rho_{0}+\Omega t$ und nicht $\lambda\left(x_{1}, t\right)$ berechnet wird. Die Transformation gemäß (4.9) kann folglich bei direkter Verwendung von $\lambda\left(x_{1}, \rho\right)$ umgangen werden.

Zusammenfassend lässt sich das auf diese Weise hergeleitete Drehmoment als Summe der beiden Anteile

$$
M_{1}(t)=\frac{D^{2}}{4} l_{\mathrm{i}} \int_{0}^{2 \pi} A_{2}^{*}\left(x_{1}, t\right) B\left(x_{1}, t\right) \mathrm{d} x_{1}
$$

und

$$
\begin{array}{r}
M_{2}(t)=\frac{D l_{\mathrm{i}}}{4} \int_{0}^{2 \pi} \frac{\mathrm{d} \lambda\left(x_{1}, \rho\right)}{\mathrm{d} \rho} \cdot V^{2}\left(x_{1}, t\right) \mathrm{d} x_{1} \\
\stackrel{\Omega \neq 0}{=} \frac{D l_{\mathrm{i}}}{4 \Omega} \int_{0}^{2 \pi} \frac{\mathrm{d} \lambda\left(x_{1}, t\right)}{\mathrm{d} t} \cdot V^{2}\left(x_{1}, t\right) \mathrm{d} x_{1}
\end{array}
$$

darstellen.

Ist der Läufer der Maschine ideal glatt, ist also $\lambda_{2}\left(x_{1}, t\right)$ über $x_{1}$ konstant, so wird (4.11) zu Null, und (4.10) bildet das Drehmoment korrekt ab. Zur Einbeziehung des Reluktanzdrehmoments von Maschinen mit ausgeprägten Polen im Läufer wäre hingegen der umgekehrte Fall von Interesse, sodass das Drehmoment einer ständerseitig glatten Maschine mit nur einer einfachen Formel abgebildet werden kann. 
Dies lässt sich erreichen, wenn zur Herleitung des Drehmoments nicht eine Läuferdrehung aus einer ständerfesten Position, sondern die Drehung des Läufers aus einer läuferfesten Position betrachtet wird. Es lassen sich so analog zu den vorhergehenden Ausführungen die Zusammenhänge

und

$$
\begin{aligned}
& M_{1}^{*}(t)=-\frac{D^{2}}{4} l_{\mathrm{i}} \int_{0}^{2 \pi} A_{1}^{*}\left(x_{1}, t\right) B\left(x_{1}, t\right) \mathrm{d} x \\
& M_{2}^{*}(t)=\frac{D l_{\mathrm{i}}}{4} \int_{0}^{2 \pi} \frac{\mathrm{d} \lambda\left(x_{2}+\rho_{0}+\Omega t, \rho\right)}{\mathrm{d} \rho_{0}} \cdot V^{2}\left(x_{1}, t\right) \mathrm{d} x_{1}
\end{aligned}
$$

finden. $M_{1}^{*}(t)$ bildet dabei das Drehmoment von Maschinen mit ausgeprägten Polen am Läufer und im Verhältnis dazu glattem Ständer weitgehend genau ab.

Wird die Luftspaltleitwertverteilung in (4.11) mit der Vereinfachung aus (3.42) dargestellt, so ergibt sich die Ableitung nach dem Läuferverdrehwinkel $\rho$ zu

$$
\begin{aligned}
\frac{\mathrm{d} \lambda^{\prime \prime}\left(x_{1}, \rho\right)}{\mathrm{d} \rho} & =\underbrace{\frac{\frac{-2 \mu_{0}}{\delta \lambda_{10}-2 \mu_{0}} \cdot \frac{1}{\delta \lambda_{20}-2 \mu_{0}}}{\frac{\lambda_{10}}{\delta \lambda_{10}-2 \mu_{0}}+\frac{\lambda_{20}}{\delta \lambda_{20}-2 \mu_{0}}} \sum_{\nu=0}^{\infty} \sum_{\mu=0}^{\infty} \mu \hat{\lambda}_{1 \nu} \hat{\lambda}_{2 \mu} \underbrace{\cos \left(\nu x_{1}+\varphi_{\lambda \nu}\right)}_{\text {Ständer }} \cdot \underbrace{\sin \left(\mu x_{1}-\mu \rho+\varphi_{\lambda \mu}\right)}_{\text {Läufer }}}_{=: \frac{\lambda_{0}^{\prime \prime}}{\lambda_{10} \cdot \lambda_{20}}} \\
& =-\frac{\lambda_{0}^{\prime \prime}}{\lambda_{10} \cdot \lambda_{20}} \cdot \lambda_{1}\left(x_{1}\right) \cdot \frac{\mathrm{d} \lambda_{2}\left(x_{1}, \rho\right)}{\mathrm{d} x_{1}}=-\frac{\lambda_{0}^{\prime \prime}}{\lambda_{10} \cdot \lambda_{20}} \cdot \lambda_{1}\left(x_{1}\right) \cdot \frac{\mathrm{d} \lambda_{2}\left(x_{2}\right)}{\mathrm{d} x_{2}} .
\end{aligned}
$$

Damit lässt sich $M_{2}(t)$ näherungsweise als

$$
M_{2}(t)=-\frac{\lambda_{0}^{\prime \prime}}{\lambda_{10} \cdot \lambda_{20}} \cdot \frac{D l_{\mathrm{i}}}{4} \int_{0}^{2 \pi} \frac{\mathrm{d} \lambda_{2}\left(x_{2}\right)}{\mathrm{d} x_{2}} \cdot \lambda_{1}\left(x_{1}\right) \cdot V^{2}\left(x_{1}, t\right) \mathrm{d} x_{1}
$$

darstellen, was zwar anschaulich ist, numerisch jedoch keine Vorteile bietet, da zur Berechnung der Felderregung des Läufers ohnehin die Reihenschaltung der Ständer- und Läuferleitwertverteilungen $\lambda_{1}\left(x_{1}, t\right)$ und $\lambda_{2}\left(x_{1}, t\right)$ berechnet werden muss. Sie liegt daher zur Drehmomentberechnung ohne erneuten Rechenaufwand bereits vor.

Kann der Einfluss des Ständers auf den magnetischen Luftspaltleitwert vernachlässigt, der Ständer also als glatt angesehen werden, gilt

$$
\lambda\left(x_{1}, \rho\right)=\lambda_{2}\left(x_{1}, \rho\right)
$$

sodass sich (4.15) $\mathrm{zu}$

$$
M_{2}(t)=-\frac{D l_{\mathrm{i}}}{4} \int_{0}^{2 \pi} \frac{\mathrm{d} \lambda_{2}\left(x_{2}\right)}{\mathrm{d} x_{2}} \cdot V^{2}\left(x_{1}, t\right) \mathrm{d} x_{1}
$$

vereinfacht. Mit dieser vereinfachenden Annahme entspricht der in diesem Abschnitt hergeleitete Zusammenhang für das Drehmoment dem in [24] hergeleiteten. 


\subsubsection{Schrägung}

Wird mit nicht schrägungsabhängigen Felderregungen und Leitwertverteilungen gerechnet, so kann das Drehmoment bei geschrägter Maschine näherungsweise nach Zerlegung der Felderregung in die ständer- und die läuferseitigen Anteile berechnet werden. Es sind die Beziehungen

$$
\lambda\left(x_{1}, \rho, z\right)=\lambda\left(x_{1}, \rho+\frac{\gamma_{\mathrm{schr}}}{l_{\mathrm{i}}} z\right)
$$

und

$$
V\left(x_{1}, t, z\right)=V_{1}\left(x_{1}, t\right)+V_{2}\left(x_{1}, t, z\right)=V_{1}\left(x_{1}, t\right)+V_{2}\left(x_{1}-\frac{\gamma_{\mathrm{schr}}}{l_{\mathrm{i}}} z, t\right)
$$

anzuwenden.

Eine genauere Alternative zur Berechnung des Drehmoments geschrägter Maschinen ist die Verwendung von bereits schrägungsbehafteten Eingangsgrößen, in denen die Schrägung mittels des Schrägungskoeffizienten $\sigma$ berücksichtigt ist. Die Integration in (4.6) über die Länge des Aktivteils der Maschine führt damit zum Beispiel in (4.10) zu den zusätzlichen Faktoren

$$
\xi_{\mathrm{sr} \sigma A B}=\frac{\sin \left(\frac{\sigma_{\mathrm{A}}-\sigma_{\mathrm{B}}}{2}\right)}{\frac{\sigma_{\mathrm{A}}-\sigma_{\mathrm{B}}}{2}} .
$$

Dabei ist $\sigma_{\mathrm{A}}$ der Schrägungskoeffizient einer Strombelags- und $\sigma_{\mathrm{B}}$ der Schrägungskoeffizient einer Induktionswelle.

Neben einer Reduktion des resultierenden Drehmoments kommt es bei Schrägung aufgrund der über der Maschinenlänge verschiedenen beziehungsweise phasenverschobenen Drehmomente zu Torsionskräften, welche hier jedoch nicht weiter betrachtet werden sollen.

\subsubsection{Drehmoment des kontinuierlichen Modells}

Mit der kontinuierlichen Beschreibung des Läufers lässt sich die Hauptwelle des orts- und zeitabhängigen Ringstroms $i_{\mathrm{r}}\left(x_{2}, t\right)$ für annähernd glatte Ständer- und Läuferoberflächen direkt aus der Ständerinduktionshauptwelle zu

$$
\underline{\hat{i}}_{\mathrm{r} p}\left(\underline{B}_{1 p}\right)=\frac{\mathrm{j} \omega_{2} \cdot r l_{\mathrm{i}} \cdot \xi_{\mathrm{K} p} \cdot \xi_{\mathrm{schr} p} \cdot \underline{B}_{1 p}}{\left(2 R_{\mathrm{r}}^{\prime}+p^{2} R_{\mathrm{s}}^{\prime}\right)-\mathrm{j} \omega_{2}\left(2 L_{\mathrm{r} \sigma}^{\prime}+p^{2} L_{\mathrm{s} \sigma}^{\prime}+r l_{\mathrm{i}} \lambda_{0} \xi_{\mathrm{K} p}\right)}
$$

berechnen. Daraus ergibt sich mit (4.10) beispielhaft das von $\hat{B}_{1 p}$ und dem Schlupf $s$ abhängige stationäre Drehmoment zu

$$
M_{1}\left(\hat{B}_{1 p}, s\right)=\frac{2 \pi \cdot s \omega_{1} \cdot r^{2} l_{\mathrm{i}}^{2} \cdot \xi_{\mathrm{K} p}^{2} \cdot \xi_{\mathrm{schr} p}^{2} \cdot \hat{B}_{1 p}^{2} \cdot\left(2 R_{\mathrm{r}}^{\prime}+p^{2} R_{\mathrm{s}}^{\prime}\right)}{\left(2 R_{\mathrm{r}}^{\prime}+p^{2} R_{\mathrm{s}}^{\prime}\right)^{2}+s^{2} \omega_{1}^{2} \cdot\left(2 L_{\mathrm{r} \sigma}^{\prime}+p^{2} L_{\mathrm{s} \sigma}^{\prime}+r l_{\mathrm{i}} \lambda_{0} \xi_{\mathrm{K} p}\right)^{2}} .
$$




\subsection{Radialkraftwellen}

Wie eingangs in diesem Kapitel erwähnt, führen insbesondere radial am Ständer angreifende Kräfte zu signifikanten Geräuschen. Das später verwendete Modell nutzt dabei die Kräfte auf das Ständerjoch als Eingangsgrößen. Zu deren Berechnung müssen zunächst die radialen Zugspannungen im Luftspalt bestimmt werden, welche sich gemäß [15] zu

$$
\sigma_{\mathrm{r}}\left(x_{1}, t\right)=\frac{1}{2 \mu_{0}} B^{2}\left(x_{1}, t\right)
$$

beziehungsweise mit der Darstellung als Feldwellen zu

$$
\begin{aligned}
\sigma_{\mathrm{r}}\left(x_{1}, t\right) & =\frac{1}{2 \mu_{0}} \sum_{\mu, \omega_{\mu}} \sum_{\nu, \omega_{\nu}} \hat{B}_{\mu} \cos \left(\mu x_{1}-\omega_{\mu} t+\varphi_{\mu}\right) \hat{B}_{\nu} \cos \left(\nu x_{1}-\omega_{\nu} t+\varphi_{\nu}\right) \\
& =\frac{1}{2 \mu_{0}} \sum_{\mu, \omega_{\mu}} \sum_{\nu, \omega_{\nu}} \frac{\hat{B}_{\mu} \hat{B}_{\nu}}{2}\left[\begin{array}{c}
\cos \left((\mu+\nu) x_{1}-\left(\omega_{\mu}+\omega_{\nu}\right) t+\left(\varphi_{\mu}+\varphi_{\nu}\right)\right)+\ldots \\
\ldots+\cos \left((\mu-\nu) x_{1}-\left(\omega_{\mu}-\omega_{\nu}\right) t+\left(\varphi_{\mu}-\varphi_{\nu}\right)\right)
\end{array}\right]
\end{aligned}
$$

ergeben. Die betragsmäßig größten Amplituden entstehen dabei in der Regel aus der Induktionshauptwelle sowie aus den nutharmonischen Induktionswellen.

Für Maschinen mit Luftspaltwicklung, also ungenutetem Ständer, kann aus (4.24) direkt die Kraftwirkung auf das Ständerjoch und damit die Schwingungsanregung berechnet werden. Im allgemeinen Fall ständerseitig genuteter Maschinen findet jedoch eine Modulation durch die Ständerzähne statt. Das bedeutet, dass die magnetischen Kräfte zunächst auf die einzelnen Zähne wirken und von diesen auf das Joch übertragen werden.

Die Wirkung der Kräfte auf einen Zahn ist in Bild4.1a skizziert. Vereinfachend wird dort die Flächenkraft am Zahnkopf durch die drei Kräfte $\mathbf{F}_{\mathrm{r} 1}$ bis $\mathbf{F}_{\mathrm{r} 3}$ repräsentiert. Die Kraft $\mathbf{F}_{\mathrm{r} 2}$ stellt dabei eine Kraft dar, welche aufgrund ihrer Lage in der Mitte eines Zahns quasi direkt auf das Joch wirkt. Die im Randbereich des Zahns angreifenden Kräfte $\mathbf{F}_{\mathrm{r} 1}$ und $\mathbf{F}_{\mathrm{r} 3}$ führen hingegen zu einer am Umfang verschobenen Jochkraft sowie zu einem Drehmoment. In Bild 4.1a nicht zu erkennen sind die nach (4.24) berechneten Zugkräfte im Bereich der Nuten. Diese können nicht an der Nut angreifen, sondern müssen über die benachbarten Zähne auf das Ständerblechpaket wirken, womit sie wie $\mathbf{F}_{\mathrm{r} 1}$ und $\mathbf{F}_{\mathrm{r} 3}$ betrachtbar sind.

Da in der Realität nicht die drei in Bild 4.1a dargestellten diskreten Kräfte, sondern eine Flächenkraft wirkt, ergibt sich die rein radial betrachtete Zahnkraft $F_{z}$ aus einer Integration über eben diese für die Mitte des Luftspalts berechnete Flächenkraft im Bereich der einzelnen Zähne sowie jeweils, nach willkürlicher Annahme, der halben angrenzenden Nuten zu

$$
F_{\mathrm{z}(n)}=l_{\mathrm{i}} r \cdot \sum_{\mu} \sum_{\nu} \int_{x_{1(n)}}^{x_{1(n+1)}} \hat{\sigma}_{\mu \nu} \cdot \cos \left(\mu x_{1}-\omega_{\nu} t+\varphi_{\mu \nu}\right) \mathrm{d} x_{1} .
$$

Für den Fall gleichmäßsig angeordneter Ständernuten mit $x_{1(n)}=\frac{2 \pi}{N_{1}} \cdot n$ ergibt sich daraus

$$
F_{\mathrm{z}(n)}=2 l_{\mathrm{i}} r \cdot \sum_{\mu} \sum_{\nu} \frac{\hat{\sigma}_{\mu \nu}}{\mu} \cdot \cos \left(\mu \frac{(2 n+1) \cdot \pi}{N_{1}}-\omega_{\nu} t+\varphi_{\mu \nu}\right) \cdot \sin \left(\mu \frac{\pi}{N_{1}}\right)
$$




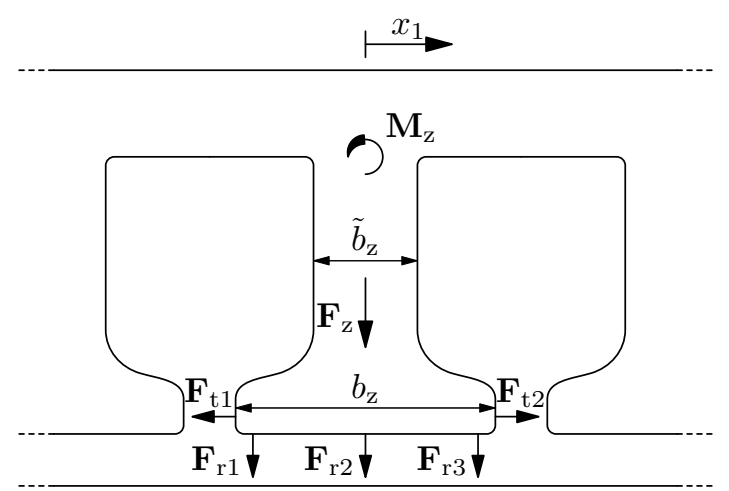

(a) Mit Zahndrehmoment

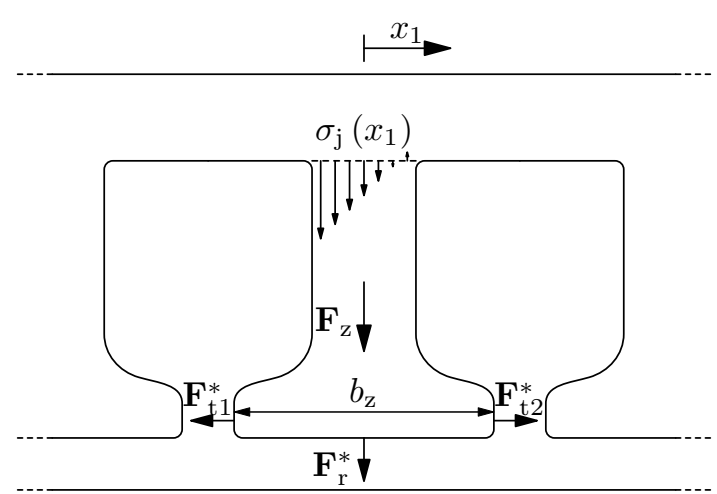

(b) Umgerechnete Kräfte und Drehmomente

Bild 4.1: Krafteinleitung in das Joch

womit die Zahnkräfte für die Zugspannungswellen $\mu=k \cdot N_{1}$ mit $k \in \mathbb{Z} \backslash\{0\}$ gerade zu Null werden.

Neben der Kraft $F_{z(n)}$ ergibt sich an jedem Ständerzahn ein ausschließlich auf den Radialkraftwellen basierendes Drehmoment, welches sich zu

$$
\begin{aligned}
M_{\mathrm{rz}(n)}= & l_{\mathrm{i}} r^{2} \cdot \sum_{\mu} \sum_{\nu} \frac{\hat{\sigma}_{\mu \nu}}{\mu^{2}} \cdot \sin \left(\mu \frac{x_{1(n)}+x_{1(n+1)}}{2}-\omega_{\nu} t+\varphi_{\mu \nu}\right) \\
& \cdot\left[\mu\left(x_{1(n)}-x_{1(n+1)}\right) \cdot \cos \left(\mu \frac{x_{1(n+1)}-x_{1(n)}}{2}\right)+2 \sin \left(\mu \frac{x_{1(n+1)}-x_{1(n)}}{2}\right)\right]
\end{aligned}
$$

bestimmen lässt. Dieses wird bei gleichmäßiger Ständernutung zu

$$
\begin{aligned}
& M_{\mathrm{rz}(n)}=-2 l_{\mathrm{i}} r^{2} \cdot \sum_{\mu} \sum_{\nu} \frac{\hat{\sigma}_{\mu \nu}}{\mu^{2}} \cdot\left[\sin \left(\mu \frac{\pi}{N_{1}}\right)-\mu \frac{\pi}{N_{1}} \cdot \cos \left(\mu \frac{\pi}{N_{1}}\right)\right] \\
& \cdot \sin \left(\mu \frac{(2 n+1) \cdot \pi}{N_{1}}-\omega_{\nu} t+\varphi_{\mu \nu}\right) \text {. }
\end{aligned}
$$

Die Amplitude der Drehmomente $M_{\mathrm{rz}(n)}$ wird dabei vom Kosinusterm in (4.28) dominiert, sodass sich Maxima nahe $\mu=k \cdot N_{1}$ mit $k \in \mathbb{Z} \backslash\{0\}$ ergeben. Folglich liegen maximale Drehmomente gerade für die Ordnungszahlen $\mu$ vor, für die sich minimale Zahnkräfte ergeben.

\subsection{Tangentialkräfte}

Jede relativ zu Ständer oder Läufer umlaufende Induktionswelle führt neben den im letzten Abschnitt aufgezeigten Radialkräften zu zeitvarianten Tangentialkräften an den Zähnen. Hieraus ergeben sich Zahndrehmomente und ebenso Schwingungsanregungen des Jochs.

Neben den Zahndrehmomenten führen die Tangentialkräfte an den Zähnen selbstverständlich auch zu in $x_{1}$-Richtung wirkenden Jochkräften, nämlich $F_{\mathrm{t} 2}-F_{\mathrm{t} 1}$, welche zu Stauchun- 


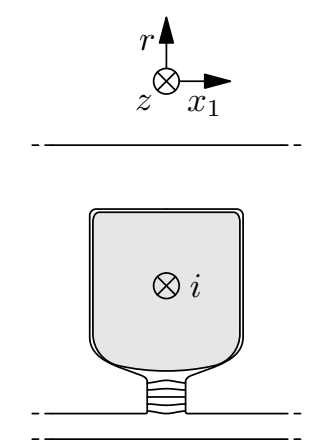

(a) Bestromte Nut; keine Luftspaltinduktion vorhanden

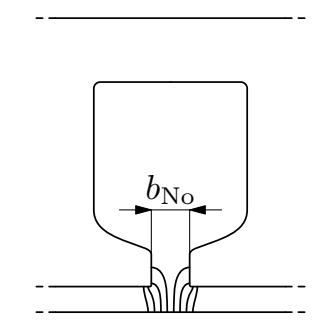

(b) Unbestromte Nut; Luftspaltinduktion vorhanden

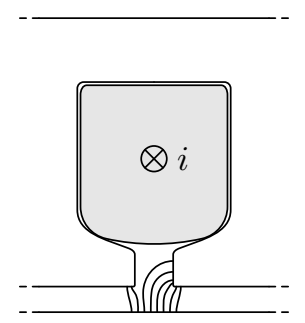

(c) Bestromte Nut; Luftspaltinduktion vorhanden

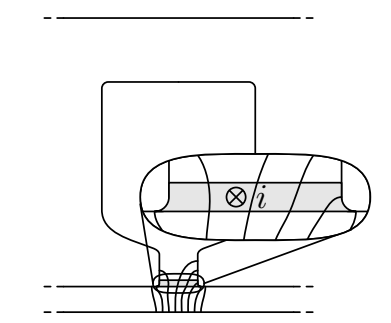

(d) Strombelag in der Nut; Luftspaltinduktion vorhanden; Ausschnittvergrößerung des Ersatzleiters

Bild 4.2: Schematische Darstellung der Induktion im Bereich der Nuten

gen beziehungsweise Streckungen des Jochs sowie dem Drehmoment der Maschine führen. Aufgrund eines geringen zu erwartenden Einflusses, sollen dabei die nichtdrehmomentbildenden Anteile der Tangentialkräfte am Joch nicht in die Geräuschberechnung einbezogen werden.

Um nun die Tangentialkräfte an den Zähnen sowie die daraus resultierenden Zahndrehmomente zu berechnen, sollen zunächst die in Bild 4.2c und Bild 4.2d schematisch dargestellten Feldlinien innerhalb einer Nut betrachtet werden. Dargestellt sind dabei zum einen die realen Feldlinien, welche sich in einer stromdurchflossenen Nut bei Felderregung des gegenüberliegenden Hauptelements ergeben, sowie die Feldlinien der Modellanordnung mit einem Strombelag gemäß den Ausführungen in Abschnitt 3.1, welcher hier durch einen flachen luftspaltnahen Ersatzleiter angenähert ist. Es wird im Vergleich direkt ersichtlich, dass sich an den jeweiligen Zahnflanken in beiden Anordnungen verschiedene Induktionen und somit auch magnetische Kräfte ergeben, welche zudem auf unterschiedlicher Zahnhöhe angreifen und somit über unterschiedliche Hebelarme in die Zahndrehmomente eingehen. Daraus folgt, dass eine exakte Berechnung der Tangentialkräfte an den einzelnen Zähnen und somit auch der Zahndrehmomente unter ausschließlicher Anwendung der Drehwellentheorie nicht möglich ist.

Soll dennoch eine Ermittlung der Tangentialkräfte an den Zähnen mit Hilfe der Drehwellentheorie erfolgen, so erscheint es naheliegend, die sehr genau berechenbaren Kräfte auf die einzelnen Nuten zu bestimmen, wie dies zum Beispiel von Humburg in [10] beschrieben ist. Dabei entspricht die Nutkraft gerade der Kraft, welche auf einen Leiter im Magnetfeld wirkt, wenn dieser dieselbe elektrische Durchflutung besitzt, wie alle Leiter in der betrachteten Nut. In elektrischen Maschinen mit in Nuten liegenden Leitern wirkt diese Kraft jedoch nicht auf die Leiter selbst, die sich bei genuteten Maschinen im weitgehend feldfreien Raum befinden. Sie wirkt auf die beiden angrenzenden Zähne, beziehungsweise Zahnflanken. In welchem Verhältnis sich die Nutkräfte auf die jeweils benachbarten Zähne aufteilen, lässt sich jedoch nicht einfach bestimmen. Es bietet sich daher zum Beispiel die Annahme an, dass sich die Nutkräfte gleichmäßig auf die angrenzenden Zähne aufteilen, wobei ein einfaches in Abschnitt 5.5 dargestelltes Beispiel zeigt, dass dies physikalisch nicht immer korrekt sein kann. 
Um eine Alternative zur gerade genannten Berechnungsmethode zu bieten, soll nachfolgend ein Verfahren entwickelt werden, welches die realen Vorgänge in den Nuten zumindest näherungsweise einbezieht.

Bei linearer Betrachtung der Maschine lässt sich das Superpositionsprinzip anwenden. Die Induktionen an den Zahnflanken, woraus sich die magnetischen Grenzflächenkräfte ergeben, werden dazu in zwei unterschiedlich wirkende Anteile zerlegt und anschließend überlagert.

Ein Anteil ist das magnetische Feld, das durch die Ströme in den Nuten entsteht, und in Bild 4.2a schematisch dargestellt ist. Überlagert wird dieses mit dem Feldanteil, welcher sich im Nutraum aus einer vorhandenen radialen Luftspaltinduktion ergibt. Ein derartiges Feld ist in Bild 4.2b dargestellt.

Die nachfolgende Herleitung ist insbesondere von der Geometrie der Zahnflanken abhängig. Zur einfacheren theoretischen Durchdringung wird eine geradlinige Flanke gewählt, welche senkrecht zum Luftspalt steht. Wird zudem davon ausgegangen, dass die Nut am Nutschlitz deutlich schmaler ist als am Nutgrund, so genügt es, nur hier ein annähernd tangential gerichtetes Feld zu berücksichtigen. Randeffekte, wie zum Beispiel die Ausdehnung des magnetischen Felds in Richtung der $r$-Achse in der Nutmitte, was in Bild $4.2 \mathrm{a}$ schematisch dargestellt ist, sollen vereinfachend vernachlässigt werden. Inwieweit dies legitim ist, hängt von der jeweiligen Nutgeometrie ab.

Die Induktion im Nutschlitz, welche sich aus der oben erstgenannten Feldquelle, nämlich den Nutströmen $i_{\mathrm{N}}$, ergibt und mit den getroffenen Vereinfachungen in Richtung der $x_{1}$-Koordinate verläuft, lässt sich bei Annahme von $\mu_{\mathrm{Fe}} \rightarrow \infty$ aus dem Ringintegral um eine Nut zu

$$
\oint \mathbf{H} \mathrm{d} \mathbf{s}=\int \mathbf{S d} \mathbf{A} \quad \Leftrightarrow \quad \mathbf{B}_{\mathrm{i}}=B_{\mathrm{i}} \cdot \mathbf{e}_{\mathrm{x}_{1}}=\frac{-\mu_{0} \cdot w_{\mathrm{N}} \cdot i_{\mathrm{N}}}{b_{\mathrm{No}}^{*}}
$$

bestimmen. Dabei ist $b_{\text {No }}^{*}$ die Breite des Nutschlitzes gemäß Bild $4.2 \mathrm{~b}$ und

$$
A_{\mathrm{N}}^{*}=\frac{w_{\mathrm{N}} \cdot i_{\mathrm{N}}}{b_{\mathrm{No}}^{*}}
$$

gerade der Strombelag an der Nut im Längenmaß.

Die Induktion der zweitgenannten Feldquelle entstammt dem in die Nut eintretenden Fluss

$$
\Phi_{\mathrm{r}}(t)=l_{\mathrm{i}} \cdot \int_{x_{(n)}-\frac{b_{\mathrm{No}}}{2}}^{x_{(n)}+\frac{b_{\mathrm{No}}}{2}} B_{\mathrm{r}}\left(x_{1}, t\right) \cdot r \mathrm{~d} x_{1},
$$

welcher sich in der Nut auf den Teilfluss $\Phi_{\mathrm{r} 1}(t)$ aufteilt, der durch die linke, und den Teilfluss $\Phi_{\mathrm{r} 2}(t)$, der durch die rechte Zahnflanke verläuft. Die Richtungsangaben „links“ und „rechts" gelten dabei für die in Bild 4.2 gewählte Ansicht. Daraus ergibt sich an den Zahnflanken die Induktion

$$
B_{\mathrm{rt} 1,2}(r, t)=\frac{\Phi_{\mathrm{r} 1,2}(t)}{l_{\mathrm{i}}} \cdot \vartheta(r)
$$

wobei $\vartheta(r)$ die Verteilung des Flusses über der Höhe der Zahnflanke darstellt. 


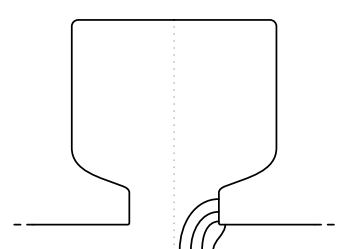

(a) Eintritt in den näherliegenden Zahn

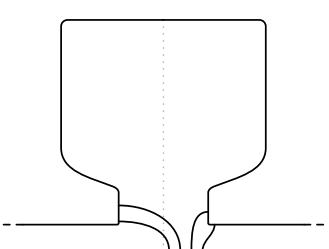

(b) Gleichmäßige Aufteilung auf beide angrenzenden Zähne

Bild 4.3: Skizzierung der möglichen Modellbildungen des Verlaufes eines theoretischen Luftspaltflusses, welcher im Bereich nur einer Nuthälfte auftritt

Da sich der Fluss in zwei verschiedene Richtungen aufteilt, gilt

$$
\mathbf{B}_{\mathrm{rt} 1}(r, t)=-B_{\mathrm{rt} 1}(r, t) \cdot \mathbf{e}_{\mathrm{x}_{1}} \quad \text { und } \quad \mathbf{B}_{\mathrm{rt} 2}(r, t)=+B_{\mathrm{rt} 2}(r, t) \cdot \mathbf{e}_{\mathrm{x}_{1}}
$$

Sind die beiden Zähne an der Nut identisch, so teilt sich eine im Bereich des Nutschlitzes konstante radiale Luftspaltinduktion, wie sie näherungsweise bei allen Induktionswellen mit einer Wellenlänge deutlich größer der Nutschlitzbreite entsteht, gleichmäßig auf beide Zahnflanken auf. Ein derartiger Fall ist in Bild 4.2b dargestellt.

Bei einer nichthomogenen Luftspaltinduktion im Bereich des Nutschlitzes oder ungleichen Zahnflanken wird sich der magnetische Fluss hingegen ungleichmäßig auf die beiden Zahnflanken aufteilen. Die genaue Bestimmung der jeweiligen Flussanteile je Zahnflanke bedarf zum Beispiel einer Finite-Elemente-Berechnung. Vereinfachend soll an dieser Stelle mit einer Näherung gerechnet werden. Dabei drängt sich insbesondere das Modell auf, dass der Luftspaltfluss in den jeweils näherliegenden Zahn eintritt, wie dies in Bild 4.3a skizziert ist. Damit würde

$$
\tilde{\Phi}_{\mathrm{r} 1}(t)=l_{\mathrm{i}} \cdot \int_{x_{(n)}-\frac{b_{\mathrm{No}}}{2}}^{x_{(n)}} B_{\mathrm{r}}\left(x_{1}, t\right) \cdot r \mathrm{~d} x_{1} \quad \text { und } \quad \tilde{\Phi}_{\mathrm{r} 2}(t)=l_{\mathrm{i}} \cdot \int_{x_{(n)}}^{x_{(n)}+\frac{b_{\mathrm{No}}}{2}} B_{\mathrm{r}}\left(x_{1}, t\right) \cdot r \mathrm{~d} x_{1}
$$

gelten. In Abschnitt 5.4 wird sich jedoch zeigen, dass diese Näherung zu einer deutlichen - aber falschen - Überhöhung einer einzelnen Drehmomentkomponente führt, sodass alternativ angenommen wird, dass sich der gesamte Luftspaltfluss im Bereich der Nuten gleichmäßig auf die angrenzenden Zahnflanken aufteilt, wie dies in Bild 4.3b skizziert ist. Es gilt damit

$$
\Phi_{\mathrm{r} 1}(t)=\Phi_{\mathrm{r} 2}(t)=\frac{l_{\mathrm{i}}}{2} \cdot \int_{x_{(n)}-\frac{b_{\mathrm{N} 0}}{2}}^{x_{(n)}+\frac{b_{\mathrm{N} 0}}{2}} B_{\mathrm{r}}\left(x_{1}, t\right) \cdot r \mathrm{~d} x_{1}
$$

Resultierend ergeben sich aus (4.29) und (4.32) die Induktionen an den Zahnflanken zu

$$
B_{\mathrm{t} 1,2}(r, t)=B_{\mathrm{i}}(t) \mp B_{\mathrm{rt} 1,2}(r, t)=-\mu_{0} \cdot A_{\mathrm{N}}^{*}(t) \mp \frac{\Phi_{\mathrm{r} 1,2}(t)}{l_{\mathrm{i}}} \cdot \vartheta(r)
$$


und die daraus resultierenden Zugspannungen mit (4.23) $\mathrm{zu}$

$$
\sigma_{\mathrm{t} 1,2}(t)=\frac{1}{2 \mu_{0}}\left(\mu_{0}^{2} A_{\mathrm{N}}^{* 2}(t) \pm 2 \mu_{0} A_{\mathrm{N}}^{*}(t) \frac{\Phi_{\mathrm{r} 1,2}(t)}{l_{\mathrm{i}}} \cdot \vartheta(r)+\frac{\Phi_{\mathrm{r} 1,2}^{2}(t)}{l_{\mathrm{i}}^{2}} \cdot \vartheta^{2}(r)\right) .
$$

Nach Integration über die Fläche der Zahnflanke ergibt sich daraus die Tangentialkraft, welche sich im betrachteten Fall als

$$
F_{\mathrm{t} 1,2}(t)=\underbrace{\frac{1}{2} l_{\mathrm{i}} h_{\mathrm{No}} \mu_{0} A_{\mathrm{N}}^{* 2}(t)}_{=: F_{\mathrm{t} \alpha 1,2}} \pm \underbrace{A_{\mathrm{N}}^{*}(t) \Phi_{r_{\mathrm{r} 1,2}(t) \int_{r_{1 \mathrm{i}}+h_{\mathrm{No}}}^{=1} \vartheta(r) \mathrm{d} r}}_{=: F_{\mathrm{t} \beta 1,2}}+\underbrace{\frac{\Phi_{\mathrm{r} 1,2}^{2}(t)}{2 \mu_{0} l_{\mathrm{i}}} \int_{r_{1 \mathrm{i}}}^{r_{1 \mathrm{i}}+h_{\mathrm{No}}} \vartheta^{2}(r) \mathrm{d} r}_{=: F_{\mathrm{t} \gamma 1,2}}
$$

darstellen lässt. Dabei muss das Integral der Funktion $\vartheta(r)$ zur Darstellung der Flussverteilung über die Höhe des Nutschlitzes $h_{\text {No }}$ gerade zu 1 werden.

Mit den nun bekannten Kräften auf die einzelnen Zahnflanken ist es sowohl möglich, die Kräfte je Nut als auch die Kräfte auf die einzelnen Zähne zu berechnen. Die Kraft auf eine Nut ist dabei die Differenz der Kräfte auf die beiden angrenzenden Zahnflanken, also

$$
F_{\mathrm{tN}}(t)=l_{\mathrm{i}} \cdot A_{\mathrm{N}}^{*}(t) \cdot \int_{x_{(n)}-\frac{b_{\mathrm{No}(n)}}{2}}^{x_{(n)}+\frac{b_{\mathrm{No}(n)}}{2}} B\left(x_{1}, t\right) \cdot r \mathrm{~d} x_{1}+\frac{\Phi_{\mathrm{r} 1}^{2}(t)-\Phi_{\mathrm{r} 2}^{2}(t)}{2 \mu_{0} l_{\mathrm{i}}} \int_{r_{1 \mathrm{i}}}^{r_{1 \mathrm{i}}+h_{\mathrm{No}}} \vartheta^{2}(r) \mathrm{d} r .
$$

Hierin entspricht der vordere Summand bei Aufsummierung über alle Nuten der Drehmomentgleichung (4.10), sodass der hintere Summand (4.11) widerspiegeln oder zumindest mit diesem Drehmomentanteil verwandt sein muss. Eine Gegenüberstellung beider Drehmomentgleichungen ist in Abschnitt $5.3 \mathrm{zu}$ finden.

Die Kraft auf den Zahn $(n)$ ergibt sich aus der in negativer Richtung angreifenden Kraft $F_{\mathrm{t} 1(n)}(t)$ und der in positiver Richtung angreifenden Kraft $F_{\mathrm{t} 2(n+1)}(t) \mathrm{zu}$

$$
F_{\mathrm{tz}(n)}(t)=F_{\mathrm{t} 2(n+1)}(t)-F_{\mathrm{t} 1(n)}(t) \text {. }
$$

Zur Berechnung des Zahndrehmoments, das wie in Bild 4.1a dargestellt am Zahngrund wirkt, müsste korrekterweise eine Integration des Produkts der Zugspannung an der Zahnflanke mit dem Hebelarm, also dem Abstand zum Zahngrund, über die Zahnhöhe durchgeführt werden. Um ein einfacher handhabbares Ergebnis zu erhalten, wird stattdessen jedoch die Vereinfachung angewandt, die Tangentialkraft, die in der Regel an einem im Verhältnis zur Zahnhöhe nur kleinen Teil angreift, mit der Zahnhöhe als Hebelarm zu multiplizieren, um so das Zahndrehmoment zu erhalten:

$$
M_{\mathrm{t} 1,2}(t)=\int_{-l_{\mathrm{i}} / 2}^{l_{\mathrm{i}} / 2} \int_{r_{1 \mathrm{i}}}^{r_{1 \mathrm{i}}+h_{\mathrm{z}}}\left(r_{1 \mathrm{i}}+h_{\mathrm{z}}-r\right) \cdot \sigma_{\mathrm{t} 1,2}(r, z, t) \mathrm{d} r \mathrm{~d} z \approx F_{\mathrm{t} 1,2}(t) \cdot h_{\mathrm{z}} .
$$

Damit lässt sich das am Jochansatz wirkende Zahndrehmoment als Summe dreier Anteile, wie diese bereits in den Tangentialkräften gemäß (4.38) zu sehen sind, als

$$
M_{\mathrm{tz}(n)}(t) \approx\left[F_{\mathrm{t} \alpha(n)}(t)+F_{\mathrm{t} \beta(n)}(t)+F_{\mathrm{t} \gamma(n)}(t)\right] \cdot h_{\mathrm{z}(n)}=F_{\mathrm{tz}(n)}(t) \cdot h_{\mathrm{z}(n)}
$$


darstellen. Diese drei Anteile sollen nachfolgend zwecks theoretischer Durchdringung einzeln betrachtet werden.

Die Tangentialkraft $F_{\mathrm{t} \alpha(n)}(t)$ ist bei gleichen Nutgeometrien mit gleicher Wicklung direkt proportional zur Differenz der Quadrate der Ströme in benachbarten Nuten und damit unabhängig von der Läufergeometrie. Ihre Frequenz entspricht der doppelten Netzfrequenz. Es gilt:

$$
F_{\mathrm{t} \alpha(n)}(t)=l_{\mathrm{i}} \cdot \frac{h_{\mathrm{No}} \mu_{0}}{2}\left(\frac{w_{(n+1)}^{2} \cdot i_{(n+1)}^{2}(t)}{b_{\mathrm{o}(\mathrm{n}+1)}^{* 2}}-\frac{w_{(n)}^{2} \cdot i_{(n)}^{2}(t)}{b_{\mathrm{o}(\mathrm{n})}^{* 2}}\right) .
$$

Die erfahrungsgemäß störendste, weil größte Komponente der Tangentialkraft

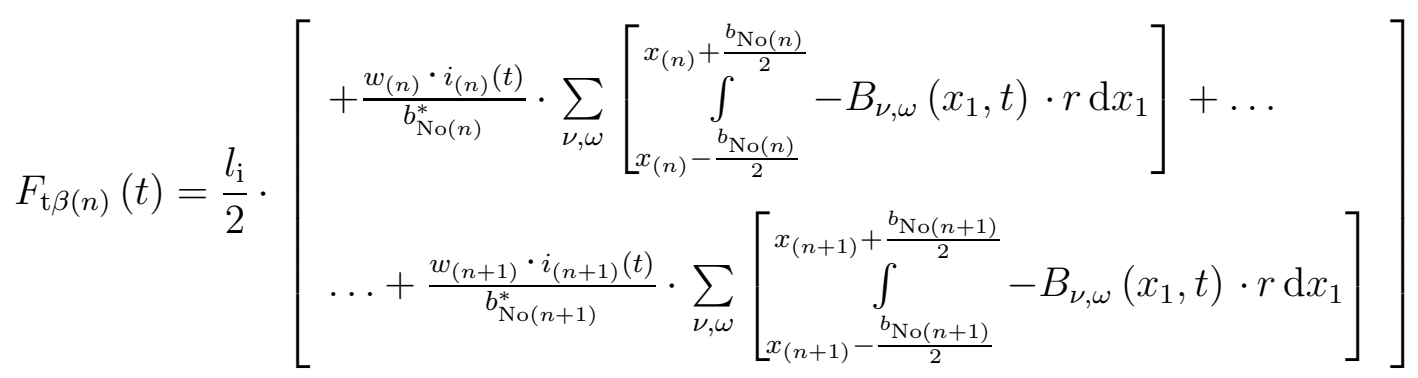

ergibt sich aus den Strömen $i_{(n)}(t)$ und $i_{(n+1)}(t)$ in den zum Zahn $(n)$ benachbarten Nuten sowie der im Bereich des Nutschlitzes vorhandenen Luftspaltinduktion $B\left(x_{1}, t\right)$. Das in (4.44) enthaltene Integral über die Luftspaltinduktion lässt sich dabei zu

$$
\int_{x_{(n)}-\frac{b_{\mathrm{No}(n)}}{2}}^{x_{(n)}+\frac{b_{\mathrm{No}(n)}}{2}}-B_{\nu, \omega}\left(x_{1}, t\right) \cdot r \mathrm{~d} x_{1}=\frac{-2 \hat{B}_{\nu, \omega} \cdot r}{\nu} \cdot \cos \left(\nu x_{(n)}-\omega_{\nu} t+\varphi_{\nu}\right) \sin \left(\nu \frac{b_{\mathrm{No}(n)}}{2}\right)
$$

bestimmen, sodass sich Minima für $\nu=k \cdot \frac{4 \pi}{b_{\mathrm{No}(n)}}$ mit $k=0, \pm 1, \pm 2, \ldots$ ergeben, also für relativ große räumliche Ordnungszahlen. Die Kreisfrequenzen der Kräfte $F_{\mathrm{t} \beta(n)}(t)$, welche sich aus Induktionswellen mit den Kreisfrequenzen $\omega_{\nu}$ ergeben, sind

$$
\omega_{\beta \nu}=\omega_{\nu} \pm \omega_{1}
$$

Für den dritten Teil der Tangentialkräfte, nämlich

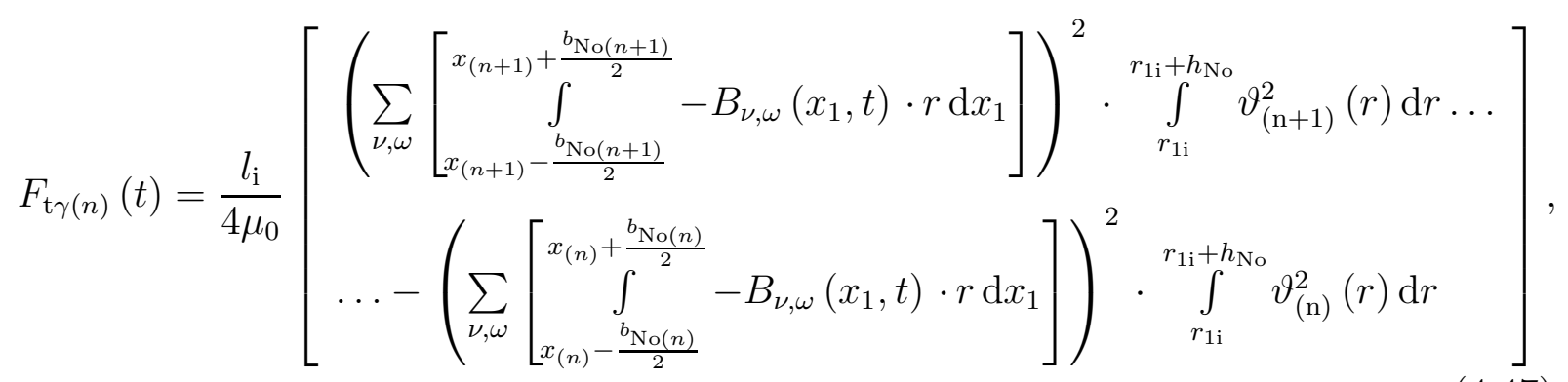

welcher ausschließlich von der Luftspaltinduktion $B\left(x_{1}, t\right)$ abhängt, ergeben sich die Kreisfrequenzen aus den jeweiligen Induktionswellen $\nu$ und $\tilde{\nu} \mathrm{zu}$

$$
\omega_{\gamma \nu, \tilde{\nu}}=\omega_{\nu} \pm \omega_{\tilde{\nu}} .
$$


Die Kreisfrequenzen $\omega_{\gamma \nu, \tilde{\nu}}$ bestehen folglich, wie mit (3.54) und (3.130) zu erkennen, aus einer Kombination eines Vielfachen der Winkelgeschwindigkeit des Läufers $\Omega$ und betragsmäßig maximal dem doppelten der Ständerkreisfrequenz $\omega_{1}$.

Letztendlich ergibt sich ein resultierendes Drehmoment an den Ständerzähnen, welches jeweils zu einem Anteil aus den Radialkräften und einem Anteil aus den Tangentialkräften besteht:

$$
M_{\mathrm{z}(n)}(t)=M_{\mathrm{rz}(n)}(t)+M_{\mathrm{tz}(n)}(t)
$$

Die in (4.43), (4.44) und (4.47) definierten tangentialen Kräfte $F_{\mathrm{t} \alpha(n)}, F_{\mathrm{t} \beta(n)}$ und $F_{\mathrm{t} \gamma(n)}$ enthalten jeweils eine Multiplikation mit der Länge $l_{\mathrm{i}}$ des Aktivteils der Maschine, welche sich aus einer Integration über diese Länge ergibt.

Da $F_{\mathrm{t} \alpha(n)}$ keine ortsabhängigen Größen enthält, bleibt (4.43) auch für den geschrägten Fall gültig. Die Kräfte $F_{\mathrm{t} \beta(n)}$ und $F_{\mathrm{t} \gamma(n)}$ enthalten dagegen eine Integration über die Luftspaltinduktion. Wie in (3.73) ersichtlich, führt die Integration damit zu einer Multiplikation mit dem Schrägungsfaktor $\xi_{\mathrm{schr}}$, der in $F_{\mathrm{t} \gamma(n)}$ sogar quadratisch eingeht. In (4.44) und (4.47) müssen folglich die Amplituden der Induktionswellen mit den jeweiligen Ständerschrägungsfaktoren gemäß (3.75) multipliziert werden.

\subsection{Jochzugwellen}

Insbesondere für den häufigen Fall, dass sich der Ständer mit einer zylinderförmigen Außenkontur unter vollflächigem Kontakt in einem ebenfalls im Wesentlichen zylinderförmigen Gehäuse befindet, welches einen gegenüber dem Ständer nur unwesentlich größeren Außendurchmesser besitzt, spiegelt die Schallemission, welche für den Ständer berechnet wird, gut die Emission des Motors im Gehäuse wider. Aus diesem Grund wird für die in Abschnitt 4.5 gezeigte Berechnung der Schallabstrahlung des Motors ein Modell verwendet, welches im Wesentlichen die Jochzugwellen als Eingangsgrößen verwendet, also die Kräfte, welche direkt am Ständerjoch angreifen.

Die am Joch angreifenden Kräfte setzen sich dabei, wie bereits von Weh [33] und Wachta [32] beschrieben, aus den vom Luftspalt über die Zähne übertragenen Radialkräften und den Zahndrehmomenten zusammen. Die zeitvarianten Fourierkoeffizienten des erstgenannten Anteils ergeben sich dabei aus der Fourierreihenentwicklung der am Joch wirkenden Zahnkräfte gemäß (4.26) über der Winkelkoordinate $x_{1}$ bei Darstellung als zeitvariante Kraftdichte

$$
\sigma_{\mathrm{j}(n)}\left(x_{1}, t\right)= \begin{cases}\frac{F_{\mathrm{z}(n)}(t)}{l_{\mathrm{i}} r_{\mathrm{j}} \tilde{b}_{\mathrm{z}}} & \text { für } x_{(\mathrm{n})}-\frac{b_{\mathrm{z}}}{2} \leq x_{1} \leq x_{(\mathrm{n})}+\frac{b_{\mathrm{z}}}{2} \\ 0 & \text { sonst }\end{cases}
$$

$\mathrm{zu}$

$$
\begin{aligned}
\hat{\sigma}_{\mathrm{jF} \mu \nu}(t)= & \mathrm{j} \cdot \frac{2 r \hat{\sigma}_{\mu \nu}}{\mu k \pi r_{\mathrm{j}} \tilde{b}_{\mathrm{z}}} \cdot \sin \left(\frac{\mu \pi}{N_{1}}\right) \cdot \sin \left(\frac{k \tilde{b}_{\mathrm{z}}}{2}\right) \\
& \cdot \sum_{n=1}^{N_{1}}\left[\mathrm{e}^{\mathrm{j} \frac{(\mu-k)(2 n+1) \pi}{N_{1}}} \cdot \mathrm{e}^{\mathrm{j}\left(-\omega_{\nu} t+\varphi_{\mu \nu}\right)}-\mathrm{e}^{-\mathrm{j} \frac{(\mu+k)(2 n+1) \pi}{N_{1}}} \cdot \mathrm{e}^{-\mathrm{j}\left(-\omega_{\nu} t+\varphi_{\mu \nu}\right)}\right] .
\end{aligned}
$$


Hierbei ist $k$ die räumliche Ordnungszahl der Jochzugwellen, $r_{\mathrm{j}}$ der Radius am Ständernutgrund und $\tilde{b}_{\mathrm{z}}=\tilde{b}_{\mathrm{z}}^{*} / r_{\mathrm{j}}$ die Zahnbreite im Bogenmaß auf Höhe von $r_{\mathrm{j}}$.

Ähnlich wie zur Berechnung der Jochzugwellen aufgrund der radialen Zahnkräfte wird auch zur Berechnung der auf den Zahndrehmomenten basierenden Jochzugwellen eine orts- und zeitabhängige Kraftdichte

$$
\sigma_{\mathrm{j}(n)}\left(x_{1}, t\right)= \begin{cases}\frac{12 M_{\mathrm{z}(n)}(t)}{l_{\mathrm{i}} r_{\mathrm{j}}^{2} \tilde{b}_{z}^{3}} \cdot\left(x_{(n)}-x_{1}\right) & \text { für } x_{(\mathrm{n})}-\frac{b_{\mathrm{z}}}{2} \leq x_{1} \leq x_{(\mathrm{n})}+\frac{b_{\mathrm{z}}}{2} \\ 0 & \text { sonst }\end{cases}
$$

am Angriffspunkt des Zahns am Ständerjoch berechnet, um per räumlicher Fouriertransformation die Jochzugwellen zu ermitteln.

In die Jochzugwellen aufgrund der Zahndrehmomente fließen hierbei, wie in (4.49) zu sehen, sowohl die Tangentialkräfte auf die Ständerzähne, bestehend aus den Anteilen $F_{\mathrm{t} \alpha}$, $F_{\mathrm{t} \beta}$ und $F_{\mathrm{t} \gamma}$ gemäß (4.43), (4.44) und (4.47) als auch die Zahndrehmomente aufgrund der Radialkraftwellen im Luftspalt ein.

Um die Beziehung zwischen den Jochzug- und den Radialkraftwellen im Luftspalt aufgrund der Modulation durch vereinfachend als gleichmäßig angeordnete Ständerzähne aufzeigen zu können, sollen im weiteren Verlauf dieses Abschnitts ausschließlich die Zahndrehmomente gemäß (4.28), welche sich aus den Radialkräften ergeben, beziehungsweise deren Auswirkung genauer betrachtet werden. Aus diesen Drehmomenten resultieren Jochzugwellen mit den Fourierkoeffizienten

$$
\begin{aligned}
& \hat{\sigma}_{\mathrm{jM} \mu \nu}=\frac{12 r^{2} \hat{\sigma}_{\mu \nu}}{\mu r_{\mathrm{j}}^{2} \tilde{b}_{\mathrm{z}}^{3} k \pi} \cdot\left[\frac{2}{\mu} \sin \left(\frac{\mu \pi}{N_{1}}\right)-\frac{2 \pi}{N_{1}} \cos \left(\frac{\mu \pi}{N_{1}}\right)\right] \cdot\left[\frac{2}{k} \sin \left(\frac{k \tilde{b}_{\mathrm{z}}}{2}\right)-\tilde{b}_{\mathrm{z}} \cos \left(\frac{k \tilde{b}_{\mathrm{z}}}{2}\right)\right]
\end{aligned}
$$

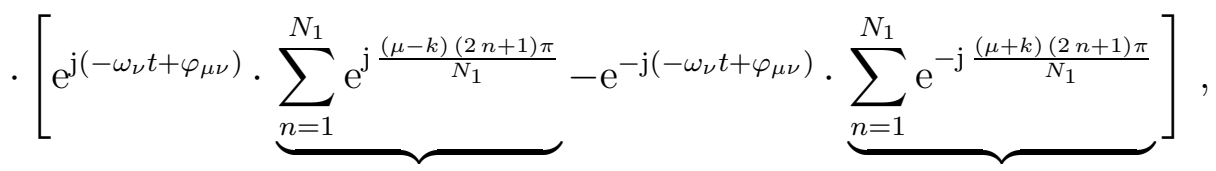

$$
\begin{aligned}
& =\left\{\begin{array}{ll}
N_{1} \mathrm{e}^{\mathrm{j} \varphi} & \text { für }(\mu-k) \in \mathbb{N} \cdot N_{1} \\
0 & \text { sonst }
\end{array}= \begin{cases}N_{1} \mathrm{e}^{\mathrm{j} \varphi} & \text { für }(\mu+k) \in \mathbb{N} \cdot N_{1} \\
0 & \text { sonst }\end{cases} \right.
\end{aligned}
$$

welche eine zu (4.51) sehr ähnliche Struktur aufweisen. Folglich lassen sich die Jochzugwellen, welche sich aus den Radialkraftwellen im Luftspalt ergeben, zu

$$
\sigma_{\mathrm{j}}\left(x_{1}, t\right)=\sum_{\mu, \nu} \hat{\sigma}_{\mathrm{j} \mu \nu} \cos \left(k x_{1}-\omega_{\nu} t+\tilde{\varphi}_{\mu \nu}\right)
$$

zusammenfassen. Dabei gilt

$$
\hat{\sigma}_{\mathrm{j} \mu \nu}=\frac{2 N_{1} r \hat{\sigma}_{\mu \nu}}{\mu k \pi \tilde{b}_{\mathrm{z}}^{*}}\left[\begin{array}{c}
\frac{6 r}{\tilde{b}_{\mathrm{z}}^{2} r_{\mathrm{j}}} \cdot\left[\frac{2}{\mu} \sin \left(\frac{\mu \pi}{N_{1}}\right)-\frac{2 \pi}{N_{1}} \cos \left(\frac{\mu \pi}{N_{1}}\right)\right] \\
\cdot\left[\frac{2}{k} \sin \left(\frac{k \tilde{b}_{\mathrm{z}}}{2}\right)-\tilde{b}_{\mathrm{z}} \cos \left(\frac{k \tilde{b}_{\mathrm{z}}}{2}\right)\right]+\ldots \\
\cdots+\mathrm{j} \cdot \sin \left(\frac{\mu \pi}{N_{1}}\right) \cdot \sin \left(\frac{k \tilde{b}_{\mathrm{z}}}{2}\right)
\end{array}\right] \cdot \mathrm{e}^{\mathrm{j} \varphi}
$$




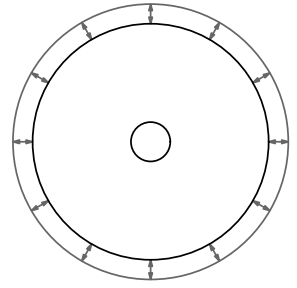

(a) Mode $\nu=0$

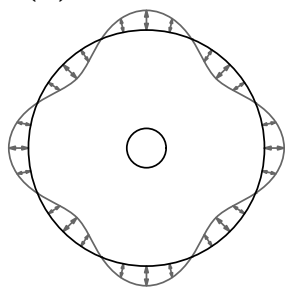

(e) Mode $\nu=4$

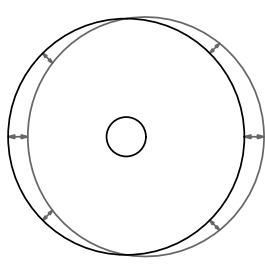

(b) Mode $\nu=1$

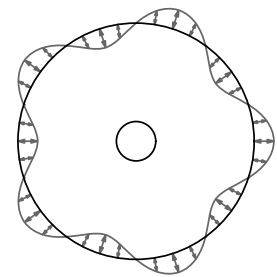

(f) Mode $\nu=5$

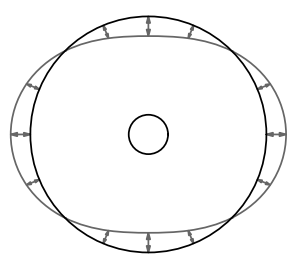

(c) Mode $\nu=2$

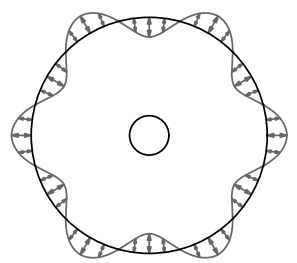

(g) Mode $\nu=6$

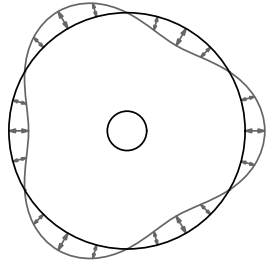

(d) Mode $\nu=3$

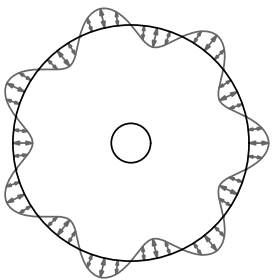

(h) Mode $\nu=7$

Bild 4.4: Beispiele von Eigenformen eines Zylinders

und

$$
\tilde{\varphi}_{\mu \nu}=\varphi_{\mu \nu}+\arg \left\{\sum_{n=1}^{N_{1}} \mathrm{e}^{-\mathrm{j} \frac{(\mu+k)(2 n+1) \pi}{N_{1}}}\right\}+\arg \left\{\hat{\underline{\sigma}}_{\mathrm{j} \mu \nu}\right\} .
$$

Aus der Darstellung der Summen in (4.53) ist erkennbar, dass sich durch die Ständerzähne eine Modulation ergibt. Jede Zugspannungswelle im Luftspalt mit einer räumlichen Ordnungszahl $\mu$ führt am Joch eines gleichmäßig genuteten Ständers zu Kraftwellen der Ordnungszahlen

$$
\kappa=\mu+g N_{1} \text { mit } g=0, \pm 1, \pm 2, \ldots .
$$

Damit wird ersichtlich, dass Radialkraftwellen größerer Ordnungszahl auch zu Jochzugwellen kleiner Ordnungszahl führen. Da gemäß den nachfolgenden Ausführungen in Abschnitt 4.5] ausschließlich Jochzugwellen kleiner Ordnungszahl zu nennenswerten Schallabstrahlungen führen, sind folglich bei gleichmäßig genuteten Ständern alle Radialkraftwellen mit einer räumlichen Ordnungszahl nahe Null oder einem Vielfachen der Ständernutzahl $N_{1}$ zur Berechnung der Jochzugwellen zu berücksichtigen. Die anderen Ordnungszahlen könnten zur Einsparung von Rechenzeit weitgehend vernachlässigt werden.

\subsection{Schwingung des Jochs und Schallabstrahlung}

Ausgehend von den im letzten Abschnitt aufgezeigten Jochzugwellen, kann die Schwingung des Ständerjochs und somit auch die Schallabstrahlung der elektrischen Maschine berechnet werden.

Jede Jochzugwelle besitzt eine räumliche Ordnungszahl $\nu$ und eine Frequenz $f$. Mit der räumlichen Ordnungszahl ist festgelegt, welche Schwingungsform, von denen einige Möglichkeiten in Bild 4.4 dargestellt sind, die jeweilige Kraftwelle anregt. Aus einer mechanischen Berechnung des Ständers ergibt sich für jede einzelne Mode oder Schwingungsbeziehungsweise Eigenform ein frequenzabhängiges Übertragungsverhalten von der anregenden Kraftwelle zur Schwingungsamplitude respektive der abgestrahlten Schallleistung. 
Jordan hat in [11] den Zusammenhang zwischen anregenden Kraftwellen und abgestrahltem Schall dargestellt. Basis der dortigen Berechnungen ist die Betrachtung des Motors, beziehungsweise ausschließlich des Ständers, als Hohlzylinder. Eine weitere Zusammenstellung bezüglich der Biegeschwingungen und der Schallabstrahlung durch Strauß ist in [29] zu finden. Die wesentlichen in [11] und [29] genannten Zusammenhänge werden im Folgenden kurz aufgezeigt:

Jochzugwellen mit der räumlichen Ordnungszahl $\nu=0$ gemäß Bild 4.4a führen nicht zu Biegeschwingungen, wie dies zum Beispiel in Bild 4.4c dargestellt ist, sondern zu einer Stauchung oder Streckung des Jochs in tangentialer Richtung.

Kraftwellen mit $\nu=1$ resultieren in erster Linie in einer Biegeverformung der Welle beziehungsweise des Ständers. Da die meisten Maschinen aufgrund der angestrebten Symmetrie keine signifikanten Kraftwellen mit $\nu=1$ ausbilden, soll auf eine tiefergehende Betrachtung dieser Komponente verzichtet werden, obwohl die in Kapitel (7) vorgestellte Beispielmaschine „B“ gerade aufgrund einer derartigen Kraftwelle ihre dominierende Schallkomponente ausbildet.

Aus den Kraftwellen mit räumlichen Ordnungszahlen $\nu \geq 2$ ergeben sich wellenartige Verformungen des Ständerjochs, wie diese in Bild 4.4c bis Bild 4.4h dargestellt sind. Bei der Frequenz $f_{\nu}=0$ entsteht eine statische Auslenkung des Ständerjochs mit der Amplitude

$$
\Delta \hat{r}_{\mathrm{stat}}=\frac{r_{\mathrm{a} 1} \bar{r}_{1}}{E\left(r_{\mathrm{a} 1}-r_{\mathrm{j} 1}\right)} \cdot \frac{1+3 i^{2}\left(\nu^{2}-1\right)}{i^{2}\left(\nu^{2}-1\right)^{2}} \hat{\sigma}_{\mathrm{j} \nu} .
$$

Dabei sind in (4.58) neben den in Bild 2.1] dargestellten geometrischen Größen der mittlere Radius

$$
\bar{r}_{1}=\frac{r_{\mathrm{j} 1}+r_{\mathrm{a} 1}}{2}
$$

und das Elastizitätsmodul $E$ des Ständerjochs enthalten. Des Weiteren wird in (4.58) die Rückensteife

$$
i=\frac{1}{2 \sqrt{3}} \cdot \frac{r_{\mathrm{a} 1}-r_{\mathrm{j} 1}}{\bar{r}_{1}}
$$

verwendet.

Wird die Auslenkung nicht mehr nur statisch, sondern dynamisch betrachtet, so ergibt sich eine Resonanzüberhöhung mit

$$
\eta_{\nu}=\frac{1}{\sqrt{\left[1-\left(\frac{f}{f_{\mathrm{b} \nu}}\right)^{2}\right]^{2}+4 D^{2}\left(\frac{f}{f_{\mathrm{b} \nu}}\right)^{2}}},
$$

wobei $f_{\mathrm{b} \nu}$ die Eigenfrequenz der Biegeschwingung $\nu$-ter Ordnung und $D$ das Lehr'sche Dämpfungsmaß sind.

Die von jedem einzelnen Punkt der Maschinenoberfläche abgestrahlte Schallleistung ist proportional zur Schnelle der Schwingung, also zum Produkt aus der Amplitude

$$
\hat{y}_{\nu}=\eta_{\nu} \cdot \Delta \hat{r}_{\text {stat }}
$$

und der Frequenz $f_{\nu}$ der jeweiligen Schwingung. Es gilt also

$$
W_{\text {schall } \nu} \sim f_{\nu} \cdot \Delta \hat{r}_{\text {stat }} \cdot \eta_{\nu}
$$




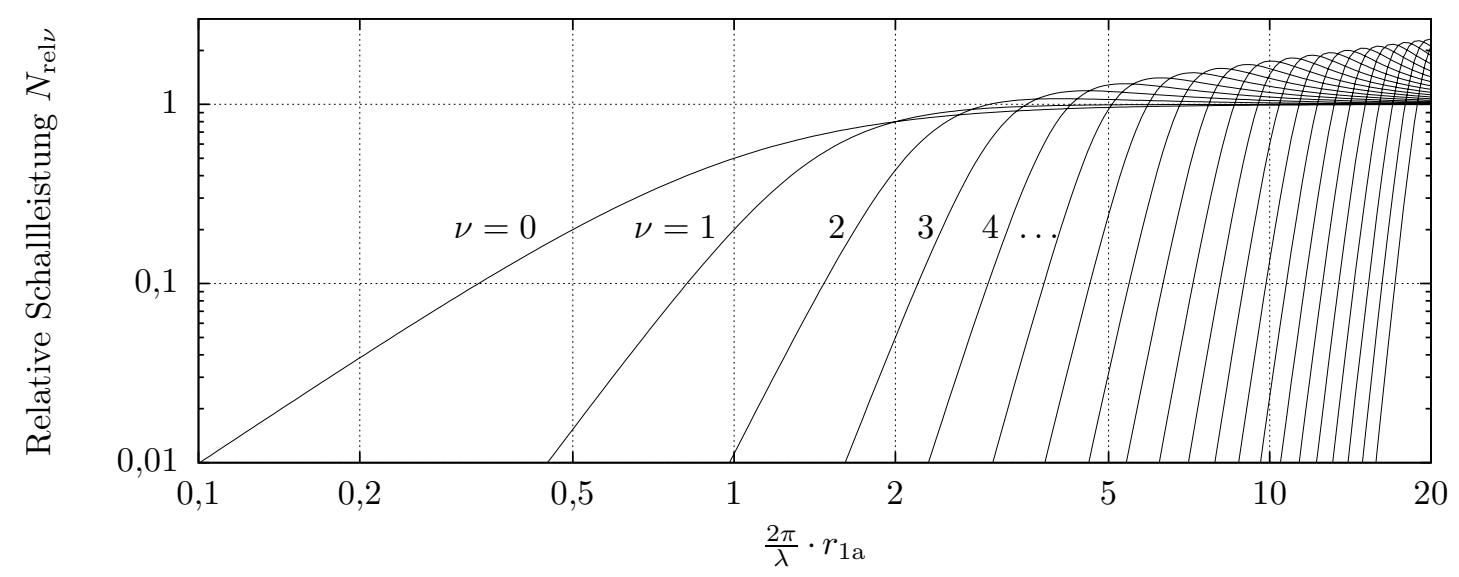

Bild 4.5: Relative Schallleistung eines zonalen Kugelstrahlers

Zur Berechnung der abgestrahlten Schallleistung einer elektrischen Maschine sei diese aus sehr großer Entfernung $l \gg r_{\mathrm{a} 1}$ betrachtet. Die Außenhülle der Maschine wird als Anordnung unendlich vieler Schallquellen modelliert. Bei der Eigenform mit $\nu=0$ führt jede dieser Quellen simultane Schwingungen aus. Eine Phasenverschiebung der Schallwellen im Betrachtungspunkt ist daher für Wellenlängen des Schalls $\lambda=\frac{c}{f}$ deutlich oberhalb des Maschinendurchmessers $D_{\mathrm{a} 1}$ vernachlässigbar. Wird die Wellenlänge $\lambda$ gegenüber $D_{\mathrm{a} 1}$ kürzer, so tritt eine Schallinterferenz am Betrachtungspunkt aufgrund der signifikant unterschiedlichen Entfernungen zu den Quellen auf. Die Maschine wirkt damit leiser.

Für umlaufende Verformungswellen des Ständers mit $\nu>0$ kommt zu der im letzten Absatz genannten Interferenz ein Anteil durch die Phasenverschiebung der einzelnen infinitesimal kleinen Schallquellen hinzu. Zudem ergeben sich sogenannte akustische Kurzschlüsse, also direkte Druckausgleiche zwischen nahe beieinanderliegenden, gegenphasig schwingenden Quellen.

Resultierend ergibt sich die Schallleistung gemäß der Proportionalität nach (4.63) und der multiplikativen Einbeziehung der relativen Schallleistung, wie diese für einzelne Ordnungszahlen in Bild 4.5 dargestellt ist. Es ist zu erkennen, dass Jochzugwellen oberhalb von etwa $\nu=20$ praktisch zu keiner relevanten Schalleistung führen und somit vernachlässigt werden können.

Das hier vorgestellte Modell zur Berechnung der Schallabstrahlung von elektrischen Maschinen berücksichtigt den Ständer sowie das Gehäuse als einfachen Hohlzylinder. Einflüsse durch zum Beispiel Kühlrippen oder andere Elemente am Gehäuse werden folglich nicht berücksichtigt. Insbesondere bezieht das verwendete Modell die Befestigung der Maschine über einen Flansch oder zum Beispiel auf einem Maschinenbett nicht mit ein, wodurch die Ausbildung der verschiedenen Schwingungsformen deutlich beeinflusst werden kann.

Folglich ist die mit Hilfe des vorgestellten Modells berechnete Schallabstrahlung als Näherungslösung anzusehen. 


\subsection{Schlussfolgerung}

In diesem Kapitel wurden verschiedene Wirkmechanismen aufgezeigt, die letztendlich zu Geräuschemissionen führen. Da diese Mechanismen von der Geometrie, wie zum Beispiel der Zahnhöhe und der Zahnkopfbreite abhängen, wäre es denkbar, Geometrien zu finden, bei denen sich verschiedene schwingungsanregende Kräfte gegenseitig aufheben. Dies soll an dieser Stelle jedoch nicht genauer betrachtet werden. Vielmehr soll aufgezeigt werden, welche Frequenzen die einzelnen angeregten Schwingungen und somit auch Töne innehaben.

Ausgehend von einem ideal sinusförmigen Strom der Kreisfrequenz $\omega_{1}$ ergeben sich Ständerfelderregerwellen, die gemäß (3.24) ausschließlich dieselbe Frequenz wie der Strom, nämlich

$$
\omega_{\mathrm{V} 1}=\omega_{\mathrm{A} 1}=\omega_{1}
$$

besitzen. Da die Leitwertwellen bei Vernachlässigung von Sättigungserscheinungen gemäß (3.45) ausschließlich Kreisfrequenzen enthalten, die proportional zur Winkelgeschwindigkeit des Läufers $\Omega$ sind, ergeben sich die Kreisfrequenzen der Ständerinduktionswellen gemäß (3.54) als Linearkombination aus der positiven oder negativen Kreisfrequenz des Ständerstroms $\omega_{1}$ sowie einem positiven oder negativen Vielfachen von $\Omega$. Denselben Wertebereich besitzen die Kreisfrequenzen der Läuferinduktionswellen. Folglich gibt es ausschließlich Induktionswellen der Kreisfrequenzen gemäß

$$
\omega_{\mathrm{B}}=k_{1} \omega_{1}+k_{2} \Omega
$$

mit $k_{2} \in \mathbb{Z}$ und bei Vernachlässigung von Sättigung $k_{1}= \pm 1$, beziehungsweise bei Aufhebung der Einschränkung auf sättigungsfreie Betriebszustände gemäß der Ausführungen in Abschnitt 3.4.2 $k_{1} \in \mathbb{Z}$.

Da sich die geräuschanregenden Radialkraftwellen aus jeweils zwei Induktionswellen, für die (4.65) gilt, bilden und sich auch die Drehmomente aus je maximal zwei Induktionsoder Felderregerwellen beziehungsweise einer Strombelags- und einer Induktionswelle ergeben, deren Kreisfrequenzen sämtlich im Wertebereich von $\omega_{\mathrm{B}}$ liegen, gilt für die Kreisfrequenzen aller Geräuschanregungen

$$
\omega_{\mathrm{G}}=k_{\mathrm{a}} \omega_{1}+k_{\mathrm{b}} \Omega
$$

mit $k_{\mathrm{a}} \in \mathbb{Z}$ und $k_{\mathrm{b}} \in \mathbb{Z}$, wobei sich für den sättigungsfreien Betrieb eine Einschränkung auf $k_{\mathrm{a}} \in\{-2,0,2\}$ ergibt.

Folglich enthält das durch Magnetkräfte hervorgerufene Geräuschspektrum einer beliebigen elektrischen Maschine bei Speisung mit einem einfrequenten harmonischen Strom im hörbaren Bereich nur eine begrenzte Anzahl diskreter Einzeltöne. Ein weißes oder rosa Rauschen kann daher - zumindest bei einfrequentem Strom - nicht erreicht werden. 


\section{Untersuchung des Berechnungsverfahrens}

Die in den vorhergehenden Kapiteln hergeleitete Theorie bietet zur Berechnung einzelner Größen mehrere Alternativen. In diesem Kapitel soll durch Gegenüberstellungen untersucht werden, welche der Alternativen jeweils die geeigneten zur Berechnung der Maschineneigenschaften sind.

\subsection{Vereinfachte Berechnung der resultierenden Luftspaltleitwertwellen}

In Abschnitt 3.4 wird zur Reihenschaltung der Luftspaltleitwertverteilungen, die sich aufgrund der Ständer- beziehungsweise Läufernutung ergeben, ein Verfahren mit einer zweidimensionalen Fourierreihenentwicklung als genaueste Variante vorgestellt. An dieser Stelle soll diesem Verfahren anhand eines Beispiels die vereinfachte Berechnung mittels Multiplikation gemäß (3.42) gegenüber gestellt werden, um die Aussagekraft der vereinfacht berechneten Ergebnisse einschätzen zu können.

Der wesentliche Unterschied beider Verfahren liegt darin, dass bei der multiplikativen Reihenschaltung direkt die mit (3.34) exakt analytisch berechneten Fourierkoeffizienten der ständerseitigen und läuferseitigen Luftspaltleitwertverteilungen genutzt werden können, wohingegen zur Anwendung der zweidimensionalen Fourierreihenentwicklung eine Diskretisierung an einer begrenzten Anzahl von Stützstellen stattfindet. Bei Wahl einer ausreichend hohen Stützstellenzahl, die nachfolgend vorausgesetzt sei, wird dieser Unterschied vernachlässigbar klein und die Reihenschaltung unter Anwendung der zweidimensionalen Fourierreihenentwicklung kann zumindest als mathematisch annähernd exakt betrachtet werden.

Der nachfolgend aufgezeigte Unterschied in den Berechnungsergebnissen beider Verfahren beruht folglich ausschließlich auf der Näherung der beim multiplikativen Verfahren im Nenner stehenden Leitwertverteilungen durch ihre konstanten Anteile, nämlich $\lambda_{10}$ und $\lambda_{20}$. Folglich ist zu erwarten, dass die Ergebnisse beider Verfahren mit abnehmender Nutschlitzbreite, also zunehmender Dominanz von $\lambda_{10}$ in $\lambda_{1}\left(x_{1}\right)$ beziehungsweise $\lambda_{20}$ in $\lambda_{2}\left(x_{1}, \rho\right)$, genauer übereinstimmen.

Um einen quantitativen Eindruck zu geben, sind als Beispiel in Tabelle 5.1 die Luftspaltleitwertwellen der größten Amplituden der in Bild [5.2a dargestellten Maschine mit schmalen Ständernutschlitzen, hier jedoch mit nichtmodulierter Läufernutposition, aufgeführt. Zum Vergleich sind in Tabelle 5.2 die entsprechenden Werte für dieselbe Maschine mit breiten Ständernutschlitzen aufgeführt. Dabei lassen sich die einzelnen Komponenten 
Tabelle 5.1: Luftspaltleitwertwellen; schmale Ständernutschlitze; $n=1495 \mathrm{~min}^{-1}$

\begin{tabular}{rrrr|rrrr}
\hline \multicolumn{3}{c}{ Zweidimensionale FFT } & \multicolumn{4}{|c}{ Multiplikation } \\
$\hat{\lambda}_{n} \cdot \frac{\delta}{\mu_{0}}$ & $\nu$ & $f /[\mathrm{Hz}]$ & $\varphi /\left[^{\circ}\right]$ & $\hat{\lambda}_{n} \cdot \frac{\delta}{\mu_{0}}$ & $\nu$ & $f /[\mathrm{Hz}]$ & $\varphi /\left[^{\circ}\right]$ \\
\hline 0,9110 & 0 & 0,00 & 0,0 & 0,9118 & 0 & 0,00 & 0,0 \\
0,0892 & 96 & 0,00 & 0,0 & 0,0916 & 96 & 0,00 & 0,0 \\
0,0791 & 192 & 0,00 & 180,0 & 0,0812 & 192 & 0,00 & 180,0 \\
0,0764 & 82 & 2043,17 & 0,0 & 0,0788 & 82 & 2043,17 & 0,0 \\
0,0700 & 164 & 4086,33 & 180,0 & 0,0721 & 164 & 4086,33 & 180,0 \\
0,0643 & 288 & 0,00 & 0,0 & 0,0659 & 288 & 0,00 & 0,0 \\
0,0602 & 246 & 6129,50 & 0,0 & 0,0620 & 246 & 6129,50 & 0,0 \\
0,0484 & 328 & 8172,67 & 180,0 & 0,0498 & 328 & 8172,67 & 180,0 \\
0,0473 & 384 & 0,00 & 180,0 & 0,0484 & 384 & 0,00 & 180,0 \\
0,0359 & 410 & 10215,83 & 0,0 & 0,0369 & 410 & 10215,83 & 0,0 \\
0,0306 & 480 & 0,00 & 0,0 & 0,0313 & 480 & 0,00 & 0,0 \\
0,0241 & 492 & 12259,00 & 180,0 & 0,0247 & 492 & 12259,00 & 180,0 \\
0,0165 & 576 & 0,00 & 180,0 & 0,0168 & 576 & 0,00 & 180,0 \\
0,0140 & 574 & 14302,17 & 0,0 & 0,0143 & 574 & 14302,17 & 0,0 \\
0,0063 & 656 & 16345,33 & 180,0 & 0,0064 & 656 & 16345,33 & 180,0 \\
0,0060 & -14 & 2043,17 & 0,0 & 0,0040 & -14 & 2043,17 & 0,0 \\
0,0060 & 178 & 2043,17 & 0,0 & 0,0040 & 178 & 2043,17 & 0,0 \\
0,0055 & 260 & 4086,33 & 180,0 & 0,0036 & 260 & 4086,33 & 180,0 \\
\hline
\end{tabular}

aufgrund ihrer Frequenz weitestgehend dem Ständer, dem Läufer oder einer Überlagerung von beiden zuordnen.

Es zeigt sich bei Betrachtung der beiden Tabellen, dass die Vereinfachung gemäß (3.42) zwar keine exakten, jedoch insbesondere bei schmalen Nutschlitzen für qualitative Aussagen akzeptable Ergebnisse liefert.

\subsection{Vergleich von diskreter und kontinuierlicher Betrachtung des Läufers}

Die kontinuierliche Betrachtung des Läuferkäfigs stellt eine einfache analytische Beschreibungsmöglichkeit dar, deren Genauigkeit an dieser Stelle untersucht werden soll. Da bei der Modellbildung zur kontinuierlichen Betrachtung des Läufers die Einflüsse der einzelnen Läufermaschen und -zähne gemittelt werden, erscheint eine Betrachtung von Effekten mit einer räumlichen Ordnungszahl oberhalb der Läufernutzahl als nicht zielführend. Vielmehr ist zu erwarten, dass innerhalb einer Periodenweite der untersuchten Wellen des Ringstromes bereits mehrere Läufernuten beziehungsweise Käfigstäbe liegen sollten, um aussagekräftige Ergebnisse zu erhalten.

Aufgrund dieser Einschränkung der Gültigkeit des kontinuierlichen Modells zeigen die nachfolgenden Berechnungsergebnisse ausschließlich die Rückwirkung auf die Induktionshauptwelle. Es sei in diesem Zusammenhang zudem auf das Nyquist-Shannonsche Abtast- 
Tabelle 5.2: Luftspaltleitwertwellen; breite Ständernutschlitze; $n=1495$ min $^{-1}$

\begin{tabular}{rrrr|rrrr}
\hline \multicolumn{3}{c}{ Zweidimensionale FFT } & \multicolumn{4}{|c}{ Multiplikation } \\
$\hat{\lambda}_{n} \cdot \frac{\delta}{\mu_{0}}$ & $\nu$ & $f /[\mathrm{Hz}]$ & $\varphi /\left[^{\circ}\right]$ & $\hat{\lambda}_{n} \cdot \frac{\delta}{\mu_{0}}$ & $\nu$ & $f /[\mathrm{Hz}]$ & $\varphi /\left[^{\circ}\right]$ \\
\hline 0,6473 & 0 & 0,00 & 0,0 & 0,6423 & 0 & 0,00 & 0,0 \\
0,4360 & 96 & 0,00 & 0,0 & 0,4253 & 96 & 0,00 & 0,0 \\
0,0724 & 192 & 0,00 & 180,0 & 0,0725 & 192 & 0,00 & 180,0 \\
0,0666 & 288 & 0,00 & 180,0 & 0,0638 & 288 & 0,00 & 180,0 \\
0,0559 & 82 & 2043,17 & 0,0 & 0,0470 & 82 & 2043,17 & 0,0 \\
0,0512 & 164 & 4086,33 & 180,0 & 0,0431 & 164 & 4086,33 & 180,0 \\
0,0440 & 246 & 6129,50 & 0,0 & 0,0372 & 246 & 6129,50 & 0,0 \\
0,0354 & 328 & 8172,67 & 180,0 & 0,0299 & 328 & 8172,67 & 180,0 \\
0,0262 & 410 & 10215,83 & 0,0 & 0,0223 & 410 & 10215,83 & 0,0 \\
0,0188 & 178 & 2043,17 & 0,0 & 0,0230 & 178 & 2043,17 & 0,0 \\
0,0188 & -14 & 2043,17 & 0,0 & 0,0230 & -14 & 2043,17 & 0,0 \\
0,0179 & 480 & 0,00 & 0,0 & 0,0170 & 480 & 0,00 & 0,0 \\
0,0175 & 492 & 12259,00 & 180,0 & 0,0151 & 492 & 12259,00 & 180,0 \\
0,0172 & 260 & 4086,33 & 180,0 & 0,0210 & 260 & 4086,33 & 180,0 \\
0,0172 & 68 & 4086,33 & 180,0 & 0,0210 & 68 & 4086,33 & 180,0 \\
0,0148 & 342 & 6129,50 & 0,0 & 0,0181 & 342 & 6129,50 & 0,0 \\
0,0148 & 150 & 6129,50 & 0,0 & 0,0181 & 150 & 6129,50 & 0,0 \\
0,0135 & 384 & 0,00 & 0,0 & 0,0136 & 384 & 0,00 & 0,0 \\
\hline
\end{tabular}

theorem verwiesen [22], woraus sich im Sinne eines zweckmäßigen Vergleichs der Läufermodelle die Beschränkung der räumlichen Ordnungszahlen $\mu$ der anregenden Induktionswellen auf $|\mu| \leq k$ bei $2 k+1$ Stützstellen, wie sie zum Beispiel die $N_{2}$ Läufernuten bilden, ergibt.

Beispielhaft sind in Tabelle 5.3 die läuferseitigen Felderregerwellen als Rückwirkung auf die Ständerinduktionshauptwelle für die Maschine gemäß Bild 5.1 dargestellt. Es zeigt sich für die Felderregerwelle der Ordnungszahl $\nu=2$ eine relativ gute Übereinstimmung, während die Nutharmonischen der Läuferwicklung vom kontinuierlichen Modell nicht berücksichtigt werden. Letzteres war dabei zu erwarten, da die in Abschnitt 3.6.6 vorgeschlagene Diskretisierung des kontinuierlichen Modells zur Berücksichtigung der diskreten Nutpositionen bei der Feldbildung nicht angewandt wurde.

Neben der Berechnung gleichmäßig genuteter Maschinen bietet die kontinuierliche Betrachtung des Läufers eine sinnvolle Basis, um die Läuferrückwirkung bei einfach mathematisch beschreibbaren Modulationen der Nutpositionen oder Nutformen, wie zum Beispiel bei einer sinusförmigen Nutanordnung mit unveränderter Läufernutgeometrie, wie sie in Bild 5.2a zu sehen ist, zu berechnen. Das Ergebnis einer derartigen Berechnung ist in Tabelle 5.4 gezeigt, wobei die $b_{2}=6$ Perioden der Modulation sowohl bei der diskreten, als auch bei der kontinuierlichen Berechnung entsprechend (3.71) deutlich in Erscheinung treten.

Die kontinuierliche Darstellung des Läufers nähert den Käfig mit unendlich vielen Stäben an. Daraus folgt, dass die Nachrechnung einer Maschine mit sehr vielen Nuten eine be- 


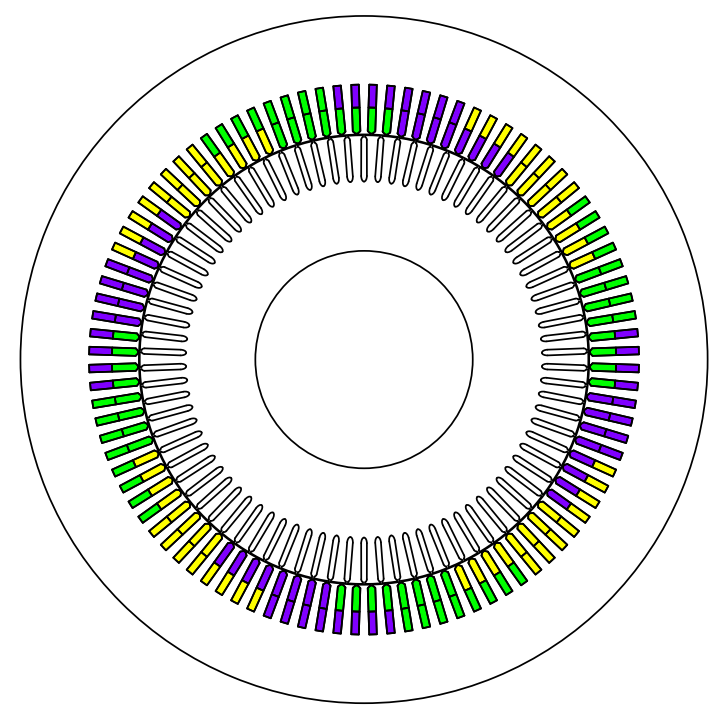

Bild 5.1: Blechschnitt einer Maschine mit $N_{1}=96$ Ständer- und $N_{2}=82$ gleichmäßig angeordneten Läufernuten

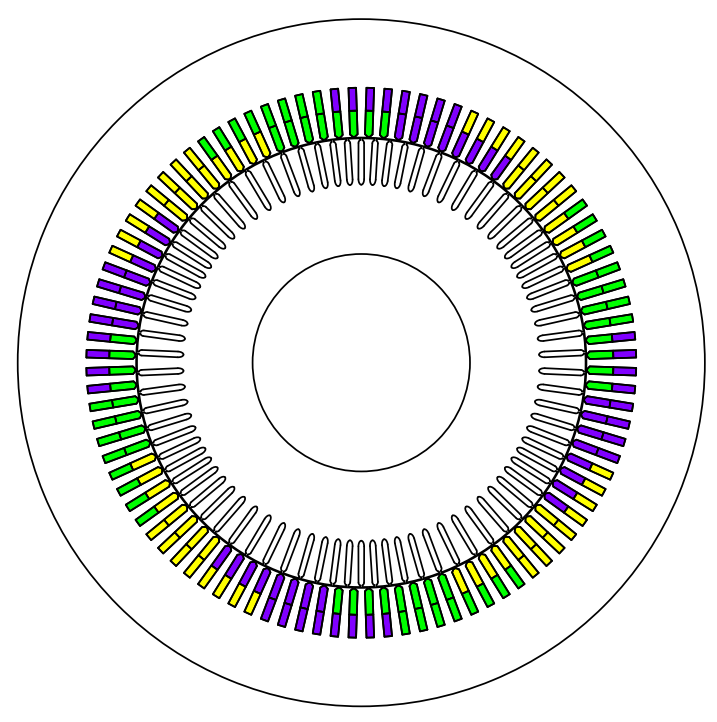

(a) Ohne Anpassung der Läufernutbreiten

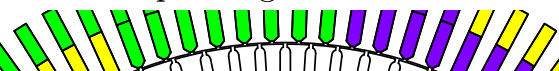
(1)

(c) Ausschnittvergrößerung von $5.2 \mathrm{a}$

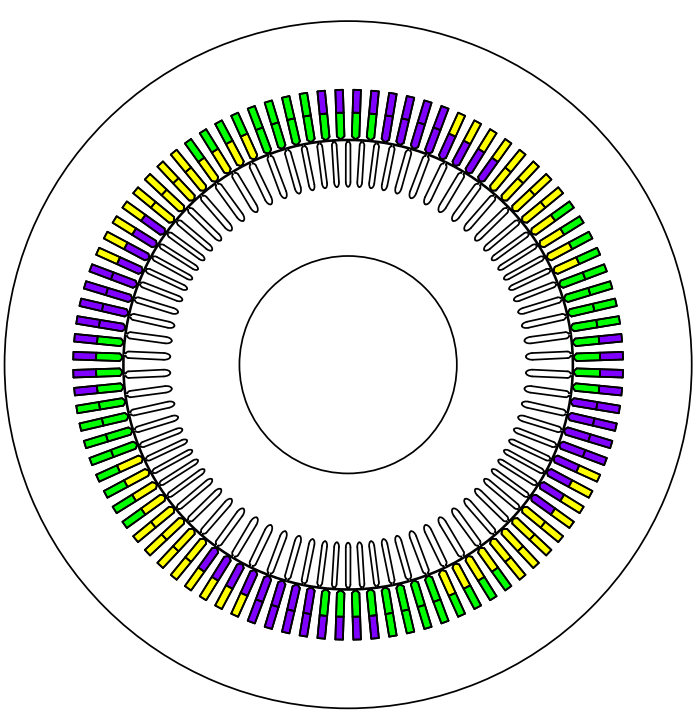

(b) Mit Anpassung der Läufernutbreiten

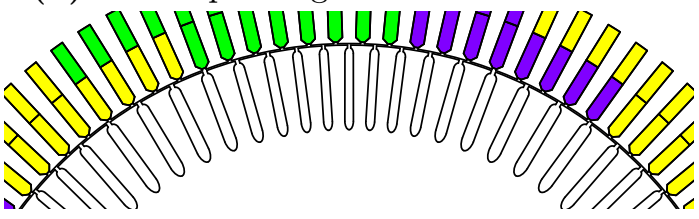

(d) Ausschnittvergrößerung von $5.2 \mathrm{~b}$

Bild 5.2: Blechschnitt einer Maschine mit $N_{1}=96$ Ständer- und $N_{2}=82$ Läufernuten, welche mit $b_{2}=6$ Perioden sinusförmig angeordnet sind. 
Tabelle 5.3: Läuferfelderregerwellen bei $N_{2}=82$ gleichmäßig angeordneten Nuten gemäß Bild 5.1 als Rückwirkung auf die Ständerinduktionshauptwelle

\begin{tabular}{rrrrr|rr}
\hline \multicolumn{6}{c|}{ Diskret } & \multicolumn{2}{|c}{ Kontinuierlich } \\
$\hat{v} /[\mathrm{Am}]$ & $\nu$ & $\nu / p$ & $f /[\mathrm{Hz}]$ & $\varphi /\left[^{\circ}\right]$ & $\hat{v} /[\mathrm{Am}]$ & $\varphi /\left[^{\circ}\right]$ \\
\hline 4820,3 & 2 & 1 & 50,00 & 162,6 & 4823,9 & 162,5 \\
120,5 & 80 & 40 & 1993,17 & $-162,6$ & & \\
114,8 & 84 & 42 & 2093,17 & $-17,4$ & & \\
59,5 & 162 & 81 & 4036,33 & 17,4 & & \\
58,1 & 166 & 83 & 4136,33 & 162,6 & & \\
\hline
\end{tabular}

Tabelle 5.4: Läuferfelderregerwellen bei $N_{2}=82$ in $b_{2}=6$ Perioden sinusförmig angeordneten Nuten gleicher Geometrie gemäß Bild 5.2 als Rückwirkung auf die Ständerinduktionshauptwelle

\begin{tabular}{|c|c|c|c|c|c|c|}
\hline \multicolumn{5}{|c|}{ Diskret } & \multicolumn{2}{|c|}{ Kontinuierlich } \\
\hline$\hat{v} /[\mathrm{Am}]$ & $\nu$ & $\nu / p$ & $f /[\mathrm{Hz}]$ & $\varphi /\left[^{0}\right]$ & $\hat{v} /[\mathrm{Am}]$ & $\varphi /\left[^{\circ}\right]$ \\
\hline 4811,2 & 2 & 1 & 50,00 & 162,5 & 4816,5 & 162,5 \\
\hline 167,8 & 4 & 2 & 99,50 & 149,2 & 161,6 & 150,1 \\
\hline 93,2 & 8 & 4 & 199,50 & $-6,1$ & 88,0 & $-0,7$ \\
\hline 72,2 & 74 & 37 & 1843,67 & $-160,2$ & & \\
\hline 66,7 & 78 & 39 & 1943,67 & $-18,2$ & & \\
\hline 56,6 & 68 & 34 & 1694,17 & $-160,2$ & & \\
\hline 55,3 & 72 & 36 & 1794,17 & $-21,9$ & & \\
\hline 49,2 & 86 & 43 & 2142,67 & 17,3 & & \\
\hline 48,2 & 92 & 46 & 2292,17 & $-165,7$ & & \\
\hline 46,5 & 96 & 48 & 2392,17 & $-14,9$ & & \\
\hline
\end{tabular}




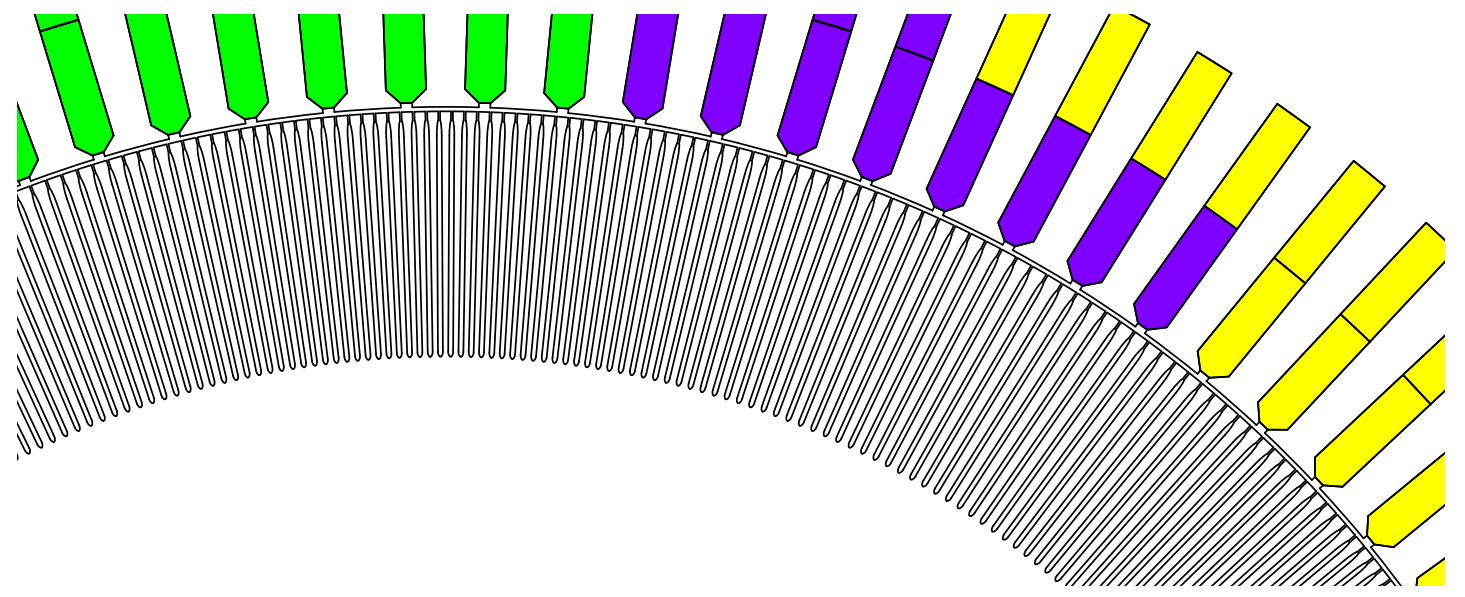

Bild 5.3: Blechschnitt mit $N_{2}=492$ in 6 Blöcken sinusförmig angeordneten Läufernuten

Tabelle 5.5: Läuferfelderregerwellen bei $N_{2}=492$ in $b_{2}=6$ Perioden sinusförmig angeordneten Nuten gleicher Geometrie gemäß Bild 5.3 als Rückwirkung auf die Ständerinduktionshauptwelle

\begin{tabular}{rrrrr|rr}
\hline \multicolumn{5}{c|}{ Diskret } & \multicolumn{2}{|c}{ Kontinuierlich } \\
$\hat{v} /[\mathrm{Am}]$ & $\nu$ & $\nu / p$ & $f /[\mathrm{Hz}]$ & $\varphi /\left[^{\circ}\right]$ & $\hat{v} /[\mathrm{Am}]$ & $\varphi /\left[^{\circ}\right]$ \\
\hline 5048,5 & 2 & 1 & 50,00 & 173,6 & 5048,6 & 173,6 \\
51,9 & 4 & 2 & 99,50 & 107,9 & 51,9 & 105,6 \\
\hline
\end{tabular}

sonders gute Übereinstimmung zwischen diskreter und kontinuierlicher Rechnung zeigen muss. Das kontinuierliche Modell lässt sich damit verifizieren. Zu diesem Zweck ist die Nachrechnung der Maschine gemäß Bild 5.3. welche mit $N_{2}=492$ Läufernuten ausgeführt ist, in Tabelle 5.5 aufgezeigt. Es zeigen sich dabei Abweichungen zwischen den Ergebnissen der Berechnungsmethoden, welche unterhalb von einem Prozent liegen, sodass bei der nach wie vor endlichen Läufernutzahl von einer sehr guten Übereinstimmung gesprochen werden kann.

Zusammenfassend lässt sich erkennen, dass die kontinuierliche Darstellung des Läufers für Effekte mit räumlichen Ordnungszahlen weit unterhalb der Läufernutzahl aussagekräftige Ergebnisse liefert und folglich ein zu deren Untersuchung geeignetes Mittel darstellt.

\subsection{Methoden zur Drehmomentberechnung}

In den Abschnitten 4.1 und 4.3 werden zwei unterschiedliche Ansätze zur Berechnung des Drehmoments von elektrischen Maschinen gezeigt. Dies ist zum einen ein Ansatz, welcher $\mathrm{zu}$ den Gleichungen (4.12) und (4.13) führt und eine direkte Berechnung mit Hilfe der Strombelags-, Felderreger-, Induktions- und Luftspaltleitwertwellen darstellt. Zum Anderen ist dies die Berechnung des Drehmomentes mit (4.39) aus den Tangentialkräften an den Ständerzähnen, was eine abschnittsweise Integration und Summenbildung erfordert.

Ein Vergleich von (4.12) mit dem vorderen Summanden von (4.39) zeigt dabei Überein- 
stimmung. Es gilt also

$$
\underbrace{-\frac{D^{2}}{4} l_{\mathrm{i}} \int_{0}^{2 \pi} A_{1}^{*}\left(x_{1}, t\right) B\left(x_{1}, t\right) \mathrm{d} x}_{=M_{1}^{*}(t)}=\frac{D}{2} \cdot \sum_{n=1}^{n=N_{1}} \underbrace{l_{\mathrm{i}} \cdot b_{\mathrm{No}(n)}^{*} \cdot A_{(n)}^{*}(t) \cdot\left(-B_{\mathrm{r}(n)}(t)\right)}_{=F_{\mathrm{t} \beta \mathrm{N}}(t)} .
$$

Zwischen (4.13) und dem zweiten Summanden von (4.39) ist hingegen Ähnlichkeit, jedoch keine Gleichheit zu erkennen:

$$
\underbrace{\frac{D l_{\mathrm{i}}}{4} \int_{0}^{2 \pi} \frac{\mathrm{d} \lambda\left(x_{2}+\rho_{0}+\Omega t, \rho\right)}{\mathrm{d} \rho_{0}} \cdot V^{2}\left(x_{1}, t\right) \mathrm{d} x_{1}}_{M_{2}^{*}(t)} \neq \frac{D}{2} \sum_{n=1}^{n=N_{1}} \underbrace{\frac{\Phi_{\mathrm{r} 1(n)}^{2}(t)-\Phi_{\mathrm{r} 2(n)}^{2}(t)}{2 \mu_{0} l_{\mathrm{i}}} \int_{r_{1 \mathrm{i}}}^{r_{1 \mathrm{i}}+h_{\mathrm{No}}} \vartheta^{2}(r) \mathrm{d} r}_{=F_{\mathrm{t} \gamma(n)}(t)}
$$

Diese Ungleichheit lässt sich durch die unterschiedlichen Modellbildungen, aus denen die Gleichungen (4.13) und (4.39) hervorgehen, begründen. Die in Abschnitt 4.1 dargestellte Herleitung von (4.13) berücksichtigt ausschließlich die Effekte im Luftspalt wohingegen die Herleitung von (4.39) in Abschnitt 4.3 mit Hilfe der Funktion $\vartheta_{\mathrm{n}}(r)$ auch die Feldverteilung in den Nuten einbezieht. Je nach Genauigkeit der Verteilungsfunktion $\vartheta_{\mathrm{n}}(r)$ lässt sich daher vermuten, dass der zweite Ansatz genauere Ergebnisse liefert.

\subsection{Flussaufteilung zur Berechnung der Tangentialkräfte an den Zähnen}

Anhand des Beispiels einer dreisträngigen zweipoligen Induktionsmaschine mit $N_{1}=6$ Ständernuten, also $q_{1}=1$ Nut je Pol und Strang, soll aufgezeigt werden, weshalb die mit (4.34) getroffene Annahme, dass der Fluss im Bereich einer Nut jeweils in den näher liegenden Zahn eintritt, nicht zielführend ist.

Die Ständerwicklung der oben genannten Maschine erregt neben der Induktionshauptwelle mit $\nu=1$ unter anderem die beiden nutharmonischen Oberwellen der räumlichen Ordnungszahlen $\nu=-5$ sowie $\nu=7$. Vereinfachend sei für das vom Läufer erregte Feld angenommen, dass dieses rein sinusförmig mit $\nu=1$ ist und gegenüber der Ständerinduktionswelle eine Phasenverschiebung um einen Winkel aufweist, der kein Vielfaches von $\pi / 2$ ist.

Um die Problematik der Flussaufteilung aufzuzeigen, soll die Tangentialkraftkomponente $F_{\mathrm{t} \gamma}$ gemäß (4.47), jedoch mit der Annahme, dass der Fluss entsprechend (4.34) jeweils in den nähergelegenen Zahn eintritt, betrachtet werden. In diesem Fall müssen zur Berechnung von $F_{\mathrm{t} \gamma}$ die Induktionen sowohl über die rechten als auch die linken Nuthälften integriert werden. Sind sämtliche Nuten gleich breit, so ergibt sich für eine einzelne Induktionswelle in jeder Nuthälfte dieselbe Amplitude, aber in der Regel unterschiedliche Phasenwinkel.

Werden diese Phasenwinkel mit $\varphi_{\mathrm{r} \nu(n)}$ für den Teil des Flusses der Induktionswelle mit der räumlichen Ordnungszahl $\nu$ durch die rechte Hälfte von Nut $(n)$ beziehungsweise 
$\varphi_{1 \nu(n)}$ für den Teil des Flusses derselben Induktionswelle durch die linke Hälfte von Nut $(n)$ bezeichnet, so ergeben sich für die Induktionshauptwelle sowie alle Nutharmonischen unabhängig von $n$ die konstanten Differenzen der Phasenwinkel

$$
\begin{array}{rrrrr}
\varphi_{\mathrm{r}, 1(n)}-\varphi_{\mathrm{r},-5(n)}=k_{\mathrm{r}, 1,-5} & \text { und } & \varphi_{\mathrm{l}, 1(n)}-\varphi_{\mathrm{l},-5(n)} & =k_{\mathrm{l}, 1,-5} \\
\text { sowie } & \varphi_{\mathrm{r}, 1(n)}-\varphi_{\mathrm{r}, 7(n)}=k_{\mathrm{r}, 1,7} & \text { und } & \varphi_{\mathrm{l}, 1(n)}-\varphi_{\mathrm{l}, 7(n)} & =k_{1,1,7} .
\end{array}
$$

Alle genannten Induktionswellen besitzen dieselbe Kreisfrequenz, nämlich $\omega_{1}$. Folglich entstehen bei der Multiplikation der Feldwellen, welche sich aus der Quadrierung der Fourierreihen in (4.47) ergibt, zeitlich konstante Anteile, die zum Beispiel für die Feldwellen mit $\nu=1$ und $\nu=-5$ aufgrund der identischen Winkeldifferenzen in allen rechten Nuthälften gleich sind. Für alle linken Nuthälften ergeben sich ebenfalls gleiche konstante Anteile, die jedoch nicht denen der rechten Nuthälften entsprechen. Folglich führt die Berechnung jeweils an den rechten Zahnflanken zu einer anderen konstanten Kraft als an den linken Zahnflanken, was sich als konstanter Drehmomentanteil darstellt.

Da dieser konstante Drehmomentanteil, welcher aus Wechselwirkungen je zweier Induktionswellen verschiedener Ordnungzahlen entsteht, bei der Annahme, dass der Fluss in den jeweils näherliegenden Zahn eintritt, in einigen Beispielen das Drehmoment aus Induktionshauptwelle und Ständerstrombelagshauptwelle übertrifft und sich dies in keiner Weise durch numerische Simulationen bestätigen lässt, kann der hier gezeigt Effekt in der Realität nur schwach ausgeprägt vorhanden sein. Aus diesem Grund wurde die gleichmäßige Flussaufteilung gemäß (4.35), bei der das hier aufgezeigte Drehmoment aufgrund der gleichen Phasenwinkel für die jeweils linken und rechten Nuthälften nicht auftaucht, zur Berechnung der Maschinen gewählt. Dies wurde gewählt, obwohl eine gewichtete Überlagerung beider Flussaufteilungen, deren Wichtungsfaktoren zum Beispiel auf Finite-ElementeBerechnungen basieren könnten, die genauesten Ergebnisse liefern müsste.

\subsection{Berechnung der Tangentialkräfte an den Zähnen}

Mit dem Vorliegen der Drehmomentgleichungen (4.12) und (4.13) aus Abschnitt 4.1 liegt es nahe, die Tangentialkräfte an den Ständerzähnen durch die abschnittsweise Integration

$$
\tilde{F}_{\mathrm{tz}}(n)=\frac{l_{\mathrm{i}}}{2} \int_{x_{1(n)}-\frac{b_{\mathrm{Z}}}{2}}^{x_{1(n)}+\frac{b_{\mathrm{Z}}}{2}} \frac{\mathrm{d} \lambda\left(x_{2}+\rho_{0}+\Omega t, \rho\right)}{\mathrm{d} \rho_{0}} \cdot V^{2}\left(x_{1}, t\right)-D \cdot A_{1}^{*}\left(x_{1}, t\right) \cdot B\left(x_{1}, t\right) \mathrm{d} x_{1}
$$

zu bestimmen. Alternativ zeigt Abschnitt 4.3 ein Berechnungsverfahren, welches die Tangentialkräfte aus drei Einzelkomponenten, nämlich $F_{\mathrm{t} \alpha(n)}, F_{\mathrm{t} \beta(n)}$ und $F_{\mathrm{t} \gamma(n)}$ bestimmt, die direkt als Tangentialkräfte an den Zähnen hergeleitet wurden.

Analog zu den Ausführungen bezüglich des Drehmoments in Abschnitt [5.3 ergeben sich aufgrund derselben Unterschiede der Modellierungen der Kraftwirkungen bei den Tangentialkräften dieselben modellbedingten Unterschiede wie bei den Drehmomenten. Zusätzlich zeigt sich der signifikante Unterschied, dass ein Äquivalent von $F_{\mathrm{t} \alpha(n)}$ in (5.5) nicht enthalten ist und dieser Anteil somit auch nicht berücksichtigt wird. 
Eine weitere Diskrepanz zwischen den Berechnungsmethoden zeigt ein einfaches Beispiel, bei dem die Luftspaltinduktion unterhalb der betrachteten Zähne und Nuten konstant sei. Fließst nun in Nut $(n)$ der Strom $i_{(n)}=\hat{i}$ und kein Strom in den Nuten $(n-1)$ und $(n+1)$, gelte also $i_{(n-1)}=i_{(n+1)}=0$, so ergibt sich ein Kraftanteil in Nut $(n)$, nicht aber in den Nuten $(n-1)$ und $(n+1)$. Aufgrund der gleichmäßigen Aufteilung der Nutkräfte auf die angrenzenden Zähne gemäß (5.5) folgt daraus für die Zähne $(n-1)$ und $(n)$ dieselbe Tangentialkraft mit derselben Orientierung. Es handelt sich folglich bei einem Zahn um eine anziehende Kraft und bei dem anderen Zahn um eine abstoßende Kraft, was physikalisch nicht möglich ist.

Mit dem in Abschnitt 4.3 entwickelten Verfahren ergeben sich für das Beispiel aus dem letzten Absatz hingegen jeweils anziehende Kräfte an den Zahnflanken der Zähne $(n-1)$ und $(n)$ in Richtung der stromführenden Nut $(n)$. Die Differenz der Kräfte auf beide Zahnflanken ist dabei für beide Verfahren gleich, sodass sich derselbe Beitrag zum Drehmoment ergibt.

Dieses einfache Beispiel zeigt, dass das in Abschnitt 4.3 entwickelte Verfahren zur Berechnung der Tangentialkräfte an den Zähnen der Berechnung aus den Drehmomentgleichungen vorzuziehen ist, wobei für beide Verfahren abschnittsweise Integrationen mit vergleichbarem Rechenaufwand durchzuführen sind. 


\section{Variation der Ständergeometrie}

Bis hierher wurde der Fokus bei den angestellten Untersuchungen und entwickelten Berechnungsverfahren primär auf die Modulation der Nutpositionen eines Käfigläufers gerichtet. Es liegt jedoch nahe, in die Untersuchung der Varianz der Läufergeometrie auch die Ständergeometrie einzubeziehen.

In diesem Kapitel wird dazu die gezielte Anpassung der Ständergeometrie betrachtet. Die Ergebnisse lassen sich jedoch teilweise auch auf einen Schleifringläufer übertragen, da dieser, genau wie der Ständer, über eine symmetrische Drehstromwicklung verfügt.

Der Aufbau des Ständers mit unterschiedlichen Nutabständen und eventuell auch Querschnitten stellt gegenüber einem gegossenen Käfigläufer höhere Anforderungen an die Fertigung. Insbesondere bei größeren Maschinen, bei denen in der Regel Ganzformspulen eingesetzt werden [31], ist es folglich naheliegend, für alle Spulen dieselbe Spulenweite und dieselben Spulenseitenabmessungen zu nutzen oder sich zumindest auf wenige unterschiedliche zu beschränken. Diese Anforderungen werden im Folgenden berücksichtigt.

\subsection{Gleichmäßige Ständergeometrie}

Vereinfachend wird zunächst eine Ganzlochwicklung mit gleichmäßig angeordneten Spulenseiten und mehreren Nuten je Pol und Strang, also $q_{1}>1$, betrachtet. Im Bereich kleinerer magnetischer Feldstärken wird von unendlich hoher magnetischer Leitfähigkeit des Eisens, also $\mu_{\mathrm{Fe}} \rightarrow \infty$, ausgegangen. Bei höheren magnetischen Feldstärken soll hingegen Sättigung, das heißt eine endliche magnetische Permeabilität des Eisens, in Erscheinung treten. Der Läufer wird zunächst als idealer magnetischer Rückschluss angenommen, welcher selbst keine Induktionswelle erregt.

Ausgehend von der Induktionshauptwelle ergibt sich in erster Linie die bereits in Abschnitt 3.4.2 erwähnte Sättigungswelle, welche durch die Leitwertwelle

$$
\lambda_{\text {sat } 2 p}\left(x_{1}, t\right)=\hat{\lambda}_{\text {sat } 2 p} \cdot \cos \left(2 p x_{1}-2 \omega_{1} t+\varphi+\pi\right)
$$

repräsentiert werden kann. Zusätzlich entstünden bei unterschiedlich breiten Ständerzähnen und damit möglicherweise auch unterschiedlichen Induktionen in den Zähnen weitere modulationsbedingte Sättigungswellen, die jedoch zunächst nicht genauer betrachtet werden sollen.

Um eine tiefergehende Aussage über die genaue Ausprägung und Verteilung der Zahnsättigung treffen zu können, bedarf es zunächst einer tiefergehenden Betrachtung des Ständerfelds, welche im Folgenden durchgeführt wird.

Jede einzelne Spulenseite der Ständerwicklung steuert einen Beitrag zur Luftspaltinduktion im Bereich der Ständerzähne bei, welcher proportional zum Strom in der jeweiligen 


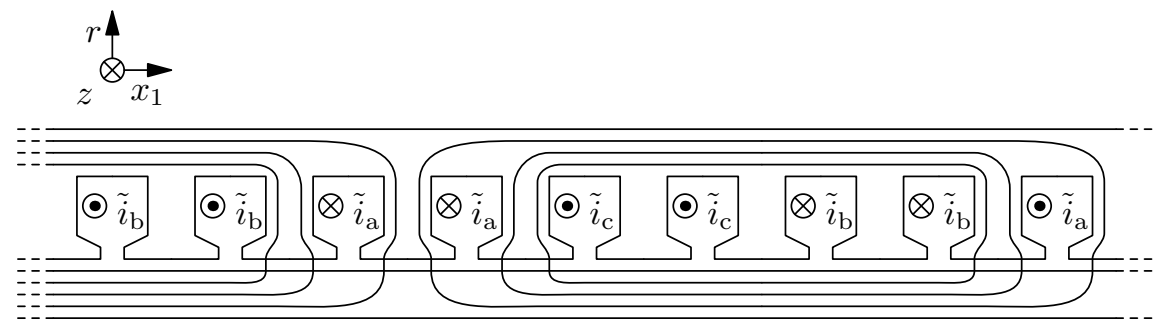

Bild 6.1: Schematische Darstellung des Felds aufgrund der Ströme in den Strängen „b“ und „,"; ausschließliche Darstellung der Feldlinien, welche zu einem Feld im Bereich der Zähne im Bereich von Strang ,a“ führen; es gilt $\tilde{i}=w_{\mathrm{N}} \cdot i$

Spule ist und sich mit Hilfe des Durchflutungssatzes (3.76) bestimmen lässt.

Um direkt die Induktion der gesamten Wicklung, also der Überlagerung aller Spulen zu erhalten, wird zunächst analog zu (3.23) im Bereich aller Zähne $(n)$ die in Form von komplexen Zeigern dargestellte zeitabhängige Felderregung

$$
\underline{V}_{\mathrm{z}(n)}= \begin{cases}\underline{c} & \text { für } n=0 \\ \underline{V}_{\mathrm{z}(n-1)}+\underline{\tilde{i}}_{(\mathrm{n})} & \text { sonst }\end{cases}
$$

bestimmt, woraus sich bei konstantem Luftspalt sowie $\mu_{\mathrm{Fe}} \rightarrow \infty$ die dazu proportionalen Induktionen $\underline{B}_{\mathrm{z}(n)}=\frac{\mu_{0}}{\delta} \underline{V}_{\mathrm{z}(n)}$ ergeben. Die Integrationskonstante $\underline{c}$ lässt sich dabei aus der Bedingung

$$
\sum_{n=1}^{N_{1}} \underline{\Phi}_{z(n)}=0
$$

bestimmen, welche bei einheitlichen Zahnbreiten,

$$
\sum_{n=1}^{N_{1}} \underline{B}_{\mathrm{z}(n)}=0 \quad \text { also hier auch } \quad \sum_{n=1}^{N_{1}} \underline{V}_{\mathrm{z}(n)}=0
$$

entspricht.

Bei $m_{1}=3$ ergibt sich herrührend von den Strängen „, b“ und „,c mit den Strömen gemäß (3.9) im Bereich der Zähne des Strangs ,a“ durch Überlagerung die in Bild 6.1 skizzierte Luftspaltinduktion

$$
B_{\mathrm{b}}(t)+B_{\mathrm{c}}(t)=-\sqrt{3} \tilde{B} \sin \left(\omega_{1} t+\varphi\right) .
$$

Dabei ist $\tilde{B}$ die Amplitude der Induktion, die von den Spulen eines Strangs im Bereich der Zähne, welche an Leiter der anderen Stränge grenzen, hervorgerufen wird.

Zusätzlich existiert ein Anteil der Induktion, der vom Strom im Strang ,a“ abhängig ist und sich auf die Zähne unterschiedlich auswirkt. Er lässt sich für den Zahn $n$ zu

$$
B_{\mathrm{a}(n)}(t)=\frac{k_{\mathrm{z}(n)}}{q_{1}} \tilde{B} \cos \left(\omega_{1} t+\varphi\right)
$$

bestimmen. Dabei ergibt sich $k_{\mathrm{z}(n)}$, wie in Bild 6.2a gezeigt, bei Einschichtwicklungen zur Anzahl der bei kleinerem Winkel $x_{1}$ liegenden benachbarten Spulenseiten des Strangs „a" abzüglich der bei größerem Winkel $x_{1}$ liegenden Spulenseiten. Wie in Bild 6.2b gezeigt, ist $k_{\mathrm{z}(n)}$ bei Zweischichtwicklungen gerade die Hälfte dieser Differenz. 


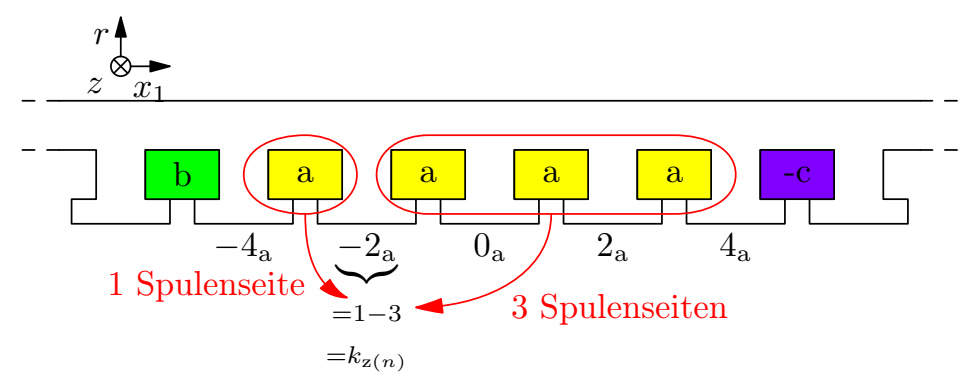

(a) Bestimmung bei Einschichtwicklungen

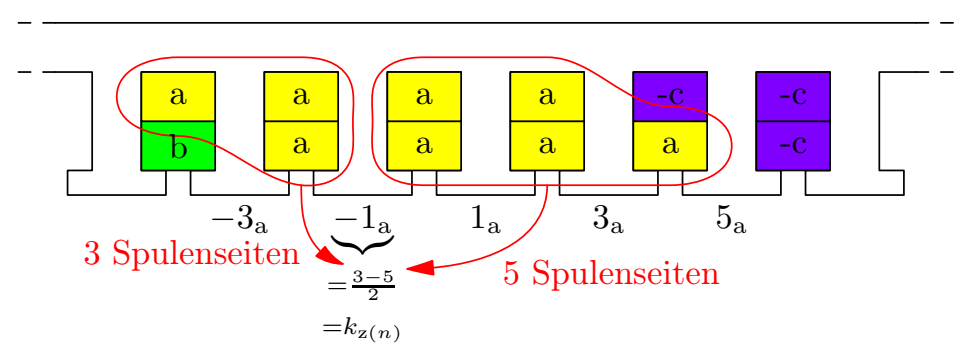

(b) Bestimmung bei Zweischichtwicklungen

Bild 6.2: Bestimmung von $k_{\mathrm{z}(n)}$ für den Strang ,a“

Tabelle 6.1: Bezogene Luftspaltinduktion $B_{\mathrm{kz}(n)}^{\prime}=B_{\mathrm{kz}(n)} / \tilde{B}$ im Bereich der Zähne bei verschiedenen Einschichtwicklungen

\begin{tabular}{l|ccccccc}
$q_{1} \notin 2 \mathbb{N}$ & $B_{-5}^{\prime}$ & $B_{-3}^{\prime}$ & $B_{-1}^{\prime}$ & - & $B_{+1}^{\prime}$ & $B_{+3}^{\prime}$ & $B_{+5}^{\prime}$ \\
$q_{1} \in 2 \mathbb{N}$ & $B_{-6}^{\prime}$ & $B_{-4}^{\prime}$ & $B_{-2}^{\prime}$ & $B_{0}^{\prime}$ & $B_{+2}^{\prime}$ & $B_{+4}^{\prime}$ & $B_{+6}^{\prime}$ \\
\hline$q=1$ & & & 2,00 & - & 2,00 & & \\
$q=2$ & & & 2,00 & 1,73 & 2,00 & & \\
$q=3$ & & 2,00 & 1,76 & - & 1,76 & 2,00 & \\
$q=4$ & & 2,00 & 1,80 & 1,73 & 1,80 & 2,00 & \\
$q=5$ & 2,00 & 1,83 & 1,74 & - & 1,74 & 1,83 & 2,00 \\
$q=6$ & 2,00 & 1,86 & 1,76 & 1,73 & 1,76 & 1,86 & 2,00
\end{tabular}

Eine Überlagerung der Anteile gemäß (6.5) und (6.6) führt letztendlich zu den resultierenden Luftspaltinduktionen im Bereich der an Leiter des Strangs „a" grenzenden Zähne, nämlich

$$
B_{\mathrm{kz}(n)}(t)=\tilde{B} \cdot \sqrt{3+\frac{k_{\mathrm{z}(n)}^{2}}{q_{1}^{2}}} \cos \left(\omega_{1} t+\varphi-\arctan \left(\frac{k_{\mathrm{z}(n)}}{\sqrt{3} q_{1}}\right)\right) .
$$

Zur Veranschaulichung von (6.7) sind beispielhaft in Tabelle 6.1 die Amplituden sowie in Tabelle 6.2 die zugehörigen Phasenverschiebungen der Luftspaltinduktionen im Bereich der verschiedenen Zähne für einige gängige Wicklungen dargestellt. Des Weiteren ist in Bild 6.3 ein beispielhafter Induktionsverlauf für zwei verschiedene Zeitpunkte dargestellt.

Es ist zu erkennen, dass sich bei gleichmäßiger Ständernutung und gleichmäßigem Wicklungsaufbau unter der Annahme unendlich hoher magnetischer Permeabilität des Eisens bei nicht vorhandener läuferseitig erregter Induktion unterschiedlich große Induktionsamplituden im Luftspalt zwischen Läufer und den einzelnen Zähnen ausbilden. Folglich ergeben sich bei gleichen Zahnquerschnitten auch unterschiedliche Induktionsamplituden 


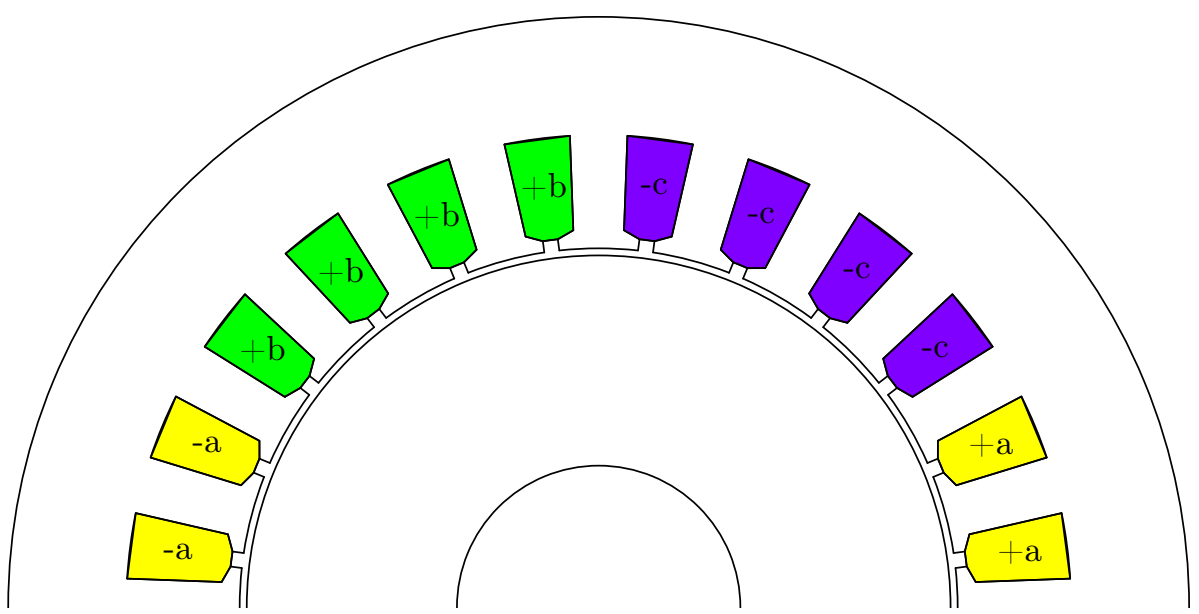

(a) Geometrie einer Ständerhälfte mit einem stark vereinfachten Läufer und gleichen Luftspaltweiten im Bereich der Zähne; $p=1 ; q_{1}=4$

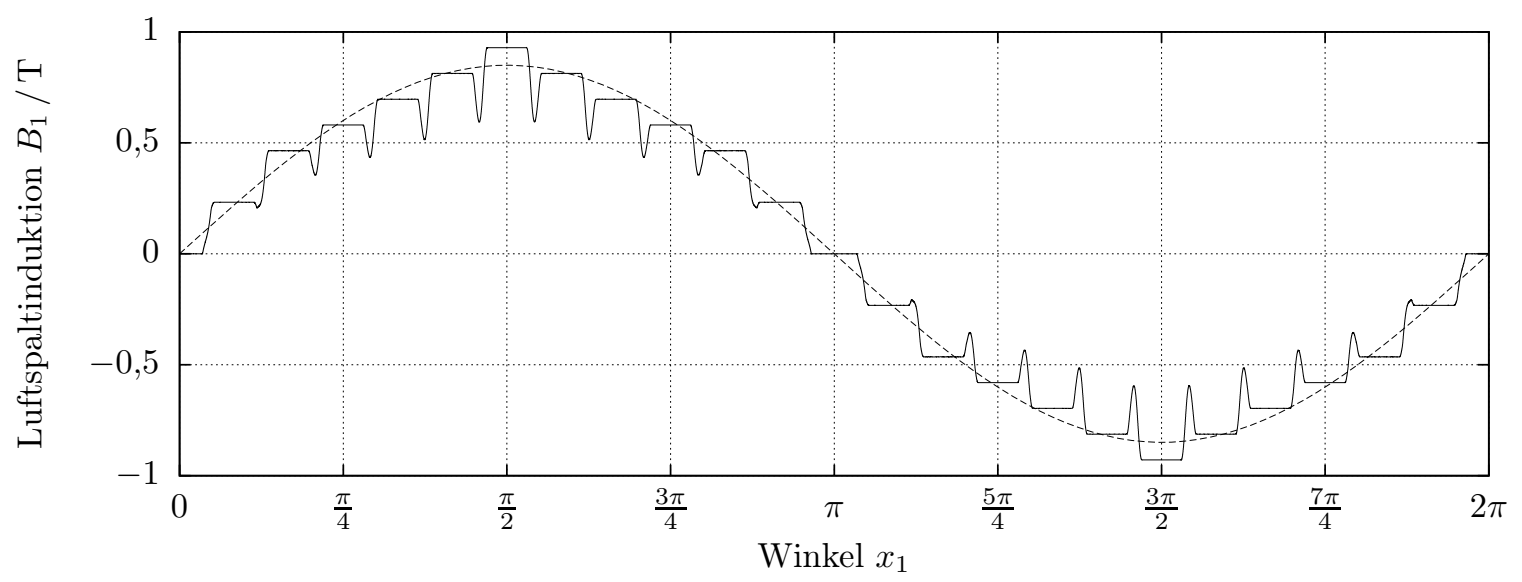

(b) Induktionsverlauf $B_{1}\left(x_{1}, t\right)$ für $\omega_{1} t=0$

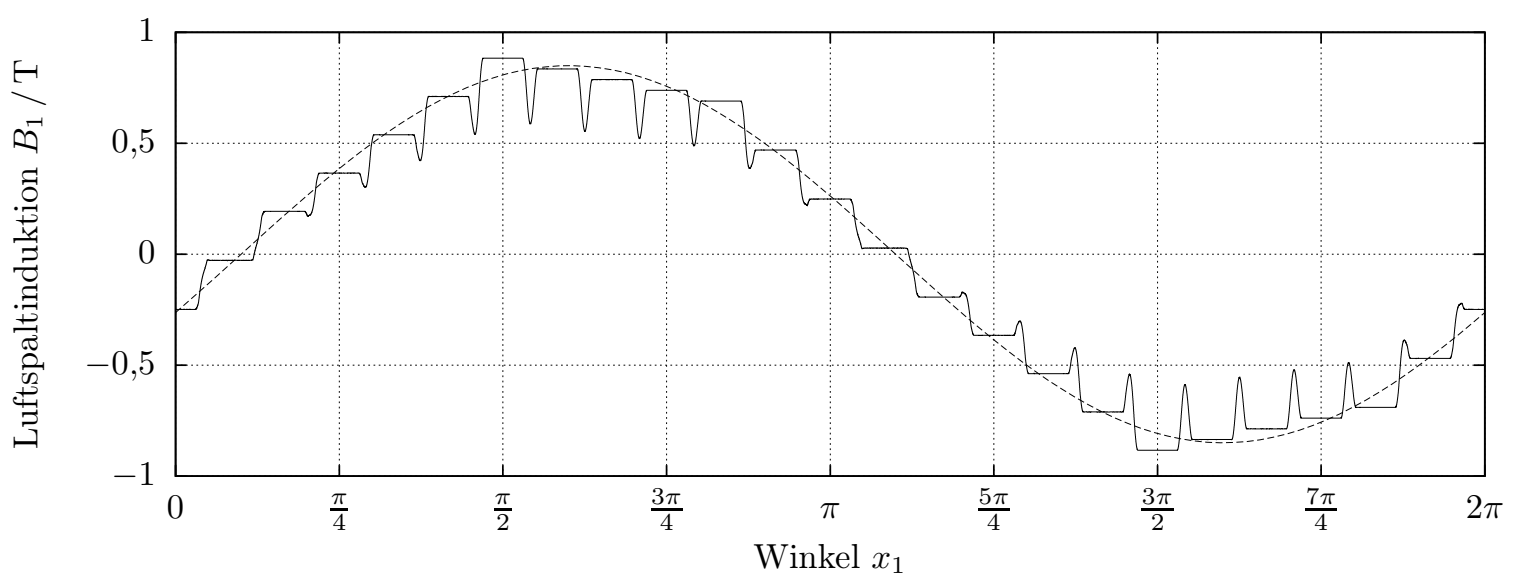

(c) Induktionsverlauf $B_{1}\left(x_{1}, t\right)$ für $\omega_{1} t=\frac{\pi}{10}$

Bild 6.3: Ständergeometrie und Induktionsverlauf bei gleicher Luftspaltweite im Bereich aller Ständerzähne; neben dem Induktionsverlauf ist die um den Kehrwert des Carter'schen Faktors $1 / k_{\mathrm{c}}$ vergrößerte Grundwelle der Induktion dargestellt. 
Tabelle 6.2: Phasenverschiebung $\varphi_{\mathrm{kz}(n)}$ der Luftspaltinduktion im Bereich der Zähne bei verschiedenen Einschichtwicklungen.

\begin{tabular}{|c|c|c|c|c|c|c|c|}
\hline $\begin{array}{l}q_{1} \notin 2 \mathbb{N} \\
q_{1} \in 2 \mathbb{N}\end{array}$ & $\begin{array}{l}\tilde{\varphi}_{-5} \\
\tilde{\varphi}_{-6}\end{array}$ & $\begin{array}{l}\tilde{\varphi}_{-3} \\
\tilde{\varphi}_{-4}\end{array}$ & $\begin{array}{l}\tilde{\varphi}_{-1} \\
\tilde{\varphi}_{-2}\end{array}$ & $\begin{array}{l}- \\
\tilde{\varphi}_{0} \\
\end{array}$ & $\begin{array}{l}\tilde{\varphi}_{+1} \\
\tilde{\varphi}_{+2} \\
\end{array}$ & $\begin{array}{l}\tilde{\varphi}_{+3} \\
\tilde{\varphi}_{+4} \\
\end{array}$ & $\begin{array}{l}\tilde{\varphi}_{+5} \\
\tilde{\varphi}_{+6}\end{array}$ \\
\hline$q=1$ & & & $0,00^{\circ}$ & - & $0,00^{\circ}$ & & \\
\hline$q=2$ & & & $0,00^{\circ}$ & $0,00^{\circ}$ & $0,00^{\circ}$ & & \\
\hline$q=3$ & & $0,00^{\circ}$ & $-0,89^{\circ}$ & - & $0,89^{\circ}$ & $0,00^{\circ}$ & \\
\hline$q=4$ & & $0,00^{\circ}$ & $-1,10^{\circ}$ & $0,00^{\circ}$ & $1,10^{\circ}$ & $0,00^{\circ}$ & \\
\hline$q=5$ & $0,00^{\circ}$ & $-1,11^{\circ}$ & $-0,59^{\circ}$ & - & $0,59^{\circ}$ & $1,11^{\circ}$ & $0,00^{\circ}$ \\
\hline$q=6$ & $0,00^{\circ}$ & $-1,05^{\circ}$ & $-0,89^{\circ}$ & $0,00^{\circ}$ & $0,89^{\circ}$ & $1,05^{\circ}$ & $0,00^{\circ}$ \\
\hline
\end{tabular}

in den Zähnen. In der Realität bedeutet dies, dass sich in den einzelnen Zähnen unterschiedlich hohe Sättigungen und Verluste ergeben.

Die so entstehenden Sättigungserscheinungen führen zu reduzierten magnetischen Leitwerten einzelner Zähne, welche sich für den Fall, dass Strang ,a“ gerade zu einer Magnetisierung in Richtung $0^{\circ}$ führt, analog zu $\lambda_{\text {sat2p }}\left(x_{1}, t\right)$ gemäß (6.1) durch Leitwertwellen der Form

$$
\lambda_{\text {sat } 6 k p}\left(x_{1}, t\right)=\sum_{k=1}^{\infty} \hat{\lambda}_{\text {sat } k} \cos \left(6 k p x_{1}+\pi\right)
$$

darstellen lassen.

Als dominierend ist hierin die Leitwertwelle der räumlichen Ordnungszahl $\nu=6 p \mathrm{zu}$ erwarten, welche in Kombination mit der ebenfalls dominierenden Felderregerwelle der räumlichen Ordnungszahl $p$ zu den Luftspaltinduktionswellen

$$
B_{1}\left(x_{1}, t\right)=\underbrace{\frac{\hat{\lambda}_{\mathrm{sat}} \cdot V_{1 \mathrm{p}}}{2}}_{=: B_{\mathrm{sat}}}\left[\begin{array}{c}
\cos \left(7 p x_{1}-\omega_{1} t+\varphi+\pi\right)+\ldots \\
\ldots+\cos \left(-5 p x_{1}-\omega_{1} t+\varphi-\pi\right)
\end{array}\right]
$$

führt.

Es treten also gemäß (6.9) Luftspaltinduktionswellen auf, die genau dieselben räumlichen Ordnungszahlen und Kreisfrequenzen wie die in Abschnitt 3.3 eingeführten Felderregerwellen der Ständerwicklung besitzen, gegenüber diesen jedoch eine Phasenverschiebung um den Winkel $\pi$ aufweisen. In Verbindung mit dem Gleichanteil der Leitwertwellen bedeutet dies, dass je zwei Induktionswellen entstehen, welche sich bei Amplitudengleichheit aufgrund ihrer gegensätzlichen Vorzeichen gerade auslöschen.

In der Realität bedeutet dies, dass die Induktionswellen, welche von den Oberwellen der ständerseitigen Felderregung herrühren, in einigen Betriebspunkten durch die hier aufgezeigten sättigungsbedingten Induktionswellen zumindest teilweise kompensiert werden. In anderen Betriebspunkten mit $B_{\text {sat }}>2 \cdot \hat{B}_{\nu}$ kann ihre Amplitude bei umgekehrtem Vorzeichen hingegen größer sein. 


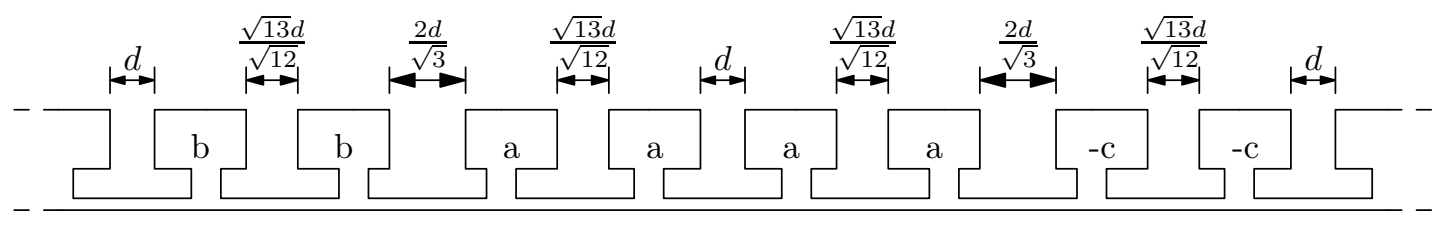

Bild 6.4: Übertriebene Darstellung der Anpassung der Zahnbreiten

\subsection{Variation der Zahnbreiten}

Wie im vorhergehenden Abschnitt gezeigt, ergeben sich mit den dort getroffenen Einschränkungen in einigen Ständerzähnen höhere Induktionsamplituden als in anderen. Die Ummagnetisierungsverluste, welche gemäß der zum Beispiel in [19] gezeigten Ausführungen gerade proportional zum Quadrat der Induktionsamplituden sind, unterscheiden sich folglich ebenfalls zwischen den einzelnen Zähnen.

Beispielhaft soll die Amplitude der Induktion $B_{\mathrm{z} 2}$ in den Zähnen „-2a “, beziehungsweise „2, “", bei einer Maschine mit $q_{1}=2$ Nuten je Pol und Strang betrachtet werden. Diese Induktion ist in etwa um den Faktor $2 / \sqrt{3}$ größer als die Amplitude der Induktion $B_{\mathrm{z} 0}$ in den Zähnen „, $0_{\mathrm{a}}{ }^{“}$. Es ergibt sich damit für die Zähne „, $0_{\mathrm{a}}{ }$ und, $2_{\mathrm{a}}{ }$, welche in der $r$ - $x_{1}$ Ebene die zunächst gleichen Flächen $A_{\mathrm{z} 0}$ und $A_{\mathrm{z} 2}$ einnehmen, die Beziehung zwischen den Ummagnetisierungsverlusten $P_{\mathrm{Fe} 0}$ und $P_{\mathrm{Fe} 2} \mathrm{zu}$

$$
B_{\mathrm{z} 0}^{2} \cdot A_{\mathrm{z} 0} \sim P_{\mathrm{Fe} 0} \approx\left(\frac{\sqrt{3}}{2}\right)^{2} P_{\mathrm{Fe} 2} \sim\left(\frac{\sqrt{3}}{2}\right)^{2} B_{\mathrm{z} 2}^{2} \cdot A_{\mathrm{z} 2} .
$$

Werden die Zähne hingegen nicht flächengleich mit $A_{\mathrm{z} 0}=A_{\mathrm{z} 2}$ ausgeführt, sondern ihre Breiten, nicht aber die Nutpositionen angepasst, wie in Bild 6.4 dargestellt, so ergeben sich die Zahnflächen $\breve{A}_{\mathrm{z} 0}$ und $\breve{A}_{\mathrm{z} 2}$ und die bei ausschließlich ständerseitiger Felderregung gleichen Zahninduktionen $\breve{B}_{\mathrm{z} 0}=\breve{B}_{\mathrm{z} 2}$.

Die Ummagnetisierungsverluste in den Ständerzähnen werden damit zu

$$
\breve{P}_{\mathrm{Fe} 0}=P_{\mathrm{Fe} 0}\left(\frac{1}{2}+\frac{1}{\sqrt{3}}\right)^{(2-1)} \quad \text { und } \quad \breve{P}_{\mathrm{Fe} 2}=P_{\mathrm{Fe} 2}\left(\frac{\sqrt{3}}{2}\left(\frac{1}{2}+\frac{1}{\sqrt{3}}\right)\right)^{(2-1)}
$$

sodass die Summe der Verluste $\breve{P}_{\mathrm{Fe}}=\breve{P}_{\mathrm{Fe} 0}+\breve{P}_{\mathrm{Fe} 2 \mathrm{zu}}$

$$
\breve{P}_{\mathrm{Fe}}=\left[\frac{3}{7}\left(\frac{1}{2}+\frac{1}{\sqrt{3}}\right)+\frac{4}{7}\left(\frac{\sqrt{3}}{2}\left(\frac{1}{2}+\frac{1}{\sqrt{3}}\right)\right)\right] \cdot P_{\mathrm{Fe}}
$$

wird.

Es gilt folglich $\breve{P}_{\mathrm{Fe}}=0,995 P_{\mathrm{Fe}}$, womit sich die Ummagnetisierungsverluste in den Ständerzähnen bei der vorgeschlagenen Anpassung der Zahnbreiten um lediglich 0,5\% reduzieren würden.

Gleichzeitig würde es aufgrund der relativ zum Ständerstrom abgesenkten Induktionen in den Zähnen, welche gegenüber einer gleichmäßigen Geometrie verbreitert wurden, erst 


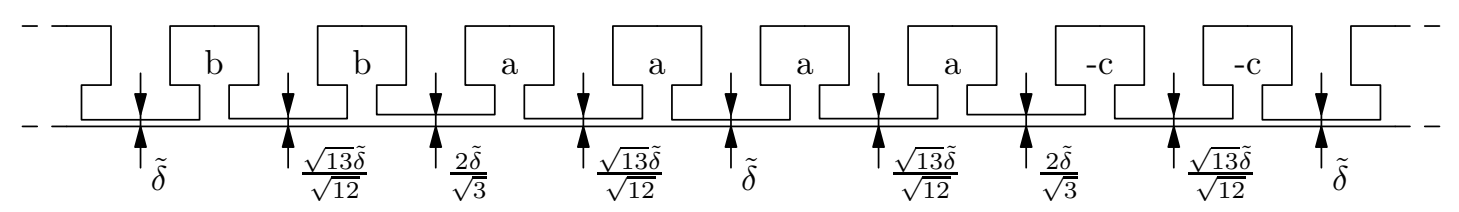

Bild 6.5: Übertriebene Darstellung der Anpassung der Luftspaltweite

bei größeren Ständerströmen zu signifikanten Sättigungserscheinungen kommen, beziehungsweise es käme bei gleicher Ständerstromamplitude und angepassten Zahnbreiten gegenüber der gleichmäßig genuteten Geometrie eventuell zu geringeren Zahnsättigungen. Folglich führt die gezeigte Anpassung der Zahnbreiten im Bereich hoher Ständerströme zu einem größeren Verhältnis von Luftspaltinduktionsamplitude zu Ständerstromamplitude und somit zu geringeren Stromwärmeverlusten in der Ständerwicklung.

Zusätzlich zur Auswirkung auf die Verluste, führt die vorgeschlagene Anpassung der Zahnbreiten bei ausschließslicher Ständerfelderregung aufgrund der in allen Zähnen gleichen Induktionen dazu, dass die im vorhergehenden Absatz aufgezeigten Leitwertwellen gemäßs (6.8) nicht auftreten.

\subsection{Variation der Luftspaltweite}

In Abschnitt 6.1 wurde gezeigt, dass die Zahnsättigung, welche eine Erhöhung des magnetischen Widerstands in einzelnen Ständerzähnen mit sich bringt, zur Auslöschung einiger Ständerinduktionswellen führen kann.

Es drängt sich damit auf, anstelle der im vorhergehenden Abschnitt vorgeschlagenen Zahnbreitenvariation eine Zahnhöhen- und damit Luftspaltweitenvariation einzuführen. Hierzu müssten die Luftspaltweiten zu

$$
\delta_{\mathrm{kz}(n)}^{\prime}=\tilde{\delta} \cdot \sqrt{3+\frac{k_{\mathrm{z}(n)}^{2}}{q_{1}^{2}}},
$$

also proportional zu $B_{\mathrm{kz}(n)}(t)$ gemäß (6.7) gewählt werden, damit sich bei beliebigem $\tilde{\delta}$ die in Bild 6.5 dargestellte Variation zur Unterdrückung der Induktionsoberwellen ergibt. Es entsteht somit die beispielhaft in Bild 6.6] dargestellte Luftspaltinduktion.

Mit der Modulation entsprechend (6.13) entstehen dabei den Sättigungswellen gemäß (6.8) ähnliche zusätzliche Leitwertwellen der Form

$$
\lambda\left(x_{1}\right)=\hat{\lambda}_{\nu} \cos \left(\nu x_{1}+\pi\right) \quad \text { mit } \quad \nu=n 6 p ; n \in \mathbb{N}
$$

Beispielhaft sind die entstehenden Amplituden $\hat{\lambda}_{\nu}$ für einige Wicklungen in Tabelle 6.3 aufgeführt. Die Berechnung der Werte basiert hierbei auf der Voraussetzung regelmäßig angeordneter vernachlässigbar schmaler Nuten, womit sich eine Treppenfunktion $\delta\left(x_{1}\right)$ ergibt, die sich leicht in eine Fourierreihe überführen lässt.

Es ist naheliegend, die Luftspaltweite $\tilde{\delta}$ in (6.13) derart zu wählen, dass sich mit der Luftspaltweitenmodulation derselbe Gleichanteil der Luftspaltleitwertverteilung ergibt, wie 


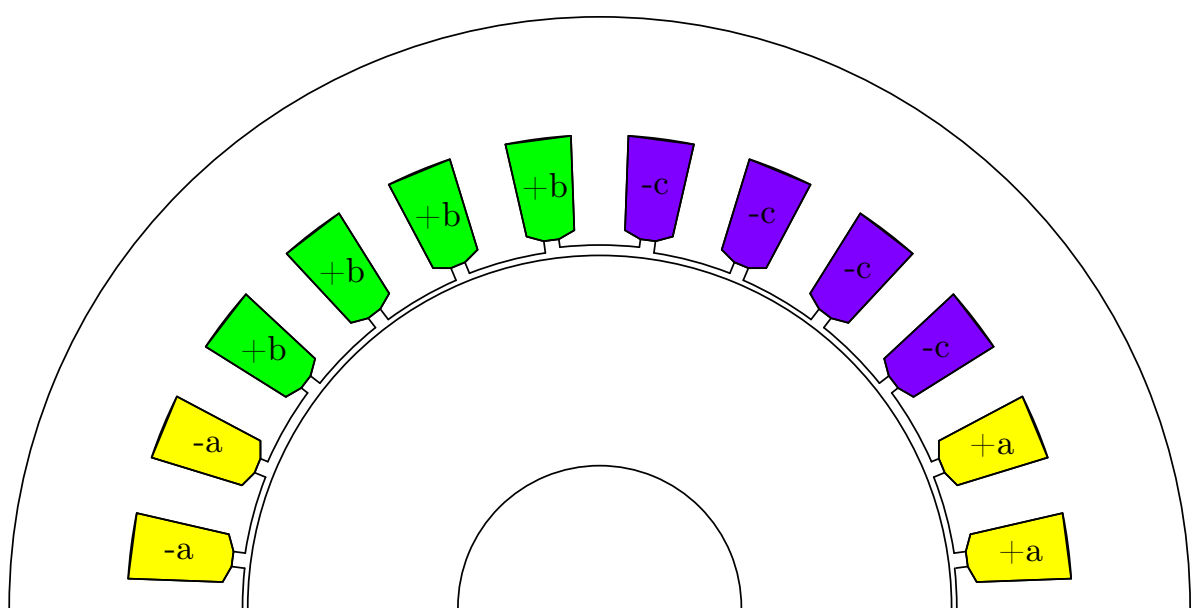

(a) Geometrie einer Ständerhälfte mit einem stark vereinfachten Läufer und fünffach überhöhter Anpassung der Luftspaltweiten im Bereich der Zähne; $p=1 ; q_{1}=4$

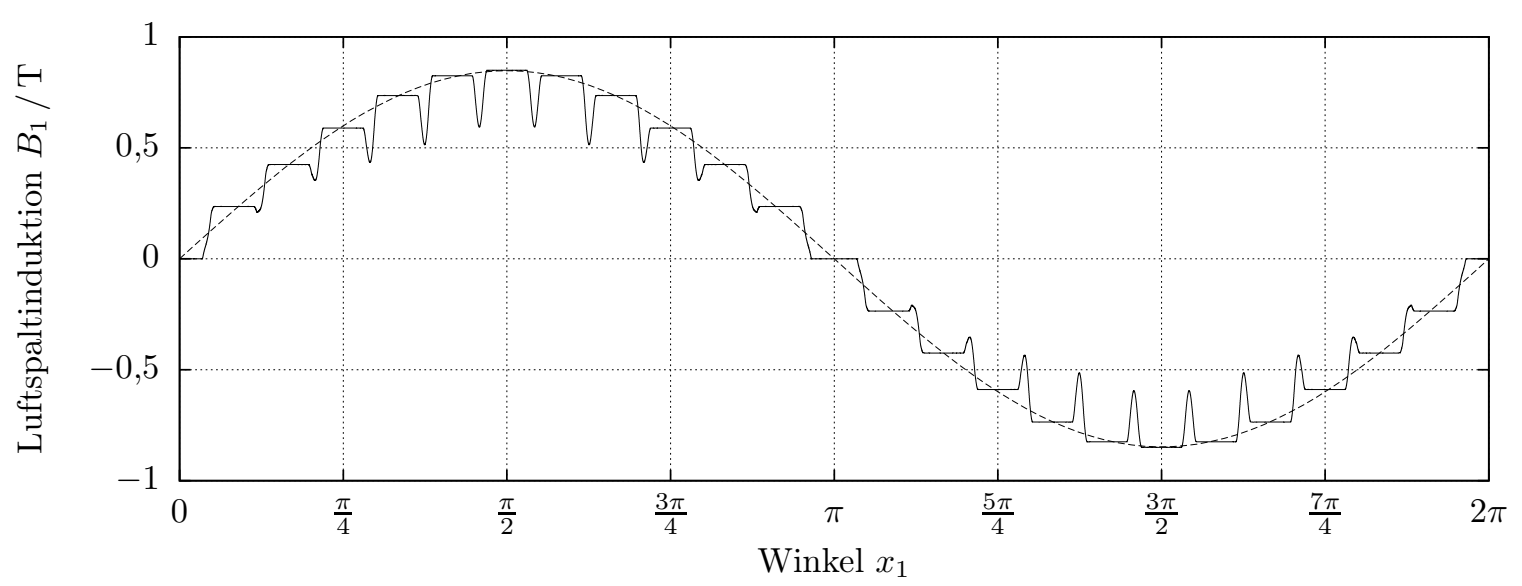

(b) Induktionsverlauf $B_{1}\left(x_{1}, t\right)$ für $\omega_{1} t=0$

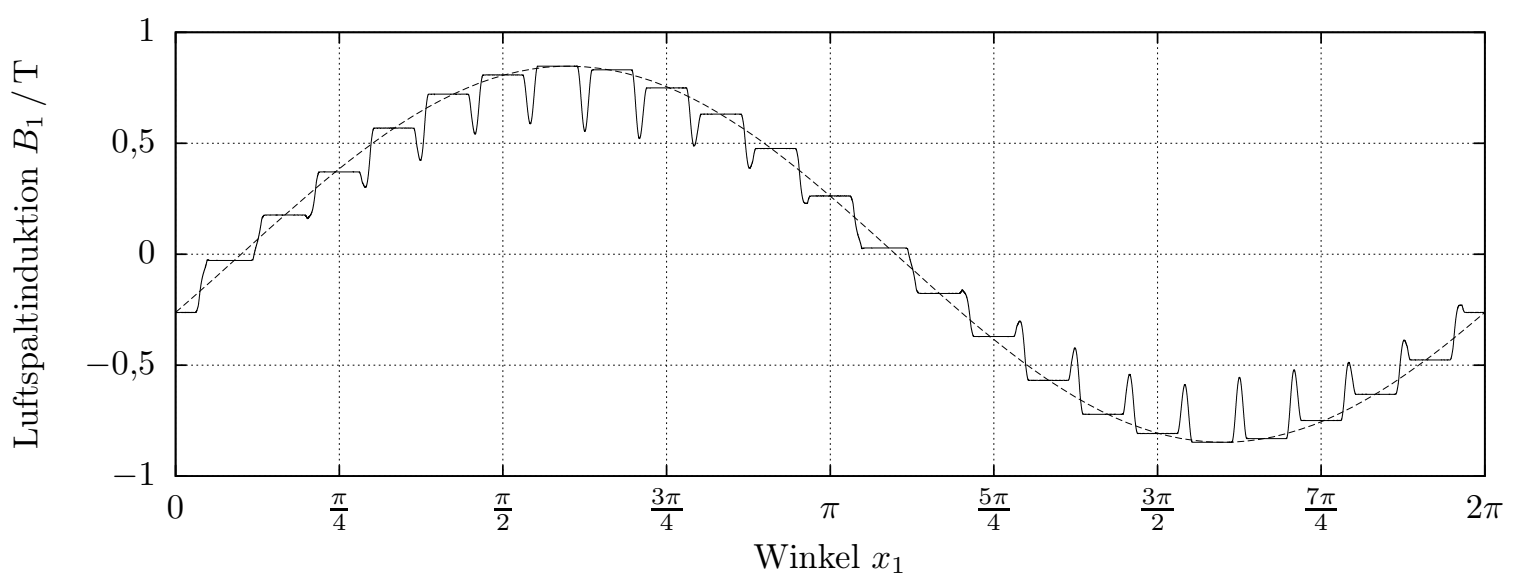

(c) Induktionsverlauf $B_{1}\left(x_{1}, t\right)$ für $\omega_{1} t=\frac{\pi}{10}$

Bild 6.6: Ständergeometrie und Induktionsverlauf bei über dem Umfang unterschiedlichen Luftspaltweiten im Bereich der Ständerzähne; neben dem Induktionsverlauf ist die um den Kehrwert des Carter'schen Faktors $1 / k_{\mathrm{c}}$ vergrößerte Grundwelle der Induktion dargestellt. 
Tabelle 6.3: Amplituden $\hat{\lambda}_{\nu}^{\prime}$ der Luftspaltleitwertwellen aufgrund der Luftspaltweitenmodulation bei Einschichtwicklung bezogen auf den mittleren Luftspaltleitwert $\lambda_{0}$ mit $\hat{\lambda}_{\nu}=\lambda_{0} \hat{\lambda}_{\nu}^{\prime}$

\begin{tabular}{rrrrrrr}
\hline$q_{1}$ & $\nu=6 p$ & $\nu=12 p$ & $\nu=18 p$ & $\nu=24 p$ & $\nu=30 p$ & $\nu=36 p$ \\
\hline 1 & 0,000 & 0,000 & 0,000 & 0,000 & 0,000 & 0,000 \\
2 & 0,091 & 0,000 & $-0,030$ & 0,000 & 0,018 & 0,000 \\
3 & 0,068 & $-0,034$ & 0,000 & 0,017 & $-0,014$ & 0,000 \\
4 & 0,064 & $-0,019$ & 0,021 & 0,000 & $-0,013$ & 0,006 \\
5 & 0,062 & $-0,016$ & 0,010 & $-0,016$ & 0,000 & 0,010 \\
6 & 0,061 & $-0,014$ & 0,008 & $-0,007$ & 0,012 & 0,000 \\
$\infty$ & 0,060 & $-0,012$ & 0,005 & $-0,003$ & 0,002 & $-0,001$ \\
\hline
\end{tabular}

ohne die Modulation. Dies würde jedoch bedeuten, dass der in der Regel bei Induktionsmaschinen ohnehin schon enge Luftspalt im Bereich der längeren Zähne weiter verringert würde. Damit ergäben sich zum Beispiel für die magnetischen Kräfte, die durch Exzentrizitäten auftreten und proportional vom Quadrat der umgekehrt proportional zur Luftspaltweite stehenden Induktion abhängen, trotz gleicher mittlerer Luftspaltweite größere Werte. Es muss folglich von Fall zu Fall genau geprüft werden, in welchem Rahmen $\tilde{\delta}$ gewählt werden kann.

Die hier vorgestellte Variation der Luftspaltweite basiert auf einem Modell mit ausschließlicher ständerseitiger Felderregung. Kommt es jedoch auch, wie bei Induktionsmaschinen zu erwarten, zu einer läuferseitigen Felderregung, so führen die zusätzlichen Luftspaltleitwertwellen, welche sich aufgrund der Variation der Luftspaltweite ergeben, zu genau den Induktionswellen, welche durch die Variation ausgelöscht werden sollten.

Folglich scheint die Variation der Luftspaltweite vornehmlich für Maschinen mit einer geringen läuferseitigen Felderregung geeignet zu sein, also zum Beispiel für Induktionsmaschinen mit einer hohen Überlastbarkeit, aber auch zum Beispiel für Synchronreluktanzmaschinen.

\subsection{Phasenwinkelkorrektur}

In Tabelle 6.2 wurde bereits gezeigt, dass sich für regelmäßig angeordnete Ständerzähne bei $q_{1}>2$ Phasenwinkel $\varphi_{\mathrm{z}(n)}=-\arg \left(\underline{V}_{\mathrm{z}(n)}\right)$ der Felderregung im Luftspalt an den Zähnen ergeben, welche nicht exakt mit der Lage der Zähne korrespondieren. Es gilt also nicht für alle $(n)=\left\{1,2,3, \ldots, N_{1}\right\}$ Zähne

$$
x_{\mathrm{z}(n)}=\frac{\varphi_{\mathrm{z}(n)}}{p}+k \cdot \frac{2 \pi}{p} ; k \in \mathbb{N}
$$

Um ein möglichst oberwellenarmes Luftspaltfeld zu erhalten, wäre es jedoch wünschenswert, dass (6.15) für alle $(n)$ erfüllt ist. Dies lässt sich erreichen, indem die Zahnpositionen entsprechend der bereits in der richtigen Reihenfolge vorliegenden Phasenverschiebungen der Zahninduktionen $\varphi_{\mathrm{z}(n)}$ durch Verschieben der Ständernuten angepasst werden. 
Die hierfür notwendigen Nutpositionen

$$
x_{1(n)}=2 x_{z(n)}+2 \tilde{\varphi}-x_{1(n+1)}
$$

ergeben sich dabei aus dem Gleichungssystem

$$
\frac{1}{2} \cdot\left[\begin{array}{ccccc}
1 & 1 & 0 & \ldots & 0 \\
0 & 1 & 1 & \ldots & 0 \\
0 & 0 & 1 & \ldots & 0 \\
\vdots & \vdots & \vdots & \ddots & \vdots \\
1 & 0 & 0 & \ldots & 1
\end{array}\right] \cdot\left[\begin{array}{c}
x_{1(1)} \\
x_{1(2)} \\
x_{1(3)} \\
\vdots \\
x_{1\left(N_{1}\right)}
\end{array}\right]=\left[\begin{array}{c}
x_{\mathrm{z}(1)}+\tilde{\varphi} \\
x_{\mathrm{z}(2)}+\tilde{\varphi} \\
x_{\mathrm{z}(3)}+\tilde{\varphi} \\
\vdots \\
x_{\mathrm{z}\left(N_{1}\right)}+\tilde{\varphi}-\pi
\end{array}\right]
$$

welches sich wiederum bei einer zunächst beliebigen Verdrehung des Ständers $\tilde{\varphi}$ aus den Zahnpositionen

$$
x_{\mathrm{z}(n)}=\frac{x_{1(n)}+x_{1(n+1)}}{2}
$$

und (6.15) ergibt.

Es verbleibt die Nutposition $x_{1\left(N_{1}\right)}=-x_{10}$ für gerade Ständernutzahlen, also $N_{1} \in 2 \mathbb{N}$, als Freiheitsgrad, wohingegen sich für ungerade Ständernutzahlen, also $N_{1} \in 2 \mathbb{N}+1$, die zusätzliche Bedingung

$$
x_{1\left(N_{1}\right)}=x_{\mathrm{z}\left(N_{1}\right)}+\tilde{\varphi}-\pi+\sum_{n=1}^{N_{1}-1}(-1)^{n} \cdot\left[x_{\mathrm{z}(n)}+\tilde{\varphi}\right]
$$

ergibt. Einschränkend sei dabei angemerkt, dass auch der Wertebereich des Freiheitsgrads für gerade Ständernutzahlen, also der von $x_{10}$, aufgrund der erforderlichen minimalen Zahnbreiten begrenzt ist.

Letztendlich ergibt sich bei Anwendung der Phasenwinkelkorrektur in Kombination mit der Variation der Luftspaltweite eine Luftspaltinduktion, wie sie beispielhaft in Bild 6.7 dargestellt ist. Bei genauem Hinsehen lässt sich dabei erkennen, dass die Induktionsgrundwelle in Bild 6.7 im Gegensatz zu der in Bild 6.6 genau mittig durch die Plateaus der Luftspaltinduktion verläuft.

\subsubsection{Einfluss der Phasenwinkelkorrektur auf die Variation der Luftspaltweite}

Die in Abschnitt 6.3 vorgeschlagene Variation der Luftspaltweite verfolgt das Ziel, dass sich die ständerseitig erregte Luftspaltinduktion über der Winkelkoordinate $x_{1}$ zu $N_{1}$ phasenverschobenen Stufen ergibt, von denen jede einzelne eine mit der Frequenz $f_{1}$ zeitlich harmonische Induktion derselben Amplitude aufweist.

Durch Anwendung der vorgeschlagenen Phasenwinkelkorrektur ergeben sich unterschiedlich breite Ständerzähne bei gleich breiten Ständernuten. Wie in Bild 6.7 zu erkennen ist, liegen die Plateaus der Zahninduktionen damit jeweils mittig auf der Induktionshauptwelle. Zur Ausbildung eines möglichst oberwellenarmen Induktionsspektrums müssten jedoch nicht die Plateaus, sondern näherungsweise die Mittelwerte der Induktionen zwischen je zwei benachbarten Nutmitten dieselben Amplituden aufweisen. Das bedeutet aufgrund der 


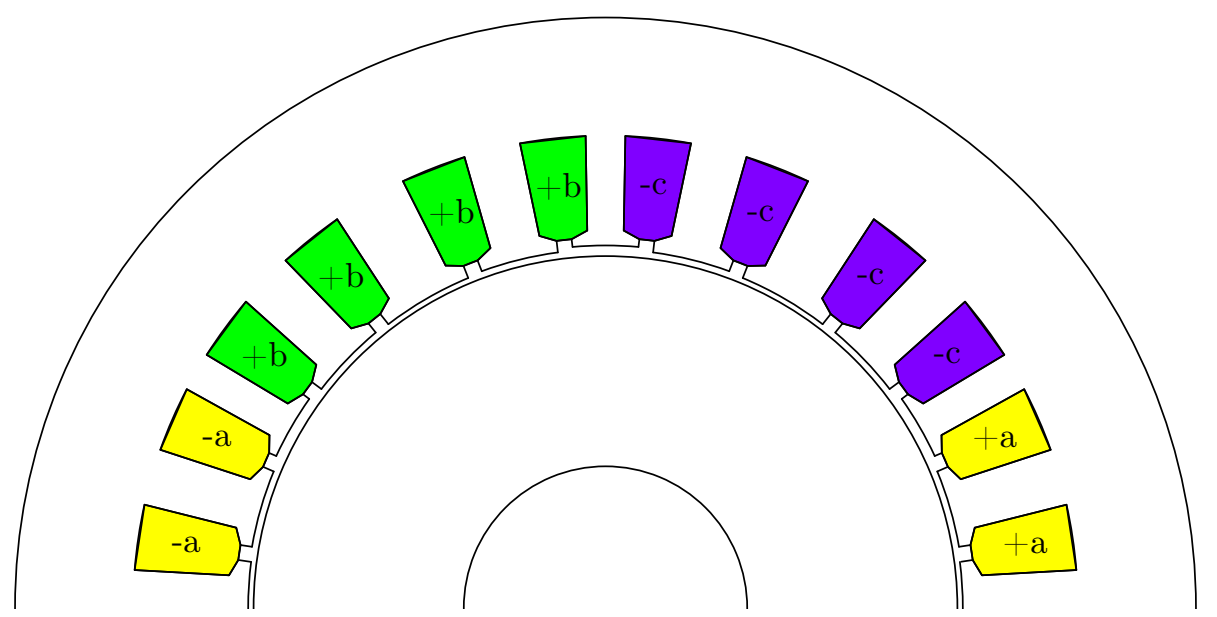

(a) Geometrie einer Ständerhälfte mit einem stark vereinfachten Läufer und fünffach überhöhter Anpassung der Luftspaltweiten im Bereich der Zähne; $p=1 ; q_{1}=4$

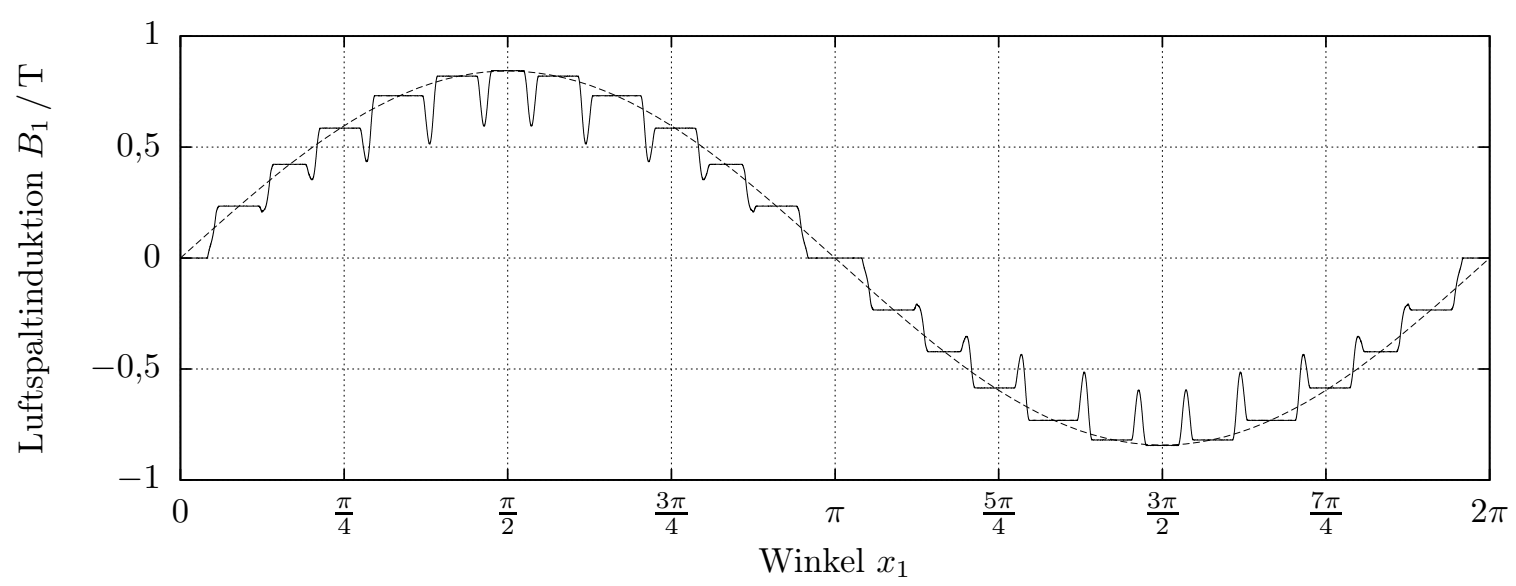

(b) Induktionsverlauf $B_{1}\left(x_{1}, t\right)$ für $\omega_{1} t=0$

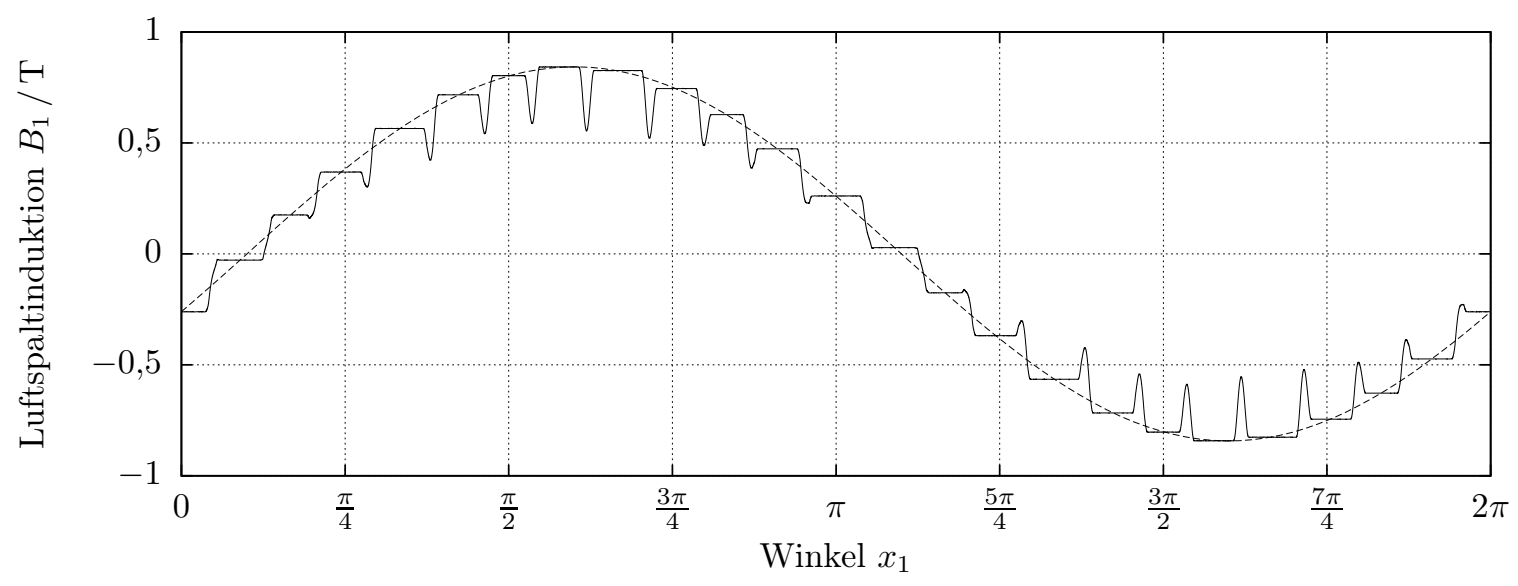

(c) Induktionsverlauf $B_{1}\left(x_{1}, t\right)$ für $\omega_{1} t=\frac{\pi}{10}$

Bild 6.7: Ständergeometrie und Induktionsverlauf bei über dem Umfang unterschiedlichen Luftspaltweiten im Bereich der Ständerzähne und Anpassung der Nutpositionen; neben dem Induktionsverlauf ist die um den Kehrwert des Carter'schen Faktors 1/ $k_{\mathrm{c}}$ vergrößerte Grundwelle der Induktion dargestellt. 
unterschiedlichen Verhältnisse von Zahn- zu Nutbreiten, dass eine nachgelagerte erneute Variation der Ständernutweiten zur Kompensation dieses Effekts durchgeführt werden muss. Ein Beispiel hierfür wird in Abschnitt 7.4.2.2 aufgezeigt.

Es sei angemerkt, dass der erläuterte Einfluss der unterschiedlichen Verhältnisse zwischen Zahn- und Nutbreiten durch eine Anpassung der Nutbreiten, wie dies in Abschnitt [7.3.1] für die Läufernuten vorgeschlagen wird, kompensiert werden könnte. In aller Konsequenz ergäben sich aufgrund der damit verbundenen verschiedenen Nutflächen jedoch unterschiedliche Spulenwindungszahlen im Ständer.

\subsubsection{Nachteile der Phasenwinkelkorrektur}

Ein Nachteil der Phasenwinkelkorrektur ist das eventuelle Auftreten weiterer Schwingungsformen des Ständerjochs, welche sich aus den Radialkräften an den Zähnen ergeben.

Wie in Abschnitt 4.4 beschrieben, kommt es durch die Übertragung der am Luftspalt wirkenden Radialkräfte über die Zähne auf das Joch bei gleichmäßiger Anordnung der Ständerzähne gemäß (4.57) zu einer Modulation mit der Ständernutzahl $N_{1}$. Werden die Ständernuten hingegen gemäß der vorgeschlagenen Phasenwinkelkorrektur ungleichmäßig angeordnet, so löst sich die in (4.57) vorausgesetzte strenge Periodizität der Ständerzahnpositionen und -größen auf.

Aufgrund der nun vorliegenden zonenweisen Periodizität der Zahnbreiten, welche sich aus der vorgeschlagenen Phasenwinkelkorrektur ergibt, entstehen aus einer Radialkraftwelle der räumlichen Ordnungszahl $\nu$ die zusätzlichen Jochzugwellen der Ordnungszahlen

$$
\mu=\nu+k \cdot 2 m_{1} p \text { mit } k=\{0, \pm 1, \pm 2, \ldots\} .
$$

Das Spektrum der räumlichen Ordnungszahlen der Jochzugwellen wird folglich voller. Hierbei ist zu beachten, dass sich die Ausprägung dieses Effekts für gerade Ständernutzahlen mit Hilfe des Freiheitsgrades $x_{1(1)}$ beeinflussen lässt. 


\section{Entwurf und Untersuchung von Beispielmaschinen}

Anhand ausgesuchter Beispiele soll in diesem Kapitel die Auswirkung einiger gezielt ungleichmäßiger Anordnungen und Geometrien von Ständer oder Läuferzähnen, also auch Ständer oder Läufernuten, auf die Maschineneigenschaften untersucht werden.

\subsection{Entwurfsgang}

Zum Entwurf der nachfolgenden Maschinen wurde das eigens für diesen Einsatz entwickelte analytische Rechenprogramm GRION genutzt, welches auf den in den vorangegangenen Kapiteln vorgestellten Berechnungsmethoden basiert. Hiermit ist eine schnelle Beurteilung und somit numerische Optimierung der Maschinengeometrien möglich. Durch eine automatisierte Kopplung mit dem Finite-Elemente-Programm FEMAG, das für diese Aufgabe eigens erweitert wurde, können dabei auch analytisch schwer erfassbare Effekte betrachtet werden.

Gemeinsam haben alle nachfolgend vorgestellten Geometrien, dass sie aus einem regelmäßig aufgebauten Blechschnitt sowohl für den Ständer als auch für den Läufer abgeleitet sind. Ausgehend hiervon bietet es sich an, eine Analyse der bezüglich der Geräuschentwicklung dominierenden Effekte durchzuführen. Bereits mit Papier und Bleistift lassen sich daraufhin Geometrien entwickeln, die zu einer Reduktion der Anteile an der Schallleistung führen, welche gemäß der vorherigen Analyse auf den dominierenden Wechselwirkungen in der Maschine beruhen. Aufgrund der insbesondere bei unregelmäßig aufgebauten Maschinen praktisch nicht mehr überschaubaren Zusammenhänge kann dabei jedoch nicht erwartet werden, dass manuell eine hinsichtlich aller signifikanten Wechselwirkungen optimale Geometrie gefunden wird. Vielmehr lassen sich durch diese manuellen Vorarbeiten dem Zufallsprinzip oft überlegene Startwerte für eine numerische Optimierung der Maschinengeometrien finden.

Die numerische Optimierung der Maschinengeometrien erfolgte für die nachfolgenden Beispiele ausschließlich mit Hilfe des eingangs erwähnten Berechnungsprogramms GRION. Dabei werden für einen oder mehrere Betriebspunkte unter anderem die Pendeldrehmomente, Schallleistungen und der Wirkungsgrad berechnet und bewertet. Die Schwierigkeit liegt hierbei in der Gestaltung der Bewertungsfunktion, wobei der Fokus bei der Optimierung der meisten der nachfolgenden Beispiele primär auf der Reduktion der Geräuschemission lag.

Die Bewertung der Schallleistung bezieht dabei zum einen die Leistungen einzelner Töne, aber auch zum Beispiel die Lästigkeit ihrer Kombinationen mit ein. Gleichzeitig muss die 
Tabelle 7.1: Technische Daten der Basismaschinen

\begin{tabular}{lcc|c|c|c}
\hline & & & \multicolumn{3}{|c}{ Maschine } \\
& & ${ }^{2} \mathrm{~A}^{\prime}$ & „B“ & „C“ \\
\hline Bemessungsleistung & $P_{\mathrm{N}}=$ & $3 \mathrm{~kW}$ & $3 \mathrm{~kW}$ & $15 \mathrm{~kW}$ \\
Polpaarzahl & $p=$ & 2 & 4 & 2 \\
Anzahl der Ständernuten & $N_{1}=$ & 24 & 36 & 48 \\
Anzahl der Läufernuten & $N_{2}=$ & 22 & 33 & 40 \\
Lochzahl & $q_{1}=$ & 2 & $\frac{3}{2}$ & 4 \\
Datenquelle & & & eigener Entwurf & real ausgeführt \\
\hline
\end{tabular}

Bewertungsfunktion die Leistungsdaten und nicht zuletzt die Fertigbarkeit der Maschine berücksichtigen. Letzteres definiert sich dabei zum Beispiel über minimale Zahnbreiten oder Nutquerschnitte, sodass sich mechanisch stabile Blechschnitte ergeben, in die in der Regel mit einem Druckgussverfahren ein Käfig eingebracht werden kann.

Angemerkt werden soll an dieser Stelle, dass die Rechengenauigkeit bei der genannten numerischen Optimierung von fundamentaler Bedeutung sein kann. Als Beispiel sei hierfür angenommen, dass eine Maschine ausschließlich für ihren Bemessungspunkt optimiert wird. Die niedrigste angeregte Eigenform der Ständerschwingung bei gleichmäßiger Nutanordnung sei $\nu \geq 4$. Durch eine Variation der Läufernutpositionen können nun zum Beispiel zusätzliche Jochzugwellen mit $\nu=2$ auftreten, also mit gegenüber $\nu \geq 4$ größerer relativer Schallleistung. Diese Jochzugwellen können außerdem eine bei gleichmäßiger Geometrie nicht angeregte Eigenfrequenz der zusätzlich angeregten Eigenform mit $\nu=2$ treffen und zum Beispiel auf einer Wechselwirkung zweier Induktionswellen mit relativ niedriger und relativ hoher Amplitude basieren. Für diesen Fall wird deutlich, dass bereits eine Induktionswelle mit verhältnismäßig niedriger Amplitude signifikant zum Geräusch beitragen kann, weshalb auch verhältnismäßig kleine Amplituden, welche einige Zehnerpotenzen unterhalb derer der größten Induktionswellen liegen, bei der Berechnung berücksichtigt werden müssen. Folglich ergibt sich mit dem aufgeweiteten Spektrum bei Variation der Ständer- und/oder Läufergeometrie und der damit verbundenen erhöhten Zahl von möglichen Kombinationen verschiedener Wellen ein signifikanter Rechenaufwand.

\subsection{Gleichmäßige Geometrien}

Die Untersuchungen in diesem Kapitel werden auf der Basis von drei konventionellen dreisträngigen Maschinen durchgeführt, deren wichtigste Daten in Tabelle 7.1 aufgelistet sind. Es handelt sich dabei um zwei reale ausgeführte Maschinen, von denen Messwerte vorliegen, sowie um eine selbst entworfene Maschine.

Die grafischen Darstellungen der Geometrien der realen Maschinen sind im Folgenden aus Gründen des Datenschutzes leicht verfälscht. 


\section{Maschine „A“}

Maschine „A“ ist eine selbst entworfene Maschine, anhand derer einige Besonderheiten der ungleichmäßsigen Geometrien aufgezeigt werden sollen.

\section{Maschine „B“}

Als Beispielmaschine „, $\mathrm{B}$ “ dient eine real ausgeführte achtpolige Maschine, deren Geräusch auffällig geworden ist. Sie besitzt $N_{2}=33$ Läufernuten und $N_{1}=36$ Ständernuten, womit sich $q_{1}=3 / 2$ Nuten je Pol und Strang ergeben. Der Blechschnitt der Maschine ist in Bild $7.3 \mathrm{a}$ dargestellt.

Für diese Maschine liegen Messwerte der Schallleistung für den Betrieb mit einem unmodulierten Läufer sowie für den später vorgestellten modulierten Läufer vor. Diese Messwerte sind in Bild 7.2 gezeigt.

\section{Maschine „C“}

Die Beispielmaschine „C“ zeigt im Betrieb keine akustischen Auffälligkeiten. Sie soll aufgrund ihrer im Vergleich zur Maschine „B“ einfachen Geometrie mit einer Ganzloch-Einschichtwicklung bei $p=2$ und $N_{1}=48$ Ständernuten zur Veranschaulichung einiger Effekte dienen.

\subsection{Variation der Läufergeometrie}

Im Folgenden sollen einige Beispiele für die Variation der Läufergeometrie gezeigt werden, wobei zunächst eine theoretische Betrachtung bezüglich der Wahl der Läufernutbreiten durchgeführt werden soll.

\subsubsection{Wahl der Läufernutbreiten}

Wird die Position von zunächst geometrisch gleichen Läufernuten über dem Umfang variiert, also moduliert, so ergibt sich für die infinitesimal schmale Darstellung der Nuten, wie sie in Abschnitt 3.6.5 eingeführt wurde, eine Stabwiderstandsverteilung $R_{\mathrm{s}}^{\prime}\left(x_{2}\right)$ mit einem minimalen Widerstand an der Stelle der am dichtesten liegenden Nuten und einem maximalen Widerstand an der Stelle des größten Nutabstands. Folglich beeinflusst die Variation der Nutpositionen über die Stabwiderstände den Ringstrom und somit letztendlich die Läuferrückwirkung.

Es lässt sich leicht nachvollziehen, dass sich bei einer beispielhaften sinusförmigen Anordnung der Nuten mit $b_{2}$ Urwicklungen über dem Umfang eine ebenfalls sinusförmige Widerstands-, aber auch Streuinduktivitätsmodulation der Stabwerte ergibt. Mit (3.112) auf Seite 37wird daraus ersichtlich, dass sich aus jeder ständerseitigen Feldwelle der räumlichen Ordnungszahl $\mu$, bei zunächst Vernachlässigung des Einkopplungsfaktors $\xi_{\text {u }}$ gemäß 


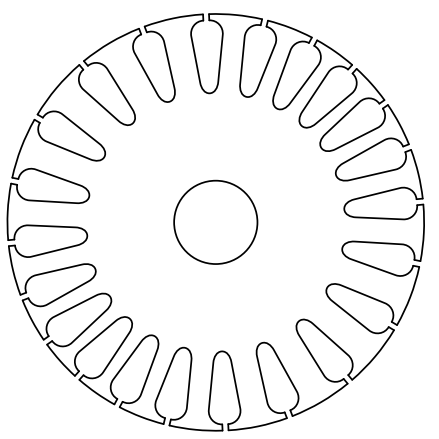

(a) Ohne Anpassung der Nutbreiten

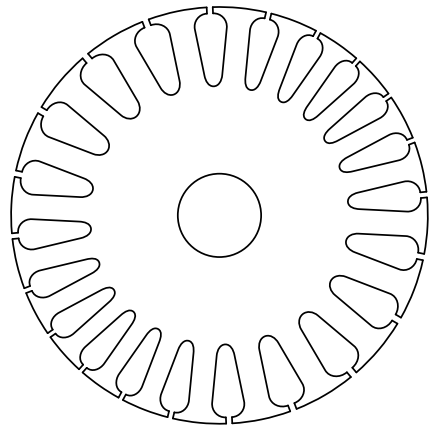

(b) Mit Anpassung der Nutbreiten; im Bereich kleinerer Nutabstände sind die Nuten schmaler, im Bereich größerer Abstände breiter

Bild 7.1: Läuferschnitt mit sinusförmiger Anordnung der Nuten

(3.61) auf Seite [37, Ringstromwellen der räumlichen Ordnungszahlen

$$
\nu=\mu \pm g \cdot b_{2} \text { mit } g=\{0,1,2, \ldots\}
$$

ausbilden. Folglich ergeben sich auch Induktionswellen mit denselben räumlichen Ordnungszahlen $\nu$, wie dies zum Beispiel in Tabelle 5.4 ersichtlich ist. Es ist zu beachten, dass (7.1) für die Betrachtung in läuferfesten Koordinaten gültig ist. Im Beispiel wird daher aus der räumlichen Ordnungszahl $\nu=2-1 \cdot 6=-4$ nach der Umrechnung in ständerfeste Koordinaten $\mu=4$.

Da sich die Gleichkomponenten von $R_{\mathrm{s}}^{\prime}$ und $L_{\sigma \mathrm{s}}^{\prime}$ bei einer ideal sinusförmigen Nutanordnung nicht ändern, handelt es sich bei den Ringstromwellen um zusätzliche Wellen. Es ist somit anzunehmen, dass eine komplette Kompensation der zusätzlichen Läuferstromwellen, insbesondere aufgrund der Ständerinduktionshauptwelle, zu geringeren unerwünschten Parasitäreffekten führt.

Es liegt folglich nahe, eine Kompensation der genannten Effekte anzustreben, indem die Nutbreiten bei einer Modulation der Läufernutpositionen nicht, wie in Bild 7.1a dargestellt, konstant gehalten werden, sondern, wie in Bild $7.1 \mathrm{~b}$ dargestellt, proportional zum Abstand der Nuten gewählt werden, sodass sich bei kontinuierlicher Betrachtung des Läuferkäfigs eine über der Winkelkoordinate konstante Stabwiderstandsverteilung $R_{\mathrm{s}}^{\prime}\left(x_{2}\right)=$ konst. ergibt. Es erweist sich zudem als vorteilhaft, die einzelnen Nuten unsymmetrisch auszuführen, sodass sich resultierend, wie in Bild 7.5b gut zu erkennen, symmetrische Zähne ergeben. Würden umgekehrt die Nuten symmetrisch ausgeführt, so ergäben sich bei stark unterschiedlichen benachbarten Nutabständen teilweise unterproportional schmale Zähne, welche den magnetischen Fluss nicht mehr ausreichend führen könnten.

Dieselbe Kompensation wie für den Einfluss auf die Stabwiderstandsverteilung $R_{\mathrm{s}}^{\prime}\left(x_{2}\right)$ bietet sich bei Betrachtung von (3.77) für die in Abschnitt 3.6.2 hergeleitete Stabstreuinduktivität beziehungsweise deren Verteilung $L_{\mathrm{s} \sigma}^{\prime}\left(x_{2}\right)$ an, die ihr Minimum bei nichtbreitenskalierter Nutgeometrie ebenfalls bei der maximalen Dichte und ihr Maximum bei der minimalen Dichte der Läuferstäbe besitzt. Hierbei sei einschränkend - wie bereits in Abschnitt 3.6.2 - angemerkt, dass die strenge umgekehrte Proportionalität der Stabstreuinduktivität zur Nutbreite $L_{\mathrm{s} \sigma} \sim 1 / b_{\mathrm{N}}$ nur für ausschließlich parallel zur Winkelkoordinate 
$x_{2}$ verlaufende Nutquerfelder gilt. Folglich ergibt sich im Allgemeinen für die im vorhergehenden Absatz vorgeschlagene Nutbreitenanpassung eine nur annähernd konstante Stabstreuinduktivität $L_{\mathrm{s} \sigma}^{\prime}\left(x_{2}\right) \approx$ konst. über dem Umfang.

Als Nebeneffekt der Nutbreitenanpassung werden zusätzlich durch die Modulation der Nutpositionen entstehende Luftspaltleitwertwellen, welche zu einem Reluktanzdrehmoment führen könnten, weitgehend unterdrückt.

Es sei angemerkt, dass die vorgeschlagene Kompensation der Auswirkung einer Variation der Läufernutpositionen auf die Stabwiderstands- und Stabstreuinduktivitätsverteilung zu unterschiedlichen Nutgeometrien innerhalb des Läufers führt. Für Maschinen, deren Läuferbleche mit Hilfe eines Komplettschnitts oder Folgeschnitts hergestellt werden, ist dies weitgehend kostenneutral. Beim Tippschneiden wird hingegen jede Nut einzeln mit demselben Werkzeug geschnitten [31]. Für das Einbringen beliebig angeordneter aber gleichförmiger Nuten ist dieses Verfahren folglich hervorragend geeignet, die Fertigung verschiedener Nutformen innerhalb eines Blechs würde jedoch den Einsatz verschiedener Stanzwerkzeuge mit entsprechenden Kosten erfordern.

\subsubsection{Einbeziehung von Nichtlinearitäten}

Die in Abschnitt 7.3.1 beschriebenen Wahl der Läufernutbreiten führt zu einer Homogenisierung des Käfigs über dem Umfang und damit zu einer gewissen Vereinheitlichung der Rückwirkung aller Käfigmaschen auf die Ständerinduktionshauptwelle.

Zur mathematischen Beschreibung dieser Rückwirkung sei die Ständerinduktionshauptwelle durch den komplexen Raumzeiger $\underline{b}_{1 p}$ dargestellt. Die von einer Masche $n$ erregte auf $\underline{b}_{1 p}$ rückwirkende Induktionswelle mit der räumlichen Ordnungszahl $\nu=p$ lässt sich ebenfalls durch einen komplexen Raumzeiger $\underline{b}_{2 p(n)}$ beschreiben. Mit dieser Darstellung ergäbe sich die optimal vereinheitlichte Rückwirkung aller Käfigmaschen, wenn für alle $N_{2}$ Käfigmaschen

$$
\frac{\underline{b}_{2 p(n)}}{\underline{b}_{1 p} \cdot\left(x_{(n+1)}-x_{(n)}\right)}=\underline{k}
$$

gilt. Dabei ist $\underline{k}$ ein für alle Käfigmaschen konstanter Faktor, und $x_{(n)}$ sowie $x_{(n+1)}$ sind die der Masche zugehörigen Nutpositionen.

Insbesondere bei Einbeziehung der zahn- und nutbreitenabhängigen Nichtlinearitäten, welche sich zum Beispiel für die Stabstreuinduktivitäten $L_{\mathrm{s} \sigma(n)}$ und mit dem Einkopplungsfaktor $\xi_{\mathrm{u}}$ gemäß (3.61) für die induzierten Spannungen $\underline{u}_{\mathrm{i} 1 p(n)}$ ergeben, ist die Bedingung (7.2) durch ausschließliche Anpassung der Läufernutbreiten nicht erfüllbar. Dies wird nach Aufstellung einer Matrizengleichung zur Berechnung des Verhältnisses von $\underline{b}_{2 p(n)}$ zu $\underline{b}_{1 p} \cdot\left(x_{(n+1)}-x_{(n)}\right)$ unter Verwendung von (3.99) schnell ersichtlich.

Es liegt daher nahe, die Nutbreiten der Forderung (7.2) mittels numerischer Optimierung bestmöglich anzunähern. Numerische Untersuchungen an einigen beispielhaft ausgewählten Maschinen zeigen dabei, dass diese Optimierung, also die Minimierung der Differenzen der einzelnen komplexen Faktoren zwischen $\underline{b}_{2 p(n)}$ und $\underline{b}_{1 p} \cdot\left(x_{(n+1)}-x_{(n)}\right)$, gegenüber der einfacheren Wahl der Nutbreiten gemäß Abschnitt 7.3.1 lediglich zu Unterschieden bezüglich der Eigenschaften der Maschinen im Bereich der numerischen Rechengenauigkeit führt. Es soll daher im folgenden auf diese Optimierung verzichtet werden. 


\subsubsection{Maschine „B“}

Maschine „B“ verfügt über einen Kupferdruckgussläufer, wodurch sich fertigungsbedingt signifikante Einschränkungen bezüglich der herstellbaren Nutgeometrien ergeben. Bei Anwendung der in Abschnitt 7.3.1 vorgeschlagenen Nutbreitenanpassung ergibt sich daraus insbesondere eine deutliche Einschränkung der ausführbaren Nutabstände und somit eine verringerte Einflussmöglichkeit auf das Geräusch oder andere Betriebseigenschaften der Maschine.

Zwecks Analyse soll zunächst die Maschine mit regelmäßig genutetem Läufer betrachtet werden. Hier, aber auch bei der nachfolgend gezeigten Variante mit modulierten Läufernutpositionen, kann aufgrund der ungeraden Zahl von $N_{2}=33$ Läufernuten erwartet werden, dass sich eine Rüttelkraft, also eine Jochzugwelle mit der räumlichen Ordnungszahl $|\nu|=1$ ausbildet.

Die Analyse des Geräuschs, dessen Spektrum bei einer minimalen Drehzahlabweichung für den bei $n=730 \mathrm{~min}^{-1}$ liegenden Bemessungspunkt in Bild 7.2a dargestellt ist, zeigt gerade eine derartige Komponente, nämlich mit der räumlichen Ordnungszahl $\nu=-1$, als dominant. Es handelt sich um eine Welle mit der Frequenz $f=501,5 \mathrm{~Hz}$, wie sie sich zum Beispiel aus der nutharmonischen Ständerinduktionswelle mit der räumlichen Ordnungszahl $\mu=-32$ in Kombination mit der nutharmonischen Läuferinduktionswelle der räumliche Ordnungszahl $\nu=31$ ergibt, die sich als Rückwirkung auf die Ständerinduktionswelle mit $\tilde{\mu}=-2$ ausbildet. Die Ordnungszahl $\tilde{\mu}=-2$ ergibt sich dabei gerade mit $g=-1$ aus (3.13).

Es sei angemerkt, dass die in GRION verwendete Routine zur Berechnung der Schallleistung die Auswirkung einer Rüttelkraft modellbedingt zu niedrig abbildet. Zur Kompensation dieser Tatsache wurde anhand der Messwerte des unmodulierten Läufers eine empirische Erhöhung aller durch Rüttelkräfte hervorgerufener Komponenten im Geräusch der Maschine um $28 \mathrm{~dB}$ durchgeführt.

Mit Hilfe einer numerischen Optimierung der analytisch berechneten Maschineneigenschaften, wurde mit dem Ziel einer möglichst geringen Geräuschentwicklung bei geringen Pendeldrehmomenten und ausreichend großen Nut- und Zahnquerschnitten ein alternativer Läufer mit modulierten Nutpositionen entwickelt. Die resultierende Geometrie, welche am Umfang drei gleiche Blöcke von je 11 Nuten enthält, ist in Bild 7.3b zu erkennen. Die Wahl gleicher Blöcke zielt dabei insbesondere, wie bereits in Kapitel 1 am Beispiel von Ventilatoren gezeigt, auf die Unterdrückung von mechanischen Unwuchten ab, welche zwar durch die Anpassung der Läufernutbreiten bereits beschränkt, aber nicht unbedingt vollständig unterdrückt werden.

Ein Vergleich der in Bild 7.2 dargestellten Spektren des Geräuschs sowohl der Maschine mit modulierten Läufernuten als auch der Maschine ohne modulierte Läufernuten zeigt, dass die erfolgte Optimierung der Läufernutpositionen insbesondere in Bezug auf einen Einzelton bei etwa $430 \mathrm{~Hz}$ nicht erfolgreich war. Dies ist darin begründet, dass der genannte Einzelton, welcher mit einer Jochzugwelle der Ordnungszahl $|\nu|=1$ einhergeht, auf einer relativ kleinen Induktionswelle beruht, welche aufgrund der gewählten Berechnungsgenauigkeit zum Zeitpunkt der Optimierung der Maschinengeometrie nicht im Berechnungsergebnis enthalten war. 


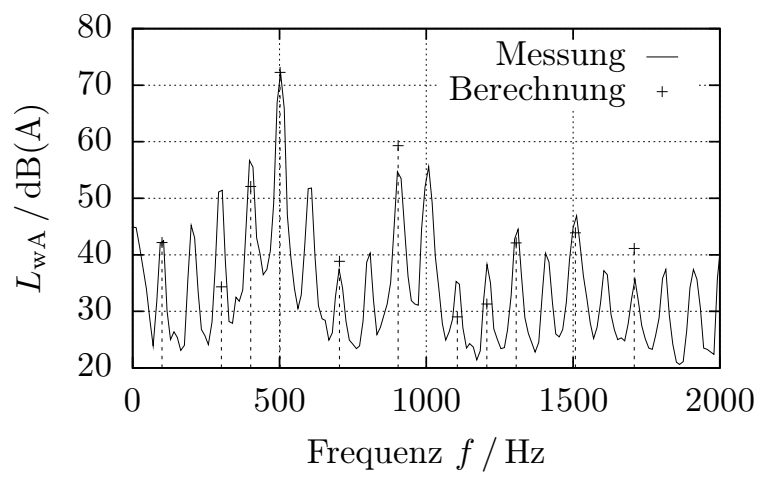

(a) Unmodulierter Läufer $-n=733 \min ^{-1}$

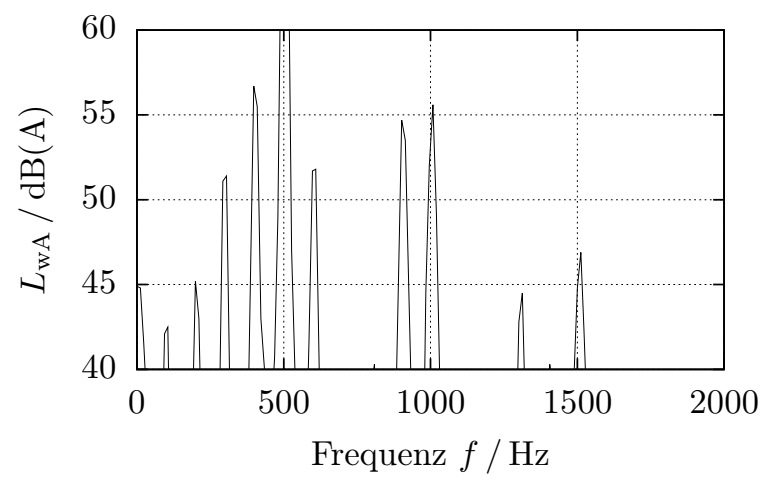

(c) Unmodulierter Läufer - Ausschnittvergrößerung der Messung

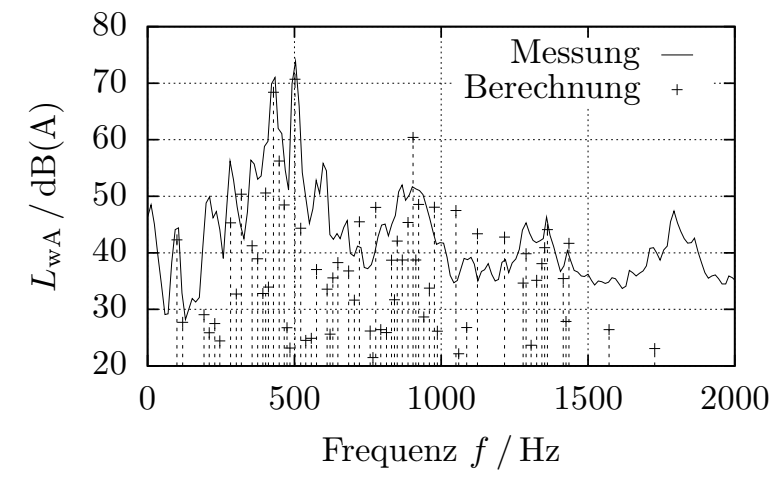

(b) Modulierter Läufer $-n=731 \mathrm{~min}^{-1}$

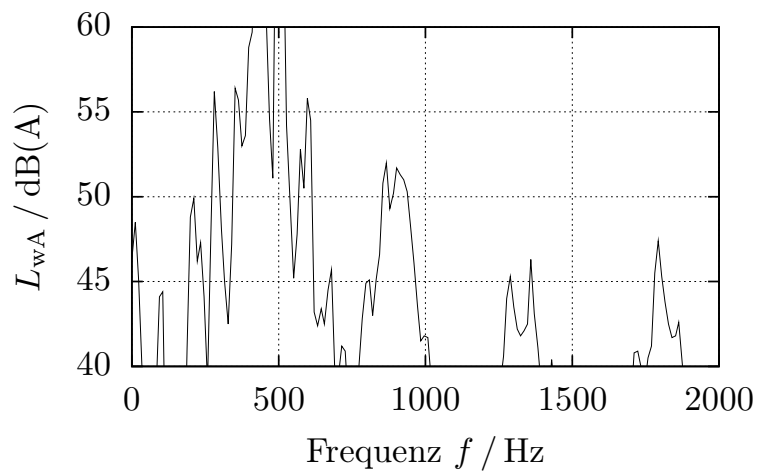

(d) Modulierter Läufer - Ausschnittvergrößerung der Messung

Bild 7.2: Bewerteter Schalleistungspegel $L_{\mathrm{wA}}(f)$ von Maschine „B“

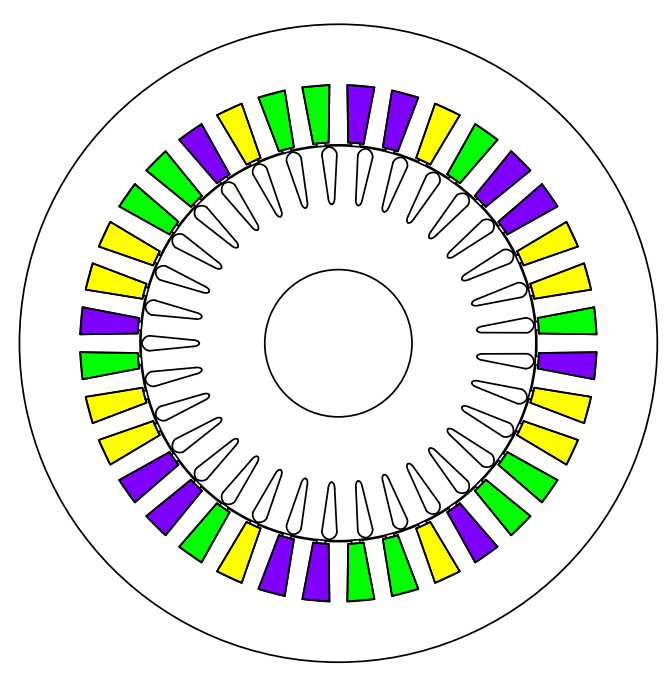

(a) Unmodulierter Läufer

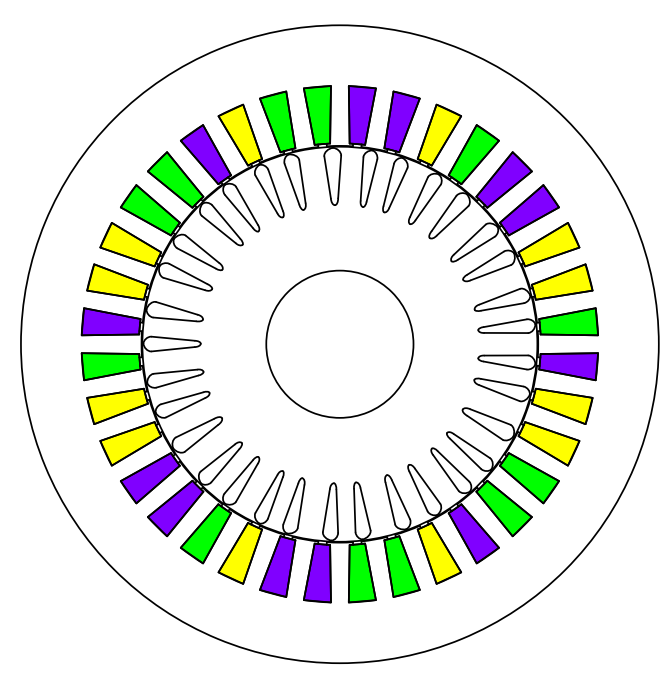

(b) Modulierter Läufer

Bild 7.3: Maschine „B“ mit $N_{2}=33$ Läufernuten 
Die Nachrechnung der Maschine mit erhöhter Rechengenauigkeit, also bei Berücksichtigung von Feldwellen mit niedrigeren Amplituden, liefert, wie in Bild 7.2b ersichtlich, die fehlende Komponente bei etwa $430 \mathrm{~Hz}$. Eine Optimierung mit dieser Rechengenauigkeit führt zwar zu einer zu Bild 7.3b verschiedenen Geometrie, es konnte aber keine gegenüber der Maschine mit regelmäßig genutetem Läufer deutlich leiser wirkende Geometrie gefunden werden. Dies liegt nicht zuletzt an der ungeraden Läufernutzahl, welche schnell zu einseitig magnetischen Zügen führt, sowie an den fertigungsbedingten Einschränkungen der ausführbaren Nutgeometrien.

Der Vergleich der Spektren in Bild 7.2 zeigt auch bei der angewandten höheren Rechengenauigkeit, dass die Schallleistung des lautesten Einzeltons, nämlich dessen mit $f=501,5 \mathrm{~Hz}$, bei moduliertem Läufer rechnerisch zwar um etwa $2 \mathrm{~dB}(\mathrm{~A})$ niedriger liegt, sich dies in der Messung jedoch nicht widerspiegelt. Folglich liegt diese Differenz bereits im Bereich der Rechen- beziehungsweise Messungenauigkeit.

Zusammenfassend zeigt sich eine zufriedenstellende Übereinstimmung der berechneten mit den gemessenen Schallleistungen. Eine exakte Übereinstimmung wäre dabei aufgrund der Modellvereinfachungen des mechanischen Aufbaus der Maschine nicht zu erwarten. Es lässt sich damit annehmen, dass die Berechnung der Schallleistung mit Hilfe des in dieser Arbeit vorgestellten Verfahrens zur Optimierung von elektrischen Maschinen geeignet, eine Verifikation am Prototypen aber unabdingbar ist.

\subsubsection{Maschine „C“}

Für Maschine „C“ werden im Folgenden verschiedene Läufer betrachtet.

\subsubsection{1 $N_{2}=40$ Nuten}

In Bild 7.4a ist sowohl der berechnete als auch der gemessene A-bewertete Schallleistungspegel von Maschine „C“ gemäß Bild [7.5a, also mit einem Läufer, welcher $N_{2}=40$ gleichmäßig angeordnete Nuten besitzt, dargestellt. Die Werte gelten dabei für die Drehzahl $n=1475 \mathrm{~min}^{-1}$ und den Ständerstrangstrom $I_{1}=16,3 \mathrm{~A}$. Im berechneten Schallleistungspegel sind neben der doppelten Netzfrequenz unter anderem zwei deutliche Frequenzanteile bei $f=983, \overline{3} \mathrm{~Hz}$ und $f=1083, \overline{3} \mathrm{~Hz}$ zu erkennen, wie sie sich zum Beispiel aus dem Zusammenspiel der ständer- und läufernutharmonischen Luftspaltinduktionswellen ergeben. Die Messung enthält jedoch noch weitere deutliche Spitzen wie zum Beispiel bei etwa $f=883 \mathrm{~Hz}, f=1183 \mathrm{~Hz}$ und $f=2070 \mathrm{~Hz}$. Hierbei sind die erste und dritte Frequenz zwar im berechneten Spektrum enthalten, jedoch mit deutlich geringeren Amplituden. Die Betrachtung der in Tabelle 7.2 dargestellten Pendeldrehmomente, die sich aus der Berechnung gemäß Kapitel 3 und Kapitel 4 mit Hilfe des Berechnungsprogramms GRION ergeben, zeigt, dass dort gerade Komponenten mit diesen beiden Frequenzen enthalten sind. Es lässt sich daher vermuten, dass die beiden Einzeltöne gerade auf der Wirkung der Pendeldrehmomente beruhen, welche bei der Geräuchberechnung nicht berücksichtigt sind. Eine Geräuschanregung mit etwa $f=1183 \mathrm{~Hz}$ ist hingegen nicht in den Berechnungsergebnissen enthalten, was sich mit der angewandten Vereinfachung der Vernachlässigung der sättigungsbedingten Leitwertwellen gemäß (6.1), die in Abschnitt 3.4.2 beschrieben sind, erklären lässt. Würde die genannte Vereinfachung aufgehoben, so ergäbe sich ein 


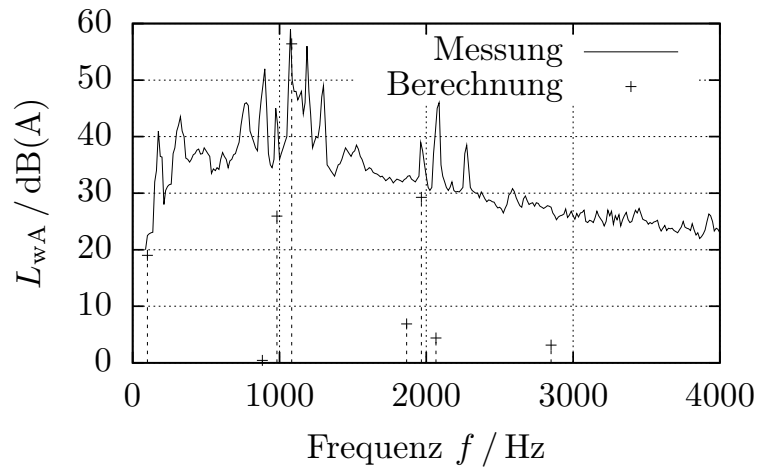

(a) Unmodulierter Läufer; berechnete und gemessene Werte

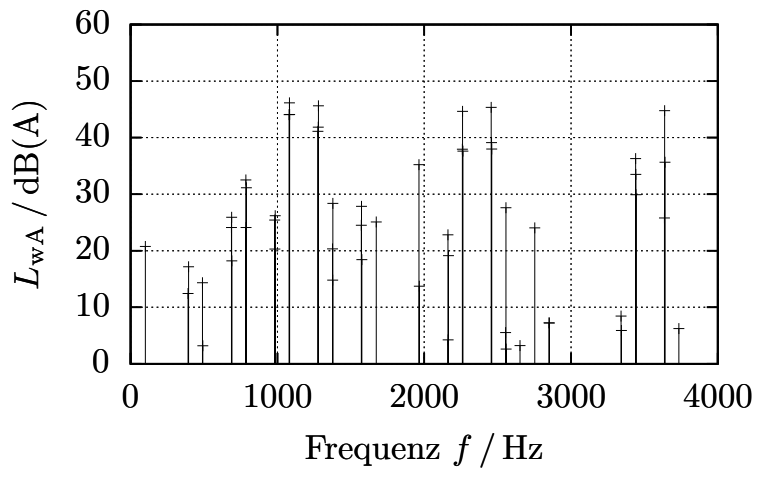

(b) In vier Blöcken gemäß Bild $7.5 \mathrm{~b}$ modulierter Läufer; berechnete Werte

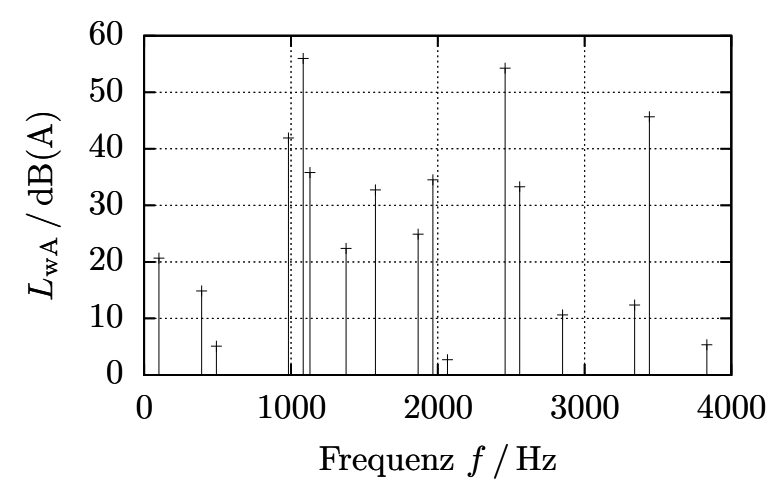

(c) In zehn Blöcken gemäß Bild $7.5 \mathrm{~d}$ modulierter Läufer; berechnete Werte

Bild 7.4: A-bewerteter Schalleistungspegel $L_{\mathrm{wA}}(f)$ von Maschine „C" mit $N_{2}=40$-nutigem Läufer bei der Drehzahl $n=1475 \mathrm{~min}^{-1}$ und dem Strangstromeffektivwert $I_{1}=16,3 \mathrm{~A}$

zusätzlicher Einzelton bei $f=1183, \overline{3} \mathrm{~Hz}$, welcher um die in (6.1) enthaltene doppelte Ständerfrequenz $f_{1}$ oberhalb der in Bild 7.4a enthaltenen Komponente mit $f=1083, \overline{3} \mathrm{~Hz}$ liegen würde.

Modulation in 4 Blöcken Um einen modulationsbedingten einseitigen magnetischen Zug zu verhindern, ist es notwendig, die Nutpositionen mit einer geradzahligen Periodizität zu wählen. Für einen Läufer mit $N_{2}=40$ Nuten bedeutet dies, dass die Nuten in 2, 4, 8, 10 oder 20 gleichen Blöcken mit je 20, 10, 5, 4 oder 2 Nuten angeordnet werden können. Dabei soll im Folgenden zunächst eine Modulation mit 4 Blöcken betrachtet werden, wie diese in Bild 7.5b dargestellt ist.

Aufgrund der $N_{1}=48$ Ständer- und $N_{2}=40$ Läufernuten und der $p=2$ Polpaare bilden sich ohne Modulation sowohl ständer- als auch läuferseitig ausschließlich Induktionswellen der räumlichen Ordnungszahlen

$$
\nu_{\mathrm{B}}=2+k \cdot 4 \text { mit } k \in \mathbb{Z}
$$

aus. Folglich ergeben sich ausschließlich Radialzugwellen und aufgrund der zu vier vielfachen Ständernutzahl $N_{1}=48$ somit auch Jochzugwellen der räumlichen Ordnungszahlen

$$
\nu_{\sigma}=k \cdot 4 \text { mit } k \in \mathbb{Z} .
$$




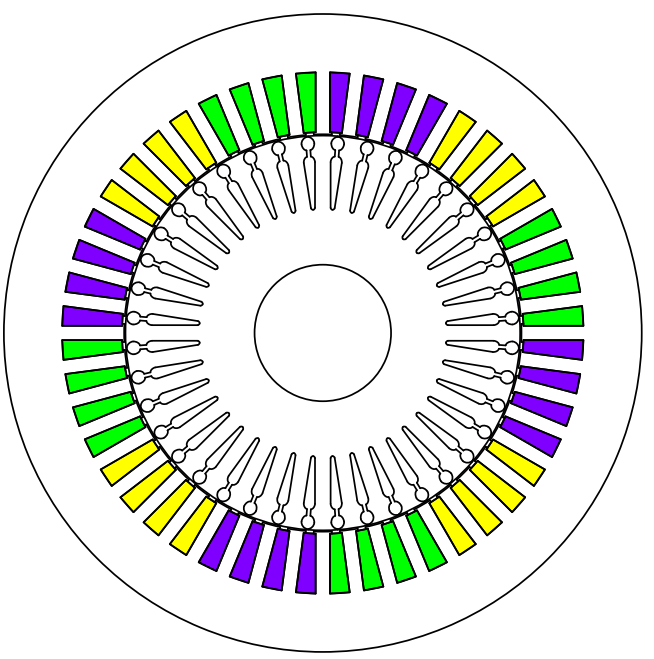

(a) Unmodulierter Läufer

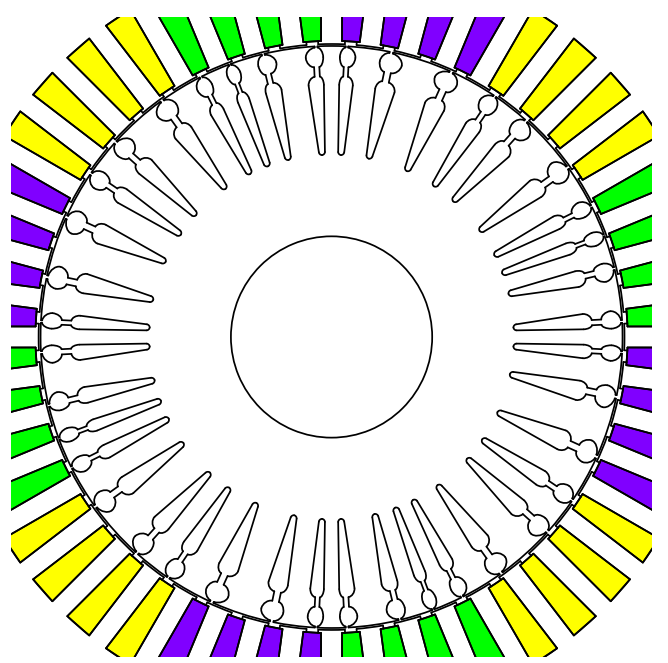

(b) In vier Blöcken modulierter Läufer mit unterschiedlichen Nutformen

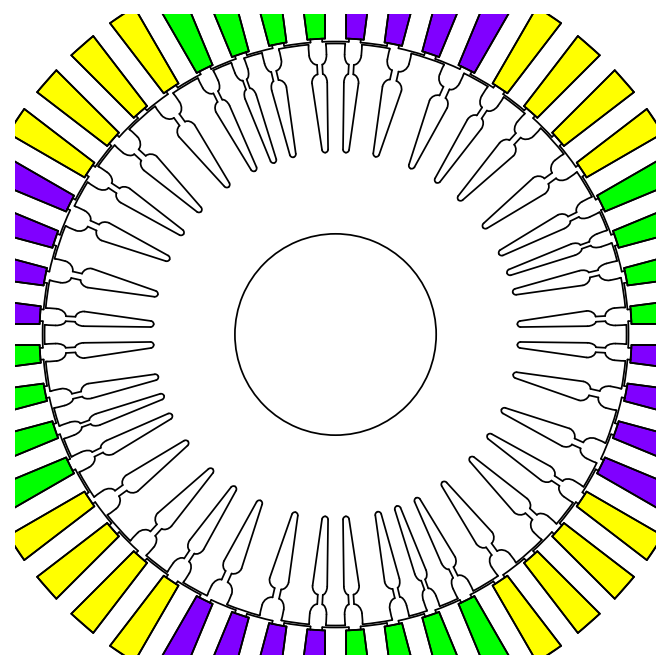

(d) In vier Blöcken modulierter Läufer mit unterschiedlichen Nutformen und weiten Nutschlitzen

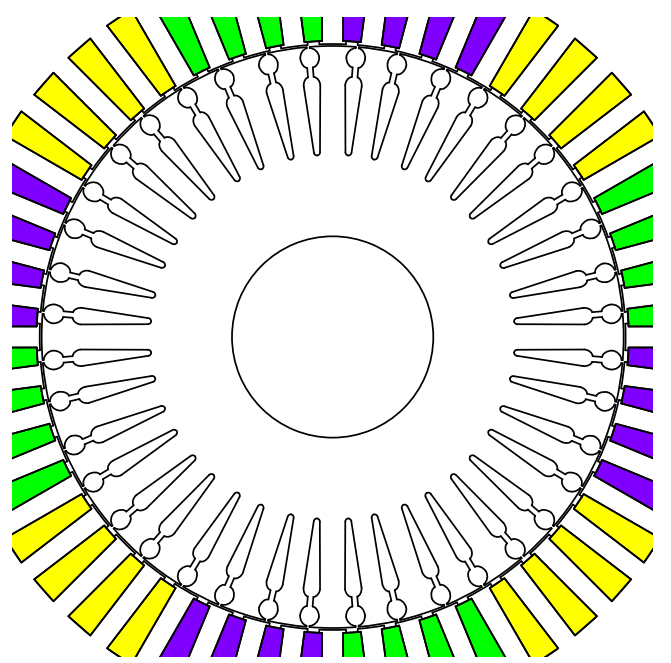

(c) In zehn Blöcken modulierter Läufer mit unterschiedlichen Nutformen

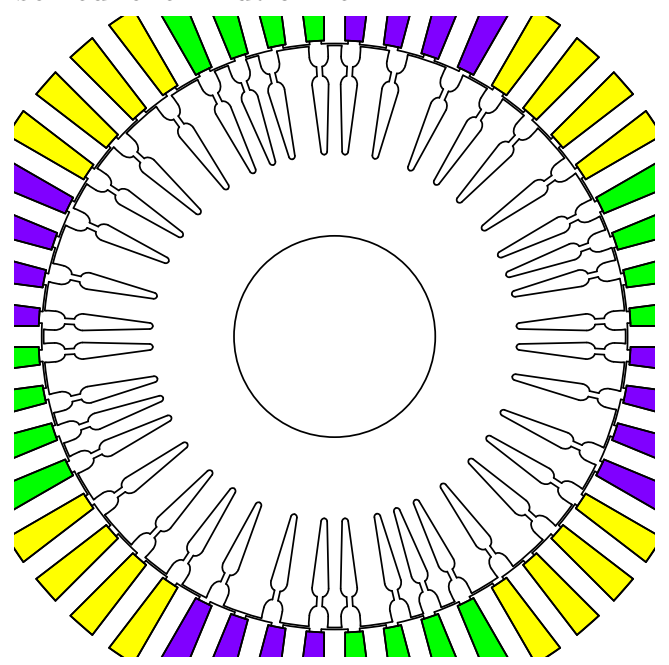

(e) In vier Blöcken modulierter Läufer mit gleichen Nutformen und weiten Nutschlitzen

Bild 7.5: Maschine „C" mit $N_{2}=40$ Läufernuten 
Tabelle 7.2: Berechnetes Drehmoment von Maschine „C" mit herkömmlichem $N_{2}=40$-nutigem Läufer bei der Drehzahl $n=1475 \mathrm{~min}^{-1}$ und dem Strangstromeffektivwert $I_{1}=16,3 \mathrm{~A}$

\begin{tabular}{rrr}
\hline$M /[\mathrm{Nm}]$ & $f /[\mathrm{Hz}]$ & $\varphi /\left[^{\circ}\right]$ \\
\hline 117,22 & 0,00 & 0,0 \\
3,01 & 883,33 & 102,8 \\
0,27 & 2066,67 & 83,7 \\
0,09 & 5900,00 & 129,2 \\
0,06 & 3833,33 & 117,1 \\
0,05 & 2950,00 & $-67,5$ \\
\hline
\end{tabular}

Dieser Zusammenhang, der zu einer Beschränkung der maximalen relativen Schallleistung gemäß Bild 4.5 führt, bleibt sowohl bei der Modulation mit 4, als auch mit 8 Blöcken erhalten. Aus diesem Grund erscheint die Wahl von 4 Blöcken gegenüber 2, 10 oder 20 Blöcken vorteilhaft.

Es ist jedoch bei Modulation mit 4 Blöcken zu erwarten, dass sich eine Leitwertwelle der räumlichen Ordnungszahl $\nu=4$ mit der vierfachen Winkelgeschwindigkeit des Läufers, also eine Leitwertwelle der Form

$$
\lambda_{\text {mod }}\left(x_{1}, t\right)=\hat{\lambda}_{\text {mod }} \cdot \cos \left(4 x_{1}-4 \Omega t+\varphi\right)
$$

ausbildet. Aufgrund der Vierpoligkeit des Motors würde sich hieraus ein Reluktanzdrehmoment entwickeln, welches sich bei einem Schlupf von $s=0$ konstant darstellt und andernfalls als Pendeldrehmoment mit der schlupfabhängigen Kreisfrequenz

$$
\omega_{\mathrm{Mmod}}=2 \cdot s \cdot \omega_{1}
$$

in Erscheinung tritt.

Um diesen Effekt deutlich aufzuzeigen, wurde beispielhaft eine Berechnung mit einem Läufer mit vergrößerten Nutschlitzen durchgeführt, wie er in Bild $7.5 \mathrm{~d}$ zu sehen ist.

Für das betrachtete Beispiel ergibt sich entsprechend Tabelle 7.3 eine Leitwertwelle gemäß (17.5), deren Amplitude bei etwa 0,33\% des konstanten Anteils des Luftspaltleitwerts liegt. Das Drehmoment mit der Kreisfrequenz gemäß (7.6), welches sich nicht ausschließlich als Reluktanzdrehmoment darstellt, liegt, wie in Tabelle 7.4 ersichtlich, bei etwa 0,68\% des zeitlich invarianten Drehmomentanteils. Diese für die gewählte Nutzschlitzbreite relativ geringen Werte ergeben sich dabei aus der Anwendung der in Abschnitt 7.3.1 vorgeschlagenen Nutbreitenanpassung. Hierdurch werden sowohl die Widerstands- sowie Streuinduktivitätsverteilungen, als auch der Luftspaltleitwert, welcher aufgrund der Läufernutung entsteht, über dem Umfang vergleichmäßigt.

Wird die Nutbreitenanpassung nicht angewendet, womit sich zum Beispiel eine Geometrie gemäß Bild 7.5e ergibt, so ist die Leitwertwelle gemäß (7.5), wie in Tabelle 7.5 ersichtlich, mit 5,9\% gegenüber dem Gleichanteil etwa um den Faktor 9 größer. Gleichzeitig entsteht auch ein größeres Pendeldrehmoment, dessen Amplitude gemäß Tabelle [7.6 etwa 8,3\% des Gleichanteils ausmacht.

Ein zweiter Effekt, welcher bei Modulation mit 4 Blöcken auftritt, lässt sich durch die zusätzlichen quasinutharmonischen Felderregerwellen der Läuferwicklung erklären, die sich 
Tabelle 7.3: Leitwertwellen von Maschine „C“ mit breiten Läufernutschlitzen bei angepasster Nutbreite und $n=1475 \mathrm{~min}^{-1}$

\begin{tabular}{rrrrrr}
\hline$\hat{\lambda} / \frac{\mu_{0}}{\delta}$ & $\nu$ & $\nu / p$ & $f /[\mathrm{Hz}]$ & $\sigma /\left[^{\circ}\right]$ & $\varphi /\left[^{\circ}\right]$ \\
\hline 0,61291 & 0 & 0 & 0,00 & 0,0 & 0,0 \\
0,19760 & 48 & 24 & 0,00 & 0,0 & 0,0 \\
0,19743 & 36 & 18 & 885,00 & 270,0 & $-47,0$ \\
$\vdots$ & $\vdots$ & $\vdots$ & $\vdots$ & $\vdots$ & $\vdots$ \\
0,00209 & 4 & 2 & 98,33 & 30,0 & 128,6 \\
$\vdots$ & $\vdots$ & $\vdots$ & $\vdots$ & $\vdots$ & $\vdots$ \\
\hline
\end{tabular}

Tabelle 7.4: Drehmoment von Maschine „C" mit breiten Läufernutschlitzen bei angepasster Nutbreite und $n=1475 \mathrm{~min}^{-1}$

\begin{tabular}{rrr}
\hline$M /[\mathrm{Nm}]$ & $f /[\mathrm{Hz}]$ & $\varphi /\left[^{\circ}\right]$ \\
\hline 117,12 & 0,00 & 0,0 \\
$\vdots$ & $\vdots$ & $\vdots$ \\
0,80 & 1,67 & 140,2 \\
$\vdots$ & $\vdots$ & $\vdots$ \\
\hline
\end{tabular}

Tabelle 7.5: Leitwertwellen von Maschine „C“ mit einheitlichen Läufernutgeometrien und breiten Läufernutschlitzen bei $n=1475 \mathrm{~min}^{-1}$

\begin{tabular}{rrrrrr}
\hline$\hat{\lambda} / \frac{\mu_{0}}{\delta}$ & $\nu$ & $\nu / p$ & $f /[\mathrm{Hz}]$ & $\sigma /\left[^{\circ}\right]$ & $\varphi /\left[^{\circ}\right]$ \\
\hline 0,61291 & 0 & 0 & 0,00 & 0,0 & 0,0 \\
0,19760 & 48 & 24 & 0,00 & 0,0 & 0,0 \\
0,18690 & 36 & 18 & 885,00 & 270,0 & $-47,5$ \\
$\vdots$ & $\vdots$ & $\vdots$ & $\vdots$ & $\vdots$ & $\vdots$ \\
0,03643 & 4 & 2 & 98,33 & 30,0 & 127,1 \\
$\vdots$ & $\vdots$ & $\vdots$ & $\vdots$ & $\vdots$ & $\vdots$ \\
\hline
\end{tabular}

Tabelle 7.6: Drehmoment von Maschine „C“ mit gleichen Läufernuten und breiten Läufernutschlitzen bei $n=1475 \mathrm{~min}^{-1}$

\begin{tabular}{rrr}
\hline$M /[\mathrm{Nm}]$ & $f /[\mathrm{Hz}]$ & $\varphi /\left[^{\circ}\right]$ \\
\hline 116,96 & 0,00 & 0,0 \\
9,73 & 1,67 & 173,1 \\
$\vdots$ & $\vdots$ & $\vdots$ \\
\hline
\end{tabular}


aufgrund der Modulation der Nutpositionen ergeben.

Für gleichmäßig genutete Maschinen lassen sich die räumlichen Ordnungszahlen der nutharmonischen Felderregerwellen des Läufers gemäß (3.127) bestimmen. Demnach ergeben sich zum Beispiel als Rückwirkung der Hauptwelle mit der räumlichen Ordnungszahl $\nu=p$ und der Kreisfrequenz $\omega_{1}$ unter anderem die Felderregerwellen

$$
V_{2, \nu}\left(x_{2}, t\right)=\hat{V}_{2, \nu} \cdot \cos \left(\nu x_{2}-\omega_{2} t+\varphi\right)
$$

mit den Ordnungszahlen $\nu=p=2, \nu=p-N_{2}=2-40=-38$ und $\nu=p+N_{2}=$ $2+40=42$.

Wird nun eine Modulation der Läufernutpositionen in $b_{2}=2 p=4$ Blöcken durchgeführt, so ergeben sich, ähnlich wie sich bei einer Frequenzmodulation neben der Trägerfrequenz die Seitenfrequenzen ergeben, zum Beispiel die zusätzlichen quasinutharmonischen Felderregerwellen der Läuferwicklung mit den räumlichen Ordnungszahlen $\nu=p-N_{2}-b_{2}=$ $2-40-4=-42$ und $\nu=p+N_{2}-b_{2}=2+40-4=38$.

Nach Überführung in das ständerfeste Koordinatensystem ergeben sich die beispielhaft genannten Felderregerwellen zu

$$
\begin{aligned}
& V_{2,2}\left(x_{1}, t\right)=\hat{V}_{2,2} \cdot \cos \left(2 \cdot x_{1}-\omega_{1} t+\varphi\right) \\
& V_{2,42}\left(x_{1}, t\right)=\hat{V}_{2,42} \cdot \cos \left(42 x_{1}-\left(40 \Omega+\omega_{1}\right) t+\varphi\right) \\
& V_{2,-42}\left(x_{1}, t\right)=\hat{V}_{2,-42} \cdot \cos \left(42 x_{1}-\left(44 \Omega-\omega_{1}\right) t+\varphi\right) \\
& V_{2,-38}\left(x_{1}, t\right)=\hat{V}_{2,-38} \cdot \cos \left(38 x_{1}-\left(40 \Omega-\omega_{1}\right) t+\varphi\right) \\
& V_{2,38}\left(x_{1}, t\right)=\hat{V}_{2,38} \cdot \cos \left(38 x_{1}-\left(36 \Omega+\omega_{1}\right) t+\varphi\right),
\end{aligned}
$$

sodass jeweils zwei nut- beziehungsweise quasinutharmonische Felderregerwellen mit betragsmäßig gleichen räumlichen Ordnungszahlen und um $\Delta \omega=p \cdot s \cdot \omega_{1}$ verschiedenen Kreisfrequenzen entstehen.

Aus den beispielhaft genannten Felderregerwellen (7.9) bis (17.12) entstehen wiederum Läuferinduktionswellen und in Kombination mit zum Beispiel der Ständerinduktionshauptwelle Radialzugwellen im Luftspalt. Die Ordnungszahlen dieser Radialzugwellen liegen dabei aufgrund der üblicherweise nahe beieinander liegenden Nutzahlen von Ständer und Läufer nahe der Ständernutzahl. Somit ergeben sich nach Modulation über die Ständerzähne als Resultat der genannten Felderregerwellen Jochzugwellen, die gemäß Bild 4.5 auf Seite 58 eine nicht vernachlässigbare relative Schallleistung besitzen und somit zu einem signifikanten Geräusch führen.

Es wird damit ersichtlich, dass sich bei üblichen Maschinengeometrien aus den in (7.9) bis (7.12) beispielhaft gezeigten Felderregerwellen der Läuferwicklung in der Regel je zwei Einzeltöne mit um $\Delta \omega$ verschiedener Kreisfrequenz ergeben. Diese je zwei Einzeltöne sind deutlich in dem in Bild 7.7a für die Geometrie gemäß Bild 7.5b, also mit schmaler Läufernutschlitzbreite, über der Drehzahl dargestellten Geräuschspektrum zu erkennen. Bei kleinerem Schlupf laufen diese Töne aufeinander zu, sodass sie als Schwebung im Geräusch in Erscheinung treten. Vom Menschen werden sie also nicht mehr als zwei, sondern als ein einzelner auf- und abschwellender Ton wahrgenommen, sodass leicht der Eindruck einer deutlichen Drehmomentpendelung entsteht. Folglich ist zu erwarten, dass die Akzeptanz einer derartigen Maschine im Kundenkreis fraglich ist. 


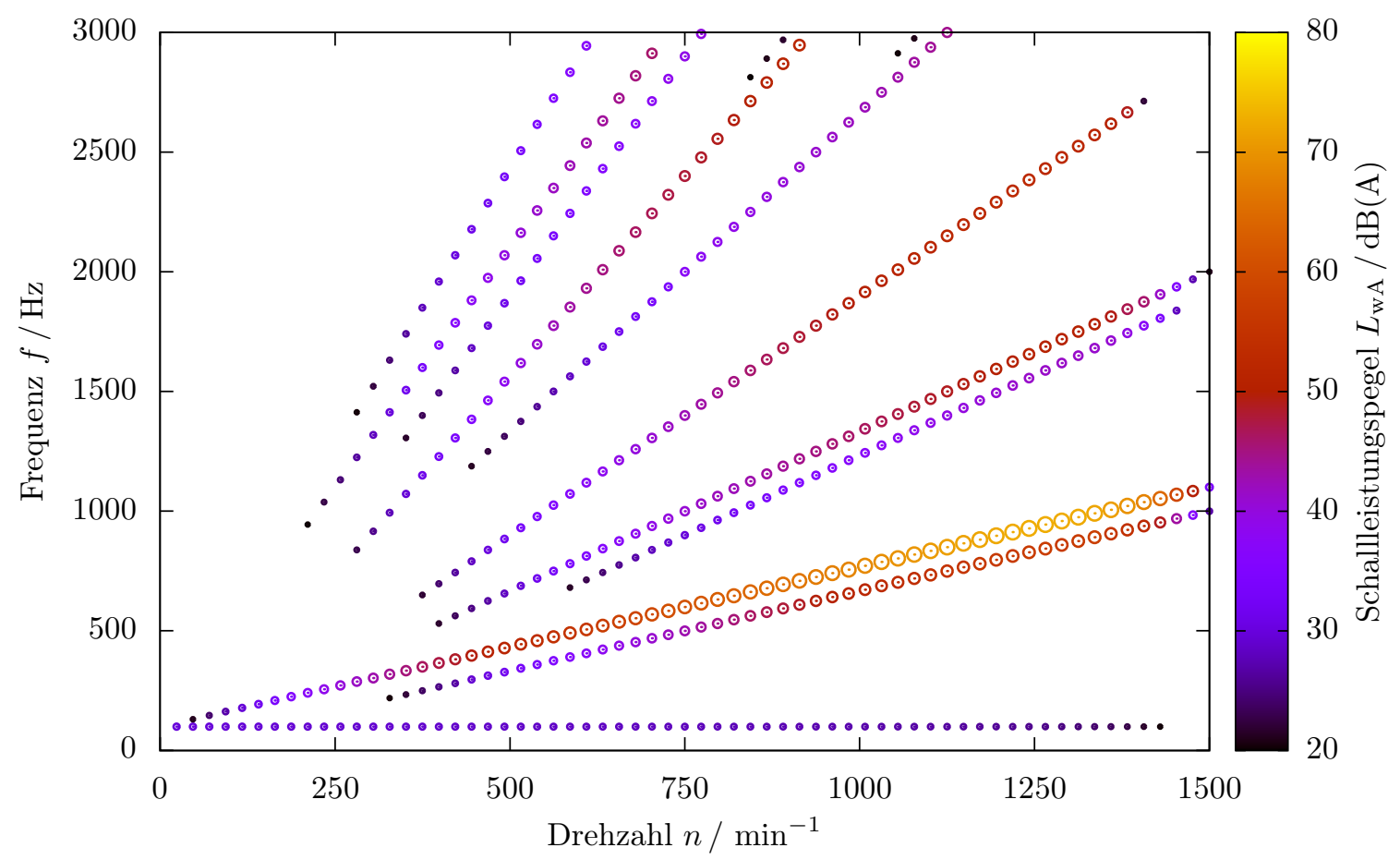

Bild 7.6: Schallleistung der Einzeltöne von Maschine „C“ mit $N_{2}=40$ gleichmäßig angeordneten Läufernuten bei Betrieb an $U_{1, \mathrm{str}}=398 \mathrm{~V}$ mit $f_{1}=50 \mathrm{~Hz}$ über der Drehzahl und Frequenz

Damit wird ersichtlich, dass die Wahl von $b_{2}=4$ Blöcken bei Maschine "C" mit $N_{2}=40$ Läufernuten nicht vorteilhaft ist, obwohl ein Vergleich der Spektren, welche in Bild 7.4a und Bild 7.4b beispielhaft für den Bemessungspunkt und in Bild [7.6] sowie Bild 7.7a für einen weiten Drehzahlbereich dargestellt sind, eine deutliche Reduktion der Schallleistungen der lautesten Einzeltöne verspricht.

Modulation in 10 Blöcken Um der bei Modulation in 4 Blöcken entstehenden Schwebung im Geräusch zu entgehen, soll ein Läufer betrachtet werden, dessen Nutpositionen in $b_{2}=10$ Blöcken moduliert sind.

Der Vorteil, dass sich bei der vorliegenden Kombination von Ständer- und Läufernutzahlen sowie der Polpaarzahl $p=2$ mit 4 Blöcken keine Jochzugwellen der räumlichen Ordnungszahl $\nu=2$ ausbilden können, entfällt bei der Variante mit 10 Blöcken. Das bedeutet, dass hierbei Jochzugwellen entstehen können, deren relative Schallleistung größer ist als bei der Variante mit 4 Blöcken. Zudem liegt die Eigenfrequenz der Schwingungsform $\nu=2$ des Ständers bei der betrachteten Maschine „C" mit $f_{\text {eigen }, 2} \approx 850 \mathrm{~Hz}$ in einem Bereich, welcher beim Anlauf der Maschine mit großer Wahrscheinlichkeit angeregt wird.

Unter anderem aufgrund des im vorhergehenden Absatz beschriebenen Nachteils konnte von Maschine „C“ keine Variante mit in 10 Blöcken modulierten Läufernutpositionen gefunden werden, welche über einen weiten Drehzahlbereich oder gar nur für den Bemessungsbetrieb signifikant leiser erscheint als der Basismotor mit unmodulierten Nutpositionen. 


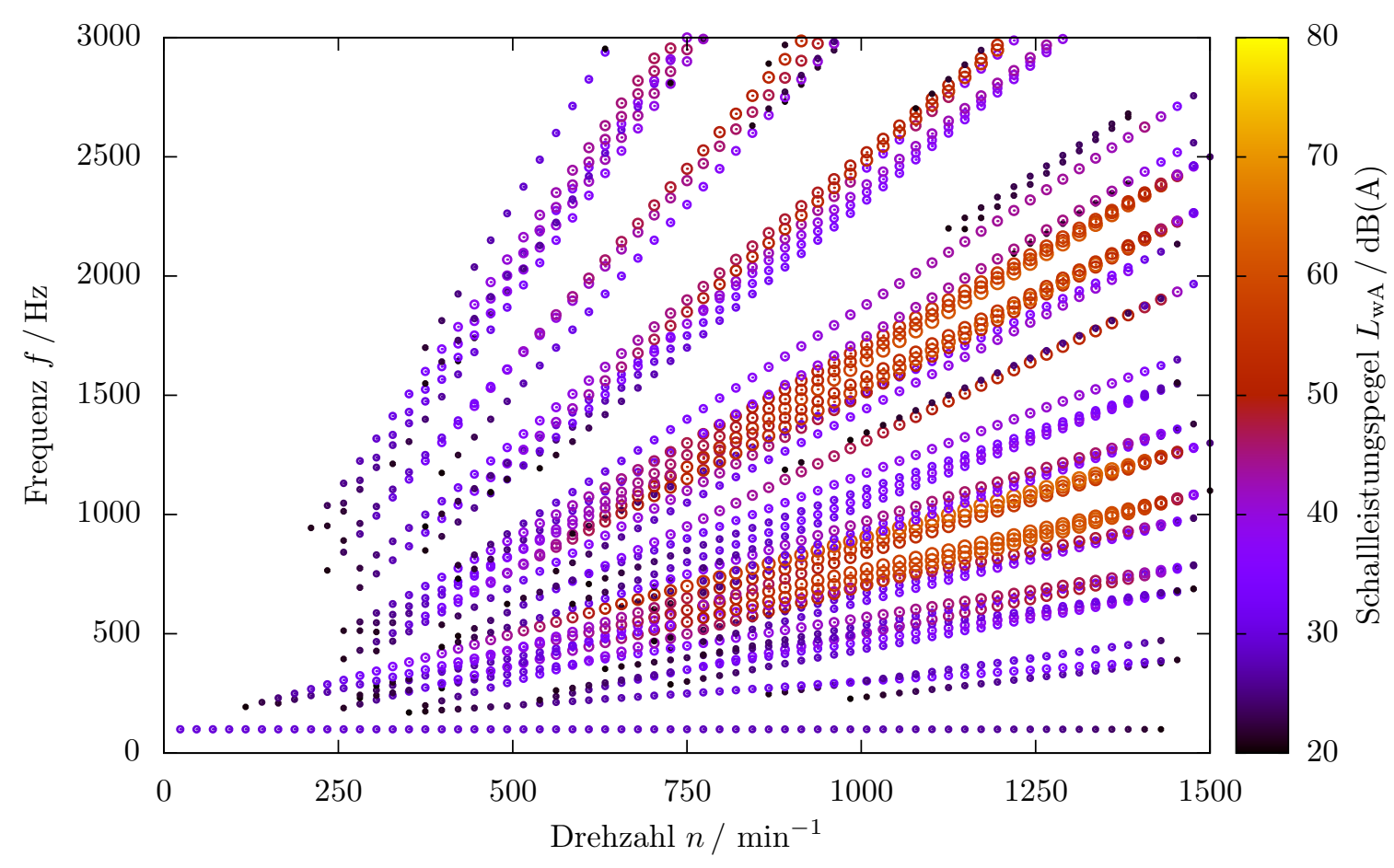

(a) In $b_{2}=4$ Blöcken gemäß Bild $7.5 b$ angeordnete Läufernuten

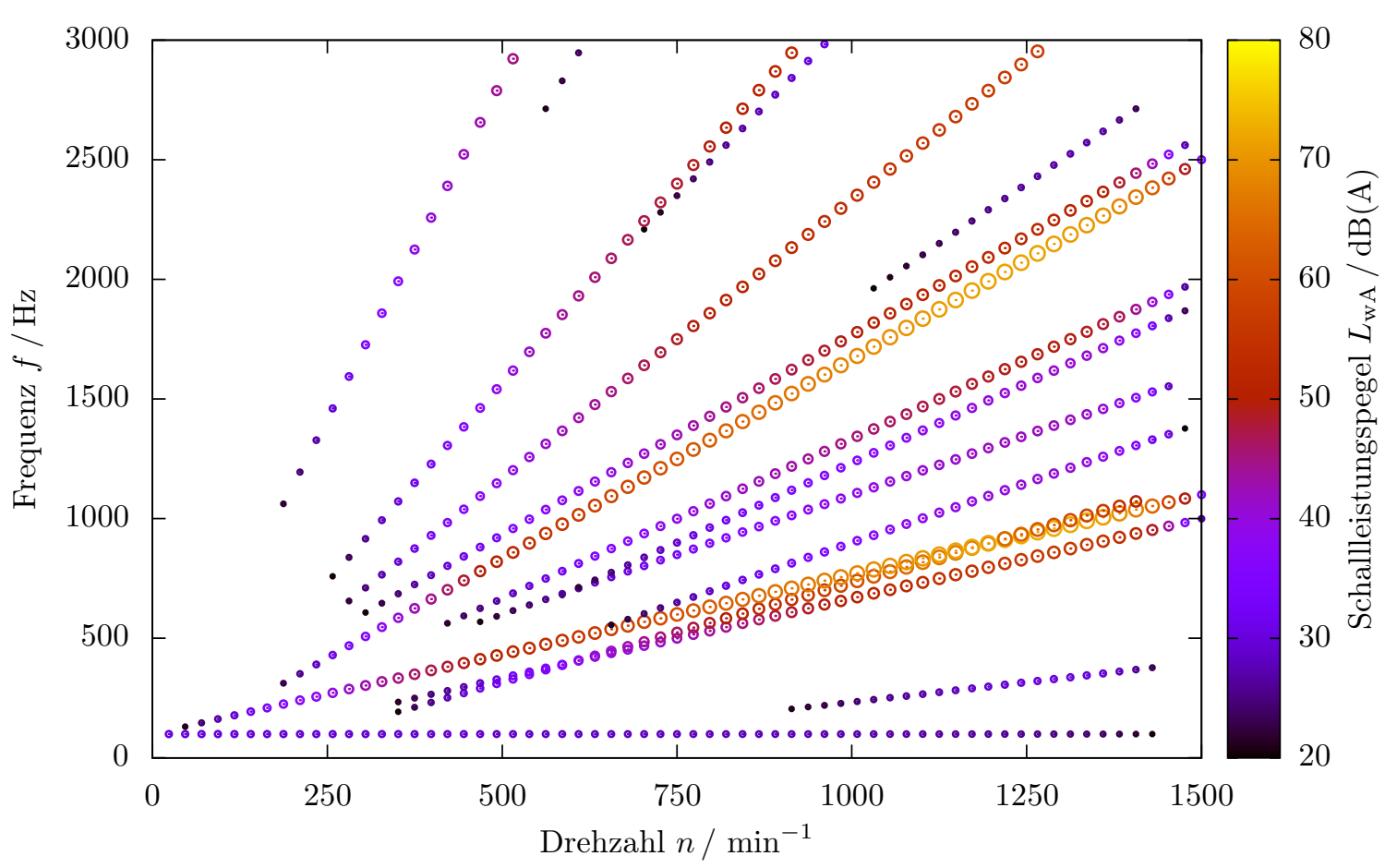

(b) In $b_{2}=10$ Blöcken gemäß Bild $7.5 \mathrm{c}$ angeordnete Läufernuten

Bild 7.7: Schallleistung der Einzeltöne von Maschine „C“ mit $N_{2}=40$-nutigem Läufer bei Betrieb an $U_{1, \text { str }}=398 \mathrm{~V}$ mit $f_{1}=50 \mathrm{~Hz}$ über der Drehzahl und Frequenz 


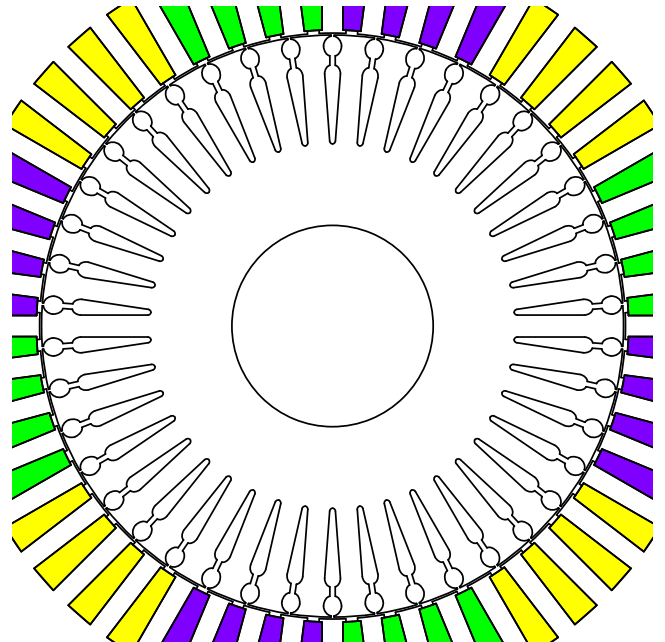

(a) Unmodulierter Läufer

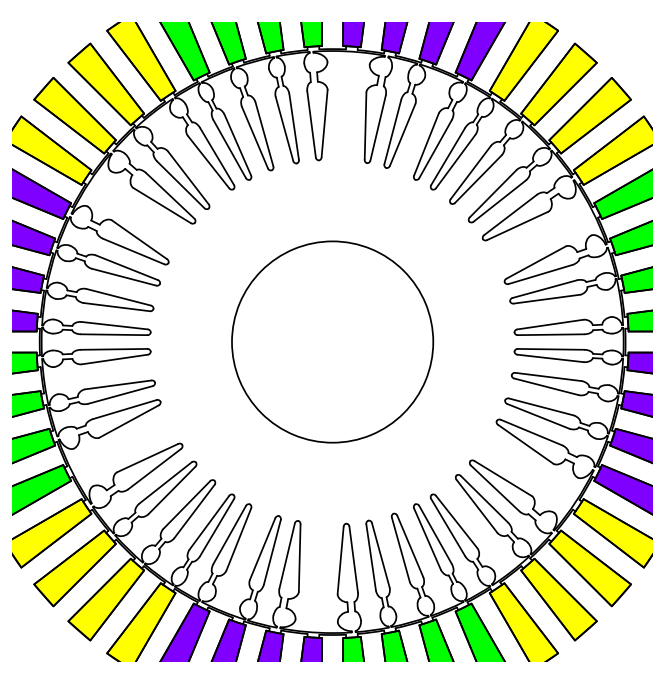

(b) Läufer mit in $b_{2}=6$ gleichen Blöcken modulierten Nutpositionen

Bild 7.8: Blechschnitt von Maschine „C" mit $N_{2}=42$ Läufernuten

Die Geometrie einer Variante mit $b_{2}=10$ Blöcken, deren in Bild $7.4 \mathrm{c}$ dargestelltes Spektrum einen lautesten Einzelton zeigt, welcher um weniger als $1 \mathrm{~dB}(\mathrm{~A})$ unterhalb des lautesten Einzeltones der unmodulierten Variante liegt, ist in Bild $7.5 \mathrm{c} \mathrm{zu}$ sehen. Es ist deutlich zu erkennen, dass die Modulation sehr schwach ausgeprägt ist, da sonst neue laute Einzeltöne entstehen würden. Folglich zeigt sich über der Drehzahl, wie in Bild 7.7b zu sehen ist, ein entgegen der in Bild 7.6 dargestellten Variante nur schwach aufgeweitetes Geräuschspektrum.

\subsubsection{2 $N_{2}=42$ Nuten}

Um ein Beispiel zu zeigen, welches eine deutliche Reduktion der Lautheit durch Modulation der Läufernutpositionen erlaubt, wird eine Variante von Maschine "C" mit $N_{2}=42$ Läufernuten betrachtet, wie diese in Bild [7.8a dargestellt ist.

In dem für diese Variante von Maschine „C“ in Bild [7.9a beispielhaft für den Bemessungspunkt gezeigten Schallleistungsspektrum fallen insbesondere zwei Einzeltöne bei $f=932,5 \mathrm{~Hz}$ und $f=1132,5 \mathrm{~Hz}$ ins Gewicht. Der letztgenannte Spektralanteil entsteht zum Beispiel aus dem Zusammenwirken der ständernutharmonischen Induktionswelle mit $\mu=p-N_{1}=2-48=-46$ und der läufernutharmonischen Induktionswelle mit $\tilde{\nu}=p+N_{2}=2+42=44$, womit sich eine Jochzugwelle der räumlichen Ordnungszahl $\nu=2$ ergibt.

Die $N_{2}=42$ Läufernuten lassen sich bei Berücksichtigung einer Symmetrie zur Vermeidung von Rüttelkräften in 2, 6 oder 14 gleichen Blöcke mit je 21, 7 oder 3 Nuten anordnen.

Für $b_{2}=6$ Blöcke ist eine optimierte Geometrie in Bild $7.8 \mathrm{~b}$ zu sehen, die, wie in Bild [7.9] und Bild [7.10 zu erkennen, zu einer signifikanten Amplitudenreduktion der lautesten Einzeltöne führt.

Das hier vorgestellte Beispiel zeigt deutlich, dass die Lautheit einer Maschine durch Variation der Läufernutpositionen verringert werden kann. Gleichzeitig zeigt ein Vergleich 


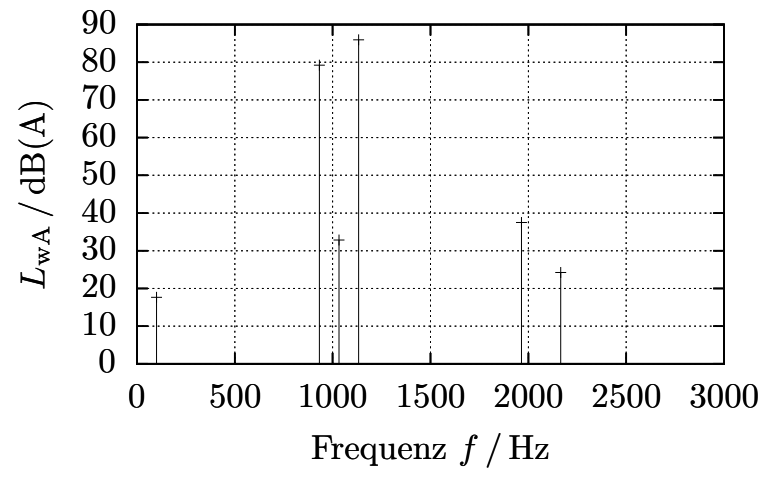

(a) Unmodulierter Läufer

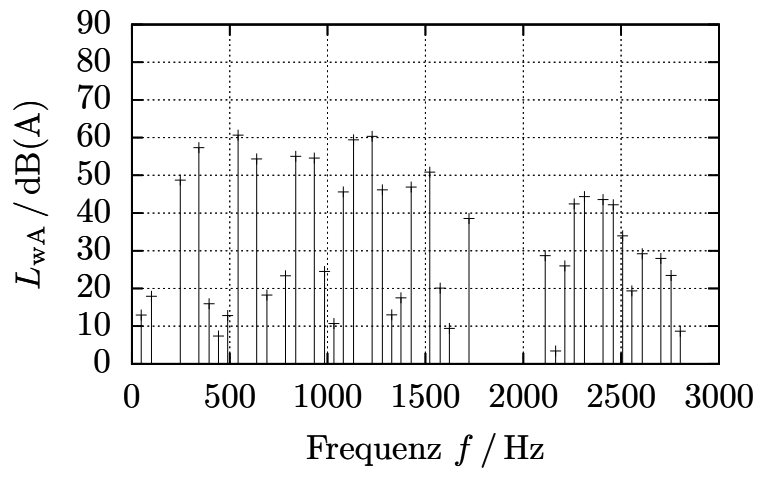

(b) Modulierter Läufer gemäß Bild 7.8b

Bild 7.9: A-bewerteter Schallleistungspegel $L_{\mathrm{wA}}(f)$ von Maschine „C" mit $N_{2}=42$-nutigem Läufer bei der Drehzahl $n=1475 \mathrm{~min}^{-1}$ und dem Strangstromeffektivwert $I_{1}=14,12 \mathrm{~A}$

der Geräuschspektren der gleichmäßig genuteten Variante mit $N_{2}=40$ Läufernuten in Bild [7.6] und der optimierten Variante mit $N_{2}=42$ Läufernuten in Bild 7.10b, dass eine geschickte Wahl der Läufernutzahl einen deutlich größeren Effekt, als die Optimierung des Geräuschs durch Variation der Nutpositionen haben kann.

Robustheit Zur Abschätzung der Robustheit der Modulation der Läufernutpositionen gegenüber Fertigungsungenauigkeiten, ist für Maschine „C" mit $N_{2}=42$-nutigem Läufer gemäß Bild 7.8b in Bild 7.11 die Schallleistung der Einzeltöne über der Abweichung der Nutpositionen aufgetragen. Dabei handelt es sich bei der dargestellten Abweichung um eine Verschiebung aller Nuten in jeweils beliebige, für alle berechneten Punkte aber gleiche Richtung. Die Nutteilung des gleichmäßig aufgebauten Läufers beträgt dabei in etwa $12 \mathrm{~mm}$.

Für das gezeigte Beispiel lässt sich insbesondere in Bild 7.11b bereits bei Positionsabweichungen von wenigen hundertstel Millimetern eine Aufweitung des Geräuschspektrums erkennen. Zudem zeigt Bild 7.11a ein deutliches Aufklingen der lautesten Einzeltöne bei Positionsabweichungen ab wenigen Zehntelmillimetern.

Es lässt sich damit schlussfolgern, dass die Variation der Läufernutpositionen zur Reduktion der Lautheit von elektrischen Maschinen eine hohe, üblicherweise aber erreichbare Fertigungsgenauigkeit voraussetzt.

\subsubsection{3 $N_{2}=48$ Nuten}

Bei der Betrachtung einer Variante von Maschine "C" mit $N_{2}=N_{1}=48$ Läufernuten soll der Fokus nicht auf das Geräusch, sondern das Drehmoment gerichtet werden, dessen zeitinvariante Anteile signifikant von einer Läufernutmodulation abhängig sind.

Wird bei einer gleichmäßig genuteten ungeschrägten Induktionsmaschine die Ständernutzahl gleich der Läufernutzahl gewählt, wie dies in Bild [7.12a zu sehen ist, so ergeben sich im Stillstand der Maschine, also bei $\Omega=0$, hohe synchrone Drehmomente, welche das Anlaufen der Maschine verhindern. Im vorliegenden Beispiel, für das eine gegenüber dem Original von Maschine „C“ verschiedene Läufernutgeometrie gewählt wurde, bedeutet 


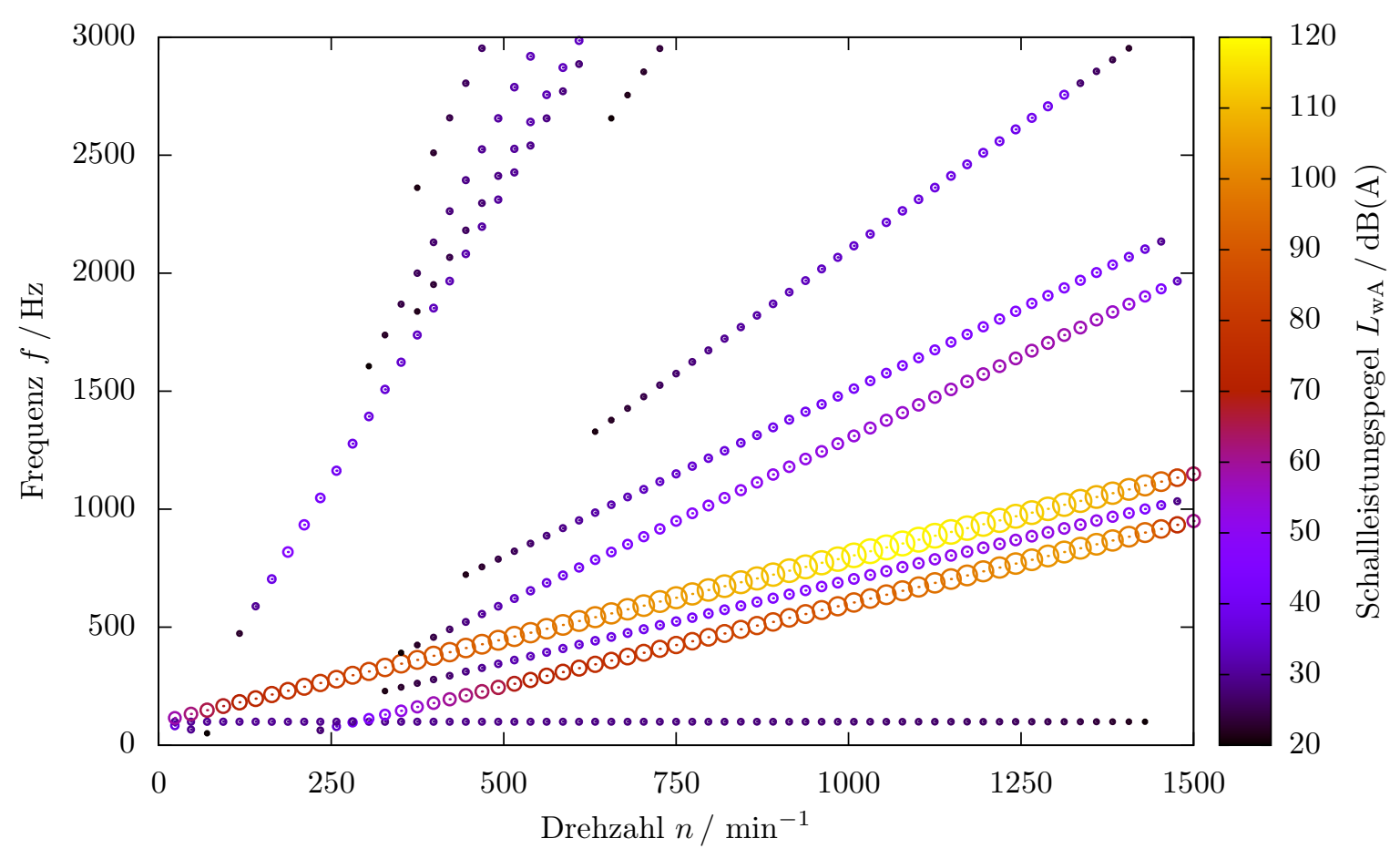

(a) Gleichmäßig genuteter Läufer

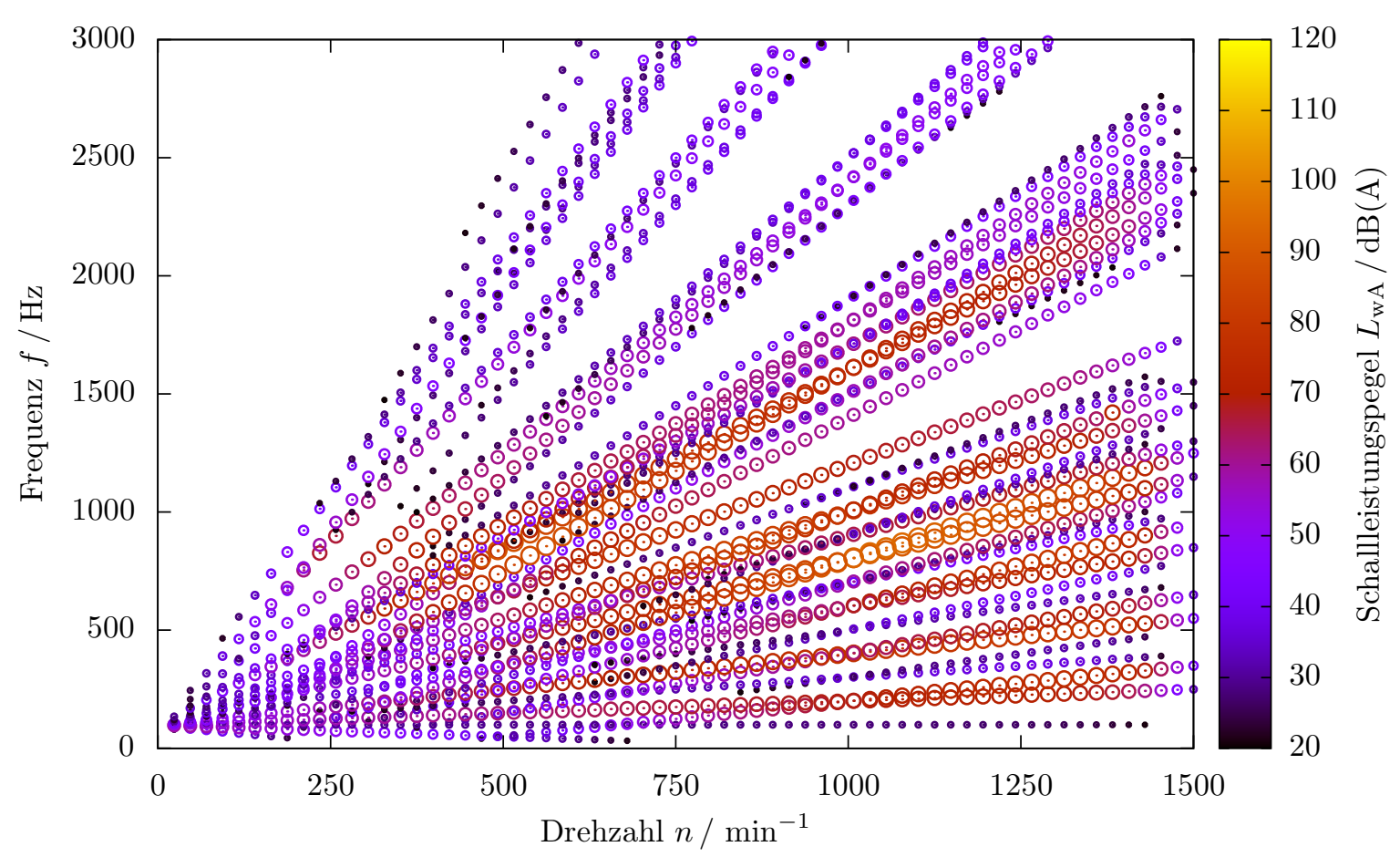

(b) In 6 Blöcken gemäß Bild $7.8 \mathrm{~b}$ angeordnete Läufernuten

Bild 7.10: Schallleistung der Einzeltöne von Maschine „C“ mit $N_{2}=42$-nutigem Läufer bei Betrieb an $U_{1, \text { str }}=398 \mathrm{~V}$ mit $f_{1}=50 \mathrm{~Hz}$ über der Drehzahl und Frequenz 


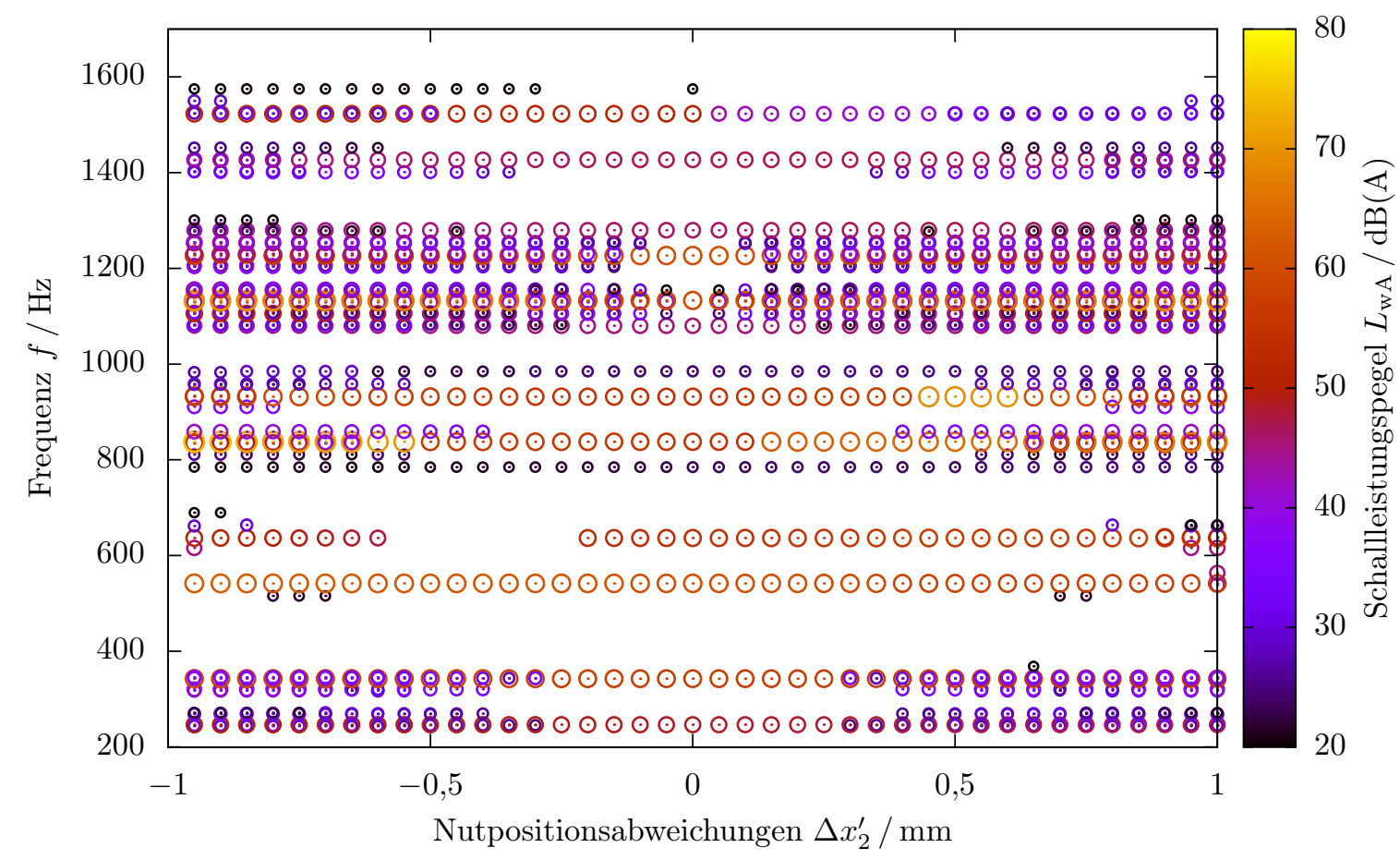

(a) Nutpositionsabweichungen von $\pm 1 \mathrm{~mm}$

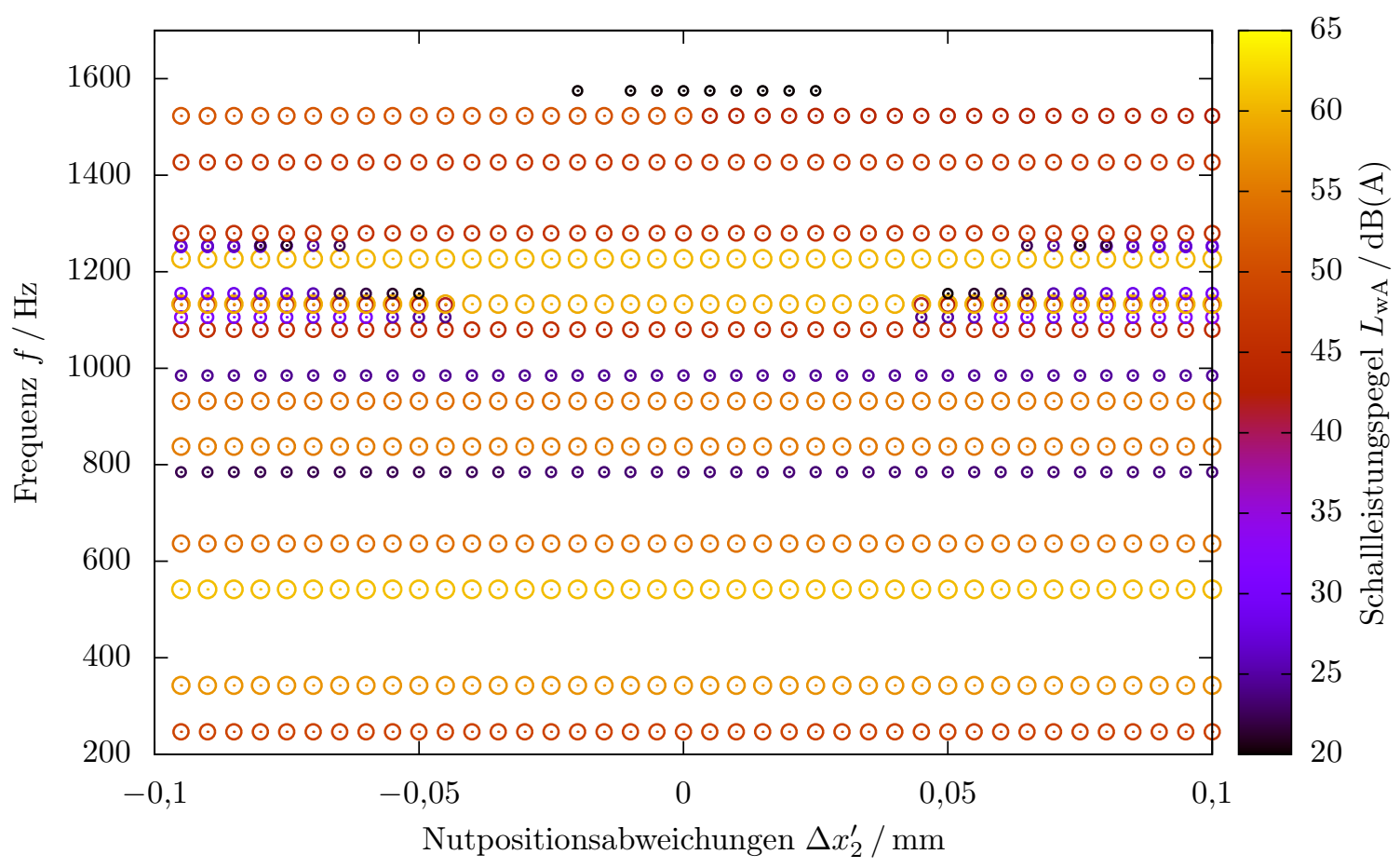

(b) Nutpositionsabweichungen von $\pm 0,1 \mathrm{~mm}$

Bild 7.11: Abhängigkeit der Schallleistung von Maschine „C“ mit $N_{2}=42$ in 6 Blöcken gemäß Bild 7.8b angeordneten Nuten von einer Verschiebung der Nuten in beliebiger, für alle Punkte aber gleicher Richtung je Nut 


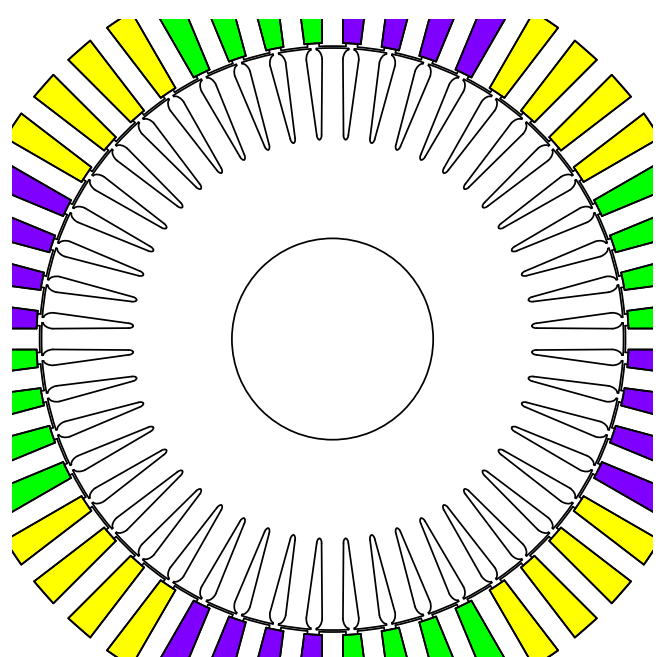

(a) $N_{2}=48$ gleichmäßig angeordnete Läufernuten

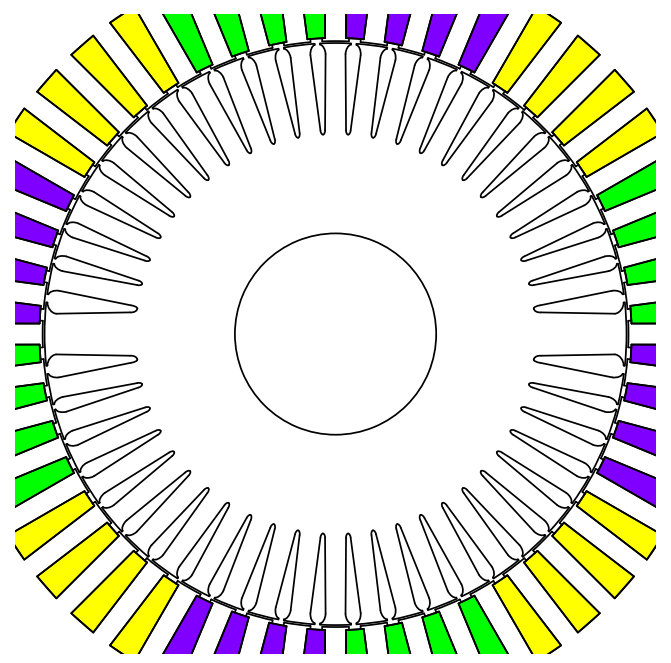

(c) $N_{2}=48$ Läufernuten an den Positionen der $\tilde{N}_{2}=50$ Läufernuten gemäß Bild 7.12 b

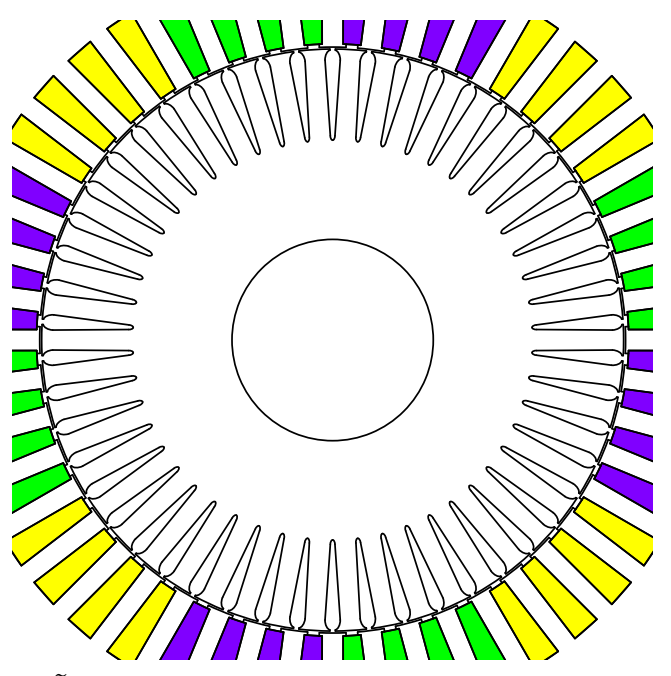

(b) $\tilde{N}_{2}=50$ gleichmäßig angeordnete Läufernuten

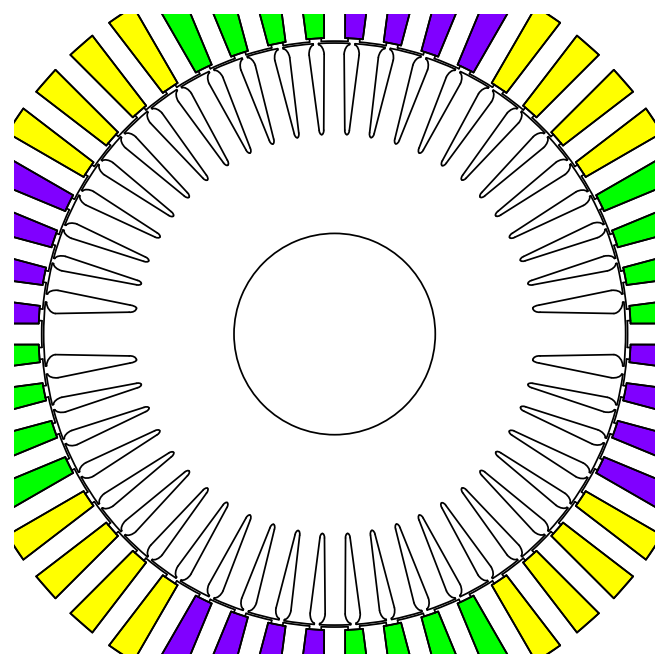

(d) $N_{2}=48$ numerisch optimiert angeordnete Läufernuten

Bild 7.12: Blechschnitt von Maschine „C“ mit $N_{2}=48$ Läufernuten

dies, dass die Maschine bei Wahl eines Läufers mit $N_{2}=48$ Nuten und nicht modulierten Läufernutpositionen nicht anlaufen würde.

Wenn hingegen gemäß Bild 7.12b ein Läufer mit $\tilde{N}_{2}=50$ Nuten gewählt würde, so ergäbe sich das in Bild 7.13b dargestellte durchweg positive quasistationäre Drehmoment über dem Drehwinkel des Läufers $\rho$, sodass die Maschine sicher anlaufen würde.

Zur praktischen Berechnung, bei welchen Geometrien eine Maschine anläuft, kann direkt die in den vorherigen Kapiteln aufgebaute Theorie verwendet werden. Hierbei soll zunächst jedoch nur der Anlauf und nicht das Erreichen des Kipppunkts des von der Hauptwelle generierten Drehmoments betrachtet werden.

Bei Annahme einer sehr kleinen Drehzahl mit $n \ll f_{1}$ teilen sich die Komponenten im Drehmoment in Anteile nahe der doppelten Ständerfrequenz $f=2 \cdot f_{1}$ und nahe $f=0$ auf. Dabei bilden die Anteile nahe $f=0$ das winkelabhängige quasistationäre Drehmoment im Stillstand der Maschine ab. Ist dieses unabhängig von der Zeit $t$ beziehungsweise dem 
mechanischen Winkel des Läufers $\rho$ größer als die phasenrichtig gebildete Summe aller zeitvarianten Drehmomentanteile niedriger Frequenz, so kann von einem sicheren Anlauf der Maschine ausgegangen werden.

Vereinfacht kann der Anlauf der Maschine durch eine Addition der Beträge der im letzten Absatz genannten Drehmomentanteile überprüft werden. Ist die Summe der Beträge kleiner als der Mittelwert des Drehmoments, so impliziert dies, dass die Forderung des vorhergehendes Absatzes erfüllt ist. Es handelt sich hierbei folglich um eine hinreichende und im vorhergehenden Absatz um eine notwendige Bedingung.

Um nun mit $N_{2}=48$ Läufernuten den Anlauf der Maschine zu gewährleisten, können mit den 48 Nuten die oben genannten 50 Nuten angenähert werden, indem die 48 Nuten zum Beispiel im Abstand von $\frac{360^{\circ}}{50}=7,2^{\circ}$ angeordnet werden, sodass quasi zwei der $\tilde{N}_{2}=50$ Nuten entfallen. Eine vergleichbare Anordnung, bei der die Läufernuten jedoch im Abstand von

$$
\frac{360^{\circ}}{N_{2}}-\frac{2 \cdot 360^{\circ}}{N_{2}^{2}}=\frac{46 \cdot 360^{\circ}}{48^{2}}=7,1875^{\circ}
$$

angeordnet sind, sodass sich gerade genau 24 gleich verteilte Differenzwinkel zwischen der $n$-ten Läufer- und der $n$-ten Ständernut ergeben, ist in Bild $7.12 \mathrm{c}$ zu sehen. Das entstehende und in Bild 7.13c gezeigte quasistationäre Drehmoment bei niedriger Drehzahl ist dabei durchweg positiv, sodass die Maschine im unbelasteten Zustand voraussichtlich anlaufen würde.

Eine deutliche Erhöhung des minimalen quasistationären Drehmoments über dem Drehwinkel des Läufers $\rho$ lässt sich durch eine numerische Optimierung der Geometrie gemäß Bild $7.12 \mathrm{c}$ unter ausschließlicher Bewertung genau dieses Drehmomentminimums erreichen. Beispielhaft ist hierzu in Bild 7.12d eine Geometrie sowie in Bild 7.13d das zugehörige Drehmoment dargestellt.

Dieses einfache Beispiel zeigt, dass bei Variation der Läufernutpositionen, viele gängige Entwurfsregeln für Induktionsmaschinen, wie zum Beispiel die Forderung nach $N_{1} \neq N_{2}$ zur Sicherstellung des Anlaufs, ihre Gültigkeit verlieren.

\subsection{Variation der Ständergeometrie}

Zur Variation der Ständergeometrie wird das in Kapitel [6] entwickelte Verfahren herangezogen.

\subsubsection{Maschine „A“}

Bezüglich der Variation der Ständergeometrie stellt Maschine „A“ eine Besonderheit dar. Aufgrund der Wicklung mit $q_{1}=2$ Ständernuten je Pol und Strang besteht bereits Phasenrichtigkeit der Zahnflüsse. Damit beschränkt sich bei dieser Maschine die in Kapitel [6 vorgeschlagene Variation der Ständergeometrie auf eine Anpassung der Luftspaltweite. Es ergibt sich eine Geometrie entsprechend Bild 7.14b beziehungsweise Bild $7.14 \mathrm{c}$

Durch Sehnung mit der in Bild 7.14a dargestellten Spulenweite von 5 Nuten wird bei der Lochzahl $q_{1}=2$ von Maschine „A“ ebenfalls Gleichheit der Induktionsamplituden im 


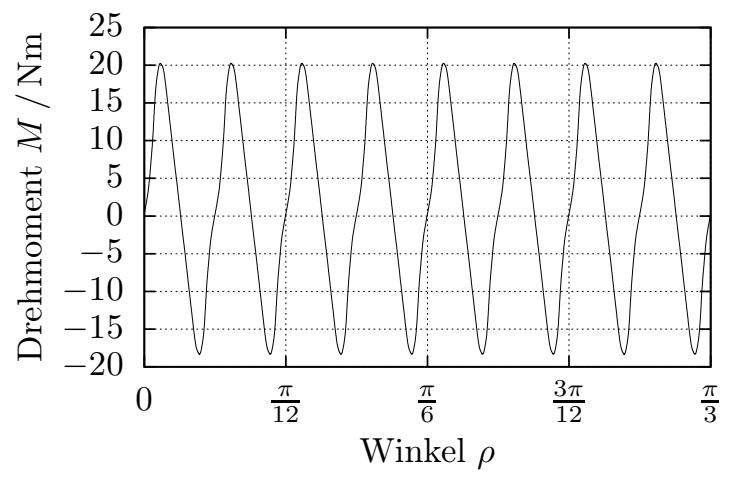

(a) Läufer mit $N_{2}=48$ Nuten

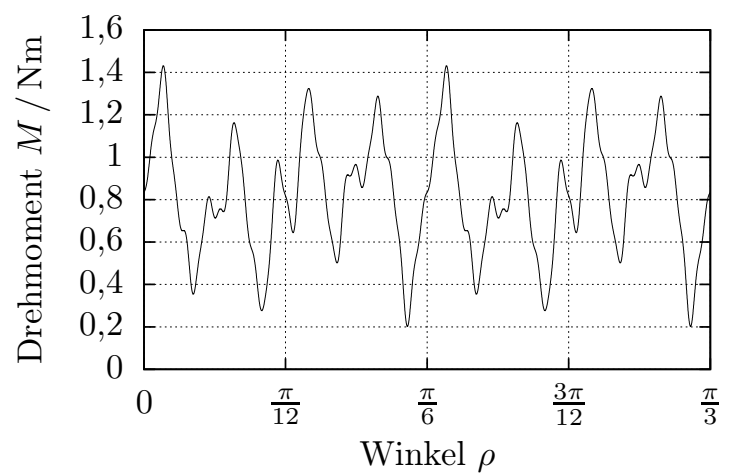

(c) Läufer mit $N_{2}=48$ Nuten bei Annäherung an 50 Nuten

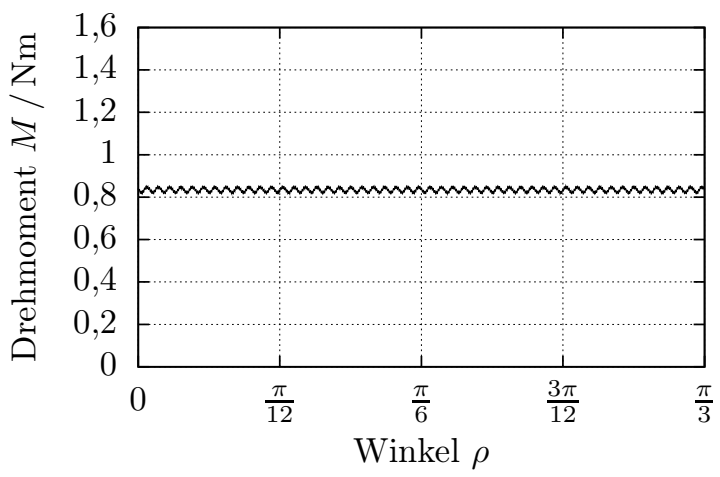

(b) Läufer mit $N_{2}=50$ Nuten

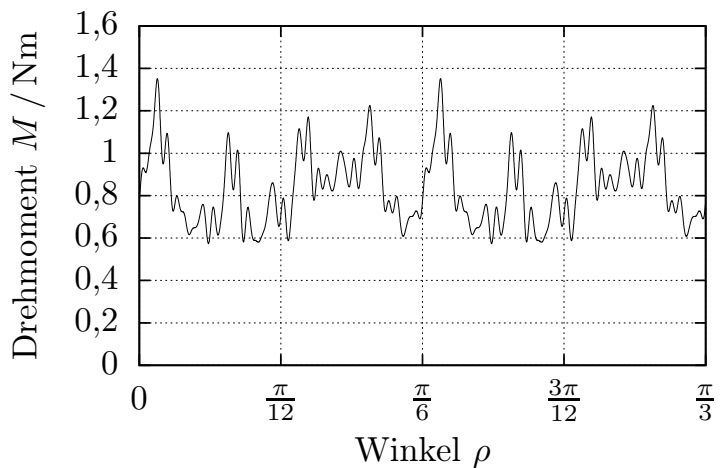

(d) Läufer mit $N_{2}=48$ Nuten bei optimierter Annäherung an 50 Nuten

Bild 7.13: Quasistationäres Drehmoment von Maschine „C“ mit ungeschrägten Läufern gemäß Bild 7.12 in Abhängigkeit vom Drehwinkel des Läufers $\rho$ 


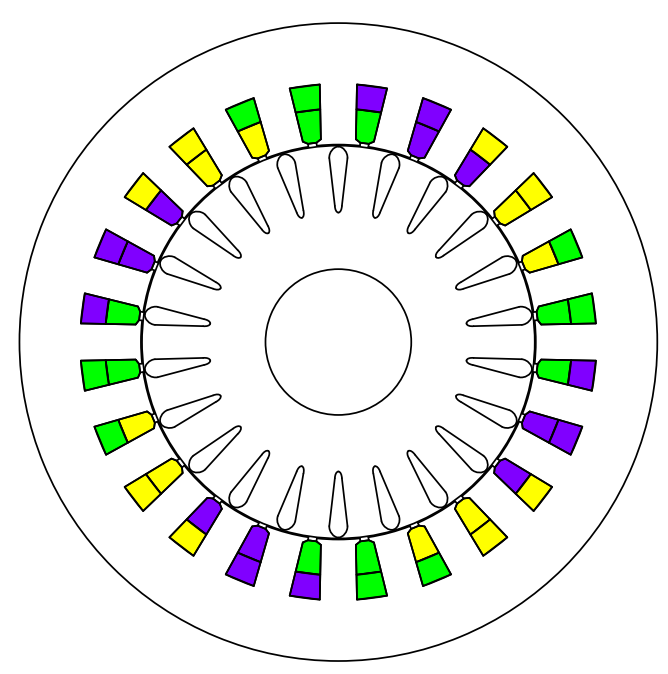

(a) Gesehnte Zweischichtwicklung

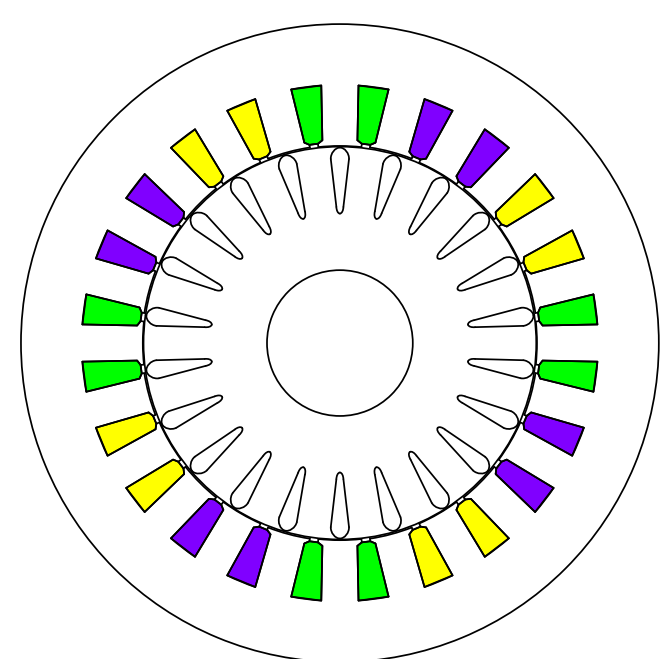

(b) Einschichtwicklung; Luftspaltweitenmodulation fünffach überhöht dargestellt

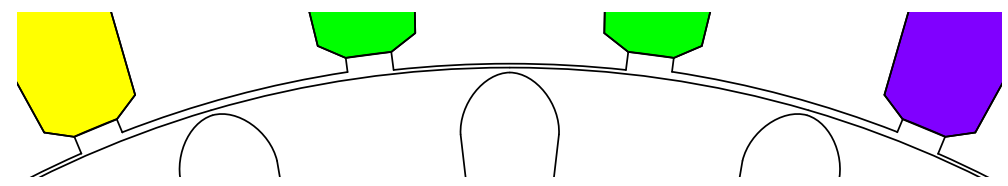

(c) Einschichtwicklung; Luftspaltweitenmodulation fünffach überhöht dargestellt - Ausschnittvergrößerung

Bild 7.14: Geometrie von Maschine „A“

Bereich aller Ständerzähne bei Phasenrichtigkeit erreicht. Dies lässt sich leicht mit Hilfe von (6.7) auf Seite 71] verifizieren.

Wie in Tabelle $7.7 \mathrm{zu}$ erkennen, ergeben sich bei gleichem Ständerstrom und gleicher Windungszahl für alle drei Varianten, nämlich die ungesehnte sowie gesehnte Variante mit herkömmlichem Blechschnitt und die ungesehnte Variante bei Anpassung der Luftspaltweite, verschiedene Induktionshauptwellenamplituden. Die Tatsache, dass die Variation der Luftspaltweite zur geringsten Amplitude führt, ist in der Wahl der Luftspaltweite $\tilde{\delta}$ begründet, wie sie in (6.13) verwendet wird. Im gezeigten Beispiel wurde $\tilde{\delta}$ derart gewählt, dass die minimale geometrische Luftspaltweite für alle Varianten gleich ist. Ist eine Verringerung der minimalen mechanischen Luftspaltweite vertretbar, so lässt sich die Induktionshauptwellenamplitude bei Luftspaltweitenmodulation zum Beispiel auch auf das Niveau der gesehnten Variante anheben. Damit ließen sich sowohl für den gesehnten Fall als auch für den Fall mit Variation der Luftspaltweite annähernd gleiche Spektren erreichen, wie dies in Bild 7.15 zu erkennen ist. Die Spektren besitzten dabei nahezu ausschließlich nutharmonische Induktionswellen, also die räumlichen Ordnungszahlen

$$
\nu=p+k \cdot N_{1}=2+k \cdot 24 \text { mit } k \in \mathbb{Z} .
$$

Es lässt sich aus dieser Gegenüberstellung folgern, dass das Verfahren der Variation der Luftspaltweite gegenüber der Sehnung für $q_{1}=2$ einen vergleichbaren Einfluss auf die ständerseitig erregte Luftspaltinduktion erlaubt. Dies gilt unter der Einschränkung, dass die minimale geometrische Luftspaltweite verringert werden kann. Der Vorteil der Variation der Luftspaltweite gegenüber der Sehnung ist dabei, dass auf die Ausführung einer 
Tabelle 7.7: Amplituden der ständerseitig erregten Induktionshauptwellen von Maschine „A“ bei gleichem Ständerstrom und gleichen Windungszahlen

\begin{tabular}{lc}
\hline Variante & $\hat{B}_{\mathrm{p}} /[\mathrm{T}]$ \\
\hline ungesehnt & 1,091 \\
gesehnt & 1,054 \\
Variation der Luftspaltweite & 1,015 \\
\hline
\end{tabular}

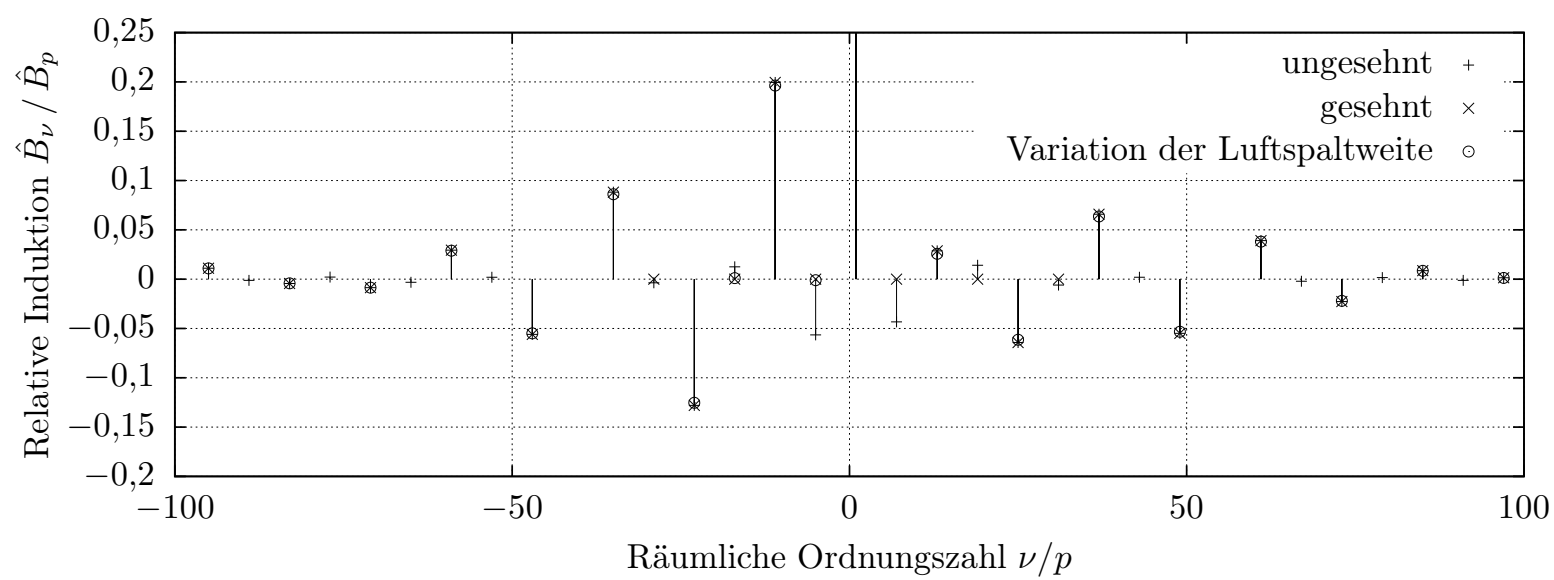

Bild 7.15: Spektren der ständerseitig erregten Luftspaltinduktion von Maschine „A“ bezogen auf die jeweilige Luftspaltinduktionshauptwellenamplitude $\hat{B}_{p}$; Einflüsse der Läufernutung vernachlässigt

Zweischichtwicklung verzichtet werden kann. Es muss hierfür jedoch ein spezieller Blechschnitt mit möglichen Folgekosten für zum Beispiel ein Stanzwerkzeug erstellt werden.

\subsubsection{Maschine „C“}

Aufgrund eines signifikanten Einflusses der Breite der Ständernutschlitze werden nachfolgend sowohl der originale Ständer als auch ein modifizierter Ständer mit schmaleren Nutschlitzen betrachtet.

\subsubsection{Ständer mit schmalen Nutschlitzen}

Um den Einfluss der Ständernutung gering zu halten, wird zunächst eine Variante von Maschine „C" betrachtet, die, wie in Bild 7.16a ersichtlich, gegenüber dem Original schmalere Nutschlitze im Ständer besitzt. Um die Wicklung trotz der schmaleren Nutschlitze einbringen zu können, müssen dabei dünnere Teilleiter verwendet werden.

Die ständerseitig erregte Luftspaltinduktion dieser modifizierten Maschine „C" ist für den unvariierten Fall in Bild 7.17a zum Zeitpunkt $t=0$ über der Winkelkoordinate $x_{1}$ aufgetragen. Zusätzlich enthält die Bild die um den Kehrwert des Carter'schen Faktors $1 / k_{\mathrm{c}}$ vergrößerte Hauptwelle der Luftspaltinduktion desselben Zeitpunkts. 


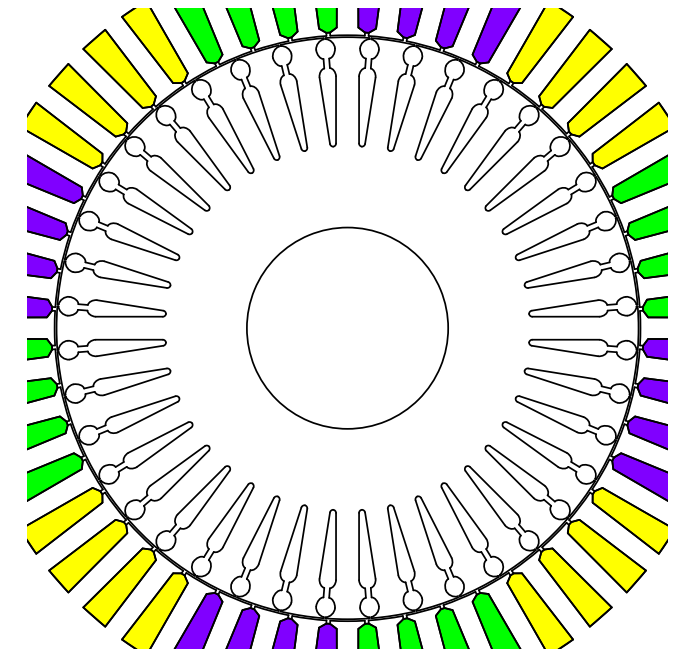

(a) Unmodulierter Ständer und Läufer

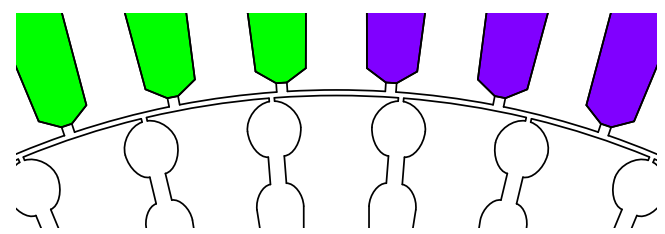

(c) Unmodulierter Ständer und Läufer - Ausschnittvergrößerung

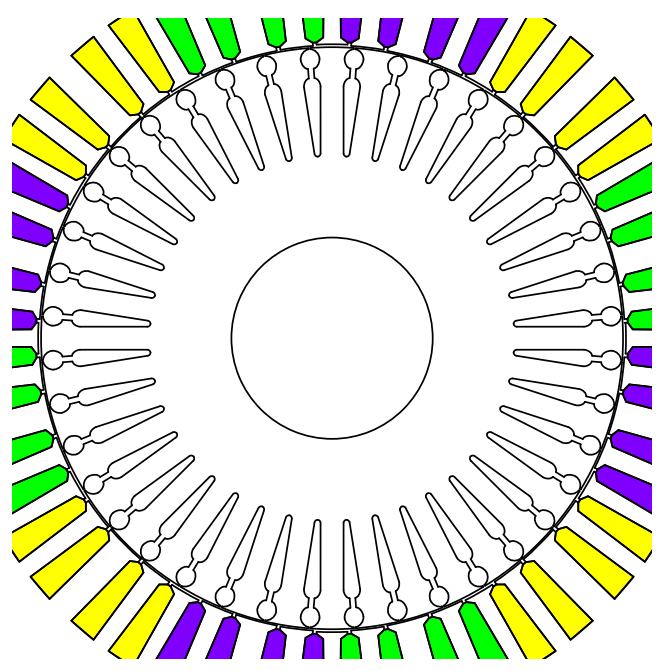

(b) Ständer mit Phasenwinkelkorrektur und fünffach überhöht dargestellter Luftpaltweitenmodulation

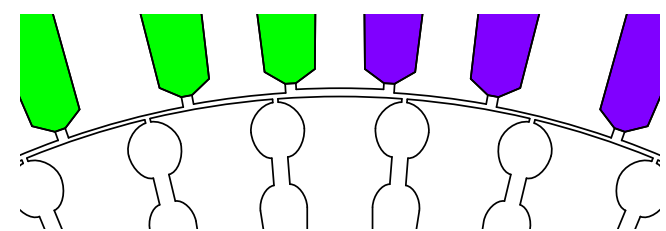

(d) Ständer mit Phasenwinkelkorrektur und fünffach überhöht dargestellter Luftpaltweitenmodulation - Ausschnittvergrößerung

Bild 7.16: Blechschnitt von Maschine „C“ mit schmaleren Ständernutschlitzen 


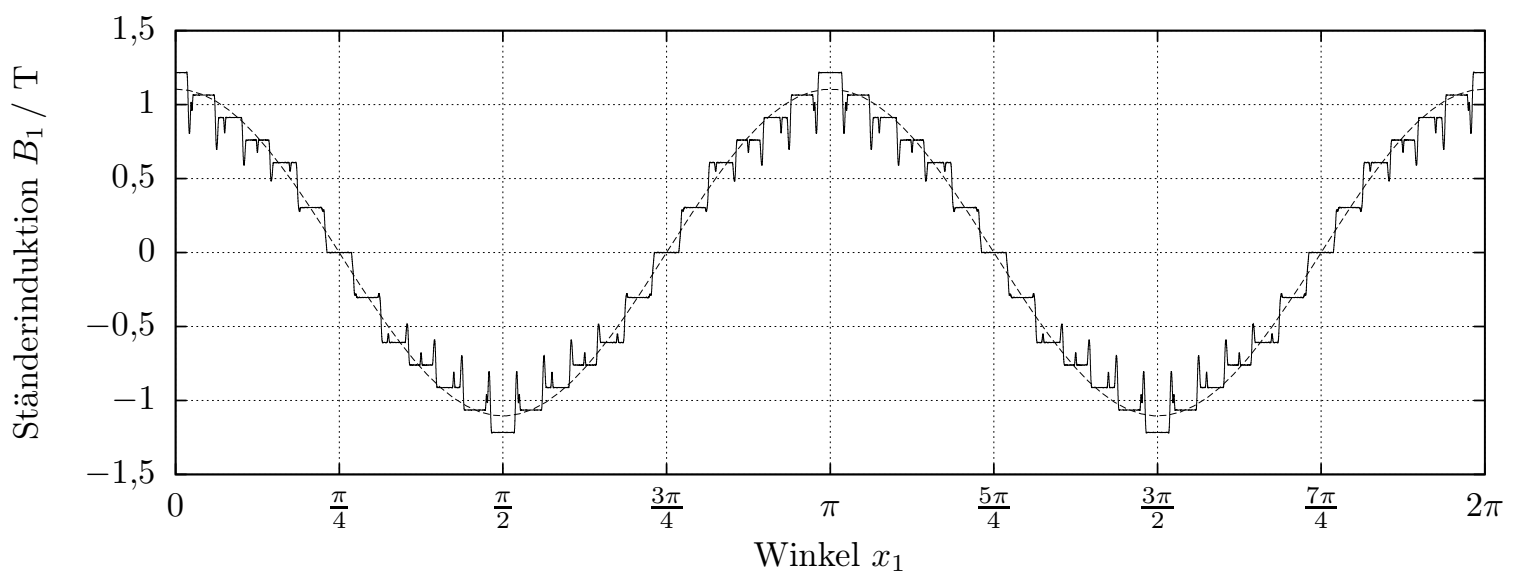

(a) Induktionsverlauf $B_{1}\left(x_{1}, t=0\right)$ bei gleichmäßiger Ständergeometrie

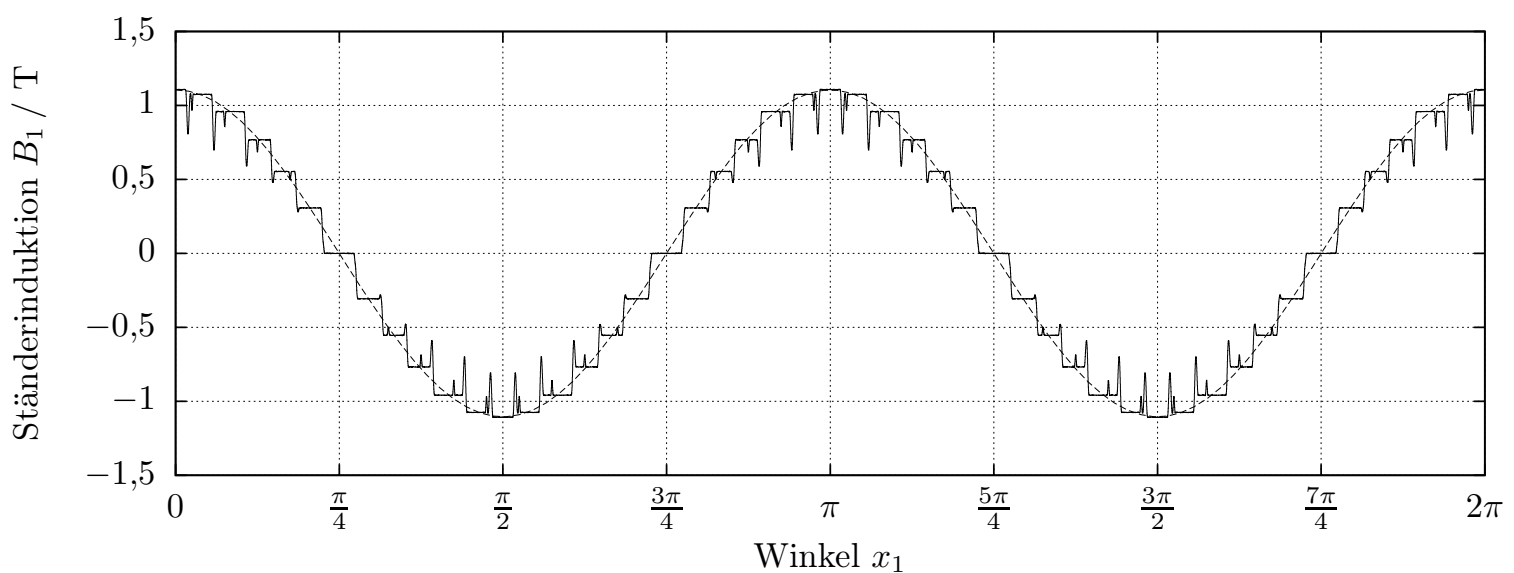

(b) Induktionsverlauf $B_{1}\left(x_{1}, t=0\right)$ bei Luftspaltweitenanpassung und Phasenwinkelkorrektur

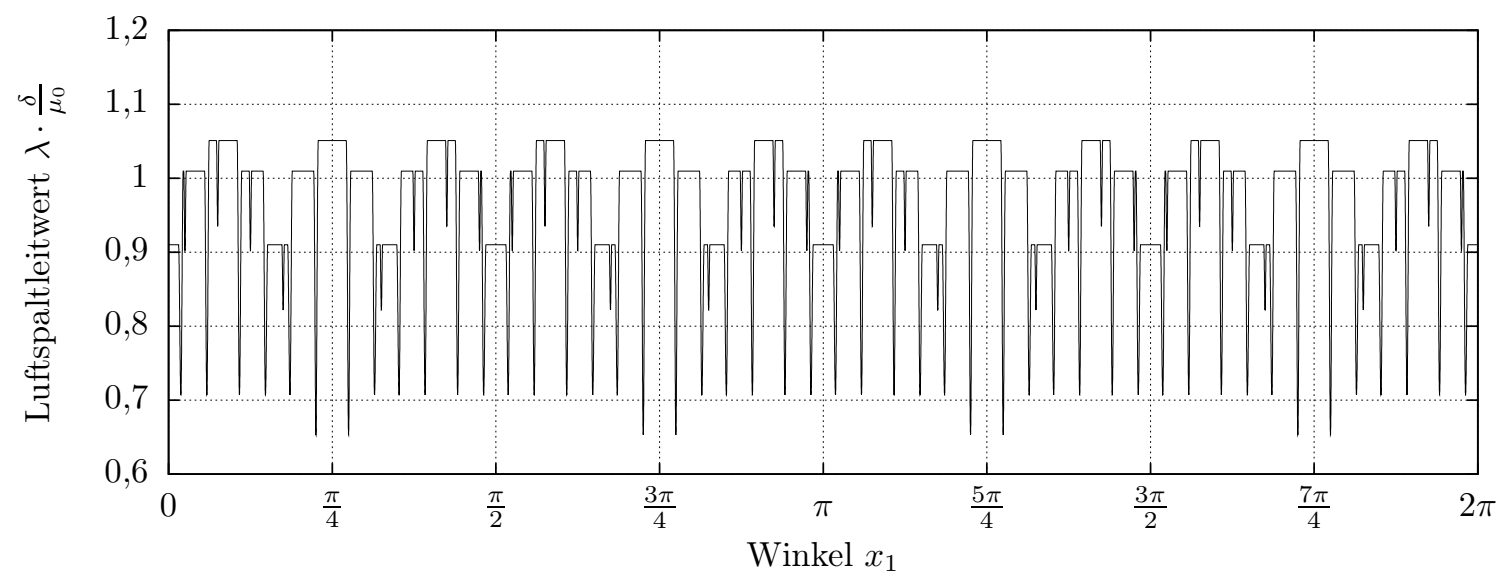

(c) Normierter Luftspaltleitwert $\frac{\delta}{\mu_{0}} \cdot \lambda\left(x_{1}, t=0\right)$

Bild 7.17: Einfluss der Nutpositionen und Luftspaltweiten auf die ständerseitig erregte Induktion von Maschine „C“" mit schmalen Ständernutschlitzen. - Neben dem resultierenden Induktionsverlauf, in dem der Einfluss der Läufernutung erkennbar ist, sind jeweils die um den Kehrwert des Carter'schen Faktor $1 / k_{\mathrm{c}}$ vergrößerten Induktionshauptwellen dargestellt. 


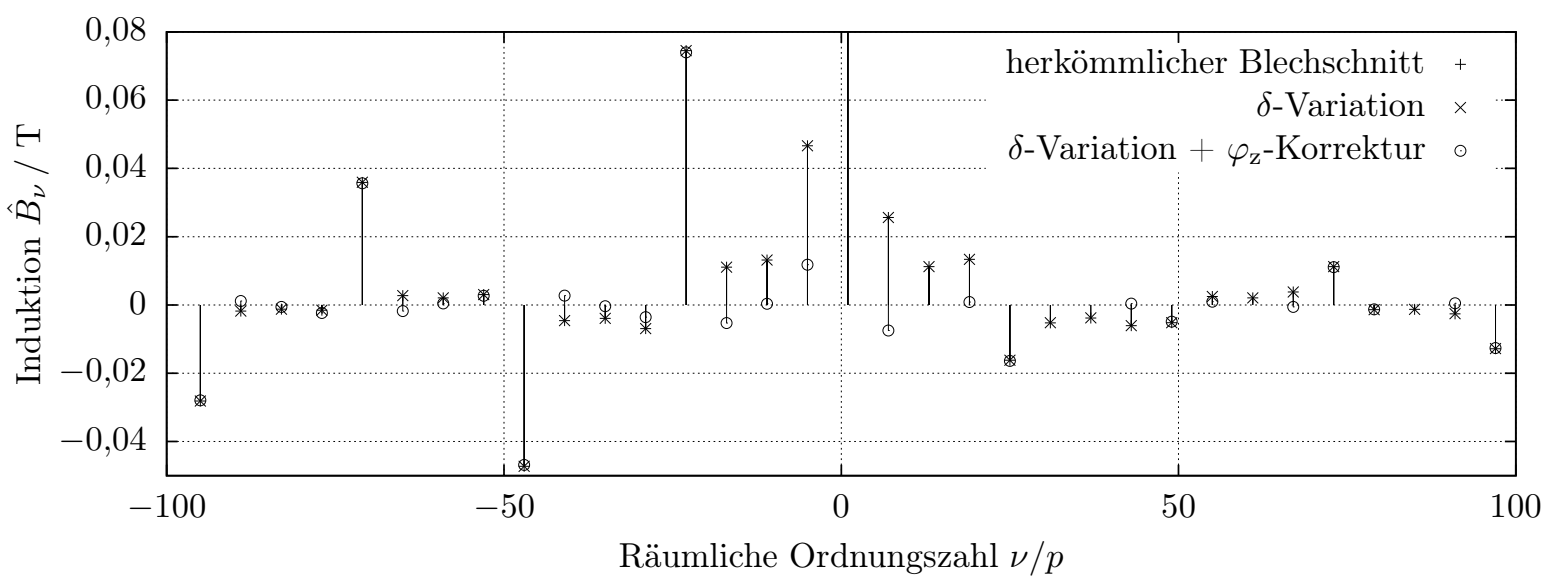

Bild 7.18: Spektrum der ständerseitig erregten Luftspaltinduktion von Maschine „C“ mit schmalen Ständernutschlitzen und herkömmlichem Blechschnitt, Variation der Luftspaltweite $(\delta$-Variation) sowie Variation der Luftspaltweite und gleichzeitiger Phasenwinkelkorrektur $(\delta$ Variation $+\varphi_{\mathrm{z}}$-Korrektur); die Luftspaltweite $\tilde{\delta}$ gemäß (6.13) ist jeweils derart gewählt, dass sich in allen Varianten die gleiche Induktionshauptwellenamplitude ergibt.

Mit den Ausführungen in Kapitel [6] lässt sich erwarten, dass die Variation der Luftspaltweiten eine deutliche Reduzierung aller nichtnutharmonischen Induktionswellen mit sich bringt.

Wie im Vergleich der Spektren der Variante mit herkömmlichem Blechschnitt und der Variante mit Variation der Luftspaltweite in Bild 7.18 ersichtlich, fällt diese Reduzierung jedoch nur schwach aus. Erst unter Einbeziehung der in Abschnitt 6.4 vorgestellten Phasenwinkelkorrektur, womit sich der in Bild 7.17b dargestellte Induktionsverlauf ergibt, lässt sich in dem zugehörigen und in Bild 7.18 dargestellten Spektrum eine deutliche Reduzierung der nichtnutharmonischen Induktionswellen erkennen.

Der Vergleich von Bild 7.17 a und Bild 7.17b zeigt weiterhin, dass sich mit der Anwendung von Luftspaltweitenmodulation und Phasenwinkelkorrektur im Gegensatz zur unvariierten Geometrie weitgehend mittig auf der Hauptwelle liegende Stufen der Luftspaltinduktion ergeben. Es wird damit deutlich, dass das Ziel eines möglichst oberwellenarmen Luftspaltinduktionsfelds weitgehend erreicht werden kann.

Es sei angemerkt, dass die in (6.13) eingeführte Luftspaltweite $\tilde{\delta}$ in den in Bild [7.17] und Bild 7.18 gezeigten Beispielen gerade derart gewählt wurde, dass die Mittelwerte der Luftspaltleitwerte bei unmoduliertem und moduliertem Ständer zur besseren Vergleichbarkeit der Induktionen identisch sind.

Alternativ hätte $\tilde{\delta}$ auch, wie in dem in Abschnitt [7.4.1 vorgestellten Beispiel von Maschine „A“, derart gewählt werden können, dass die minimalen Luftspaltweiten von modulierter und unmodulierter Maschine gerade identisch sind.

Der Nachteil der erstgenannten Variante ist die geringere minimale geometrische Luftspaltweite und der Nachteil der zweitgenannten Variante die größere effektive Luftspaltweite, sodass sich bei gleicher Ständerfelderregung eine geringere Induktion ergäbe. Um diese beiden Nachteile gering zu halten, wurde bei der Gestaltung der in Bild 7.16b dargestellten Geometrie der in Abschnitt 6.4 genannte Freiheitsgrad, nämlich die Nutposition $x_{1\left(\mathrm{~N}_{1}\right)}$, 
derart gewählt, dass der Bereich der geringsten Luftspaltweite groß, also dominant wird und die schmaleren Zähne eine akzeptable Breite behalten.

\subsubsection{Ständer mit originaler Nutschlitzbreite}

Bei Betrachtung von Maschine „C" mit originaler Nutschlitzbreite treten die in Bild 7.19a ersichtlichen signifikanten Einbrüche der Induktion im Bereich der Nuten in Erscheinung. Mit diesen Leitwerteinbrüchen gehen gegenüber der Variante mit schmalen Nutschlitzen deutlich höhere nutharmonische Anteile der Ständerinduktion einher, wie diese im Spektrum für die Variante mit herkömmlichem Blechschnitt in Bild 7.20a erkennbar sind.

Eine Reduktion der nichtnutharmonischen Anteile in der ständerseitig erregten Luftspaltinduktion lässt sich durch Anwendung der Variation der Luftspaltweiten erreichen. Das zugehörige Spektrum ist ebenfalls in Bild 17.20a zu erkennen. Dabei sei bei Vergleich der Spektren der Maschine mit schmalen und der mit breiten Nutschlitzen auf die unterschiedlichen Maßstäbe von Bild 7.18 und Bild 7.20 hingewiesen.

Interessanterweise führt die Anwendung der Phasenwinkelkorrektur bei der Maschine mit der originalen Nutschlitzbreite zwar zu dem in Bild 7.19b dargestellten Induktionsverlauf, dessen Plateaus jeweils exakt mittig auf der Induktionshauptwelle liegen. Das zugehörige Spektrum, welches in Bild 7.20b als „ohne Luftspaltweitenkorrektur" dargestellt ist, weist jedoch viele deutliche Oberwellen auf.

Diese Oberwellen lassen sich durch die nun nicht mehr streng in $N_{1}$ gleichen Perioden am Umfang angeordneten Ständernuten und den damit verbundenen Leitwerteinbrüchen begründen. Zudem tritt hier der von den unterschiedlichen Verhältnissen zwischen Zahnund Nutbreiten abhängige in Abschnitt 6.4.1 erläuterte Einfluss der Phasenwinkelkorrektur auf die Variation der Luftspaltweite auf.

Der letztgenannte Einfluss lässt sich dabei, zumindest für die Luftspaltinduktionswellen mit betragsmäßig niedrigen räumlichen Ordnungszahlen, wie in Abschnitt 6.4.1 vorgeschlagen, durch eine nachgelagerte Luftspaltweitenkorrektur reduzieren.

Diese Korrektur der Luftspaltweiten unter Einbeziehung nicht nur der Zähne selbst, sondern der Luftspaltleitwerte, welche sich zwischen je zwei benachbarten Nutmitten ergeben, führt zu dem in Bild $7.19 \mathrm{~d}$ dargestellten Induktionsverlauf und zu dem in Bild 7.20b für die Variante mit Luftspaltweitenkorrektur dargestellten Induktionsspektrum.

Der Vergleich von Bild 7.19a und Bild 7.19c zeigt dabei, dass die nachgelagerte Luftspaltweitenkorrektur in Bezug auf die Induktionsamplituden an den einzelnen Ständerzähnen aufgrund der Wahl von $x_{1\left(\mathrm{~N}_{1}\right)}$ zu einer Wiederannäherung an den nicht modulierten Ständer führt. Die negativen Einflüsse der Luftspaltweitenkorrektur, wie zum Beispiel der durch das hier gewählte $\tilde{\delta}$ engere minimale geometrische Luftspalt, werden damit reduziert.

Ein Vergleich der Spektren in Bild 7.20b zeigt zudem, dass die nachgelagerte Luftspaltweitenkorrektur zu der gewünschten Reduktion der Induktionsoberwellenamplituden führt wenn auch nur in geringer Ausprägung. Dabei wurde die Luftspaltweite $\tilde{\delta}$ im hier gezeigten Beispiel derart gewählt, dass sich für den modulierten und den unmodulierten Fall gleiche mittlere Luftspaltleitwerte ergeben und die Amplituden der Induktionsoberwellen also direkt sinnvoll verglichen werden können. 


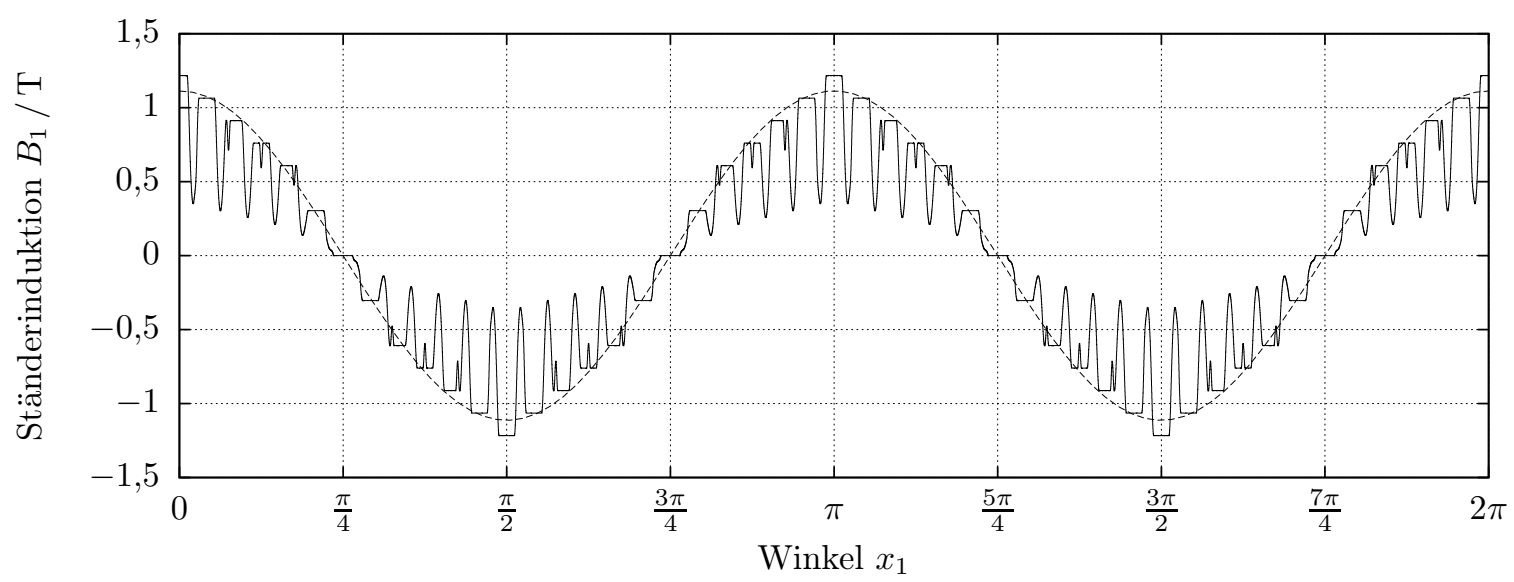

(a) Induktionsverlauf $B_{1}\left(x_{1}, t=0\right)$ bei gleichmäßiger Ständergeometrie

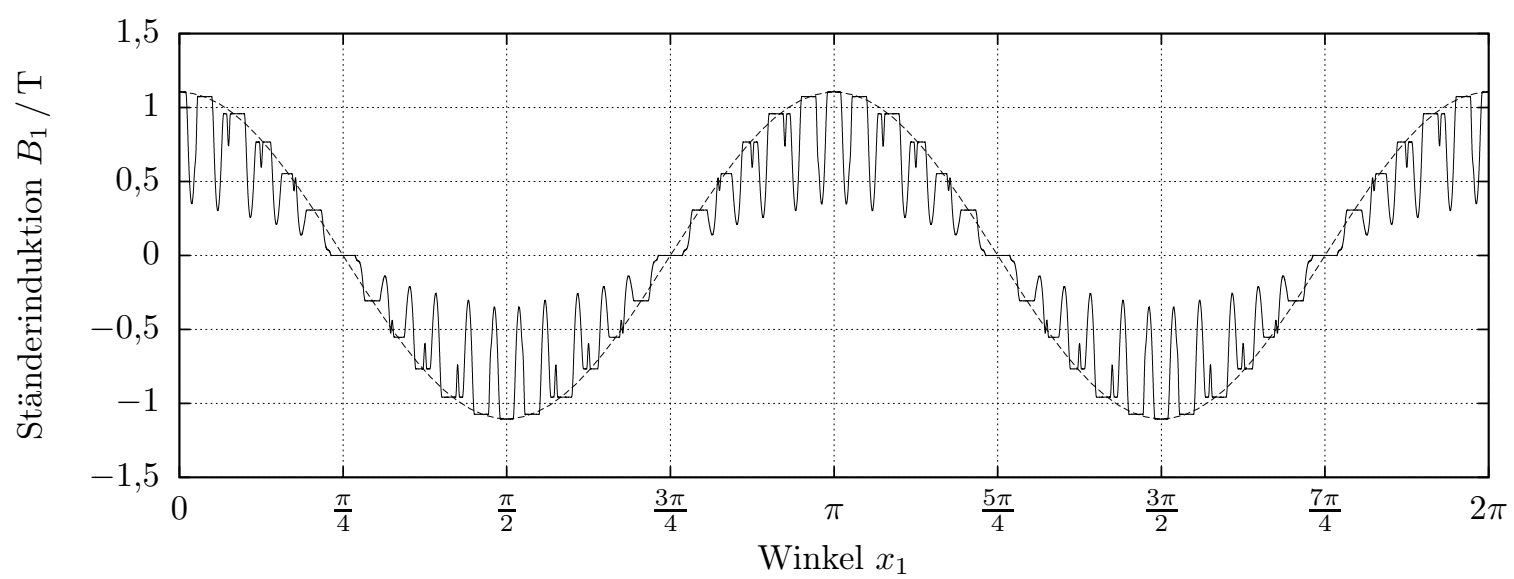

(b) Induktionsverlauf $B_{1}\left(x_{1}, t=0\right)$ bei Luftspaltweitenanpassung und Phasenwinkelkorrektur

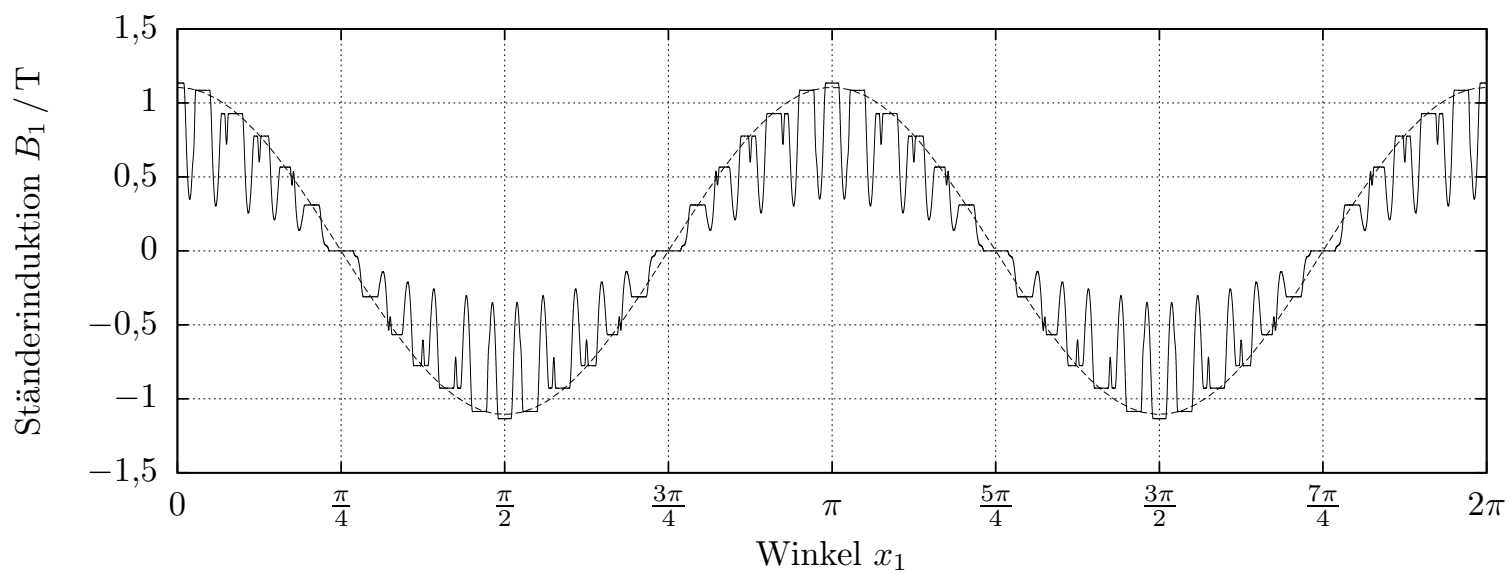

(c) Induktionsverlauf $B_{1}\left(x_{1}, t=0\right)$ bei Luftspaltweitenanpassung und Phasenwinkelkorrektur sowie nutungsbedingter Luftspaltweitenkorrektur

Bild 7.19: Einfluss der Nutpositionen und Luftspaltweiten auf die ständerseitig erregte Induktion von Maschine „C“. - Neben dem resultierenden Induktionsverlauf, in dem der Einfluss der Läufernutung erkennbar ist, sind jeweils die um den Kehrwert des Carter'schen Faktor $1 / k_{\mathrm{c}}$ vergrößerten Induktionshauptwellen dargestellt. 


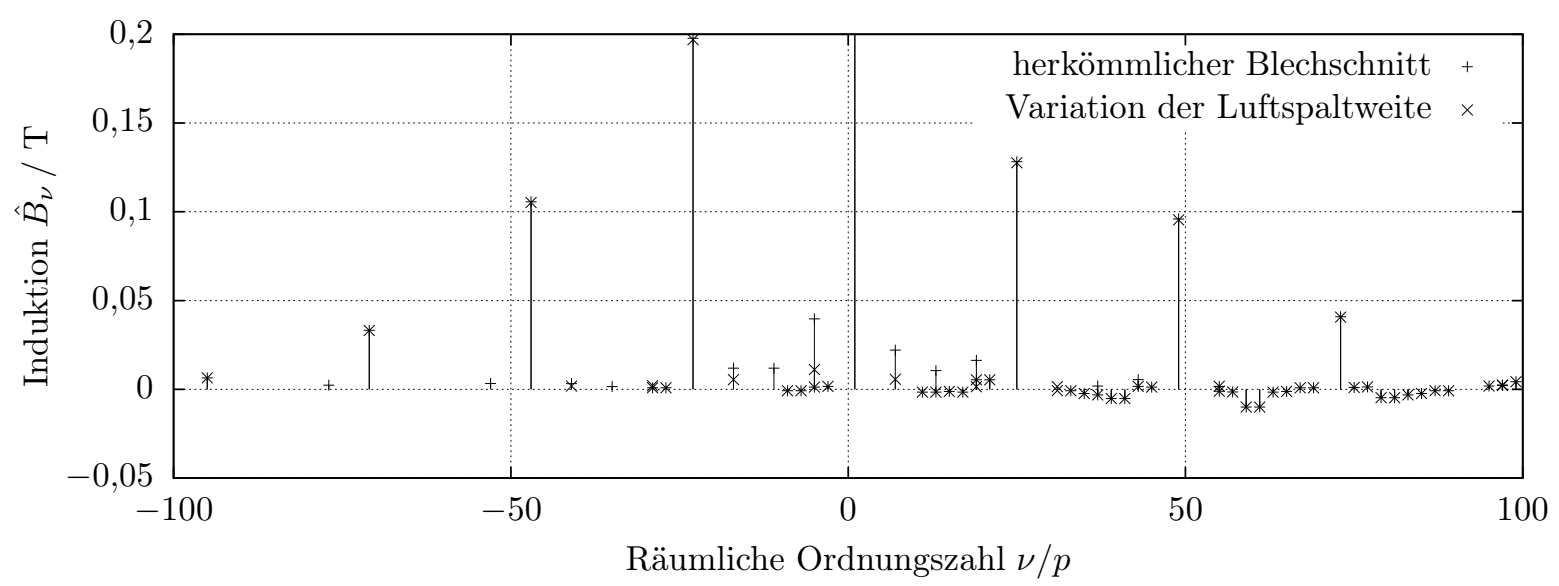

(a) Varianten mit herrkömmlichem Blechschnitt und Variation der Luftspaltweite

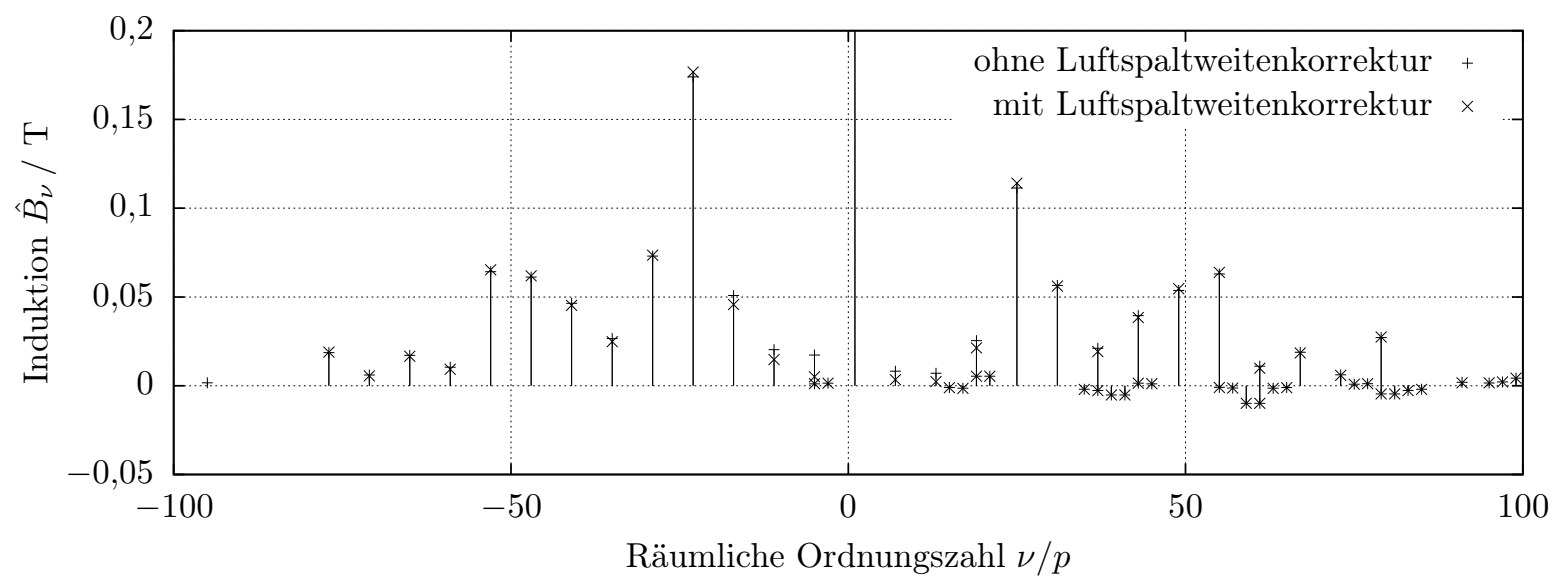

(b) Varianten mit Variation der Luftspaltweite und Phasenwinkelkorrektur sowohl mit als auch ohne Luftspaltweitenkorrektur

Bild 7.20: Spektrum der ständerseitig erregten Luftspaltinduktion von Maschine „C“ 
Einbeziehung einer Läuferrückwirkung Wie in Abschnitt 6.3 beschrieben, beschränkt sich die sinnhafte Anwendung der Luftspaltweitenmodulation auf Maschinen mit geringer Läuferfelderregung. Wird insbesondere die Erregung der räumlichen Ordnungszahl $p$ läuferseitig ähnlich hoch wie ständerseitig, so ergeben sich aus der Läuferfelderregung und den durch die Luftspaltweitenmodulation zusätzlich herbeigeführten Luftspaltleitwertwellen gerade Induktionswellen der Ordnungszahlen, welche durch die Luftspaltweitenmodulation unterdrückt werden sollten. Das Verfahren verfehlt in diesem Fall folglich sein Ziel, nämlich die Unterdrückung aller nichtnutharmonischen Induktionswellen. Damit ist, wie nachfolgend kurz aufgezeigt, mit keiner Verbesserung bezüglich des von der Maschine emittierten Geräusches, sondern vielmehr, wie in Abschnitt 6.4.2 erwähnt, mit zusätzlichen Einzeltönen zu rechnen.

In Bild[7.21a] sind für die Variante mit ausschließlicher Luftspaltweitenmodulation beispielhaft die Schallleistungen der Einzeltöne über der Drehzahl aufgetragen. Ein Vergleich mit Bild $[7.6$ zeigt hier keine wesentlichen Veränderungen gegenüber der Variante ohne Luftspaltweitenmodulation. Dies lässt sich einerseits durch den beschränkten Einfluss der verkleinerten Induktionswellen auf das Geräusch erklären. Andererseits führt die Läuferfelderregung mit den durch die Luftspaltweitenmodulation zusätzlichen Luftspaltleitwertwellen gerade wie eingangs erwähnt zu Induktionswellen derselben räumlichen Ordnungszahlen wie die verkleinerten Induktionswellen.

Für die Variante mit Phasenwinkelkorrektur und nachgelagerter Luftspaltweitenkorrektur ergeben sich die in Bild 7.21b dargestellten Schallleistungen. Hier zeigt sich ein Auftreten weiterer Einzeltöne beziehungsweise das Ansteigen der Schallleistungsamplitude von einzelnen auch bei nicht angewandter ständerseitiger Modulation vorhandenen Komponenten. Erklären lässt sich dies durch die bereits in Bild [7.20 erkennbaren zusätzlichen Ständerinduktionswellen sowie die durch (6.20) beschriebenen zusätzlichen Jochzugwellen aufgrund der nicht mehr streng periodisch verteilten Angriffspunkte der Zahnkräfte am Joch.

Es bestätigt sich damit die in Kapitel 6 getroffene Aussage, dass die vorgeschlagene Luftspaltweitenmodulation in Bezug auf den emittierten Schall für Maschinen mit signifikanter Läuferfelderregung insbesondere bei Anwendung der Phasenwinkelkorrektur nicht zielführend ist.

\subsubsection{Reluktanzmaschine auf Basis von Maschine „C“}

Wie im letzten Abschnitt gezeigt, schwindet die Sinnhaftigkeit der Luftspaltweitenmodulation mit zunehmender Läuferfelderregung. Besonders vorteilhaft ist ihr Einsatz daher bei Reluktanzmaschinen, da diese keine Läuferfelderregung besitzen. Zur Überprüfung dieser Annahme werden die in Bild 7.22 dargestellten Varianten einer auf Maschine „C“ basierenden Reluktanzmaschine untersucht.

Von den beiden Reluktanzmaschinenvarianten sind in Bild [7.23 jeweils die Komponenten der Luftspaltinduktion dargestellt, welche die Frequenz $f_{1}=50 \mathrm{~Hz}$ besitzen. Erwartungsgemäß zeigt sich dabei für die Variante mit Luftspaltweitenmodulation eine Reduktion aller signifikanten und nicht nutharmonischen Induktionswellen. Dies sind im Ausschnitt der Darstellung die Wellen mit den räumlichen Ordnungszahlen $\nu=$ 


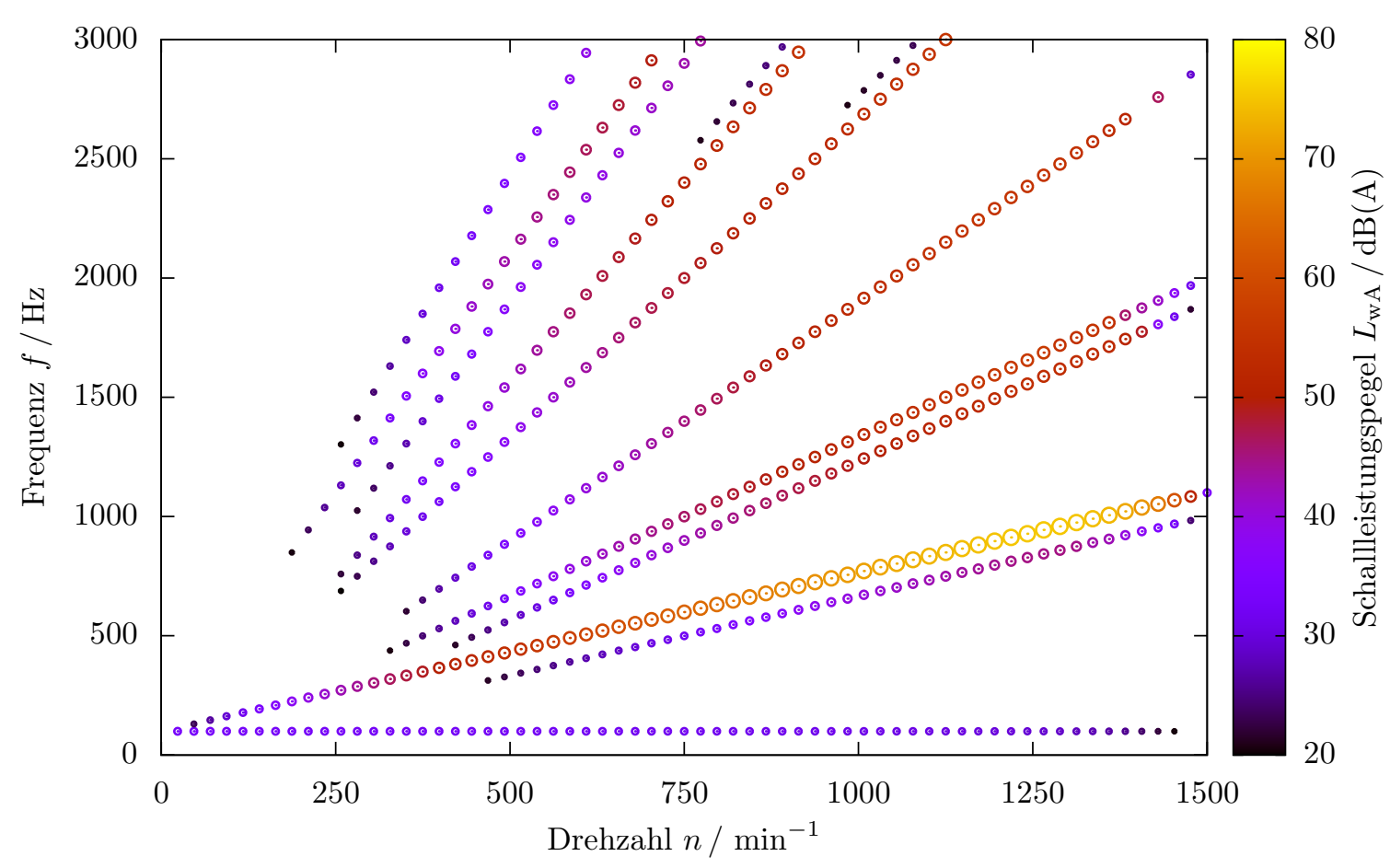

(a) Variante mit Luftspaltweitenmodulation ohne Phasenwinkelkorrektur

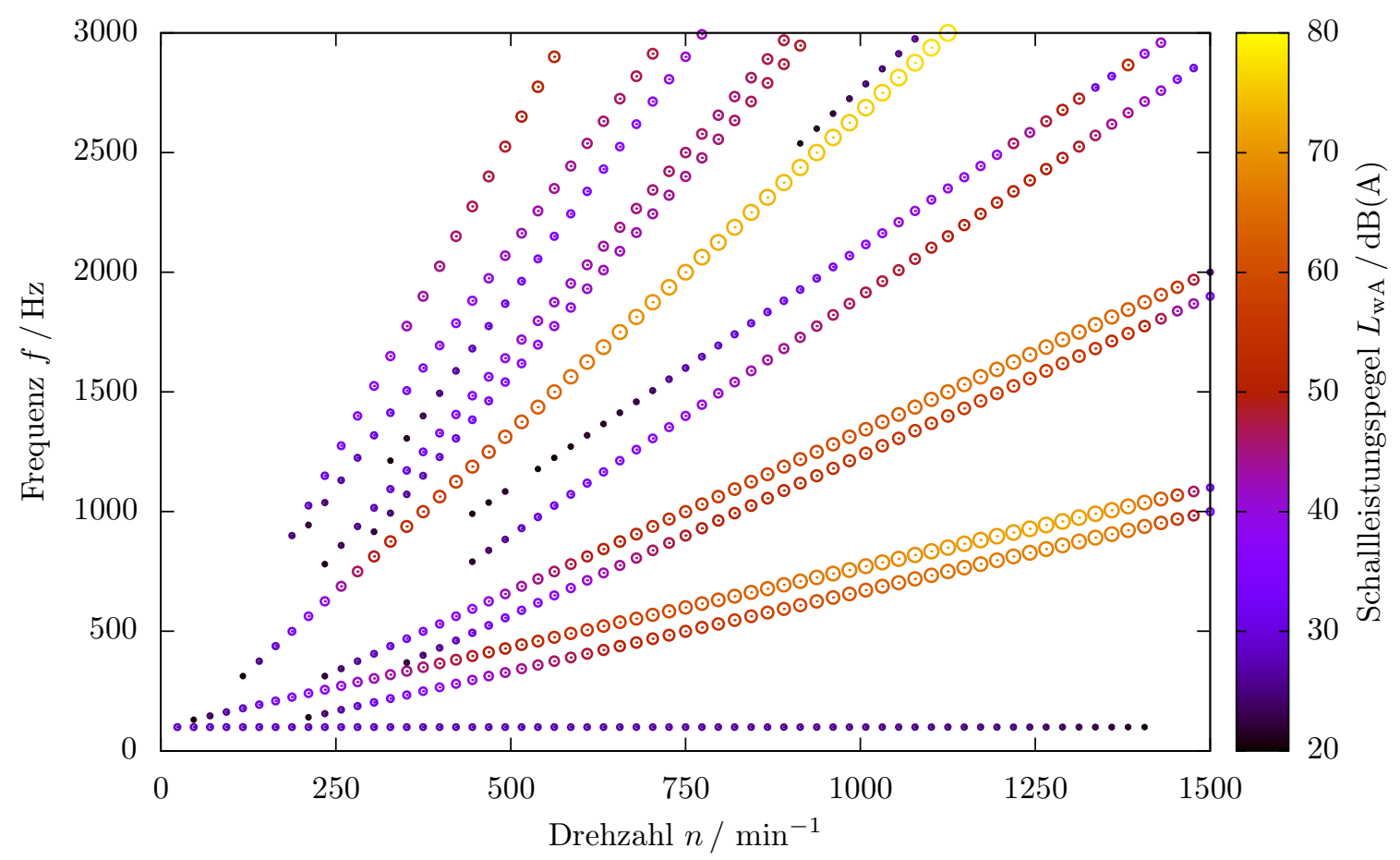

(b) Variante mit Luftspaltweitenmodulation, Phasenwinkelkorrektur und nachgelagerter Luftspaltweitenkorrektur

Bild 7.21: Schallleistung der Einzeltöne von Maschine „C" mit $N_{2}=40$ Läufernuten bei Betrieb an $U_{1, \mathrm{str}}=398 \mathrm{~V}$ mit $f_{1}=50 \mathrm{~Hz}$ über der Drehzahl und Frequenz 


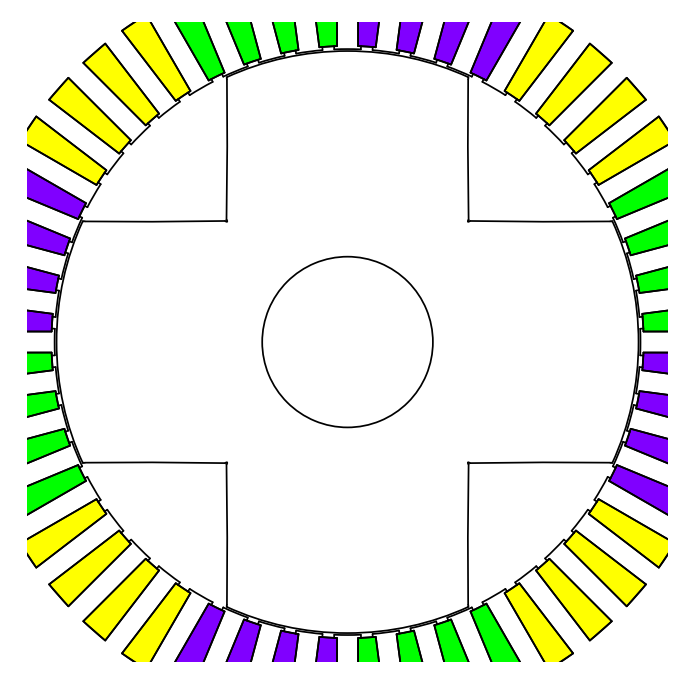

(a) Herkömmlicher Blechschnitt

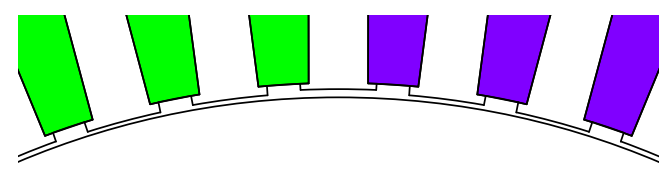

(c) Ständer mit Luftspaltweitenmodulation Ausschnittvergrößerung

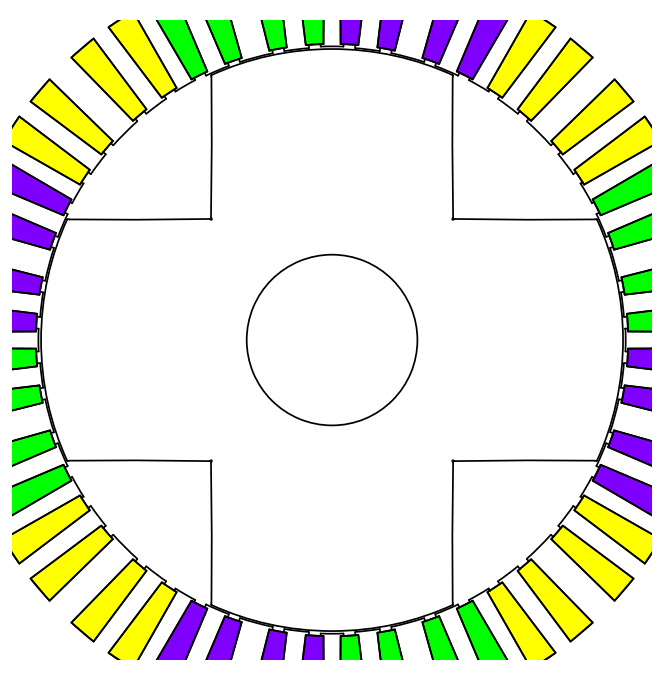

(b) Ständer mit Luftspaltweitenmodulation, Phasenwinkelkorrektur und Luftspaltweitenkorrektur

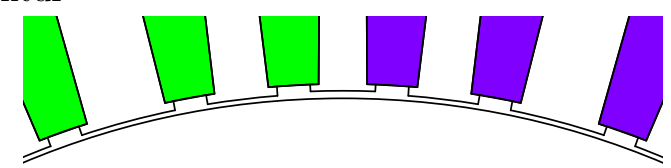

(d) Ständer mit Luftspaltweitenmodulation, Phasenwinkelkorrektur und Luftspaltweitenkorrektur - Ausschnittvergrößerung

Bild 7.22: Reluktanzmaschine auf Basis von Maschine „C“; fünffach überhöhte Darstellung der Luftspaltweitenmodulation

$-5 p, 7 p,-11 p, 13 p,-17 p, 19 p$. Bei gleichzeitiger Phasenwinkel- und Luftspaltweitenkorrektur ergibt sich hingegen zwar eine Reduktion der nutharmonischen Komponenten mit $\nu=-23 p$ und $\nu=25 p$, jedoch auch eine Vergrößerung der Komponenten mit den räumlichen Ordnungszahlen $\nu=-11 p,-17 p, 19 p$. Dieses Ergebnis entspricht weitgehend dem in Abschnitt 7.4.2.2 für die ständerseitig erregte Induktion gezeigten und lässt sich somit auch entsprechend der dortigen Argumentation erklären.

Die Luftspaltweitenmodulation erlaubt somit bei Synchronreluktanzmaschinen eine Verringerung der nichtnutharmonischen Luftspaltinduktionsoberwellenamplituden. Folglich lässt sich mit diesem Verfahren ohne Sehnung der Ständerwicklung läuferseitig eine konstantere Induktion und somit geringere Eisenverluste erreichen. Synchronreluktanzmaschinen stellen daher einen sinnvollen Einsatzbereich der Luftspaltweitenmodulation dar. 


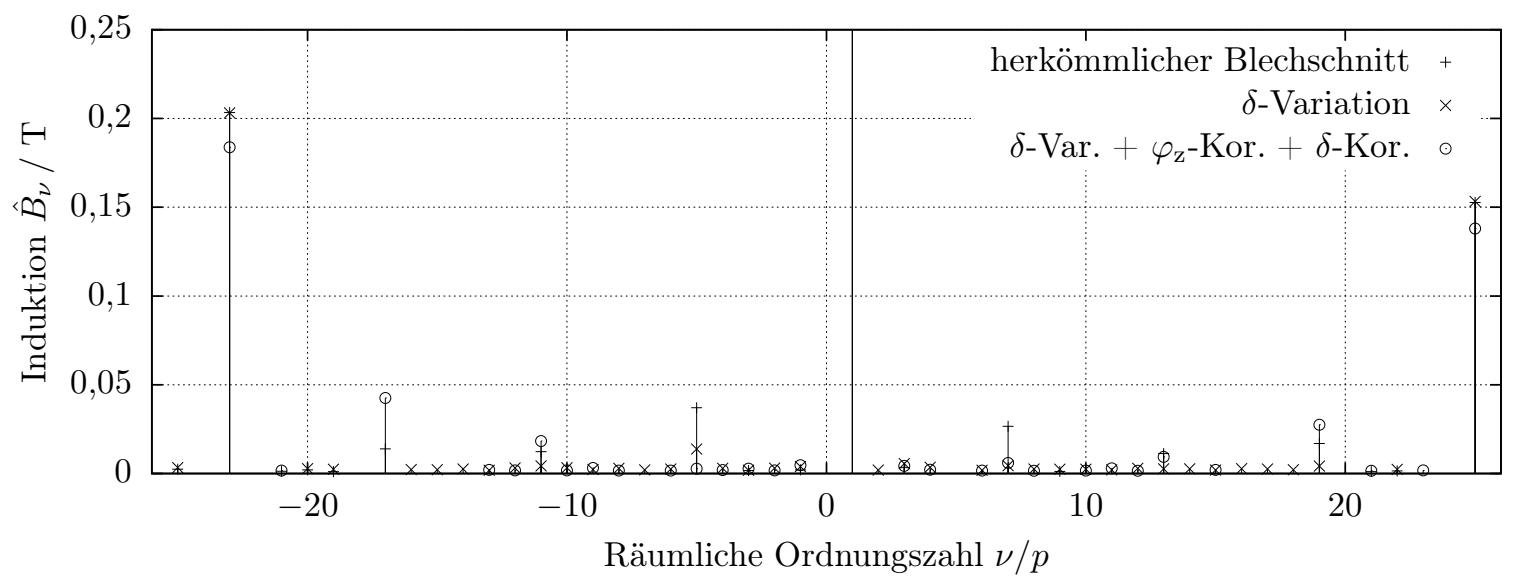

Bild 7.23: Mit Hilfe der Finite-Elemente-Methode berechnetes Spektrum der Luftspaltinduktionswellen der Reluktanzmaschine mit herkömmlichem Blechschnitt, Variation der Luftspaltweite $(\delta$-Variation) sowie Variation der Luftspaltweite und gleichzeitiger Phasenwinkelkorrektur sowie Luftspaltweitenkorrektur $\left(\delta\right.$-Var. $+\varphi_{\mathrm{z}}$-Kor. $+\delta$-Kor. $)$. Es sind ausschließlich Induktionswellen mit $f_{1}=50 \mathrm{~Hz}$ dargestellt. Die Luftspaltweite $\tilde{\delta}$ gemäß (6.13) ist jeweils derart gewählt, dass sich in allen Varianten die gleiche Induktionshauptwellenamplitude ergibt. 


\section{Zusammenfassung}

Ausgehend von der in Kapitel 1 vorgestellten Idee, den Aufbau unregelmäßiger Geometrien von zum Beispiel Ventilatoren und Fahrzeugreifen auf elektrische Maschinen zu übertragen, konnte in den Kapiteln 3 und 4 ein umfangreiches Formelwerk zur Berechnung derartiger Geometrien bei vornehmlich Induktionsmaschinen mit Käfigläufer aufgebaut werden. Auch wenn der Fokus in den genannten Kapiteln primär auf einer Variation der Positionen und Geometrien der Läufernuten liegt, sind die meisten der dort aufgezeigten Zusammenhänge allgemein gültig; also auch für Variationen im Ständer.

Die letztgenannte Art der Variation, nämlich die im Ständer, wurde unter dem Aspekt der Ausbildung der insbesondere ständerseitig erregten Luftspaltinduktionswellen in Kapitel 6 gesondert betrachtet. Dabei wurde ein Verfahren entwickelt, mit dessen Hilfe das Spektrum der genannten Luftspaltinduktionswellen möglichst oberwellenarm gestaltet werden kann.

Die in Kapitel[6] und Abschnitt 7.4 dargestellten Untersuchungen der genannten Variation der Ständergeometrie zeigen dabei, dass sich der Vorteil, mit fertigungstechnisch relativ einfachen Mitteln eine verhältnismäßig oberwellenarme Luftspaltinduktion zu generieren, auf Maschinen mit gegenüber dem Ständer geringer Felderregung des Läufers beschränkt. Das bedeutet, dass die gezeigte Idee vor allem in Reluktanzmaschinen Anwendung finden könnte. Zusätzlich zur genannten Einschränkung ergibt sich, wie in Abschnitt 6.4.2 aufgezeigt, durch die Variation der Ständergeometrie bei Anwendung der Phasenwinkelkorrektur eine veränderte Übertragung zum Beispiel der Radialkraftwellen auf die Jochzugwellen, was sich durch einen Anstieg der subjektiv empfundenen Lautheit im Geräuschspektrum einer elektrischen Maschine widerspiegeln kann.

Bezüglich der Variation der Läufernutpositionen lassen sich bereits im Rahmen der Entwicklung des Formelwerks, aber auch anhand der theoretischen Untersuchungen in Kapitel 5 einige grundlegende Zusammenhänge erkennen. Hierbei sei zum Beispiel darauf verwiesen, dass das Spektrum der magnetisch angeregten Geräusche von elektrischen Maschinen gemäß (4.66) nur aus bestimmten diskreten Einzeltönen bestehen kann. Ein weißes oder rosa Rauschen ist damit nicht erreichbar. Vielmehr beschränken sich die Möglichkeiten der Variation der Geometrie auf die Aufweitung des Spektrums auf eine erhöhte Anzahl diskreter Einzeltöne, welche dabei ähnlich einem weißen oder rosa Rauschen verteilt sein könnten.

Einen weiteren Einblick erlauben die in Kapitel[7 untersuchten Beispiele. Mit den dort gezielt aufgeweiteten Geräuschspektren geht eine Aufweitung der Spektren der räumlichen Ordnungszahlen der Luftspaltinduktionswellen einher. Damit entstehen abhängig von der Drehzahl schnell unerwünschte Wechselwirkungen, welche teilweise praktisch nicht mehr überblickbar sind. Zudem ergeben sich sehr viele Jochzugwellen niedriger räumlicher Ordnungszahl, die aufgrund der höheren relativen Schallleistung potentiell auf laute Einzel- 
töne führen. Aus diesem Grund erscheint eine gewisse Regelmäßigkeit in der Geometrie, wie dies auch Chitroju in seiner Arbeit [5] herausgestellt hat, als vorteilhaft.

Als negativer Effekt aufgrund von variierten Läufernutpositionen kann beispielhaft, neben der Anregung weiterer dominanter Einzeltöne mit bereits bei regelmäßiger Geometrie angeregten Schwingungsformen, die Anregung von Schwingungen mit gegenüber der regelmäßigen Geometrie niedrigeren räumlichen Ordnungszahlen genannt werden. In diesem am Beispiel der Modulation von Maschine „C" mit 10 Blöcken in Abschnitt [7.3.4.1] gezeigten Fall ergeben sich höhere relative Schallleistungen und damit schnell signifikante Einzeltöne.

Es soll zudem auf die anhand des Beispiels in Abschnitt 7.3.4.1 bei Modulation in 4 Blöcken dargestellte Möglichkeit des Auftretens von Schwebungen im Geräusch hingewiesen werden. Aufgrund des leicht entstehenden Eindrucks, eine Maschine mit einer deutlichen Schwebung im Geräusch könnte eine langsame Drehmomentpendelung aufweisen, werden derartige Maschinen möglicherweise kundenseitig negativ bewertet.

Letztendlich zeigt sich für die Variation der Läufernutpositionen und -formen, dass das Geräuschspektrum einiger Maschinen unter Berücksichtigung weiterer Parameter, wie zum Beispiel dem Drehmoment inklusive seiner Pendelungen, gemäß einer objektiven Bewertung deutlich angenehmer gestaltbar ist. Für die meisten Geometrien ist jedoch kein merklich angenehmeres Spektrum erzielbar. Bezüglich der Luftspaltweitenmodulation lässt sich kein positiver Effekt im Sinne eines angenehmeren Geräuschspektrums feststellen. Dennoch ist das Verfahren bei Maschinen mit geringer läuferseitiger Felderregung, also insbesondere bei Synchronreluktanzmaschinen, zur Reduktion vieler Induktionsoberwellenamplituden sinnvoll anwendbar.

Zudem ließen sich keine Regeln finden, wie insbesondere die Läufernutpositionen zur Reduktion der Lautheit einer Maschine exakt zu wählen sind. Dieses wäre dabei wünschenswert gewesen, war jedoch auch nicht zu erwarten, da die in Kapitel11 genannten Veröffentlichungen bezügliche der Ausführung von Variationen bei Fahrzeugreifen und Ventilatoren für diese Produktgruppen ebenfalls keine derartigen Regeln zeigen.

Es drängt sich daher als Weiterarbeit eine systematische numerische, aber auch messtechnische Untersuchung von durch analytische Berechnung optimierten Geometrien auf, womit sich eventuell technisch und wirtschaftlich sinnvolle Geometrien mit variierten Nutpositionen und -formen finden ließen. 


\section{Literaturverzeichnis}

[1] Boltezar, M. ; Mesaric, M. ; Kuhelu, A. : The Influence of Uneven Blade Spacing on the SPL and Noise Spectra Radiated From Radial Fans. In: Journal of Sound and Vibration (1998), S. 697-711

[2] Braun, M. ; Vollmer, R. : Drehstromasynchronmaschine. Patent: DE 102004010 386 A1, 2005

[3] Carter, F. W.: Air-Gap Induction. In: Electrical World and Engineering 29 (1900), Nr. 146, S. $884-888$

[4] Cattanei, A. ; Ghio, R. ; Bongiovì, A. : Reduction of the tonal noise annoyance of axial flow fans by means of optimal blade spacing. In: Applied Acoustics 68 (2007), S. $1323-1345$

[5] Chitroju, R. K. S.: Improved Performance Characteristics of Induction Machines with Non-Skewed Asymmetrical Rotor Slots, School of Electrical Engineering, Royal Institute of Technology (KTH), Stockholm, Diss., 2009

[6] Dobrzynski, W. : Propeller Noise Reduction by Means of Unsymmetrical BladeSpacing. In: Journal of Sound and Vibration (1993), Nr. 163, S. 123-136

[7] Edrei, A. ; Szegö, G. : A Note on the Reciprocal of a Fourier Series. In: Proceedings of the American Mathematical Society 4 (1953), May, Nr. 2, S. 323-329

[8] Ewald, D. ; Pavlovic, A. ; Bollinger, J. G.: Noise Reduction by Applying Modulation Principles. In: The Journal of the Acoustical Society of America (1970), November, S. 1381-1385

[9] Henn, H. ; Sinambari, R. ; Fallen, M. : Ingenieurakustik. 4. Wiesbaden : Vieweg+Teubner, 2008. - ISBN 978-3-8348-0255-2

[10] HumburG, K. : Die Entstehung des Drehmomentes in elektrischen Maschinen. In: Elektrotechnische Zeitschrift 71. Jahrgang (1950), Juni, Nr. 12, S. 311-313

[11] Jordan, H. : Der geräuscharme Elektromotor. Essen : Verlag W. Giradet, 1950

[12] Jordan, H. ; LAX, F. : Untersuchung des Einflusses einer nicht in den Nutmittellinien konzentriert vorausgesetzten Durchflutung auf die doppeltverkettete Streuung. In: Elektrotechnik und Maschinenbau (1940), S. 393-396

[13] Jordan, H. ; Weis, M. : Nutenschrägung und ihre Wirkungen. In: ETZ-A Bd 88 (1967), S. 528-533

[14] KolBe, J. : Zur numerischen Berechnung und analytischen Nachbildung des Luftspaltfeldes von Drehstrommaschinen, Hochschule der Bundeswehr Hamburg, Diss., 1983

[15] Küpfmüller, K. ; Mathis, W. ; Reibiger, A. : Theoretische Elektrotechnik. 17. Springer, 2006. - ISBN 3-540-29290-X 
[16] Loskutov, A. : Generierung und heuristische Optimierung von Reifenprofilen, Institut für Systems Engineering, Universität Hannover, Diss., 2005

[17] Morimoto, M. ; Morita, I. : Characteristic Analysis of an Induction Motor with an Unequal Slot Pitch Cage Rotor. In: Elektrical Engineering in Japan 140 (2002), Nr. 2, S. 44-52

[18] MÜller, G. ; POnICK, B. : Elektrische Maschinen. Bd. 3: Theorie elektrischer Maschinen. 6. Berlin: Wiley-VCH, 2009. - ISBN 3-527-40526-7

[19] Müller, G. ; Vogt, K. ; Ponick, B. : Elektrische Maschinen. Bd. 2: Berechnung elektrischer Maschinen. 6. Berlin: Wiley-VCH, 2007. - ISBN 3-527-40525-9

[20] MÜller-TOMfelde, H. : Einfluss der Schrägung auf den magnetischen Lärm, die Erwärmung und die Pendelmomente, Fakultät für Maschinenwesen der Technischen Hochschule Hannover, Diss., 1962

[21] Nürnberg, W. : Die Asynchronmaschine. 2. Berlin : Springer, 1963

[22] OHм, J.-R. ; LÜKE, H. D.: Signalübertragung: Grundlagen der digitalen und analogen Nachrichtenübertragungssysteme. 11. Berlin : Springer, 2010. - ISBN 978-3642101991

[23] Ponick, B. : Fortschritts-Berichte VDI Reihe 21, Nr.. Bd. 174: Fehlerdiagnose bei Synchronmaschinen. Düsseldorf: VDI Verlag, 1995. - ISBN 3-18-317421-9

[24] Ponick, B. : Das Luftspaltmoment elektrischer Maschinen unter Berücksichtigung parametrischer Effekte. In: Electrical Engineering 81 (1998), S. 291-296

[25] Prokott, E. : Modulation und Demodulation. AEG-Telefunken, 1977

[26] RAsche, C. : Berechnung des Feldspektrums einer Induktionsmaschine mit Käfigläufer unter Berücksichtigung der Eisensättigung, Diplomarbeit 506, Institut für elektrische Maschinen und Antriebe - Universität Hannover, Diplomarbeit, 1989

[27] RÅde, L. ; Westgren, B. : Mathematics Handbook. Berlin, Heidelberg, New York: Springer, 2004. - ISBN 3-540-21141-1

[28] Seinsch, H. O.: Oberfelderscheinungen in Drehfeldmaschinen. B. G. Teubner, 1992. - ISBN 3-519-06137-6

[29] Strauss, T. : Berechnung des Geräuschpegels von Drehfeldmaschinen, Institut für elektrische Maschinen und Antriebe, Universität Hannover, Diss., 1987

[30] Taegen, F. ; WalczaK, R. : Eine experimentell überprüfte Vorausberechnung der Oberfelder von Käfigläufermotoren. In: Archiv für Elektrotechnik 66 (1983), S. 233242

[31] Tzscheutschler, R. ; Olbirsch, H. ; Jordan, W. : Technologie des Elektromaschinenbaus. 1. Berlin : Verlag Technik GmbH Berlin, 1990. - ISBN 3-341-00851-9

[32] Wachta, B. : Influence of Stator Slots on the Development of Noise-Generating Magnetic Force Waves. In: Siemens Forschungs- und Entwicklungsberichte 10 (1981), Nr. 5, S. 289-298

[33] WEH, H. : Zur elektromagnetischen Schwingungsanregung bei Asynchronmaschinen. In: Elektrotechnische Zeitschrift 7 (1964), April, S. 193-197

[34] WieBICKe, G. : Fortschritts-Berichte VDI Reihe 21, Nr.. Bd. 368: Beitrag zur Lastabhängigkeit des Luftspaltfeldes der Polpaarzahl 3p bei Käfigläufern. Düsseldorf: VDI Verlag, 2005. - ISBN 3-18-336821-8 
[35] Wolff, I. : Maxwellsche Theorie. 4. Berlin : Springer, 1997. - ISBN 3-540-63012-0 


\section{Wissenschaftlicher Werdegang}

\section{Persönliche Daten}

Name:

Geburtsdatum:

Geburtsort:

\section{Studium}

Okt. 2003 - Mrz. 2008 Studium der Elektrotechnik an der Leibniz Universität Hannover, Studienrichtung Automatisierungstechnik, Abschluss: Diplomingenieur

seit Apr. 2008

\section{Beruf}

Apr. 2008 - Mrz. 2011

Wissenschaftlicher Mitarbeiter am Institut für Antriebssysteme und Leistungselektronik (IAL) der Leibniz Universität Hannover

Apr. 2011 - Aug. 2014 Entwicklungsingenieur bei der Wittenstein cyber motor GmbH, Igersheim

seit Sep. 2014
Eike Matthias Garbe

12.11.1982

Hannover trotechnik und Informatik der Leibniz Universität Hannover

Høgskolelektor (assistant professor), Høgskolen i Ålesund, Norwegen 